\title{
Elucidating the underlying mechanisms of inflammation caused by perinatal asphyxia and chorioamnionitis
}

Citation for published version (APA):

Vlassaks, E. (2012). Elucidating the underlying mechanisms of inflammation caused by perinatal asphyxia and chorioamnionitis. [Doctoral Thesis, Maastricht University]. Uitgeverij BOXPress.

https://doi.org/10.26481/dis.20121217ev

Document status and date:

Published: 01/01/2012

DOI:

10.26481/dis.20121217ev

Document Version:

Publisher's PDF, also known as Version of record

Please check the document version of this publication:

- A submitted manuscript is the version of the article upon submission and before peer-review. There can be important differences between the submitted version and the official published version of record.

People interested in the research are advised to contact the author for the final version of the publication, or visit the DOI to the publisher's website.

- The final author version and the galley proof are versions of the publication after peer review.

- The final published version features the final layout of the paper including the volume, issue and page numbers.

Link to publication

\footnotetext{
General rights rights.

- You may freely distribute the URL identifying the publication in the public portal. please follow below link for the End User Agreement:

www.umlib.nl/taverne-license

Take down policy

If you believe that this document breaches copyright please contact us at:

repository@maastrichtuniversity.nl

providing details and we will investigate your claim.
}

Copyright and moral rights for the publications made accessible in the public portal are retained by the authors and/or other copyright owners and it is a condition of accessing publications that users recognise and abide by the legal requirements associated with these

- Users may download and print one copy of any publication from the public portal for the purpose of private study or research.

- You may not further distribute the material or use it for any profit-making activity or commercial gain

If the publication is distributed under the terms of Article $25 \mathrm{fa}$ of the Dutch Copyright Act, indicated by the "Taverne" license above, 


\section{Elucidating the underlying mechanisms of inflammation caused by perinatal asphyxia and chorioamnionitis}

Evi Vlassaks 
Copyright: (C) Evi Vlassaks, Maastricht 2012 ISBN: 978-90-8891515-4

Elucidating the underlying mechanisms of inflammation caused by perinatal asphyxia and chorioamnionitis

All rights reserved. No part of this book may be reproduced in any form or by any electronic means, including information, storage and retrieval systems, without permission in writing from the copyright holder.

Cover design by: Ine Vlassaks

Typesetting \& Lay Out by: Evi Vlassaks and Steven Houben

Printed by: Proefschriftmaken.nl II Uitgeverij BOXPress

Published by: Uitgeverij BOXPress, 's-Hertogenbosch 


\title{
Elucidating the underlying mechanisms of inflammation caused by perinatal asphyxia and chorioamnionitis
}

\author{
DISSERTATION \\ To obtain the degree of Doctor \\ at Maastricht University, \\ on authority of the Rector Magnificus, \\ Prof. Dr. L.L.G. Soete,
}

in accordance with the decision of the Board of Deans,

to be defended in public

on Monday 17th of December 2012 at 10.00 o'clock

by

Evi Vlassaks

Born the 15th of October 1986 in Genk (Belgium) 


\section{Promotores:}

Prof. dr. Johan S.H. Vles

Prof. dr. Marc H. de Baets

Prof. dr. Boris W. Kramer

\section{Copromotor:}

Dr. Antonio W.D. Gavilanes

\section{Assessment Committee:}

Prof. dr. L.J.I. Zimmermann (Chair)

Prof. dr. B. Pakkenberg, Bispebjerg Hospital, Copenhagen

Prof. dr. F. Ramaekers

Prof. dr. R.J. van Oostenbrugge

Dr. R.J. Vermeulen, VUMC, Amsterdam 
Chapter 2 Fetal asphyctic preconditioning modulates the acute cytokine response thereby protecting against perinatal asphyxia in neonatal rats

Chapter 3 Fetal asphyxia induces changes in the ceramide pathway and in brain immunological responses

Chapter 4 The effects of fetal and perinatal asphyxia on neuronal cytokine levels and ceramide metabolism in adulthood

Chapter 5 Inflammatory changes and ceramide profiles in rat liver after fetal asphyctic preconditioning and perinatal asphyxia

Chapter 6 Chorioamnionitis induced hepatic inflammation and disturbed lipid metabolism in fetal sheep

Chapter 7 Antenatal exposure to chorioamnionitis affects lipid metabolism in 7-week-old sheep

Chapter 8 Summary and General Discussion

Bibliography

Samenvatting

Abbreviations

Acknowledgements/Dankwoord

Curriculum vitae 



\section{General Introduction}


Perinatal asphyxia (hypoxia-ischemia) and chorioamnionitis are the two major causes of child morbidity and mortality worldwide [1,2]. Although both perinatal insults are known to be associated with inflammation, the exact underlying molecular mechanisms and associated pathology are not clear. Therefore, it is crucial to understand the mediating role of inflammation in the neurological and systemic responses that occur after perinatal asphyxia and chorioamnionitis [3-5].

\section{PERINATAL ASPHYXIA}

Brain injury caused by perinatal asphyxia (PA) is the predominant form of all brain injury encountered in the perinatal period [6]. Globally, between four and nine million infants suffer from PA, accounting for 0.56-0.99 million deaths and approximately the same number of infants who develop severe disability each year [1,7].

PA is a condition of impaired blood gas exchange between mother and fetus during the process of birth, leading to progressive hypoxaemia, hypercapnia and less blood flow with significant metabolic acidosis $[8,9]$. The most common causes responsible for PA are: 1) umbilical cord prolapse, 2) placental abruption, 3) inadequate placental perfusion for instance because of maternal hypotension, and 4) failure of lung expansion or maladaptation of the pulmonary circulation at birth [10].

\section{Consequences and outcomes}

The final outcome of an asphyctic insult significantly varies among the infants, depending not only on the severity and the duration of the insult, but also on the physiological and maturational status of the neonate [11]. Approximately 15-20\% of PA infants die in the neonatal period. Those who survive may develop multiorgan failure, with involvement of many vital organs such as brain, heart, kidneys and liver [12]. When brain injury occurs, a clinically defined syndrome of disturbed neurological function, called hypoxic-ischemic encephalopathy (HIE), takes place within the first hours of life $[8,13,14]$. Infants with mild HIE appear to have a normal neurocognitive outcome, whereas those with moderate to severe HIE will either die or are at high risk to develop cerebral palsy, epilepsy and cognitive, behavioral and memory problems $[9,15]$. 
Since the pattern of brain injury may relate to long-term outcome, accurate neonatal clinical assessment techniques such as neuroimaging, in particular MRI, and neurophysiological tests, like electroencephalogram (EEG), are crucial [16].

\section{Pathophysiology}

Perinatal brain injury is a complex process that is initiated during the asphyctic insult and extends into a recovery period [1]. During the primary phase, energy supplies (mainly adenosine triphosphate -ATP) in the cell are depleted due to oxygen and glucose deprivation. Consequently, functioning of the sodium/potassium ATPase pump is disrupted, thereby inducing depolarisation of the cell membrane and facilitating cytosolic accumulation of calcium $[1,17]$. Additionally, the depolarisation of the cell membrane leads to impaired glutamate uptake and thus accumulation of glutamate in the synaptic cleft. This leads to overactivation of glutamate receptors like the N-methyl-D-aspartic acid (NMDA) receptors in the postsynaptic membrane, inducing even higher calcium influx into the cell [17]. This intracellular accumulation of calcium induces production of nitric oxide (NO) by activation of NO synthase (NOS) [1]. Augmentation of cytosolic calcium levels also results in activation of phospholipases, proteases and endonucleases and leads consequently to the release of free fatty acids, the destruction of proteins and the generation of free radicals. Free radical formation contributes to cell death by energy depletion, mitochondrial impairment and lipid peroxidation $[6,17]$. This cascade, acutely following an asphyctic insult, has as final outcome the degradation of cell integrity and ultimately cell death [18] (Figure 1A).

During the so called reperfusion phase, cerebral circulation and oxygenation are restored. Although cell metabolism partly resolves, injury may further evolve because of additional formation of inflammation and free radicals [18].

The reperfusion phase is followed by the latent phase. During this phase, the concentration of metabolites and intracellular $\mathrm{pH}$ normalizes with transient improvement of cytotoxic edema [1].

Finally, a secondary and delayed phase of brain injury occurs 6 to 15 hours after the initial insult [18], during which neurons and oligodendrocytes continue to 
A

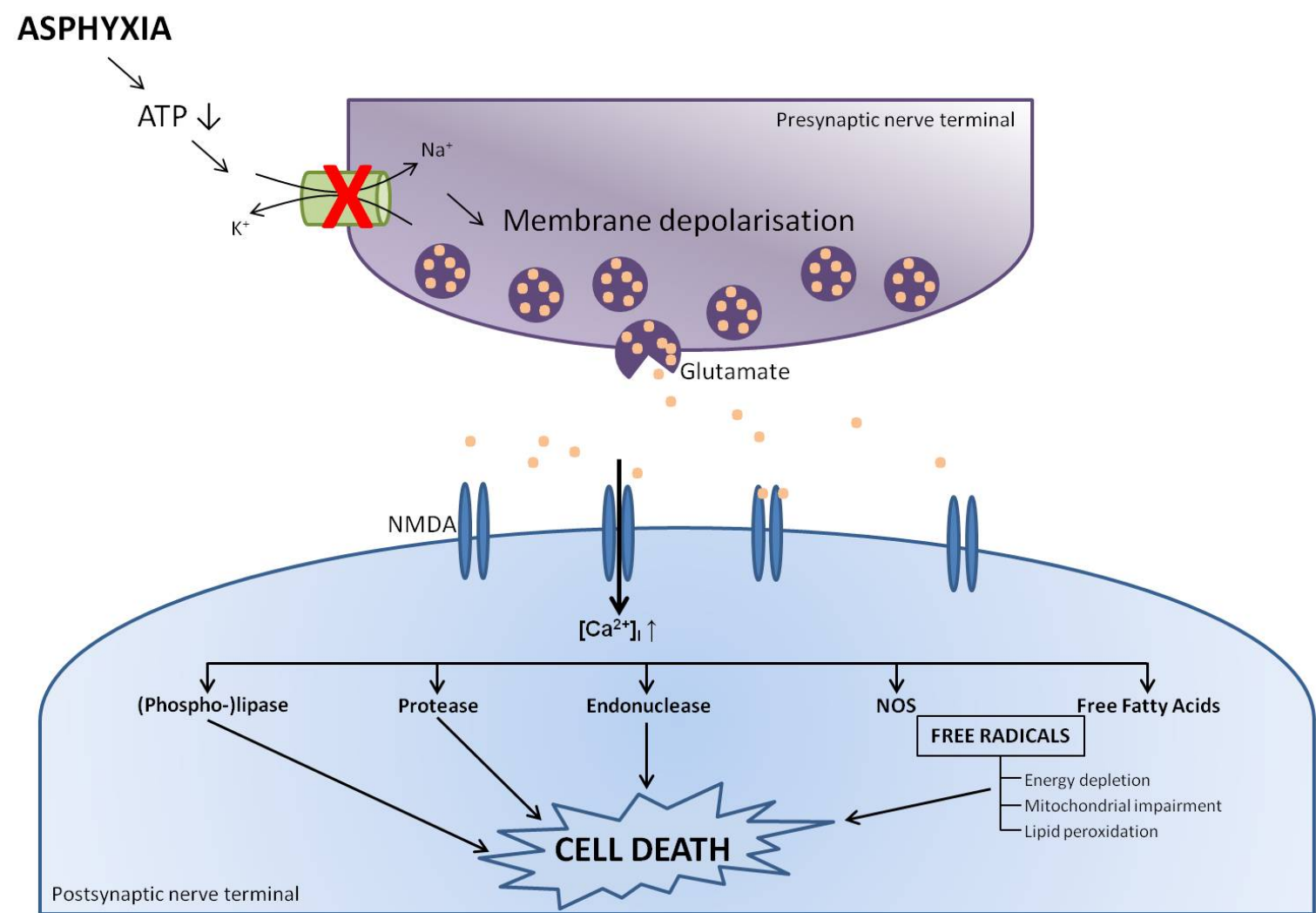

B

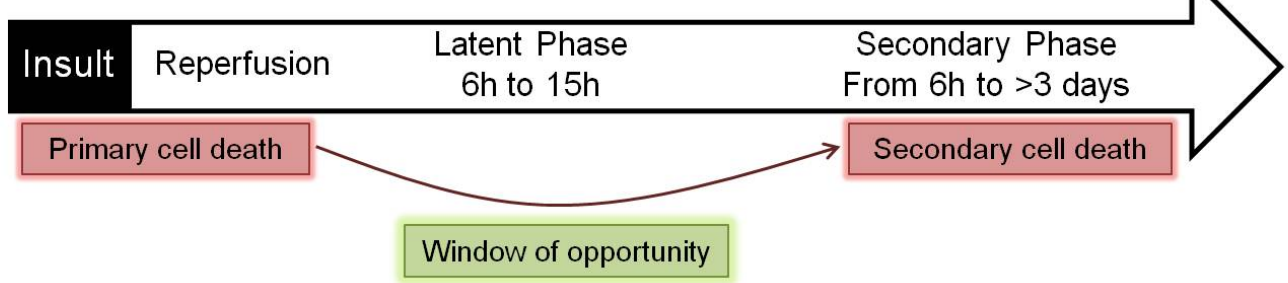

Figure 1: Overview of the pathophysiological mechanisms (A) and scheme of the phases of injury evolving after moderate and severe asphyxia (B) $[1,17,18]$. Asphyxia-induced ATP depletion leads to disruption of the sodium/potassium ATPase pump, thereby inducing depolarisation of the cell membrane. As a consequence, glutamate accumulates in the synaptic cleft leading to intracellular calcium augmentation and ultimately cell death $[1,17]$. 
die for longer periods. The mechanisms involved are similar to those occuring in the primary phase of injury and may be caused because of mitochondrial dysfunction or secondary to extended reactions from the primary insult, like calcium influx, excitatory neurotoxicity or oxygen free radicals. Furthermore, circulatory and endogenous inflammatory cells or inflammatory mediators may contribute to the ongoing brain injury. Cell injury triggers an inflammatory response characterized by the activation of microglia and the infiltration of neutrophils and monocytes, subsequently leading to the production of cytokines and chemokines [1]. Pro-inflammatory cytokines, such as TNF- $\alpha$ and IL-1, may have a direct toxic effect via increased free radical release and indirectly via the induction of microglia and astroglia to produce neurotoxic factors like glutamate $[9,19]$. It is assumed that the secondary phase is a consequence of the cascade of these events rather than a result of cellular destruction. The severity of this secondary energy failure is associated with adverse neurological outcome [1]. An overview of the pathophysiology of perinatal brain injury with corresponding phases is depicted in Figure 1.

\section{Treatment options}

Asphyctic brain injury provides a time window of opportunity for neuroprotective treatment interventions during the latent phase (Figure 1B) [18]. Advances in neuroimaging, brain monitoring techniques and tissue biomarkers (e.g. lactate) have improved the ability to diagnose, monitor and care for newborn infants born with HIE as well as to predict their outcome. Challenges remain in early identification, determination of timing and extent of asphyctic brain injury and optimal treatment strategies $[1,16]$.

The treatment of neonates with asphyctic brain damage was mainly supportive, including blood pressure control, preventing hypotension and hypoglycemia, maintenance of adequate ventilation and treating the possible seizures [9]. Nowadays, therapeutic hypothermia is the standard care for term asphyxiated infants with moderate to severe HIE [1].

Two methods of therapeutic hypothermia are currently available: whole body cooling and selective head cooling with mild systemic hypothermia. Therapeu- 
tic hypothermia aims to lower the temperature of vulnerable brain structures to $\pm 33.5^{\circ} \mathrm{C}$. Hypothermia reduces cerebral metabolism, energy use and NO production. Moreover, it suppresses free radical activity, inflammation and apoptosis; consequently leading to reduced brain injury $[9,18]$. Nevertheless, only 1 in 6 babies suffering from HIE will benefit from hypothermia with the most severe cases still suffering from lifelong permanent disability [1]. Moreover, hypothermia is only applied in term babies implying that no therapy exists for affected preterm babies [11].

\section{Hypoxic-ischemic preconditioning}

During the last decades, the concept of brain preconditioning (Figure 2) has gained a lot of interest. In models of focal asphyxia, it has been shown that sublethal noxious stimuli induce tissue tolerance to a following more severe insult that would otherwise be lethal [20]. The extent of tolerance is dependent on stress sensors, signal transduction pathways and effectors of protection. A stressful condition is detected and converted as an intracellular response. Stimuli like glutamate, proinflammatory cytokines and reactive oxygen species can be recognized by receptors such as neurotransmitter, cytokine and toll-like receptors [21]. Thereafter, processing of the signal occurs through the corresponding signal transduction system and leads to activation of various effector systems that will reduce the vulnerability of the cell or tissue to a new stimulus [20]. Effectors involved in the preconditioning phenomenon are probably related to the cell cycle, metabolism, inflammation and excitotoxicity [21].

In the brain, two forms of preconditioning can be observed: 1) a rapid form which triggers protection within minutes, and 2) a delayed form in which the protected state develops over hours and days [21,22]. The rapid form, which is only short-lasting, is the result of changes in ion channel permeability, protein phosphorylation and other post-translational modifications. The delayed form can provide longer protection and is mediated by gene activation of several families of prosurvival genes and subsequent de novo protein synthesis [21,22]. 


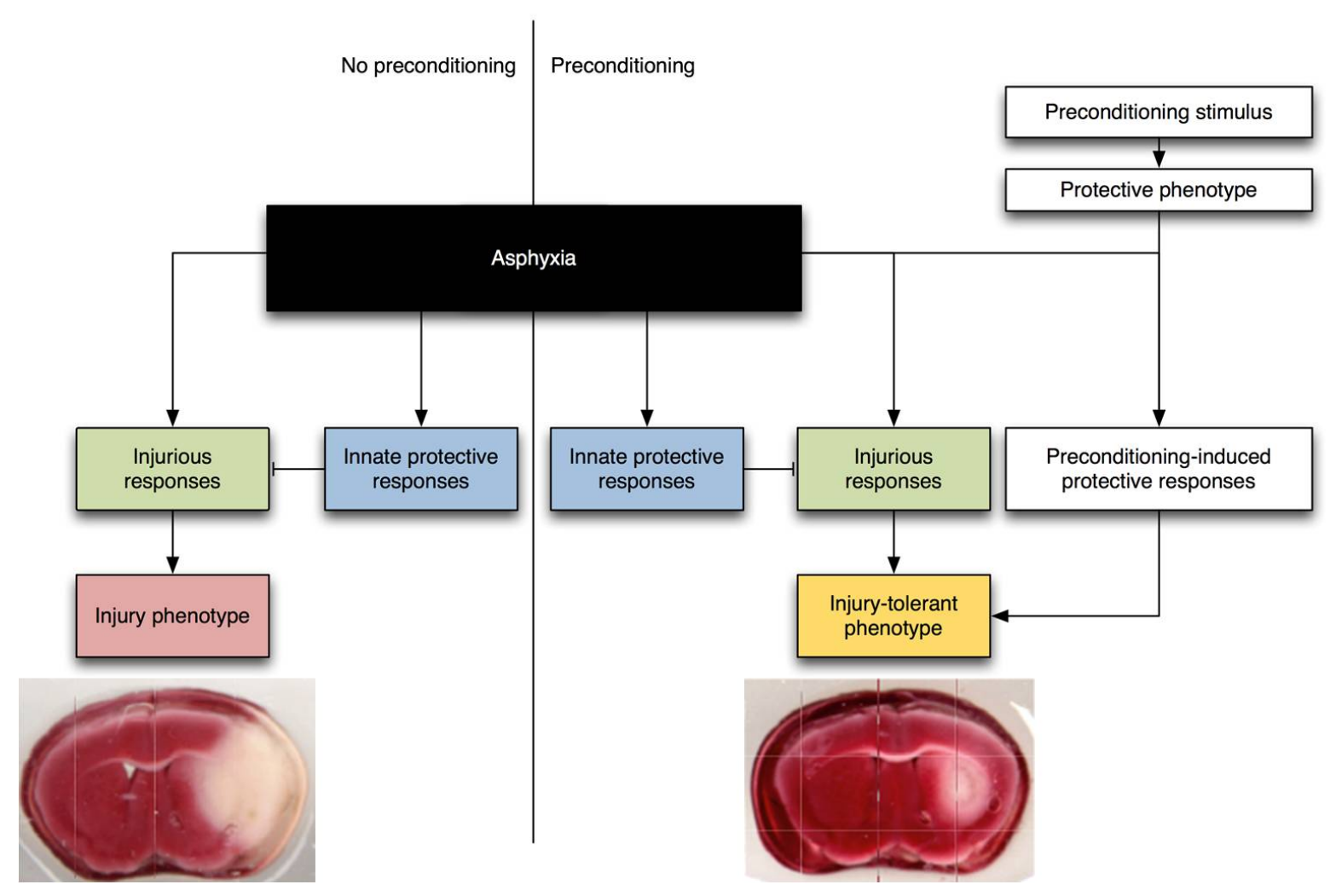

Figure 2: Schematic overview of hypoxic-ischemic preconditioning-induced neuroprotection (Adapted from Gidday et al., 2006). Depicted are brain slices of rats that underwent carotid artery occlusion (CAO) at postnatal day 7 (P7) (left) and ischemic preconditioning at P6 followed by $\mathrm{CAO}$ at P7 (right). Preconditioned animals show a substantial reduction in the severity of brain damage (white area) compared to non-preconditioned animals [22].

Many experimental models are being used, involving different preconditioning stimuli [23-26]. A major limitation in these studies is that almost all animal models use a focal insult, causing one-sided focal brain damage which does not completely mimic the most common pathological type of global HIE and multi-organ failure in the human neonate. Accordingly, we developed a new rat model for fetal asphyctic preconditioning and perinatal asphyxia, inducing two global asphyctic insults. By using this model, we have shown that mild fetal asphyxia as preconditioning stimulus causes short- and long-term morphological and functional neuroprotection against severe PA [27,28]. Fetal asphyctic preconditioning significantly 
decreased the number and density of apoptotic cells in hippocampus (unpublished results), striatum and frontoparietal cortex 8 days after birth compared with animals that were not preconditioned [28]. At six months of age, preconditioned animals showed better locomotor function and memory function than non-preconditioned animals [27]. The underlying mechanisms of the neuroprotective role of fetal asphyctic preconditioning are not clear yet and need more research.

Of note, fetal asphyctic preconditioning can not be used as clinical intervention per se. However, gaining more insights into these mechanisms of brain tolerance might provide new approaches for therapeutical interventions that exploit the induced protective mechanisms.

\section{Inflammation}

Increasing evidence implicates cytokines to play a pathogenic role in neurological injury in the developing human nervous system [29]. Damaged cells initiate a cascade of inflammatory responses, including the activation of microglia, astrocytes, endothelial cells and macrophages which in turn produce inflammatory mediators, like reactive oxygen species, chemokines and cytokines [30]. Not only have cytokines been demonstrated to play an important role in the brain response to asphyxia, they also have been implicated to play a protective role in the induction of ischemic tolerance $[25,31]$. However, the exact role of these cytokines and the time pattern of their expression have not been elucidated yet.

Human data revealed elevated IL- $1 \beta$, IL- 6 and TNF- $\alpha$ levels in cerebrospinal fluid (CSF) from newborn infants with asphyctic encephalopathy [32,33]. Moreover, the concentrations of IL-6, IL-8 and IL-10 have been shown to be upregulated in the sera of asphyxiated neonates compared with those in normal neonates [34]. In these studies, blood and/or CSF were taken from neonates who they suspect to have suffered from an asphyctic insult. So these studies do not indicate the exact nature, the severity or the frequency of the asphyctic insult(s). Moreover, sampling was performed at only one time point after birth [32,33].

Interestingly, Ashdown and coworkers reported decreased levels of TNF- $\alpha$ and IL-1 $\beta$ protein and decreased IL- 6 mRNA in brains of newborn rat pups $2 \mathrm{~h}$ after 
global birth asphyxia [35]. By using a model of global birth asphyxia, they speculated that decreased cytokine brain levels may modulate the central nervous system responses to asphyxia [35].

Different brain cytokine responses may be seen when comparing focal (stroke) and global brain injury. Animal models of focal asphyxia are mostly based on the Rice-Vanucci model, where P7 rats are exposed to unilateral carotid artery occlusion, followed by a period of systemic hypoxia [36]. These studies mainly report increased brain cytokine responses, with rather extensive cerebral cortical and subcortical atrophy and cell death [37,38]. Pro-inflammatory cytokines such as IL- $1 \beta$ and TNF- $\alpha$ are secreted early in the inflammatory response and their levels are increased after brain ischemia, inducing an inflammatory reaction in the brain [38-41]. Monocytes and neutrophils, recruited during cerebral ischemia, enhance the production of IL- 6 in response to stimuli from TNF- $\alpha$ and IL- $1 \beta[32,42]$. IL-6 is a pleiotropic cytokine with both pro- and anti-inflammatory effects and is secreted, together with the anti-inflammatory cytokine IL-10, later in the inflammatory response $[33,42]$. All these cytokines appear to directly modulate the apoptotic process, by promoting differentiation, proliferation and subsequent infiltration by leukocytes $[25,29]$. On the contrary, inflammatory cytokines in hypoxic/ischemic brain damage seem also able to induce beneficial effects. TNF knockout mice were shown to have exacerbated neuron damage after focal cerebral ischemia compared to the controls, suggesting that TNF has a neuroprotective effect on cellular responses after brain injury [43]. Furthermore, rats that have experienced middle artery occlusion and have been injected with IL- 6 significantly show reduced brain damage. This suggests that also IL-6 has a potential role in cell-death inhibition after cerebral ischemia [44].

Irrespective of extensive research concerning cytokine involvement in hypoxia/ ischemia, their exact role remains elusive since cytokines are pleitropic, redundant and multifunctional [45]. The inflammatory response that is observed after hypoxia/ischemia suggests that cytokines such as TNF- $\alpha$, IL- 1 and IL- 6 affect the ceramide metabolism [45]. Ceramides are bioactive sphingolipids, which have emerged as important regulators of cell activation with a wide spectrum of activities 
ranging from the control of cell growth to cell death [46]. Ceramide modulation has been reported in various models of hypoxia/ischemia [47] and is coupled to several pathways that are activated in response to danger signals involving death ligands like TNF- $\alpha$ and may function in concert with other molecular factors in the initiation of hypoxic-ischemic brain damage [45].
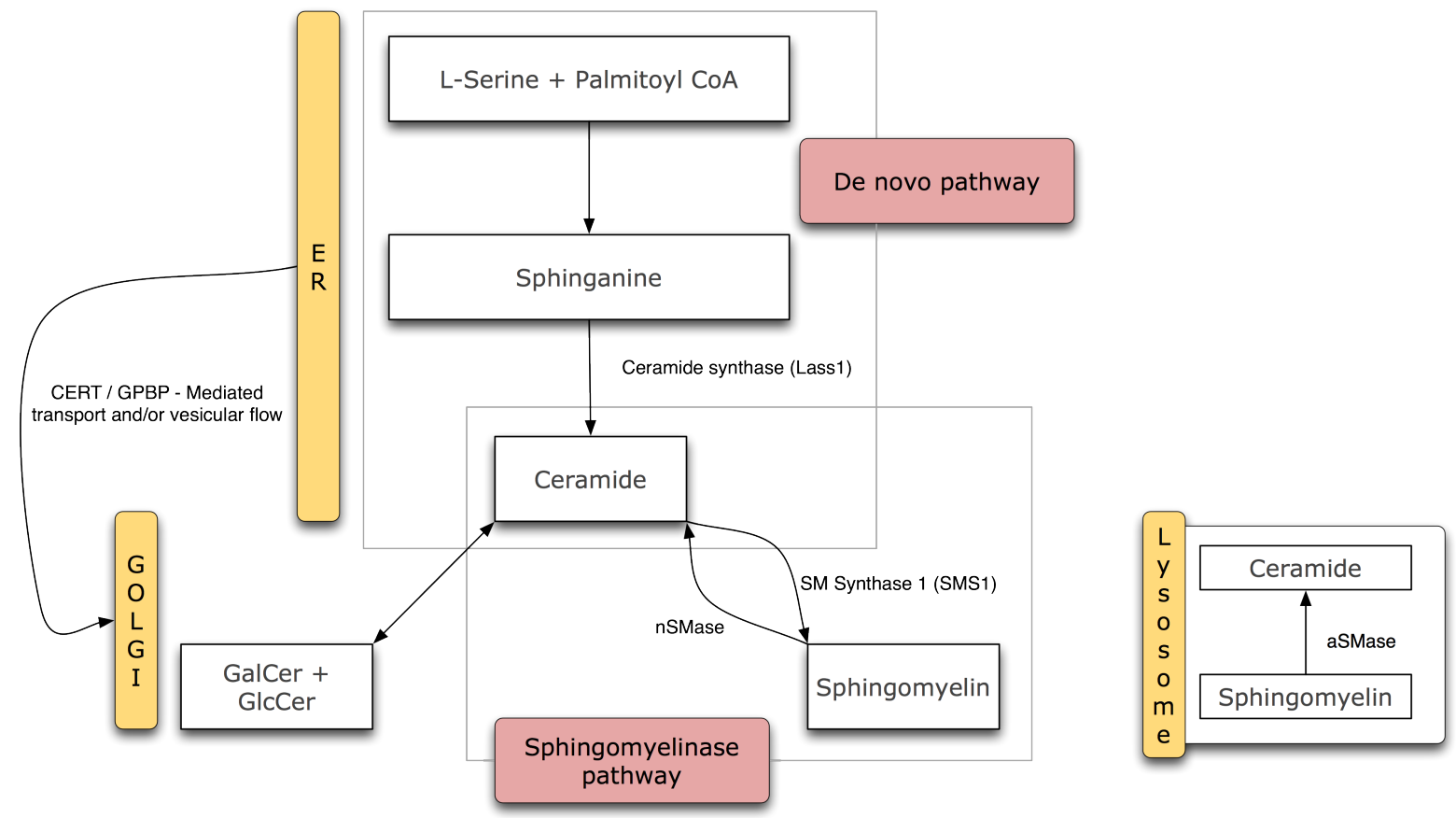

Figure 3: Ceramide generation occurs through three major pathways: (1) de novo synthesis, through condensation of palmitate and serine, (2) hydrolysis of sphingomyelin by sphingomyelinases (SMase) or (3) salvage pathway through breakdown of complex sphingolipids [48]. ER= endoplasmatic reticulum.

Ceramide can be synthesized by three mechanisms: 1) de novo synthesis through the condensation of palmitate and serine, 2) sphingomyelin hydrolysis mediated by the enzyme sphingomyelinase and 3) salvage pathway through breakdown of more complex sphingolipids (Figure 3) [48]. Augmentation of the intracellular ceramide content will have widespread effects, because the ceramide pathway is involved in several signal transducing pathways [49]. Low levels of sphingomyelin and ceramide promote cell proliferation and survival, while high levels of these molecules 
lead to cell damage and/or death [50]. At even higher levels, cellular dysfunction is induced, leading to apoptosis because ceramide activates several pro-apoptotic molecules like the activation of cathepsin D which cleaves Bid and leads to subsequent activation of caspase-9 and caspase-3 [49-51]. Cell responses to ceramide depend upon the engagement of downstream effectors, the cell microenvironment and concomitant activation of enzymes that convert ceramide into other metabolites [52]. In order to have proper ceramide actions, ceramide transporters such as ceramide transporter protein (CERT) and Good-Pasture Binding Protein (GPBP) are very important. These transporters transfer ceramide from the endoplasmatic reticulum (ER) to the Golgi apparatus (Figure 3) [48]. Therefore, low levels of CERT or GPBP, indicating decreased ceramide transportation, can induce stress in the ER by increased accumulation of ceramide into this organelle. High levels of these transporters lead to less ceramide in the ER and ultimately to proliferation [48].

A lot of evidence indicates that ceramide accumulation is initiated by oxidative and metabolic stress, which can be induced for example by anoxia, nutrient deprivation, and exposure to TNF- $\alpha$ [53]. Indeed, ceramide levels have been reported to be upregulated during cerebral ischemia [47] and can either induce or prevent apoptosis in neurons depending on the severity of the insult to the brain [54]. Moreover, studies have shown that inhibition of ceramide production reduces ischemic neuronal injury and improves behavioral outcome [54,55]. A beneficial effect of ceramide has been proposed in the concept of preconditioning. During preconditioning in cardiomyocytes, it has been shown that the levels of ceramide and sphingosine are increased [56]. In primary neuronal rat cultures, it has also been demonstrated that ischemic preconditioning induces significant increases in ceramide levels compared to the control cultures [57]. In addition, Liu et al. have demonstrated that hypoxic-ischemic preconditioning of rat cortical neurons protects them against a severe insult via TNF- $\alpha$ and ceramide mediators [58]. Altogether, these studies suggest that ceramide signaling is surely a promising tool in understanding the inflammatory mechanisms in asphyxia and hypoxic-ischemic preconditioning. 


\section{CHORIOAMNIONITIS}

Besides asphyxia, chorioamnionitis is also known to be a source of antenatal exposure to inflammation. Chorioamnionitis is defined as inflammation of the amniotic fluid and fetal membranes caused by bacteria-produced endotoxin $[59,60]$. Several bacterial species can cause chorioamnionitis and even in the absence of a proven infection, fetuses and neonates may have systemic inflammation referred to as the fetal inflammatory response syndrome (FIRS) [61]. Chorioamnionitis is extremely common and is the leading cause of early preterm delivery and neonatal complications, like adverse pulmonary, neurological and other organ development [62].

\section{Consequences and outcomes}

Chorioamnionitis is involved in the pathogenesis of chronic lung disease with as two major adverse outcomes respiratory distress syndrome, caused by surfactant deficiency and structural lung immaturity, and bronchopulmonary dysplasia [63]. Additionally, diffuse or focal white matter disease, usually described as periventricular leukomalacia, has been shown to be the predominant feature of brain damage in premature newborns of $<32$ weeks of gestation. In clinical studies, antenatal exposure to inflammation has been associated with an increased risk for periventricular leukomalacia and cerebral palsy [62]. Sepsis, shock and multi-organ damage are consequences of chorioamnionitis as well $[62,64]$.

In addition, chorioamnionitis is a major risk factor for spontaneous premature labor [64]. At least $60 \%$ of all preterm births below 25 weeks of gestation affect mothers that suffer from chorioamnionitis [59,65]. Preterm delivered babies are at high risk for neonatal death, and preterm babies that survive have a higher risk of morbidity [66,67]. Complications of preterm birth include neurological deficits, blindness, deafness and chronic lung disease. Survival and adverse cognitive, organ functional and motor outcomes are inversely related to gestational age; the highest rates of adverse outcomes are seen in the extremely preterm (28 weeks of gestational age) and severely preterm (28-31 weeks of gestational age) infants [68]. Even late preterm infants born between 32 and 36 weeks of gestation have an increased risk of adverse birth outcomes, such as feeding intolerance and visual sys- 
tem abnormalities $[66,68]$. Later in childhood, the prematurely born infants have reduced motor, speaking, writing, mathematical and behavioural skills, compared to children born at term [68]. It is therefore not surprising that premature babies require specialized medical care that can become very demanding for the family and expensive for society [67].

\section{Pathophysiology}

Ascending bacterial invasion of the uterine cavity is thought to be the most common route of infection [62]. A variety of pathogens such as Ureaplasma urealyticum, Escherichia coli and Streptococcus agalactiae, have been associated with chorioamnionitis [69]. The bacteria can ascend through the uterine cervix without necessarily rupturing the membranes. The latter step might be due either to the adherence and virulence of the organism itself or to a deficiency in maternal genetic or immunological responses. The bacteria then replicate in the choriodecidual space. At this point, a maternal inflammatory response is initiated in $30 \%$ of the cases. In $70 \%$ of women that suffer from chorioamnionitis, the bacterial invasion of the membranes and placenta not necessarily induces a maternal inflammatory response [70]. Consequently, the clinical course can be distinguished between a systemic inflammatory response of the mother with increased leukocyte counts and fever, and a clinically silent form with no symptoms in the mother. Both forms can result in preterm birth but the predominant form at early gestational age is clinically silent [59]. It has been reported that the incidence of the clinical silent form is up to $60 \%$ throughout different populations and is usually postnatally diagnosed by pathological reviewing of the placenta $[59,68]$.

When the bacteria continue to propagate and cross into the placenta, they can subsequently be transported into the fetus via the umbilical cord. At this stage, a fetal inflammatory response might occur. This so called fetal inflammatory response syndrome (FIRS) is characterized by systemic inflammation and an elevation of pro-inflammatory mediators [61]. Bacteria might also enter the amniotic cavity by crossing the amniotic membranes. Degradation of the amniotic membranes, a result of the inflammatory response, could assist in the transport of bacteria into the amniotic cavity. Bacteria in the amniotic cavity might enter the fetus via swallow- 


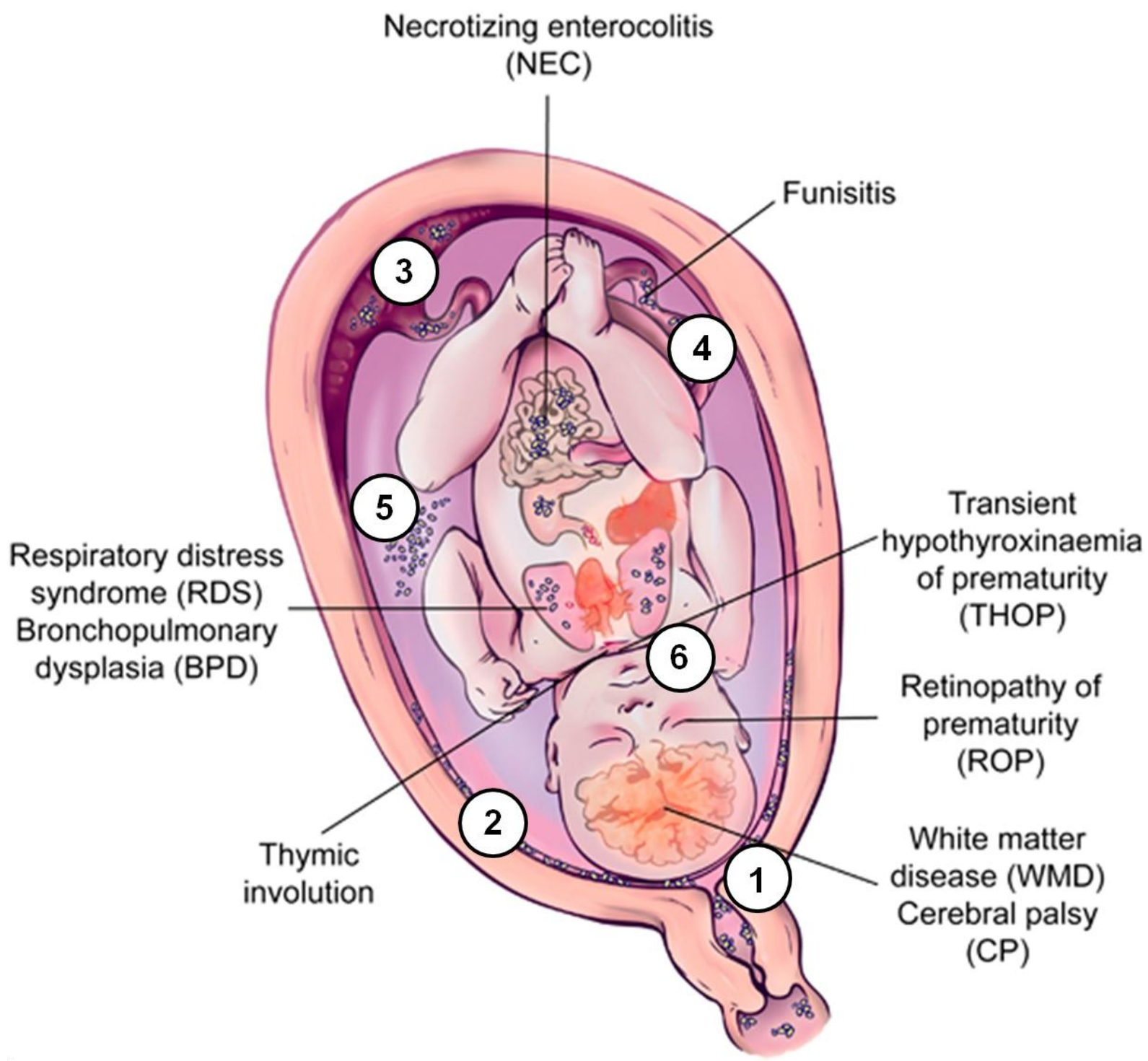

Figure 4: Pathogenesis of chorioamnionitis-induced FIRS. Bacteria may ascend through the uterine cervix (step 1) and replicate in the chorion-decidual space (step 2). The bacteria continue to propagate and cross into the placenta (step 3), and are subsequently transported into the fetus via the umbilical cord (step 4). Bacteria might also enter the amniotic cavity by crossing the amniotic membranes (i.e. going immediately from step 2 to step 5). Bacteria in the amniotic cavity (step 5) might enter the fetus via swallowing (step 6) [71]. The fetus is being exposed to contaminated amniotic fluid leading to multi-organ disease of the fetus (adapted from Gantert et al., 2010). 
ing $[59,71]$. Consequently, the fetus is being exposed to contaminated amniotic fluid which may lead to multi-organ disease of the fetus (Figure 4) [62]. An alternative hypothesis suggests that in most cases the bacteria do not reach the fetus and/or the amniotic cavity; instead, they remain in the choriodecidual space and elicit an inflammatory reaction in the decidua and placenta. In turn, inflammatory mediators (e.g. cytokines and chemokines) reach the fetal circulation and evoke a fetal response $[59,61,62,71]$.

Although the mechanisms whereby chorioamnionitis induces FIRS are poorly understood, it is generally accepted that the production of inflammatory mediators is involved in the preterm birth associated morbidity and even mortality $[65,72]$ (Figure 4).

Several factors related to chorioamnionitis are not very well understood. First, the time course of chorioamnionitis relating to the invasion of microbes is not known [59]. Second, the interaction of the pathogens with the immune system of the host in chorioamnionitis is poorly understood. Moreover, the mechanism that might prevent or control the inflammatory response has not been studied in chorioamnionitis [59]. Third, the function of the immune system may be primed or modulated by previous exposures to pro-inflammatory agonists. For example, the immune system can be rendered tolerant after previous exposures to proinflammatory stimuli [73]. Fourth, the effect of the indolent chorioamniotic infection on the function of the immune cells is not clear [74].

\section{Treatment options}

Treatment for chorioamnionitis includes antimicrobial agents, antipyretics, expedition of delivery and supportive care [75]. Despite the fact that chorioamnionitis is common, there is limited evidence to support one specific antibiotic regimen over another. The standard regimen includes ampicillin and gentamicin [76]. Additional treatments in the management of acute chorioamnionitis include the antipyretic acetaminophen [75]. A study of fetal acid-base balance showed significant improvements in intrapartum fetal heart rate monitoring as well as in bicarbonate and base excess values following treatment of intrapartum fever with acetaminophen [75]. Although delivery should be expedited, caesarean section is still reserved for the 
usual obstetric indications [75]. Surfactant supplementation remains the cornerstone among the therapies in the premature infant. Administration of corticosteroids is associated with lower rates of respiratory distress syndrome, severe intraventricular hemorrhage, and periventricular leukomalacia [76]. Therefore, administering antenatal steroids may be safe and beneficial in reducing perinatal morbidity after preterm birth associated with chorioamnionitis [76,77]. Nevertheless, randomized clinical trials are needed to adress this issue [77].

\section{Inflammation}

The fetal inflammatory response to microorganisms is dominated by neutrophils. Macrophages appear in small numbers days to weeks after the onset of infection [78]. Data from experimental sheep work suggest that the immature fetus can respond to inflammation after exposure to pro-inflammatory stimuli such as IL-1 and LPS [79, 80], with subsequent structural changes suggestive of lung maturation [81]. Inflammatory cell numbers and cytokine concentrations are increased in the fetal lung within $5 \mathrm{~h}$ and recruitment of neutrophils into the fetal airways is detected within $12 \mathrm{~h}$ [82]. Cytokines and inflammatory cells reach their peak after 3 days. Epithelial injury of the fetal lung results in a permeability defect and consequently apoptosis follows by an increase in proliferation during resolution [82]. This injury response is accompanied by an increase of surfactant protein mRNAs, thinning of alveolar wall and increased phospholipid concentrations for several weeks. The lung parenchyma decreases by activation of matrix metalloproteinases. As a result of this inflammatory response, the fetal lung has a more mature lung structure with more surfactant and better compliance improving gas exchange. However, the alveolarization of these fetuses was reduced similar to the mild phenotype of bronchopulmonary dysplasia, showing that the alveolar development can be intrauterinely impaired by inflammation [62].

Also the neuronal inflammatory response after chorioamnionitis has been established in fetal sheep. Chorioamnionitis induced microglial activation and apoptotic cell death in all regions of the ovine central nervous system [83]. Regional responses varied as a function of its maturational stage. Astrocytes were increased in the brain and cerebellum of LPS-exposed fetuses but not in the spinal cord. Ma- 
ture oligodendrocytes decreased in the cerebral and cerebellar white matter, the cerebral cortex, caudate putamen, and hippocampus 14 days after LPS. Neurons in the cerebral cortex, hippocampus, and substantia nigra were reduced 14 days after LPS [83]. The activated microglia in the diffuse white matter lead to the apoptotic death of the developing oligodendrocytes by two mechanisms. First, the diffuse microglial production of proinflammatory cytokines, for example, TNF- $\alpha$, IL-1, IL-6 and interferon- $\gamma$, affects mainly the cerebral fetal white matter oligodendrocytes, and secondarily induces neuronal loss and impaired neuronal guidance, with high levels of pro-inflammatory cytokines. Although low cytokine levels are crucial for the maturation and differentiation of oligodendrocyte precursors into myelinating mature oligodendrocytes, high levels of pro-inflammatory cytokines, as found in inflamed fetal brains, inhibit this process. In addition, pro-inflammatory cytokines may affect the central nervous system by increasing the permeability of the developing blood brain barrier exclusively observed in the cerebral white matter, and by impairing the cerebral blood flow. Second, developing oligodendrocytes are injured by reactive oxygen species and reactive nitrogen species generated by either oligodendrocyte glutamatergic excitotoxicity or microglial activation. The activated microglia generate free radicals and induce the ultimate death of the antioxidant-deficient immature oligodendrocytes. Combined, these two processes result in morphological white matter lesions and the impairment of myelination, one of the most serious complications of white matter disease in preterm infants [62].

In addition to the lungs and brain, also other organs are involved in the inflammatory response to choriamnionitis such as the gut [84], eyes [85] and cardiovascular system $[86,87]$. Although many studies have investigated the inflammatory responses in several organs, only few research focuses on the systemic impact of chorioamnionitis [61]. Especially the involvement of the fetal liver has thus far not been studied. Since the liver is a central organ in an organism's metabolism and damage to the liver may play an important role in the development of metabolic diseases $[88,89]$, exploring the hepatic inflammatory response to chorioamnionitis is an important aspect for further investigation. 


\section{ANIMAL MODELS}

The choice of an appropriate animal model is not straightforward since many animal species differ from humans in e.g. the length of gestation, the number of fetuses, the type of placentation, the hormonal regulation of parturition and the timing of fetal organ maturation. For example, little mammalian species such as rabbits, rats and mice have short gestational periods with multiple fetuses and they experience maturational sequences only near term or postnatally [66]. Among all species, sheep and nonhuman primates are closest to the human development, since the maturation of the lungs and cerebrum, two organs with important contributions to neonatal morbidity and mortality most closely parallel the development in humans [66]. As would be expected, nonhuman primates represent a near-ideal species; however, ethical considerations limit the use of this animal model [71].

\section{Rat model for global perinatal asphyxia and fetal asphyctic preconditioning}

Many rat models have been established to study the pathophysiological processes leading to neonatal HIE after asphyxia. When using rats as model for perinatal asphyxia we should take into account the postnatal brain maturation and the relatively small body size by which it is difficult to monitor multiple organ failure. Nevertheless, the rat is very often used in experimental asphyxia studies as they are easily used for functional and behavioral testing, an absolute necessity for preclinical therapeutic trials. Moreover, because of the short longevity of these animals, long-term follow-up studies can be performed in an adequate period of time [90].

The most widely used rat model for perinatal brain damage is the Rice-Vannucci model. In this model, hypoxia-ischemia is induced in 7-day-old rats by unilateral carotid artery occlusion followed by systemic exposure to hypoxia (8\% oxygen) [91]. Brain damage in this model is restricted to the ipsilateral hemisphere of the occlussion and is observed in the cortex, the subcortical and periventricular white matter, the striatum, and the hippocampus [26,36]. Although this model is excellent to study physiological and molecular mechanisms of hypoxic-ischemic brain damage, no global asphyxia with multi-organ failure is induced. Therefore, 
this model is more appropriately considered as a model for perinatal stroke. In addition, the model lacks the transitional physiology from intra-uterine to extra-uterine life.

A model for global perinatal asphyxia was introduced by Bjelke and coworkers [92], in which asphyxia is induced at birth by removing the uterine horns, still containing the pups, and submersing these in a water bath at $37^{\circ} \mathrm{C}$ for various periods of time. The severity of the brain damage in this model is dependent on the duration of the asphyctic insult, with $100 \%$ survival at 16 min of asphyxia and almost $100 \%$ mortality at $21 \mathrm{~min}$ of asphyxia $[92,93]$. Although the rat brain maturation at the time of birth is different from that of humans, this model reflects the asphyctic insult that occurs during the transition from fetus to newborn in a time of dynamic physiological adaptation.

The rat model used in this thesis is therefore based on the global perinatal asphyxia model of Bjelke and coworkers. At the day of birth, after the vaginal delivery of the first born pup, Caesarean section (C-section) is performed. To induce PA, pregnant rats are euthanized and the uterine horns containing the pups are placed in saline $\left(0,9 \% \mathrm{NaCl}, 37^{\circ} \mathrm{C}\right)$ for exactly $19 \mathrm{~min}$. Afterwards, pups exposed to the PA insult, are delivered and stimulated manually to breathe in a closed incubator ( $37^{\circ} \mathrm{C}$ and $75 \%$ air humidity). To assess the effects of neuroprotection, induced by fetal asphyctic preconditioning, we induce global fetal asphyxia at embryonic day E17. This procedure is based on a model of Cai and coworkers [94], where the uterine and ovarian arteries are clamped during $30 \mathrm{~min}$. The beneficial effects of fetal asphyctic preconditioning using this model have already been described by Strackx and coworkers [27, 28] and this model was further used in this thesis to elucidate the inflammatory mechanisms.

\section{Sheep model for chorioamnionitis}

Animal models using pregnant mice, rats, rabbits, sheep and nonhuman primates have all been utilised as models for chorioamnionitis [5]. In these animal models numerous infectious and inflammatory agents, including killed or live Escherichia coli, group B streptococci, components of the cell wall of bacteria, IL-1 and atypi- 
cal bacteria such as Ureaplasma, have been used. Which of these approaches better approximates what occurs in the human situation is not clear. The application of live bacteria might mimic those cases of human preterm birth in which cultures of amniotic fluid test positive for bacteria. However, studies that performed PCR for detection suggest that there is an inflammatory, but not an overtly infectious state, in the uterus, decidua or placenta [71]. The use of killed bacteria, components of the cell wall or pro-inflammatory cytokines create an inflammatory state in the absence of an overt infection [71].

Several routes of administration of the infectious or pro-inflammatory agents are described and can be classified in systemic and localized models of infection or inflammation. Systemic models, where the infectious or inflammatory agents are administered intraperitoneal to the mother, might represent more accurately human conditions that promote bacteremia or sepsis. Although these models can provide insights into the mechanisms that promote preterm birth in the setting of systemic illness, they might not provide sufficient insight into the specific pathways that are triggered. Models of localized inflammation can mimic more accurately what is observed clinically, since most individuals with chorioamnionitis do not have signs or symptoms of systemic illness, nor do they demonstrate significant increases in white blood cell counts, C-reactive protein or temperature. These models of local inflammation can be subdivided in intra-amniotic, intrauterine and intracervical [5,71].

In this thesis, we will make use of an established clinical relevant sheep model for chorioamnionitis $[67,82,95]$. In this model endotoxin, a lipopolysaccharide (LPS) from cell walls of gram negative bacteria, was injected as provocative stimulus since it is a pro-inflammatory agonist that can be used to study inflammatory responses [96]. The LPS is administered into the amniotic cavity assuming that the stimulus and not maternal mediators (i.e. cytokines) are directly triggering a fetal response, which in turn might signal delivery. 


\section{AIM AND OUTLINE OF THE THESIS}

The aim of this thesis was to gain more insights into the mechanisms of the inflammatory responses to perinatal asphyxia and chorioamnionitis. Chapters 2, 3, 4 and $\mathbf{5}$ are dedicated to elucidate the inflammatory mechanisms during perinatal asphyxia and fetal asphyctic preconditioning. The studies regarding chorioamnionitis are reported in chapters 6 and 7.

In chapter 2, we investigated in the brain the involvement of pro- and antiinflammatory cytokines in the induction of asphyctic tolerance to perinatal asphyctic injury. To further determine which pathways are of importance during the inflammatory response to fetal asphyctic preconditioning, we assessed ceramide metabolism in total brain, which is described in chapter 3. At 6 months of age, fetal asphyxia preconditioned animals had improved locomotor function and object memory after perinatal asphyxia compared with non-preconditioned animals [27]. Therefore, in chapter 4, we analyzed if these long-term functional consequences could reflect changes in inflammatory cytokines and ceramides in 8-month-old animals. Finally, we demonstrated in chapter 5 that the liver was also influenced by fetal and perinatal asphyxia indicating the global impact of asphyxia. The liver was also the organ of interest in our sheep model of chorioamnionitis, since information regarding the impact of chorioamnionitis on the liver is limited. In chapter 6 we showed that chorioamnionitis induced prenatal liver inflammation, leading to metabolic disturbances in the fetus. Moreover, these chorioamnionitis-mediated antenatal liver disturbances have long-lasting postnatal effects on lipid metabolism (chapter 7). Finally, chapter 8 discusses the major findings of this thesis and addresses possible future implications of the results. 



\section{2}

Fetal asphyctic preconditioning modulates the acute cytokine response thereby protecting against perinatal asphyxia in neonatal rats

Evi VLASSAKS, Eveline STRACKX, Johan S. H. VLES, Pilar MARTINEZ-MARTINEZ, Boris W. KRAMER and Antonio W.D. GAVILANES 


\section{ABSTRACT}

Background Perinatal asphyxia (PA) is a major cause of brain damage and neurodevelopmental impairment in infants. Recent investigations have shown that experimental sublethal fetal asphyxia (FA preconditioning) protects against a subsequent more severe asphyctic insult at birth. The molecular mechanisms of this protection have, however, not been elucidated. Evidence implicates that inflammatory cytokines play a protective role in the induction of ischemic tolerance in the adult brain. Accordingly, we hypothesize that FA preconditioning leads to changes in the fetal cytokine response, thereby protecting the newborn against a subsequent asphyctic insult.

Methods In rats, FA preconditioning was induced at embryonic day 17 by clamping the uterine vasculature for $30 \mathrm{~min}$. At term birth, global PA was induced by placing the uterine horns, containing the pups, in a saline bath for 19 min. We assessed, at different time points after FA and PA, mRNA levels of several cytokines and related receptors in total hemispheres of fetal and neonatal brains.

Results Prenatally, FA induced decreased IL- $1 \beta$, TNF- $\alpha$ and IL-10 mRNA levels, and increased IL- 6 mRNA levels in fetal brains compared with controls. Receptors of investigated cytokines were mostly increased at later time points than their ligand. Interestingly, all pro-inflammatory cytokines decreased $2 \mathrm{~h}$ after birth in all pups that experienced FA and/or PA. The anti-inflammatory cytokine IL-10, however, increased $2 \mathrm{~h}$ after PA. When pups were FA preconditioned, IL-10 mRNA levels were comparable to controls. The same expression pattern was found for IL-6 mRNA levels at $6 \mathrm{~h}$ and $12 \mathrm{~h}$ after birth.

Conclusion FA leads to downregulation of the acute neuro-inflammatory response. This modulation of the cytokine response probably results in the protective inflammatory phenotype seen when combining FA and PA. Elucidating the inflammatory pathways involved in FA preconditioning may have significant implications for preventing post-asphyctic perinatal encephalopathy. 


\section{INTRODUCTION}

Perinatal hypoxia/ischemia or asphyxia is associated with a high mortality rate and causes multi-organ deficits leading to permanent structural brain damage, including encephalopathy $[8,97]$. Irrespective of the prevalence of perinatal asphyxia (PA), there is no effective treatment for term infants having post-asphyctic encephalopathy, except for hypothermia for moderate cases [98]. We have shown in a rat model that a brief episode of fetal asphyxia (FA) at embryonic day 17 (E17) lowers the severity of a subsequent more severe global asphyctic insult at term birth (FA preconditioning) [27]. FA preconditioned animals have less apoptosis in different regions of the brain and show improved locomotor function and object memory after PA compared with non-preconditioned animals $[27,28]$. The molecular mechanisms underlying FA preconditioning have however not been elucidated yet.

Several studies suggest that inflammation has a key role in perinatal brain injury, including cerebral hypoxia/ischemia [35, 99]. Inflammatory cells, such as neutrophils and macrophages but also resident brain cells as astrocytes and microglia have been found to be activated by cerebral ischemia. These cells consequently produce inflammatory mediators, including reactive oxygen species and cytokines [30]. Human data revealed elevated interleukin (IL)-6 and tumor necrosis factor (TNF)- $\alpha$ levels in cerebrospinal fluid (CSF) from newborn infants with asphyctic encephalopathy [32]. Moreover, the concentrations of IL-6, IL-8 and IL-10 have been shown to be upregulated in the sera of asphyxiated neonates compared with those in normal neonates [34]. In experimental stroke models, where they studied the P7 carotid artery ligation model, they also found increased levels and activity of IL-1 $\beta$, IL-6 and TNF- $\alpha$ several hours to days after the insult [37,38]. In contrast, Ashdown and coworkers reported lower IL- $1 \beta$ and TNF- $\alpha$ protein levels and lower IL-6 mRNA levels in neonatal rat brains $2 \mathrm{~h}$ after global birth asphyxia [35]. Although special attention is needed concerning the controversies reported between focal and global asphyctic insults, all these experiments indicate that cytokines may modulate asphyctic damage in brain tissue. Moreover, inflammatory cytokines are implicated to be protective in ischemic brain tolerance [25, 31, 100, 101]. It has been suggested that preconditioning ameliorates asphyxia-induced brain damage 
by suppressing the inflammatory response $[25,31]$. No experimental studies, however, have examined the role of cytokines after global FA preconditioning in a perinatal setting. Therefore, we aimed to investigate the inflammatory response that is activated during asphyctic brain damage. In addition, we investigated whether levels of pro- and anti-inflammatory cytokines modulate neuroprotection against PA injury. To study this, we used a rat model where two global, instead of focal, asphyctic insults were combined to better reflect the pathology of global asphyctic encephalopathy and multiple organ failure in the human neonate [27,28]. We found that FA induces time-dependent cytokine changes, probably resulting in a protective inflammatory phenotype when followed by PA.

\section{MATERIALS AND METHODS}

\section{Animals and tissue processing}

Animal experiments were approved and performed according to the guidelines of the Animal Ethics Board of Maastricht University, the Netherlands. Timedpregnant Sprague-Dawley rats, obtained from Charles River (France), were housed individually under standard laboratory conditions. Pregnant rats were randomly assigned to an experimental group. Unsexed fetuses and male neonates were used within this study.

For the collection of tissue samples, dams and pups were sacrificed by decapitation at several time points after FA and at different time points after birth $(n=4-5)$ (Figure 1). Total brain hemispheres were collected from the offspring, snap-frozen in liquid nitrogen and preserved at $-80^{\circ} \mathrm{C}$ for further analysis.

\section{FA preconditioning and PA}

The current study used a rat model where two global asphyctic insults were combined. At E17, FA preconditioning was induced by performing a midline laparotomy in pregnant rats. Both uterine horns containing the pups were exposed and the uterine and ovarian arteries were clamped using four removable clamps. After 30 min, the clamps were removed to allow reperfusion, the uterine horns were placed 
back intra-abdominally and the abdominal cavity was closed. The procedures explained above were performed in a controlled environment at $37^{\circ} \mathrm{C}$ and $75 \%$ air humidity.

To assure full-term pregnancy, Caesarean section (C-section) was only performed after the vaginal delivery of the first born pup. Control and FA pups were delivered immediately by $\mathrm{C}$-section. To induce PA, pregnant rats were euthanized by decapitation to avoid the potential effect of the anesthetic. After hysterectomy, the uterine horns containing the pups were placed in saline $\left(0,9 \% \mathrm{NaCl}, 37^{\circ} \mathrm{C}\right)$ for exactly 19 min. Afterwards, pups exposed to the PA insult, were delivered and stimulated manually to breathe in a closed incubator $\left(37^{\circ} \mathrm{C}\right.$ and $75 \%$ air humidity). The umbilical cords were ligated and cut to separate the pups from their placentas. Pups were randomly cross-fostered with surrogate dams (maximally 12 pups each dam), which had given birth vaginally on the same day.

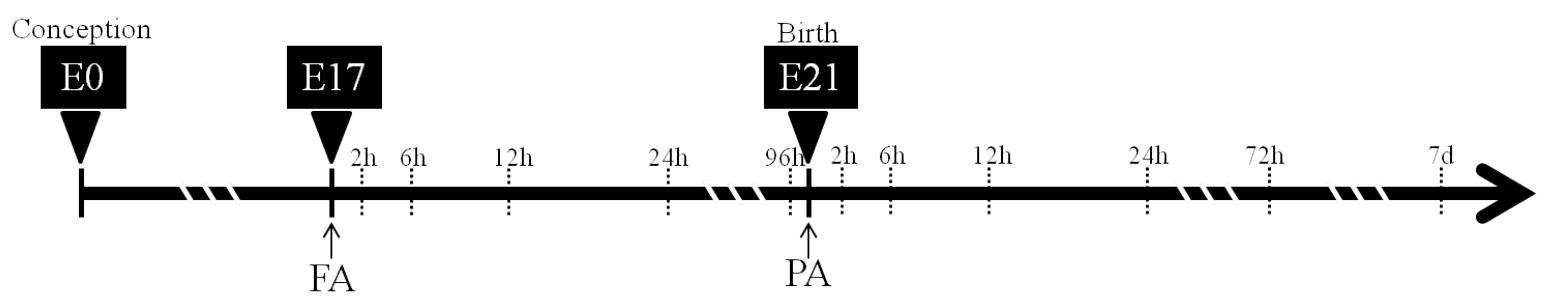

Figure 1: Experimental design. FA was induced at E17 by clamping the uterine vasculature during $30 \mathrm{~min}$. At term birth, global PA was induced by placing the uterine horns containing the pups in a saline bath for $19 \mathrm{~min}$. All animals were delivered by Caesarean section. Pups were euthanized at five different time points after FA prenatally $(n=5$ per group per time point) and six different time points postnatally $(n=4$ per group per time point). $\mathrm{E}=$ embryonic day, $\mathrm{FA}=$ fetal asphyxia, $\mathrm{PA}=$ perinatal asphyxia.

\section{RNA extraction and RT-PCR}

Total RNA was extracted from frozen tissue by homogenization of the samples with Trizol Reagent (Invitrogen, Breda, the Netherlands), according to manufacturer's guidelines. Quality and quantity of the RNA were determined with the Nan- 
odrop (ND-1000 spectrophotometer; Thermo Scientific, Wilmington, USA). RIN values were determined using the Agilent 2100 Bioanalyzer (Agilent Technologies, Amstelveen, the Netherlands) and RNA samples with RIN $>8$ were included. Reverse transcription was carried out from $1 \mu \mathrm{g}$ total RNA using the Revert Aid First Strand cDNA Synthesis Kit (Fermentas, St. Leon Rot, Germany) according to manufacturer's instructions. $5 \mu \mathrm{l}$ of diluted cDNA (dilution 1:20) was amplified with LightCycler 480 SYBR Green I Master (Roche Applied Science, Almere, the Netherlands) in a final volume of $20 \mu \mathrm{l}$. The real-time PCR was performed on a LightCycler 480 system (Roche Applied Science) (45 cycles: $20 \mathrm{~s}$ at $95^{\circ} \mathrm{C}$, $15 \mathrm{~s}$ at $60^{\circ} \mathrm{C}, 15 \mathrm{~s}$ at $72^{\circ} \mathrm{C}$ ). Each PCR was carried out in duplicate and samples negative for RevertAid Reverse Transcriptase were used as negative control. Investigated genes were: IL- $1 \beta$ and IL-1R1 and 2, IL-6 and IL-6R, TNF- $\alpha$ and TNFR1/p55 and 2/p75, IL-10 and IL-10R (Table 1). To standardize for the amount of cDNA, $\beta$-actin, Hypoxanthine-guanine phosphoribosyltransferase (HPRT) and Glyceraldehyde 3-phosphate dehydrogenase (GAPDH) were used as housekeeping genes (Table 1). Values are reported relative to the geomean mRNA of the three housekeeping genes. Quantification cycle values were extracted with the Lightcycler 480 software (Conversion LC and Linge PCR) and calculated based on the cycle threshold $(\mathrm{Ct})$ values.

\section{Statistical analysis}

Statistical analysis was performed with Statistical Package for Social Sciences (SPSS 17.0 Software). Normality was tested using the Kolmogorov-Smirnov test. All results were normally distributed. Prenatal data were analyzed using Student's t-test. All postnatal data were analyzed using one-way analysis of variance (ANOVA) test, followed by post-hoc comparisons using LSD correction. Multivariate testing was performed to test the interaction between birth and asphyxia. Results are presented as mean + SEM and p-values $<0.05$ were considered statistically significant. 
Table 2.1: Oligonucleotide primers used for RT-PCR

\begin{tabular}{|l|l|l|}
\hline Primer & Tm $^{\circ}$ & Sequence (5'-3') \\
\hline \hline HPRT for & 64.1 & TTGCTGGTGAAAAGGACCTC \\
\hline HPRT rev & 64.0 & TCCACTTTCGCTGATGACAC \\
\hline$\beta$-actin for & 64.2 & TTGCTGACAGGATGCAGAAG \\
\hline$\beta$-actin rev & 64.0 & TGATCCACATCTGCTGGAAG \\
\hline GAPDH for & 63.4 & CTCCCATTCTTCCACCTTTG \\
\hline GAPDH rev & 63.8 & ATGTAGGCCATGAGGTCCAC \\
\hline IL-1 $\beta$ for & 67.0 & TACCTATGTCTTGCCCGTGGAG \\
\hline IL-1 $\beta$ rev & 67.6 & ATCATCCCACGAGTCACAGAGG \\
\hline TNF- $\alpha$ for & 64.0 & TGCCTCAGCCTCTTCTCATT \\
\hline TNF $\alpha$ rev & 63.2 & GGGCTTGTCACTCGAGTTTT \\
\hline IL-6 for & 62.5 & AAAGCCAGAGTCATTCAGAGC \\
\hline IL-6 rev & 63.8 & GAGCATTGGAAGTTGGGGTA \\
\hline IL-10 for & 64.0 & CCTGCTCTTACTGGCTGGAG \\
\hline IL-10 rev & 63.8 & TGTCCAGCTGGTCCTTCTTT \\
\hline IL-1R1 for & 63.7 & TTGTGTTTAAGCTGTTGCCG \\
\hline IL-1R1 rev & 62.4 & GTAACCTCGATGGTATCTTCCC \\
\hline IL-1R2 for & 61.9 & AGCACGTTTATCTCAGTGGC \\
\hline IL-1R2 rev & 65.5 & GTGACTGGATCAAAAATCAGCG \\
\hline TNFR1/p55 for & 61.9 & AGTCTACTGTGCCGATATCCC \\
\hline TNFR1/p55 rev & 64.9 & CTTTGACAGGAGCTGAATCCC \\
\hline TNFR2/p75 for & 64.0 & AAATGCAAGCACAGATGCAG \\
\hline TNFR2/p75 rev & 64.3 & CAGCAGACCCAGAGTTGTCA \\
\hline IL-6R for & 63.8 & CTGGTTCCTCTCCTCACCC \\
\hline IL-6R rev & 64.9 & TGTTGCTGTTGTCATTAGGGC \\
\hline IL-10R for & 63.8 & ATTTCACCGTGACCAACCTC \\
\hline IL-10R rev & 64.1 & CCAGGATGTGAATGTCATCG \\
\hline & & \\
\hline
\end{tabular}




\section{RESULTS}

The impact on the cytokine expression of FA prenatally and in combination with a subsequent PA insult postnatally was assessed over a longitudinal time pattern until P7 (Figure 1). Prenatally, after FA, a time-dependent cytokine profile was observed, starting with an acute response of IL- $1 \beta$ and TNF- $\alpha$ and followed by the response of IL-10 and IL-6. All receptors for indicated cytokines showed mainly an elevation in mRNA levels after FA, mostly at time points after their respective cytokine response. Postnatally, all pro-inflammatory cytokines (IL- $1 \beta$, TNF- $\alpha$ and IL-6) decreased $2 \mathrm{~h}$ after birth, regardless of the severity of the insult (FA, PA or combination). The anti-inflammatory cytokine IL-10 increased $2 \mathrm{~h}$ after severe PA. However, in FA preconditioned rats this effect was attenuated, showing an inflammatory phenotype similar to control animals. The same expression pattern was seen later for IL-6 mRNA at $6 \mathrm{~h}$ and $12 \mathrm{~h}$ after birth. From P3 onwards, no significant differences were found for any cytokines compared with control levels (data not shown).

\section{Acute downregulation of IL-1 $\beta$ and TNF- $\alpha$ mRNA levels but increased IL-6 levels after fetal asphyxia}

Prenatally, FA induced an acute downregulation of IL- $1 \beta$ at $2 \mathrm{~h}$ (Figure $2 \mathrm{~A} ; \mathrm{p}=0.008$ ) and TNF- $\alpha$ at $6 \mathrm{~h}$ (Figure 2D; $\mathrm{p}=0.05$ ) compared with the respective controls. Variable mRNA levels were found for the receptors of both cytokines. The functional signaling receptor IL-1R1 decreased $12 \mathrm{~h}$ after FA (Figure $2 \mathrm{~B}, \mathrm{p}=0.047$ ). The decoy receptor for IL-1 $\beta$ (IL-1R2) showed increased mRNA levels $6 \mathrm{~h}$ and $96 \mathrm{~h}$ after FA compared with controls (Figure $2 \mathrm{C} ; \mathrm{p}=0.01$ and $\mathrm{p}=0.003$ respectively). At $24 \mathrm{~h}$ after FA, however, IL-1R2 levels were decreased (Figure $2 \mathrm{C} ; \mathrm{p}=0.007$ ). A trend towards increased TNFR1/p55 levels was seen $12 \mathrm{~h}$ after FA (Figure 2E; $\mathrm{p}=0.09$ ), while TNFR2/p75 showed a significant increase at $6 \mathrm{~h}$ after FA (Figure $2 \mathrm{~F} ; \mathrm{p}=0.009$ ), a tendency towards higher levels $24 \mathrm{~h}$ after the FA insult (Figure 2F; $\mathrm{p}=0.06$ ) and increased levels at $96 \mathrm{~h}$ after FA (Figure 2F; $\mathrm{p}=0.03$ ). 
A

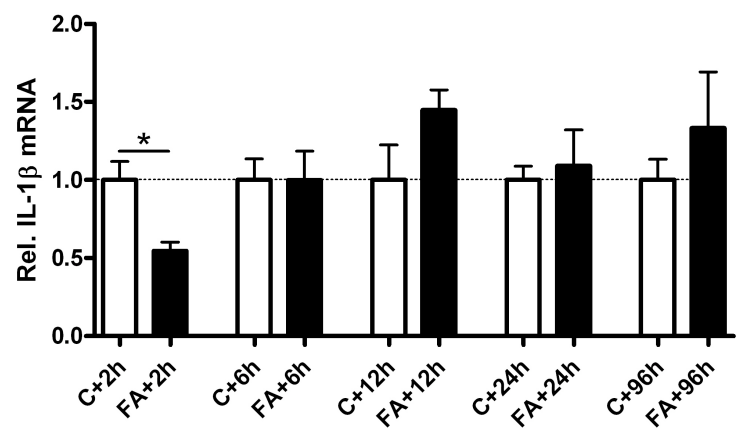

C

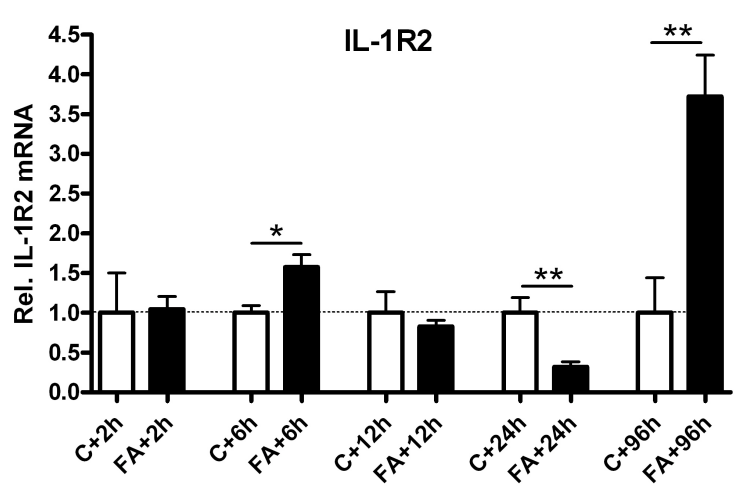

$\mathbf{E}$

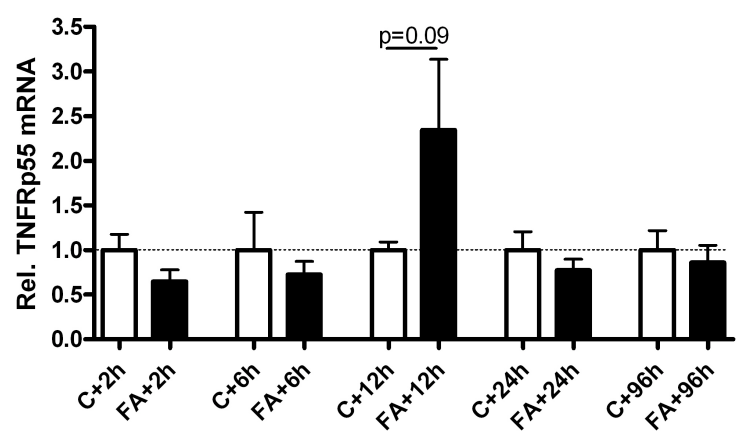

B

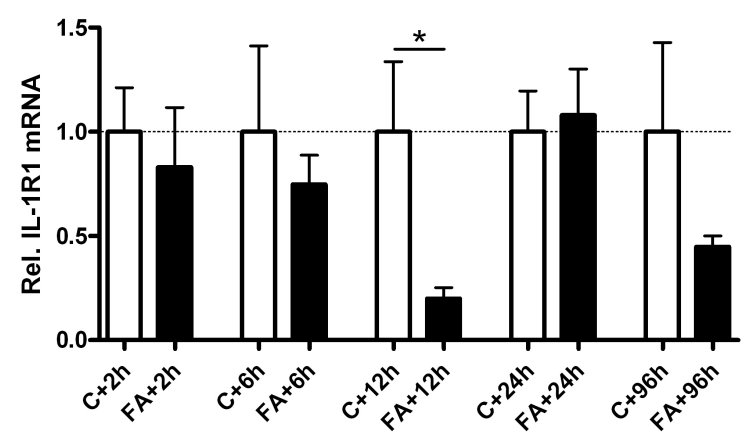

D

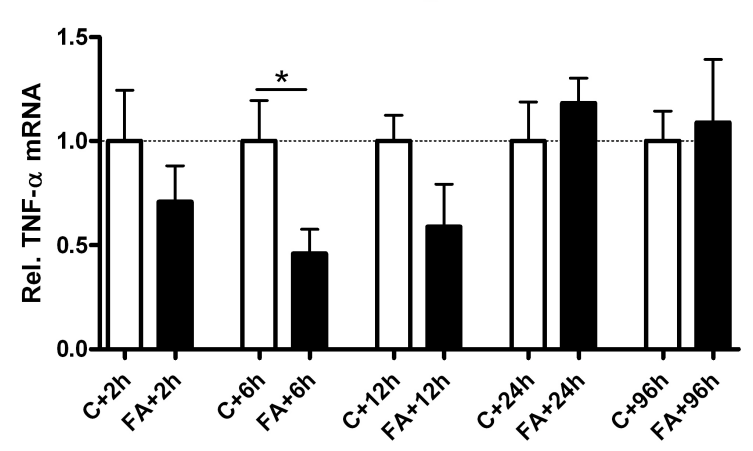

$\mathbf{F}$

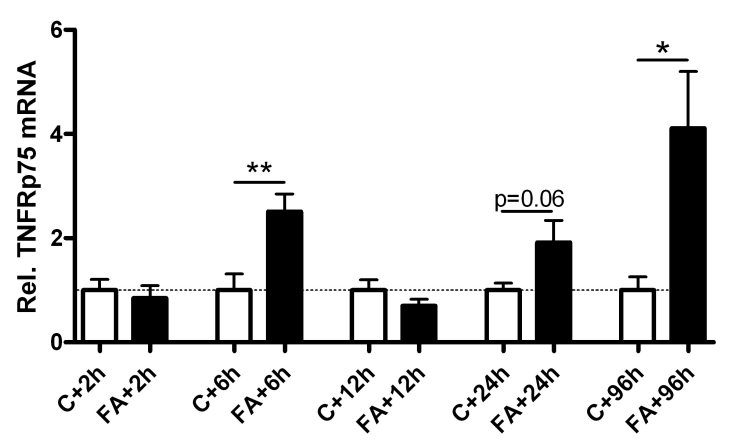

Figure 2: IL-1 $\beta$ and TNF- $\alpha$ are decreased after FA with differential receptor expression at several time points after FA. Prenatal relative mRNA levels of IL-1 $\beta$ (A), IL-1R1 (B), IL-1R2 (C), TNF- $\alpha$ (D), TNFR1/p55 (E) and TNFR2/p75 (F) in control and FA animals. mRNA levels are relative to the geomean of measured housekeeping genes and normalized to control levels. $* \mathrm{p}<0.05$ and $* * \mathrm{p}<0.01$ significantly different from respective control group. Data shown as mean + SEM. 
The anti-inflammatory cytokine IL-10 followed the same pattern as the acute inflammatory response of IL- $1 \beta$ and TNF- $\alpha$, with decreased IL-10 mRNA levels at $12 \mathrm{~h}$ after FA (Figure 3A; $\mathrm{p}=0.05$ ). In contrast to the decreased levels of the other cytokines measured in this study, IL-6 mRNA levels were elevated $96 \mathrm{~h}$ after FA compared with controls (Figure $3 \mathrm{C} ; \mathrm{p}=0.034$ ). The IL-6R showed no significant changes after FA (Figure 3D).
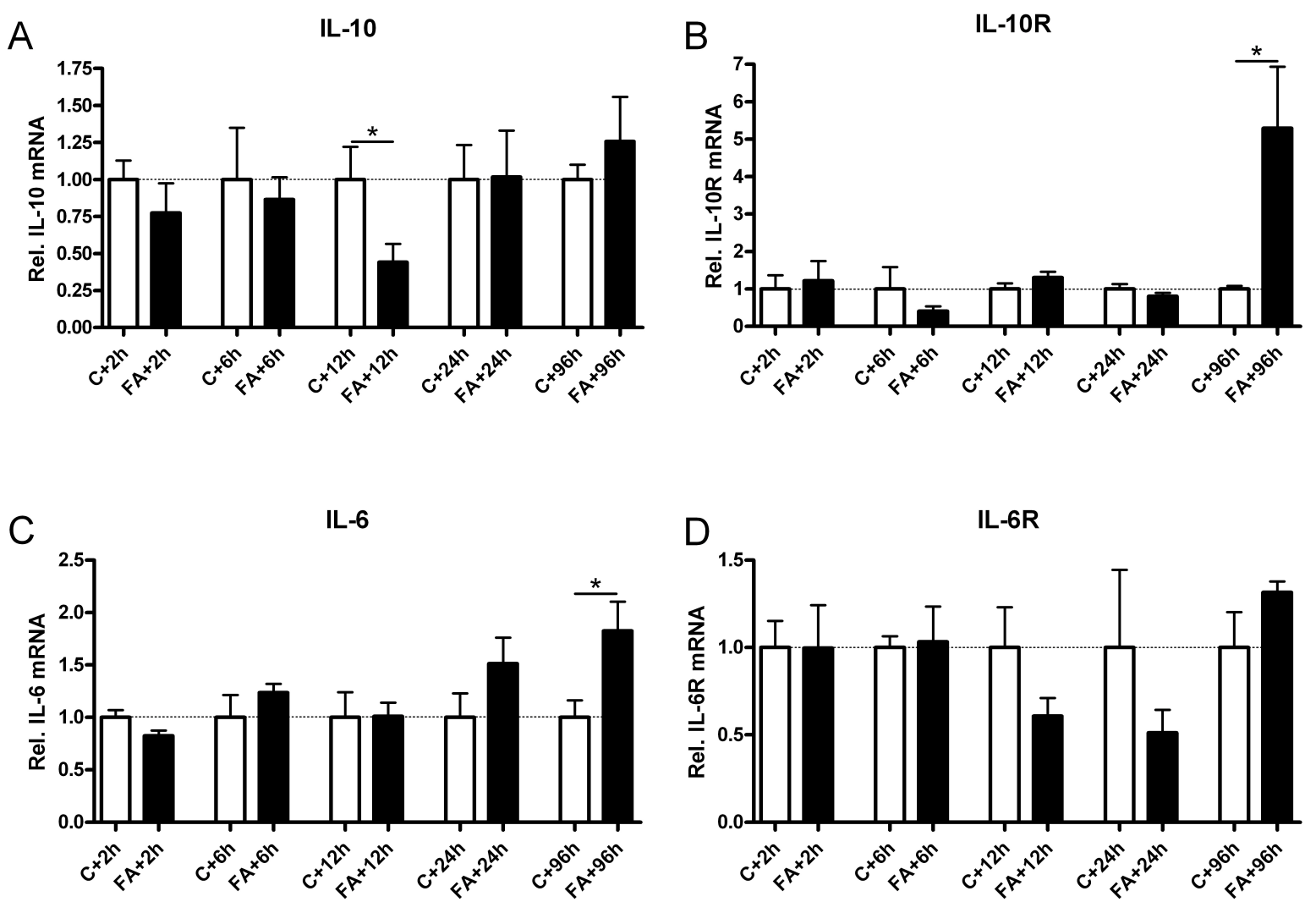

Figure 3: Downregulated IL-10 mRNA $12 \mathrm{~h}$ after FA, but increased IL-10R and IL-6 levels $96 \mathrm{~h}$ after FA. Prenatal relative mRNA levels of IL-10 (A), IL-10R (B), IL-6 (C) and IL-6R (D) in control and FA animals. mRNA levels are relative to the geomean of measured housekeeping genes and normalized to control levels. $* \mathrm{p}<0.05$ significantly different from respective control group. Data shown as mean + SEM. 


\section{An asphyctic insult acutely downregulates the pro-inflammatory response $2 \mathrm{~h}$ after birth}

Postnatally, IL- $1 \beta$, TNF- $\alpha$ and IL- 6 mRNA levels decreased $2 \mathrm{~h}$ after birth in all experimental groups compared with control levels (Figure 4A-C; $p<0.05$ ). To investigate if the decrease in pro-inflammatory cytokines $2 \mathrm{~h}$ after birth was attributed to the effect of birth itself or if it was specifically due to asphyxia, we compared cytokine levels from control E21 animals with control P0 animals ( $2 \mathrm{~h}$ after birth).
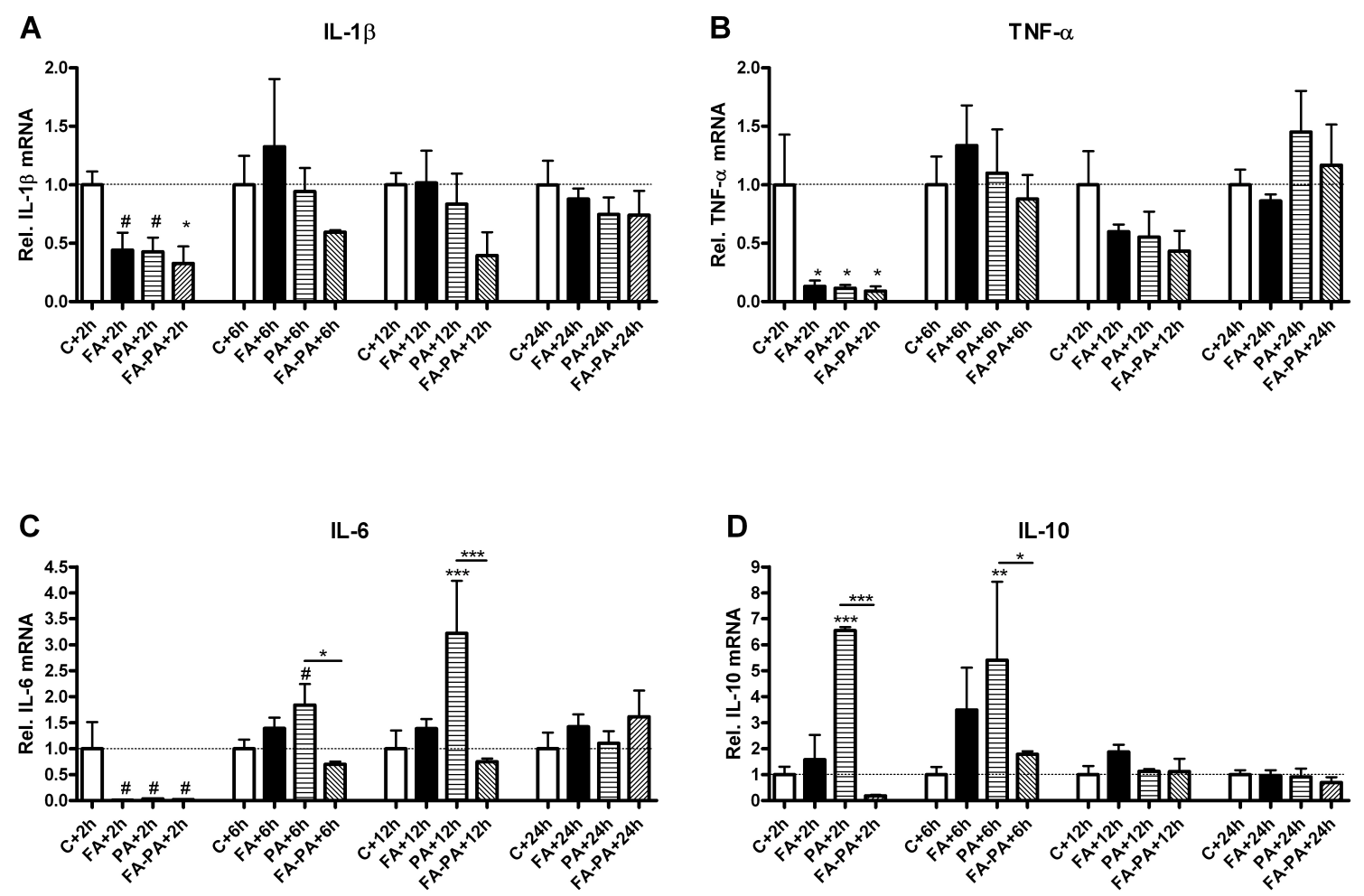

Figure 4: Acute downregulation of the pro-inflammatory response $2 \mathrm{~h}$ after birth in all experimental groups. Increased IL-10 and IL-6 mRNA levels in non-preconditioned PA animals, but baseline IL-10 and IL-6 mRNA levels in preconditioned animals. Postnatal relative mRNA levels of IL-1 $\beta$ (A), TNF- $\alpha$ (B), IL-6 (C), and IL-10 (D) in control, FA, PA and FAPA animals. mRNA levels are relative to the geomean of the measured housekeeping genes and normalized to control levels. \# $0.07<\mathrm{p}<0.05$; * $\mathrm{p}<0.05$ and $* * \mathrm{p}<0.01$ indicate significant differences between groups. Data shown as mean + SEM. 
Comparisons revealed that IL-1 $\beta$ and IL-10 decreased due to birth (Figure 5A+B; $\mathrm{p}<0.01$ and $\mathrm{p}<0.001$ respectively). mRNA levels for TNF- $\alpha$ and IL-6, however, did not differ between E21 and P0 animals ( (Figure $5 \mathrm{C}+\mathrm{D}) ; \mathrm{p}=0.12$ and $\mathrm{p}=0.47$ respectively). Hence, we studied the mRNA levels of the housekeeping genes to assess if the decrease in IL- $1 \beta$ and IL-10 was attributed to a more general downregulation of the cellular response.

Figure 5E shows that the housekeeping genes increased $2 \mathrm{~h}$ after birth in comparison with the E21 animals (left panel; $\mathrm{p}<0.001$ ). Moreover, the housekeeping genes were not affected by an asphyctic insult (Figure 5E, right panel). These results indicate that IL- $1 \beta$ and IL-10 decreased due to the birth process and that this response is specific for these cytokines. However, because IL- $1 \beta$ levels were even lower in animals that experienced an asphyctic insult, we assessed if a synergic effect between birth and asphyxia was present. Multivariate testing between prenatal (96 h after FA) and postnatal ( $2 \mathrm{~h}$ after birth) animals revealed that for TNF- $\alpha$, IL-6 and IL-10 the birth process interacts with the asphyctic insults $(\mathrm{p}<0.05)$. Moreover, the postnatal downregulation of the pro-inflammatory cytokines (IL- $1 \beta$, TNF- $\alpha$ and IL-6) in the animals that were previously exposed to an asphyctic insult is specifically attributed to the asphyxia itself irrespective of the nature of the insult (FA and/or PA).

\section{IL-10 and IL-6 levels are increased after perinatal asphyxia but attenuated in FA preconditioned animals}

Postnatally, IL-10 dissociated from the observed pro-inflammatory response with increased mRNA levels $2 \mathrm{~h}$ after the PA insult. This effect was attenuated in the preconditioned animals (FA+PA group) (Figure 4D; $<<0.001$ ). Interestingly, at 6 $\mathrm{h}$ and $12 \mathrm{~h}$ after birth a similar IL-6 mRNA profile as for IL-10 was observed. More specifically, at $6 \mathrm{~h}$ IL-6 mRNA levels were reaching almost statistical significance in animals previously exposed to PA (Figure $4 \mathrm{C} ; \mathrm{p}=0.06$ ). Animals that were also exposed to the FA preconditioning stimulus (FA+PA group), however, showed baseline IL- 6 mRNA levels (Figure $4 C ; p=0.05$ for PA vs. FA-PA). This effect was even stronger expressed at $12 \mathrm{~h}$ with increased IL-6 mRNA in PA animals (Figure $4 \mathrm{C} ; \mathrm{p}<0.001$ ), and baseline IL-6 levels in preconditioned animals (Figure 4C; $\mathrm{p}<0.001$ for PA vs. FA-PA). 

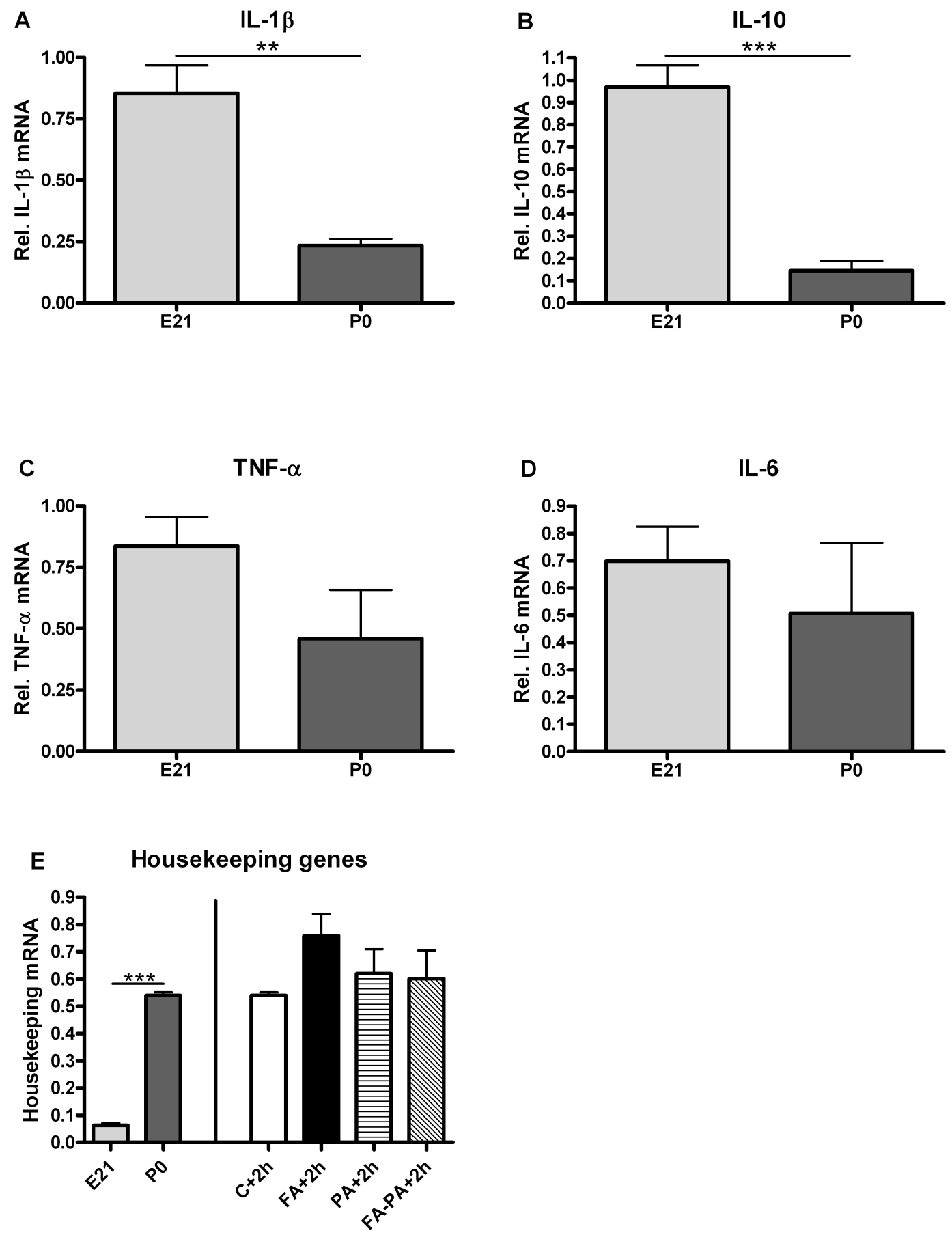

Figure 5: Comparison between E21 and P0 cytokine response. mRNA levels of IL$1 \beta$ (A), IL-10 (B), TNF- $\alpha$ (C) and IL-6 (D) are presented relative to the geomean of measured housekeeping genes. mRNA levels of housekeeping genes from control animals at E21 and P0 (left panel) and housekeeping mRNA levels at $\mathrm{P} 0$ in all groups (right panel) (E). ** $\mathrm{p}<0.01$ and $* * *$ $\mathrm{p}<0.001$ indicate significant differences between groups. Data shown as mean + SEM. 


\section{DISCUSSION}

In this study, we aimed to elucidate the inflammatory cytokine profile in response to global PA. Moreover, we investigated whether pro- and anti-inflammatory cytokines played a role in the induction of neuroprotection against PA injury. Prenatally, a time-dependent cytokine profile was observed after FA with acute downregulation of IL-1 $\beta$, TNF- $\alpha$ and IL-10; followed by increased IL- 6 mRNA levels at a later stage. The main observation of our study is that postnatally, PA rats showed higher IL-10 and IL-6 mRNA levels after PA than control rats. Moreover, in FA preconditioned rats this effect was attenuated, showing an inflammatory phenotype similar to control animals.

Cytokines have been demonstrated to play an important role in the brain response to asphyxia. Moreover, they have been implicated to play a protective role in the induction of ischemic tolerance [25,31]. The exact role of these cytokines and the time pattern of their expression have, however, not been elucidated yet. In human studies, increased IL- $1 \beta$, IL- 6 and TNF- $\alpha$ have been found to be upregulated in CSF of asphyxiated newborns compared with control neonates [32,33]. In these studies, CSF sampling was performed at only one time point $(24 \mathrm{~h}$ and $48 \mathrm{~h}$ respectively) after birth. More time points are included in experimental models of focal asphyxia. These models are mainly based on the Rice-Vanucci model [36], where P7 rats are exposed to unilateral carotid artery occlusion, followed by a period of systemic hypoxia. Although these models are widely used to investigate the mechanisms involved in PA, they miss the transitional physiology from intra-uterine to extra-uterine life. The birth process itself is a physiological unique period, with specific hormonal changes priming the fetus to be born [102]. This aspect is covered by the model used in our study as we awaited the vaginal delivery of the first pup so that the physiological conditions that occur during labour are mimicked.

One aim of our study was to investigate the inflammatory mechanisms in global asphyxia. To the best of our knowledge, the role of cytokines in preconditioning during the fetal period has not been studied before. Therefore, we explored the im- 
pact of 30 min sublethal global fetal asphyxia on the mRNA levels of IL- $1 \beta$, TNF$\alpha$, IL-10 and IL-6. Additionally, we assessed the mRNA levels of corresponding receptors to study the regulation of these cytokine pathways.

Interestingly, a time-dependent cytokine response after FA was observed prenatally. This response started $2 \mathrm{~h}$ after FA, with decreased IL- $1 \beta$ levels. IL- $1 \beta$ is a key mediator of the acute inflammatory response and has been shown to be upregulated $1 \mathrm{~h}$ as well as at later time points (6-24 h) after global cerebral ischemia in adult rats [40]. Also in a neonatal setting, IL-1 $\beta$ is upregulated 3-6 h after the hypoxic/ischemic insult $[38,41]$. Important differences with the addressed studies and our observations include the nature and the time point of the asphyctic insult. The FA that we induce is only a sublethal insult with no fetal losses or morbidity in adulthood [27]. Additionally, we induce the stimulus prenatally and it has been suggested that the fetal brain is less reactive to inflammatory reactions than a mature brain [41]. Evidence supports that blocking IL-1 $\beta$ expression by injecting anti-IL1 $\beta$ antibodies, leads to decreased brain infarction [103]. Therefore, the general time-dependent cerebral pro-inflammatory downregulated response due to FA could play a protective role in the injured brain of these fetuses. To get more insights into the final cytokine response, we also studied mRNA levels of IL-1R1 and IL-1R2. IL-1 $\beta$ binding to IL-1R1 leads to increased inflammation by NF $\kappa B$ activation, while IL-1R2 acts as a decoy receptor preventing inflammation [104]. Our results suggest that IL-1R1 is not stimulated after FA. Moreover, IL-1R2 is upregulated $6 \mathrm{~h}$ and $96 \mathrm{~h}$ after FA, suggesting that IL-1 $\beta$ cannot exert its biological effects as it is captured by IL-1R2. This supports our speculation that the down regulated IL- $1 \beta$ response to FA could play a protective role in these fetuses.

The decrease in IL- $1 \beta$ was followed by a drop in TNF- $\alpha$ levels at $6 \mathrm{~h}$ after FA. It is widely accepted that IL- $1 \beta$ induces TNF- $\alpha$ and both share the same physiological effects [39]. However, the exact role of TNF- $\alpha$ in the pathogenesis of asphyxia remains controversial as TNF- $\alpha$ has been shown to exert both toxic and neurotrophic effects [105]. TNF- $\alpha$ protects neurons by stimulating antioxidant pathways [43], plays a role in neuronal tissue repair [106] and can induce tolerance to ischemic brain damage [107]. In contrast, inhibition of TNF- $\alpha$ reduced cerebral ischemic damage [108] and TNF- $\alpha$ knock-out mice showed less neuronal 
damage after focal cerebral ischemia [43]. This dual role can be attributed to the functioning of both TNF-receptors. TNFR $1 / \mathrm{p} 55$ induces apoptosis by activating the caspase cascade, while TNFR2/p75 promotes cell proliferation and survival by inducing the activation of the mitogen-activated protein kinase p38 pathway [106]. In our study, both TNF receptors showed increased expression patterns but at different time points after FA. Especially TNFR2/p75 seems to play an important role after FA as it was increased at $6 \mathrm{~h}, 24 \mathrm{~h}$ and $96 \mathrm{~h}$ after FA. Again, we speculate that mild FA shifts the balance to an anti-inflammatory response since TNF- $\alpha$ can promote ischemic tolerance [109] and activation of TNFR2/p75 has been shown to induce anti-inflammatory pathways [110].

IL-10 is an important anti-inflammatory mediator of the immune response and has been shown to be both upregulated [111] and downregulated [112] after an hypoxic-ischemic insult. In addition, IL-10 prevents neurodegeneration by protecting neurons through activation of PI-3-kinase and STAT-3 pathways [113,114]. In our study, we detected decreased IL-10 mRNA levels $12 \mathrm{~h}$ after FA. The same issues as addressed for IL- $1 \beta$ concerning the nature and the time point of the asphyctic insult apply for IL-10. Moreover, as IL-10 is responsible for downregulating IL- $1 \beta$ and TNF- $\alpha[113,114]$, this function could be inhibited after mild FA because mRNA levels of IL- $1 \beta$ and TNF- $\alpha$ were already low at earlier time points.

Although it is known that IL- $1 \beta$ and TNF- $\alpha$ are early mediators inducing IL-6 production $[32,42]$ and that IL- 6 is secreted together with the anti-inflammatory cytokine IL-10 [33,42], IL-6 mRNA was not decreased but upregulated $96 \mathrm{~h}$ after FA. IL-6 is a pleiotropic cytokine with both pro- and anti-inflammatory effects in the injured brain $[115,116]$. IL- 6 has been shown to be higher expressed on neurons in response to ischemia, promoting neuronal survival and development [117]. Furthermore, a study by Yamashita and coworkers showed that endogenous IL-6 plays a critical role in preventing apoptosis of damaged neurons in the acute phase of cerebral ischemia in mice [118].

Altogether, the acute downregulated response of IL- $1 \beta$ and TNF- $\alpha$ may be associated with anti-inflammatory properties, consequently leading to decreased IL-10 levels. Late elevation of IL-6 may trigger repair mechanisms indicating a beneficial inflammatory effect after mild FA. 
The second aim of our study was to investigate whether the changes in cytokine response observed due to FA have any protective effect against a subsequent more severe PA insult. Our data showed decreased mRNA levels of the pro-inflammatory cytokines (IL- $1 \beta$, TNF- $\alpha$ and IL-6) $2 \mathrm{~h}$ after birth in all experimental groups compared with control animals. This decrease in cytokine levels is in accordance with a study from Ashdown et al., who observed decreased IL- $1 \beta$ and TNF- $\alpha$ protein and decreased IL-6 mRNA in the brain at $2 \mathrm{~h}$ after birth asphyxia [35]. They claimed that the downregulation in cytokine levels was due to global birth asphyxia itself and not to the $\mathrm{C}$-section. Comparison of E21 and P0 control animals revealed that IL-1 $\beta$ and IL-10 levels decreased due to birth. Control levels of TNF- $\alpha$ and IL-6 did not show decreased levels due to birth. These are remarkable findings because studies have shown that the pro-inflammatory response is increased during the birth process [119]. Additionally, although IL-1 $\beta$ control levels decreased $2 \mathrm{~h}$ after birth, these levels were lower in the experimental groups than in the control groups suggesting an interaction between the birth process and asphyxia. However, a synergic effect between birth and any asphyctic insult (FA and/or PA) was not observed for IL- $1 \beta$ but for the other cytokines. Hence, the drop in inflammatory cytokines $2 \mathrm{~h}$ after birth remains an interesting and important observation because focal asphyctic episodes have been associated with increased inflammation [37,38,41,99]. Studies indicate that endogenous production of glucocorticoids may play an important role in the suppression of specific cytokine production and inflammation [120]. As it is established that birth is associated with higher stress hormones such as cortisol and catecholamines [121] and hypoxia induces an even higher catecholamine response in the fetus [122], we may assume that the animals that had experienced an asphyctic insult before have higher corticosteroid levels. Accordingly, these animals would be able to downregulate the pro-inflammatory response as a protective mechanism.

One of the main findings of our study is that IL-10 was highly expressed in asphyxiated animals $2 \mathrm{~h}$ after birth. Moreover, significant elevated mRNA levels of IL- 6 were observed at $6 \mathrm{~h}$ and $12 \mathrm{~h}$ after PA. These data are comparable with the abundance of evidence indicating increased cytokine levels in the brain due to hypoxia-ischemia $[37,38,41,99]$. Most importantly, a preconditioning 
effect was observed both in IL-10 and IL-6 mRNA levels. So the increase in IL-10 and IL-6 mRNA caused by PA was attenuated and comparable to control levels in the preconditioned FA-PA animals. Studies have already demonstrated that inhibition of cytokine activity reduces excitotoxic brain injury in neonatal rats $[37,123,124]$. These studies, however, are mainly focused on the effects of IL-1 $\beta$ and TNF- $\alpha$. In our study, we could not observe a significant contribution of IL- $1 \beta$ and TNF- $\alpha$ in preconditioning during postnatal life. This supports recent investigations showing that neuroprotection can be present without affecting IL-1 $\beta$ and TNF- $\alpha$ production $[125,126]$. An important role for the cytokines IL10 and IL-6 after global asphyxia is highlighted in our study. An IL-6 response to asphyxia has been correlated with higher mortality rates [33]. Therefore, the changes in IL-6 levels could indicate why FA preconditioned animals have higher survival chance after PA compared with non-preconditioned animals. Although both human and experimental data show increased levels of IL-10 and IL-6 after asphyctic injury $[32,37,38,42,112]$, the precise role of these cytokines in cerebral asphyxia has not been fully elucidated. Both cytokines are considered to have anti-inflammatory properties, promoting neuronal survival and reducing ischemic damage $[113,114,116]$. Hence, further studies on the role of IL-10 and IL-6 in the pathophysiology of global perinatal brain injury are warranted.

\section{CONCLUSIONS}

In the present study, we report important findings concerning the inflammatory mechanisms of global asphyctic brain damage and the role of inflammatory cytokines in the induction of neuroprotection against perinatal asphyctic injury. To our knowledge, we are the first who report cytokine changes in a clinical relevant rat model for global PA in a longitudinal design. Moreover, we also investigated the cytokine response after FA, highlighting cytokine levels during fetal life. Consistent with our hypothesis, we demonstrated that FA induces time-dependent cytokine changes with acute downregulation of IL- $1 \beta$, TNF- $\alpha$ and IL-10; followed by increased IL-6 mRNA levels later on. Importantly, these prenatal changes downregulate the cytokine response when combined with a global asphyctic insult at birth. 
PA rats show higher IL-10 and IL-6 mRNA levels than control rats. However, in FA preconditioned (FA+PA) rats this effect was attenuated, showing an inflammatory phenotype similar to control animals. Therefore, more insights into the inflammatory pathways of IL-6 and IL-10 may have significant implications for preventing global post-asphyctic encephalopathy. 



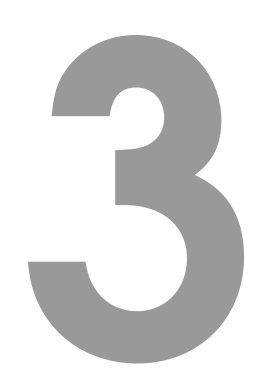

\section{Fetal asphyxia induces changes in the ceramide pathway and in brain immunological responses}

Evi VLASSAKS, Chiara MENCARELLI, Maria NIKIFOROU, Eveline STRACKX, Maria J. FERRAZ, Johannes M. AERTS, Marc H. DE BAETS, Pilar MARTINEZMARTINEZ and Antonio W.D. GAVILANES

Submitted to Journal of Lipid Research 


\section{ABSTRACT}

Fetal asphyctic (FA) preconditioning, induced by a brief episode of experimental hypoxia-ischemia, offers neuroprotection to a subsequent more severe asphyctic insult at birth. Extensive cell stress and apoptosis are important contributing factors of damage in the asphyctic neonatal brain. Since ceramide acts as a second messenger for multiple apoptotic stimuli including hypoxia/ischemia, we sought to investigate the possible involvement of ceramide signaling genes in endogenous neuroprotection, induced by FA preconditioning. Global FA was induced in rats by clamping both uterine and ovarian vasculature for 30 minutes. FA resulted in an increase of brain ceramide/sphingomyelin anabolic enzymes such as ceramide synthase and sphingomyelin synthase 1, leading to changes in sphingolipid metabolite concentrations at $96 \mathrm{~h}$ post FA. This observation correlated with increased apoptosis and inflammation. After birth, ceramide and sphingomyelin levels remained high in FA subjected animals, indicating that a long term regulation of the ceramide pathway is involved in the mechanisms inducing tolerance to a subsequent, otherwise lethal, asphyctic event. 


\section{INTRODUCTION}

Perinatal asphyxia (PA; hypoxia/ischemia) is one of the most common causes contributing to neonatal morbidity and mortality [127, 128]. During periods of asphyxia, impaired substrate delivery occurs leading to energy failure [129]. As a consequence, asphyctic newborns develop multi-organ failure [12], with the brain as one of the most affected organs reflecting severe and long-term cognitive and motor deficits $[8,14]$. Yet, no effective therapies are available, except for hypothermia in term infants suffering from moderate encephalopathy [19]. There is, however, evidence that molecular mechanisms of organ preconditioning result in tissue tolerance to a following more severe insult that would otherwise be lethal [20]. Previously, we have shown in a rat model that fetal asphyxia (FA), induced at embryonic day 17 (E17) by clamping the uterine vasculature during 30 min, induces brain tolerance to a stronger PA insult $[27,28]$. Moreover, less TUNEL-positive cell numbers were seen in brains of FA preconditioned animals compared to nonpreconditioned animals subjected to PA [28]. Hence, the identification of the signaling pathways and effectors involved in brain tolerance is of primary importance for the development of new neuroprotective therapies.

Important mechanisms may involve changes in ceramide levels which have been reported in various models of hypoxia/ischemia [47, 57]. Ceramide is the common structure of all complex sphingolipids and is a very active metabolite [47]. Increased ceramide synthesis in response to short periods of hypoxia induces DNA fragmentation and cell death $[47,130]$. Conversely, chronic exposure to hypoxia is characterized by the lack of ceramide accumulation [131] suggesting that a protective adaptive response to chronic hypoxia may exist.

Since ceramide acts as a second messenger in activating cell signaling pathways involved in apoptosis [132], its production is highly regulated and its levels inside the cell depend on the activity of several enzymes that participate in its synthesis and catabolism $[47,132,133]$ (Figure 1). Little is known about how ceramide and its metabolites are regulated during FA preconditioning and subsequent neuroprotection. In the current study, we aim to investigate changes occurring in the sphingomyelin/ceramide pathway after a sublethal FA insult to identify molecules 
important in brain tolerance. Similarly, we study the crosstalk between ceramide metabolism and the neuronal inflammatory and apoptotic response. Better understanding of these mechanisms will allow the development of new neuroprotective therapies.
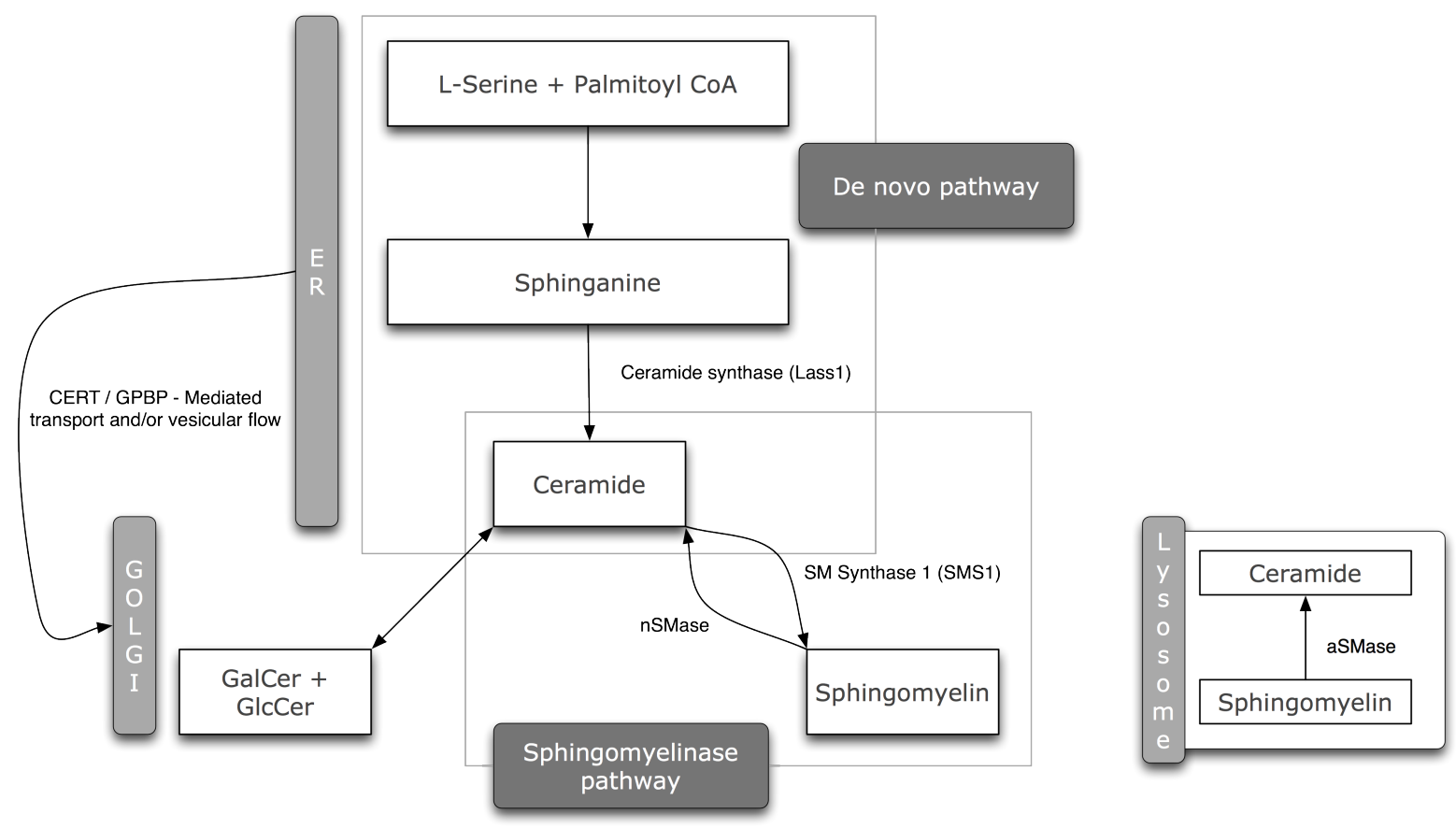

Figure 1: Ceramide metabolism pathway. Ceramide can be generated by the activity of two general pathways. (1) The de novo pathway that occurs at the cytosolic surface of the endoplasmatic reticulum (ER) from condensation of L-serine with palmitoyl-coA. Once formed, ceramide is delivered to the Golgi apparatus and converted to sphingomyelin by SMS1 (Sphingomyelinase pathway). (2) The catabolic pathway, that takes place at the membrane of different organelles in which ceramide is formed by degradation of sphingomyelin by different sphingomyelinases (SMases). 


\section{EXPERIMENTAL PROCEDURES}

\section{Animals}

All experiments were approved by the Animal Ethics Board of Maastricht University on animal welfare according to Dutch governmental regulations. All efforts were made to minimize the pain and stress levels experienced by the animals and to minimize the number of rats necessary to produce reliable scientific data. Timed-pregnant Sprague-Dawley rats (E14; Charles River, France) were kept under standard laboratory conditions (food and water given ad libitum, $21-22^{\circ} \mathrm{C}$ environment temperature and a $12 \mathrm{~h}$ light/dark schedule). Unsexed fetuses and male neonates were used within this study.

\section{Experimental design}

FA was induced as previously described by Strackx et al [27]. Shortly, at E17 FA was induced by clamping both uterine and ovarian arteries with removable clamps for $30 \mathrm{~min}$ (Figure 2A). At time of birth, all pups were born by caesarian section. Afterwards the pups were randomly cross-fostered to surrogate dams (max. 12 pups per mother). Animals were sacrificed at $6 \mathrm{~h}, 12 \mathrm{~h}, 72 \mathrm{~h}$ and $96 \mathrm{~h}$ after the FA insult and $2 \mathrm{~h}, 6 \mathrm{~h}$ and $7 \mathrm{~d}$ after birth ( $\mathrm{n}=6$ for each group at every time point) (Figure 2B). Total brains of fetal and neonatal pups were collected and snap-frozen. All samples were stored at $-80^{\circ} \mathrm{C}$ prior to further analysis.

\section{RNA isolation}

Total RNA was extracted from frozen tissue by homogenization of the samples with Trizol Reagent (Invitrogen, Breda, the Netherlands) according to manufacturer's guidelines. Quality and quantity of the RNA were determined using a Nanodrop ND-1000 spectrophotometer (Thermo Scientific, Wilmington, MA). RIN values were determined using the Agilent 2100 Bioanalyzer (Agilent Technologies, Amstelveen, the Netherlands) and RNA samples with RIN $>8$ were included. 
A

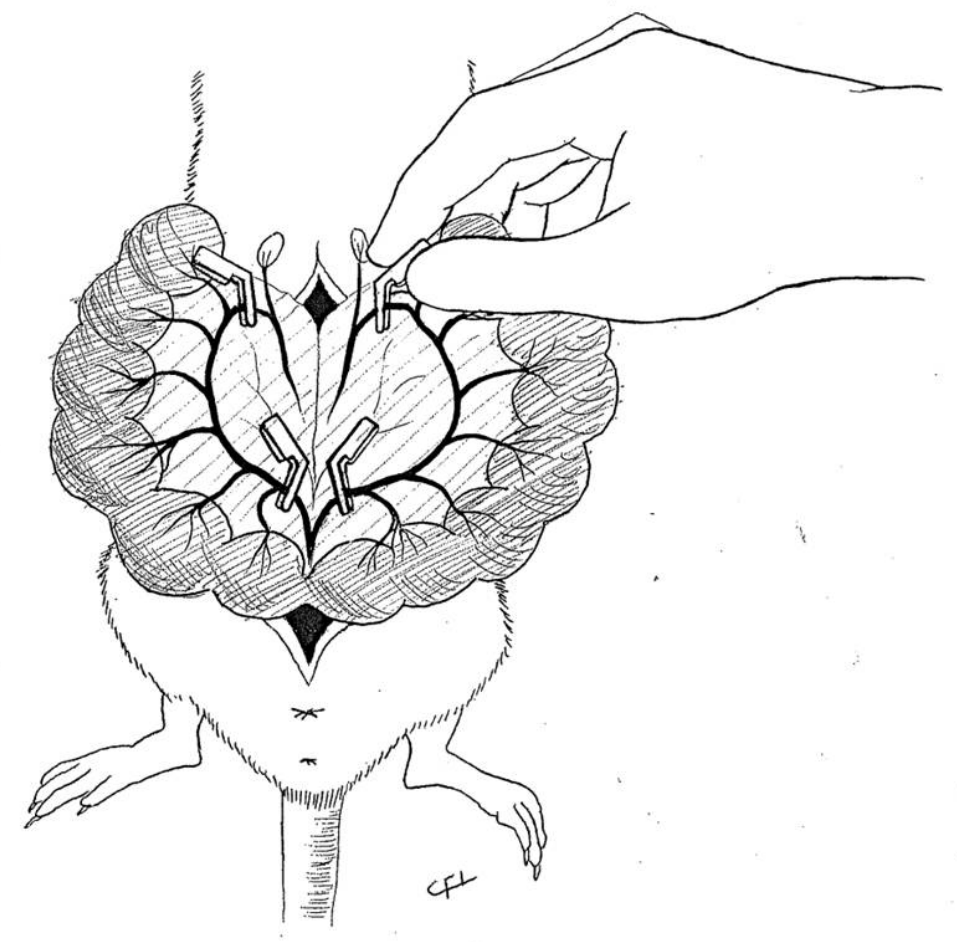

B

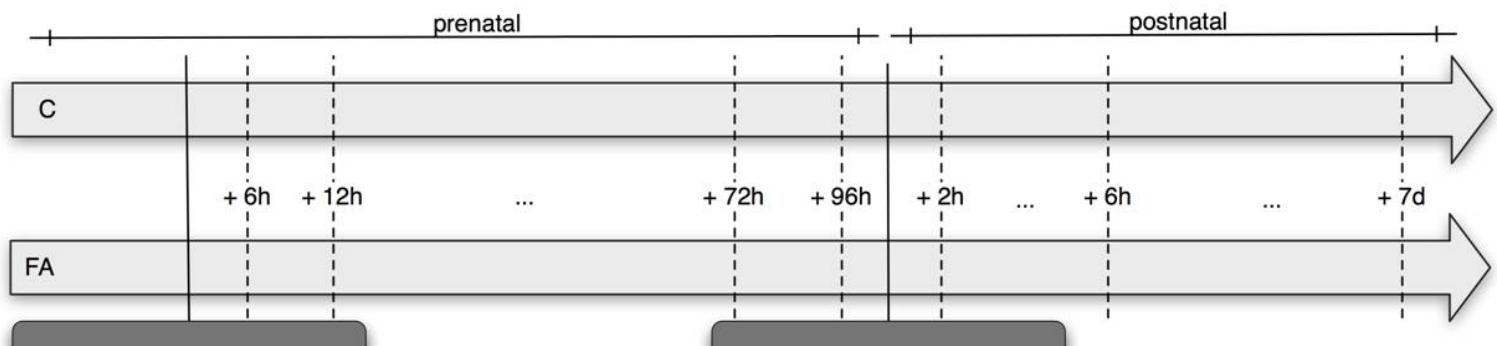

E17: Fetal asphyxia

Birth

Figure 2: Experimental design. FA was induced at E17 by clamping the uterine vasculature during $30 \mathrm{~min}$ (A). At term birth, animals were delivered by Caesarean section. Pups were euthanized at four different time points after FA prenatally and three different time points postnatally ( $\mathrm{n}=6$ per group per time point) (B). $\mathrm{C}=$ control, $\mathrm{E}=$ embryonic day, $\mathrm{FA}=$ fetal asphyxia. 


\section{Reverse transcription}

cDNA was synthesized by using the RevertAid First Strand cDNA synthesis kit (Fermentas, St. Leon Rot, Germany) according to the manufacturer's protocol. The samples were diluted 1 to 20 with DEPC-treated sterilized water and stored at $-80^{\circ} \mathrm{C}$ prior to quantitative PCR analysis.

\section{Real-time PCR}

Primers were designed using Primer3plus (http://www.bioinformatics.nl/cgi-bin/ primer3plus/ primer3plus.cgi, Boston, USA) for the ceramide transporter protein (CERT), Good- pasture-antigen-binding protein (GPBP), LAG1 homolog ceramide synthase 1 (Lass1), neutral sphingomyelinase (nSMase), acid sphingomyelinase (aSMase), and sphingomyelin synthase 1 (SMS1); BAX, BAD, Bcl-2 and Bcl-XL. All samples were analyzed in duplo using the LightCycler 480 SYBR Green I Master kit (Roche, Almere, the Netherlands). Samples negative for RevertAid Reverse Transcriptase were used as appropriate control to ensure specific amplification. The real-time PCR was performed on a LightCycler 480 system (Roche Applied Science, Almere, the Netherlands). To standardize for the amount of cDNA, the geomean of three reference genes $\beta$-actin, hypoxyxanthine-guanine phosphoribosyltransferase (HPRT) and glyceraldehyde 3-phosphate dehydrogenase (GAPDH) was used. Quantification cycle values were extracted with the Lightcycler 480 software (Conversion LC 480 and LingRegPCR version 9.19beta) and calculated based on the cycle threshold $(\mathrm{Ct})$ values.

\section{Western blotting}

Frozen brains were homogenized in CelLytic MT Mammalian Tissue Lysis/Extraction Reagent (Sigma-Aldrich, St-Louis, MO). The samples were then centrifuged at $15000 \mathrm{~g}$ for $10 \mathrm{~min}$. The protein-containing supernatants were collected and the concentration of total protein was measured by Bradford, using bovine serum albumin (BSA) as a standard [134]. Equal amounts of total protein $(30 \mu \mathrm{g})$ from each sample were separated using 10\% SDS-PAGE gels and transferred onto a nitrocellulose membrane (Millipore, Amsterdam, the Netherlands). Blocking was 
performed with 5\% BSA. Next, the membranes were incubated overnight at $4{ }^{\circ} \mathrm{C}$ using polyclonal anti-GPBP/CERT (1:2500 dilution, epitope 1-50 of human GPBP, Bethyl Laboratories, Montgomery, TX). Membranes were then incubated with donkey anti-mouse IRDye700DX conjugated (1:10000 dilution, Rockland Immunochemicals, Gilbertsville). Mo-noclonal mouse anti-rabbit GAPDH (1:2000000 dilution, Fitzgerald Industries, Concord USA) was used as a loading control. Targeted proteins were analyzed using the LICOR Odyssey scanner (Li-Cor Bioscience, Lincoln, USA) and ImageJ software (National Institutes of Health, Bethesda, MD).

\section{Measurement of sphingolipids}

Brain pieces $(50 \mathrm{mg}$ ) were homogenized using a Qiagen bead beater for $10 \mathrm{~min}$ at $30 \mathrm{~Hz}$ in an 80/15/5 (v/v/v) mix of methanol, acetonitrile and HPLC grade water yielding a $25 \mathrm{mg} / \mathrm{ml}$ tissue homogenate. The samples were sonicated for $10 \mathrm{~min}$ and then clarified by centrifugation for $5 \mathrm{~min}$ at $1500 \mathrm{~g}$. The samples were then diluted 1:10 with the homogenization solvent yielding a final concentration of 2.5 $\mathrm{mg}$ tissue/ml. Glycosphingolipid content was determined as previously described [135] with slight modifications. Briefly, $75 \mu \mathrm{L}$ of brain homogenate was extracted with $600 \mu \mathrm{L}$ of $\mathrm{CHCl} 3 / \mathrm{MeOH} 1 / 2$ ( $\mathrm{vol} / \mathrm{vol})$. The extract was centrifuged for $10 \mathrm{~min}$ at $13200 \mathrm{rpm}$ and the pellet discarded. $460 \mu \mathrm{L}$ of CHCl3/MQ-H2O 1/1.3 (vol/vol) was added, mixed, and centrifuged for $3 \mathrm{~min}$ at $13200 \mathrm{rpm}$ to separate the phases. The lower phase was collected and the upper phase re-extracted with $400 \mu \mathrm{L}$ of $\mathrm{CHCl3}$. The combined lower phases were dried under $\mathrm{N} 2$ flow, taken up in 500 $\mu \mathrm{L}$ of freshly prepared $0.1 \mathrm{M} \mathrm{NaOH}$ in $\mathrm{MeOH}$, and deacetylated in a microwave (SAM-155, CEM corp.) for $60 \mathrm{~min} .100 \mu \mathrm{L}$ of this solution was derivatized with $50 \mu \mathrm{L}$ o-phtaldehyde (OPA) reagent. The OPA-derivatized lipids were separated by HPLC and quantified as previously described, using C17 sphinganine as an Internal Standard (Avanti Polar Lipids, Alabama, USA) [135].

\section{Flow cytometric analysis}

Total brains were placed in ice-cold plating medium consisting of DMEM supplemented with $10 \%$ fetal bovine serum (FBS), penicilline/streptavidine and glu- 
tamate. The tissue was mechanically disrupted using a glass homogenizer. The cell suspension was centrifuged and the pellet was resuspended in $5 \mathrm{ml}$ of plating medium. The crude cell suspension was then passed through a $100 \mu \mathrm{m}$ nylon cell strainer to remove large cell clumps. Next, cell numbers were counted using a Bürker's chamber and divided into different Eppendorf tubes (106 cells $/ 0.5 \mathrm{ml}$ per tube). The viability of the cells was monitored using trypan blue.

Apoptotic and necrotic cells were detected with AnnexinV conjugated to FITC and propidium iodide. The staining was executed according to the manufacturer's instructions (AnnexinV-FITC Apoptosis Dectection Kit, BD Biosciences Pharmingen, Breda, the Netherlands). To identify different cell types, extracellular staining for OX42 (microglia) and intracellular staining for GFAP (astroglia), CNPase (oligodendrocytes) and NF-200 (neurons) were performed. Shortly, the cells were washed with staining buffer [phosphate buffered saline (PBS) $+2 \%$ fetal bovine serum (FBS)]. After washing, cells were incubated for $30 \mathrm{~min}$ at RT with the primary antibody: mouse anti-rat CD11b/c (clone OX42, BD pharmingen, 1:100 dilution). The cells were washed twice followed by incubation with the secondary antibody (donkey anti-mouse alexa 488, 1:100 dilution). For intracellular staining, cells were fixed with $4 \%$ paraformaldehyde in staining buffer for $20 \mathrm{~min}$ at RT. Permeabilization was done after two more washing steps with permeabilization buffer $(0.05 \%$ saponin $+2 \%$ FBS + PBS $)$ for 10 min at RT. The cell suspension was then incubated with the primary antibody for 30 or 45 minutes at RT (mouse anti-ovine CNPase (Clone 11-5B), Sigma-Aldrich, 1:200 dilution; rabbit anti-ovine GFAP (DAKO), 1:200 dilution; mouse anti-NF-200 (Abcam, 1:100 dilution). After two washing steps, the cell suspension was incubated with the secondary antibody (donkey anti-mouse or donkey anti-rabbit alexa 488, 1:100 dilution) for $30 \mathrm{~min}$ at RT. Finally, all samples were washed and kept at $4{ }^{\circ} \mathrm{C}$ in the dark until analysis. All samples were measured on a FACScalibur flow cytometry system (BD Biosciences, Breda, the Netherlands) equipped with an argon ion laser (488nm). Analysis was done using the Cell Quest Pro software (BD Biosciences). Forward and sideward light angle scatters were collected from all samples. Samples were gated (R1) to exclude cell debris and cellular aggregates for further analysis. For each marker, the mean fluorescence intensity and the percentage of positive cells stained above 
background were measured for a total of 10000 cells per sample within the gate (R1). The cut off was defined using control tissue negative for the different markers processed and stained alongside the experimental samples. The mean fluorescence intensity was corrected for autofluorescence using the values from cells only incubated with secondary antibody.

\section{Statistical analysis}

All results were expressed as mean + standard error of the means (SEM). Statistical analyses were conducted using standard statistical software (SPSS 17.0 software). Normality was tested using the Kolmogorov-Smirnov test. All results were normally distributed. Comparisons between control and FA animals were analyzed using Student's t-test. $\mathrm{P}<0.05$ was considered statistically significant.

\section{RESULTS}

Pilot data have shown that a global FA preconditioning stimulus lowers the amount of apoptotic cell death in PA animals [28]. Since ceramide is an important regulator of cell growth and apoptosis [46], the involvement of several components of the ceramide signaling pathway (Figure 1) in endogenous neuroprotection was studied at several time points after sublethal FA (Figure 2B).

\section{Changes in mRNA levels of ceramide metabolism key enzymes at $6 \mathrm{~h}$ and $12 \mathrm{~h}$ after $\mathrm{FA}$}

To investigate whether ceramide metabolites change at $6 \mathrm{~h}$ and $12 \mathrm{~h}$ after FA preconditioning, we examined by RT-PCR the expression of four enzymes involved in ceramide metabolism: Lass1, SMS1, aSMase and nSMase [47]. mRNA levels of Lass1, the enzyme which converts sphinganine to ceramide [136], were increased 6 $\mathrm{h}$ after FA compared with respective controls (Figure 3A). mRNA levels of SMS1, the enzyme which catalyzes the formation of sphingomyelin from ceramide [47], were also increased $6 \mathrm{~h}$ after FA (Figure 3B) indicating that upregulation of ceramide synthesis is accompanied by increased levels of sphingomyelin [47]. No changes were seen in mRNA levels of nSMase (data not shown), the membrane 
bound enzyme which breaks down sphingomyelin giving rise to ceramide [48]. However, aSMase levels were found to be increased $12 \mathrm{~h}$ after FA compared with control levels (Figure 3C). ASMase is localized in lysosomes and regulates cellular membrane turnover [48].

Once generated in the ER, ceramide must reach the Golgi apparatus in order to be converted to more complex sphingolipids [48]. CERT/GPBP is the protein responsible for the transport of ceramide from the ER to the Golgi for its subsequent transformation to sphingomyelin [48]. Both $6 \mathrm{~h}$ and $12 \mathrm{~h}$ after FA lower CERT/GPBP protein levels were observed (Figure 3D), indicating that increased level of ceramide and sphingomyelin producing enzymes is counterbalanced by a reduction in the levels of the protein that delivers ceramide to form sphingomyelin.

The effect of enzymatic mRNA changes on sphingolipid levels was tested using HPLC. As expected, ceramide and sphingomyelin levels are unaffected (data not shown) in the early phase characterized mainly by alteration in enzymatic transcription levels. Surprisingly, the conversion of ceramide to galactosylceramide (GalCer) and glucosylceramide (GlcCer) was inhibited $12 \mathrm{~h}$ after FA (Figure 3E), which paralleled the increase in aSMase mRNA levels (Figure 3C).

\section{Sphingolipid levels are increased $96 \mathrm{~h}$ after FA}

Ceramide, sphingomyelin and GalCer+GlcCer levels were found to be upregulated $96 \mathrm{~h}$ after FA compared with control levels (Figure 4A-C). This peak may be due to the early increased expression of the aforementioned enzymes. Also, higher aSMase mRNA levels (Figure 4D) were maintained during this later phase, suggesting the lysosomal catabolism of sphingomyelin and corresponding ceramide formation $[47,48]$. Although no significant increase in GPBP/CERT protein levels could be observed (Figure 4F), higher CERT mRNA levels were seen $96 \mathrm{~h}$ after FA (Figure 4E), indicating that the protein is now active and delivering ceramide to the Golgi to produce sphingomyelin. This is consistent with the high levels of ceramide, sphingomyelin and glycosphingolipid that persist in this phase. These data provide supporting evidence for a possible role of the ceramide signaling pathway in the induction of neuroprotection as sphingolipid levels are highly modulated at the time of birth, when there is a high risk of severe PA. 
A

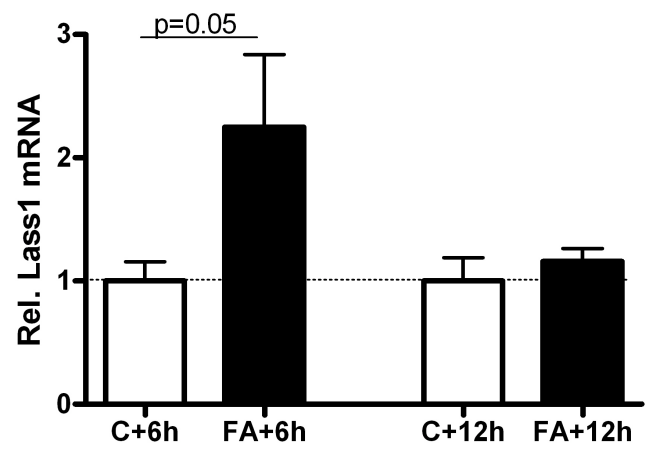

C

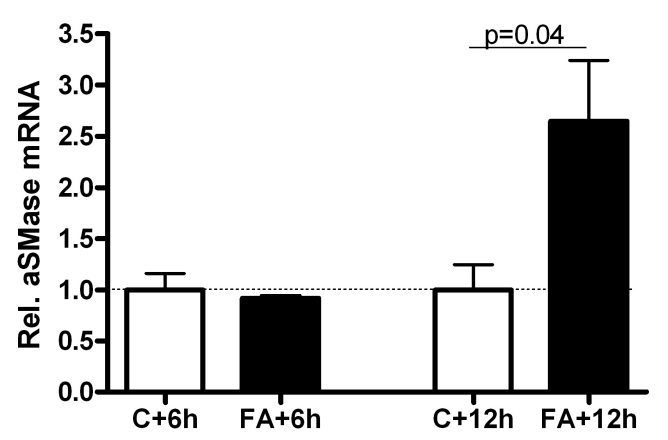

E

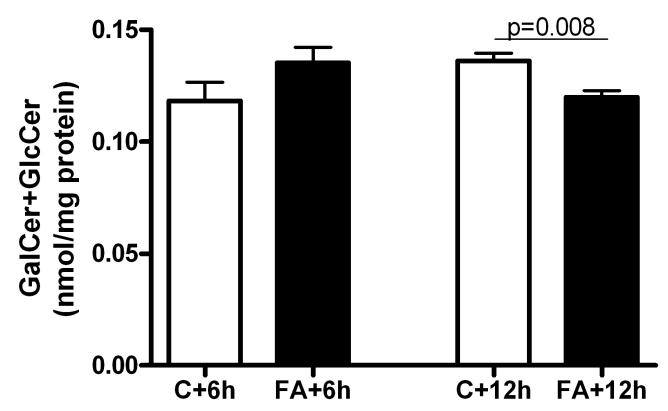

B

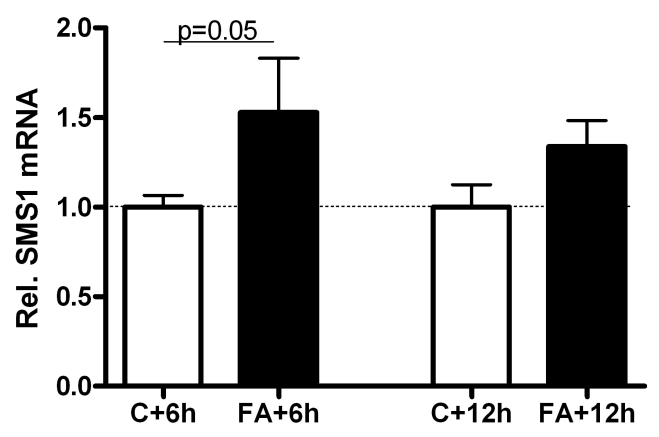

D

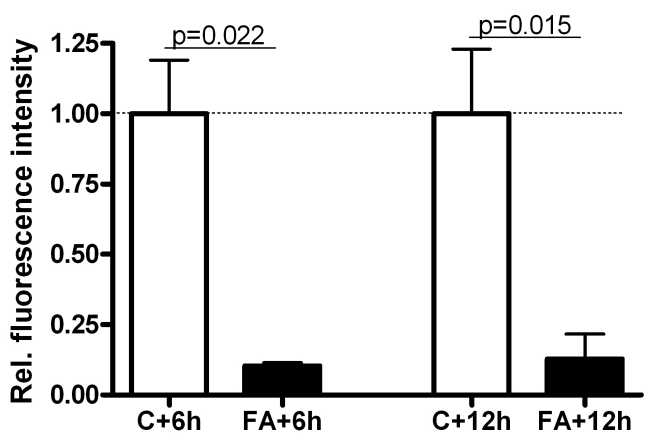

Figure 3: Changes in mRNA levels of ceramide metabolism key enzymes at $6 \mathrm{~h}$ and $12 \mathrm{~h}$ after FA. Prenatal relative mRNA levels of Lass1 (A), SMS1 (B) and aSMase (C) in control and FA animals. mRNA levels are relative to the geomean of measured housekeeping genes and normalized to control levels. Relative protein levels of CERT/GPBP normalized to GAPDH as loading control $6 \mathrm{~h}$ and $12 \mathrm{~h}$ after FA with corresponding control levels (D). GalCer+GlcCer concentration expressed in $\mathrm{nmol} / \mathrm{mg}$ protein in control and FA animals (E). Data shown as mean + SEM. 
A

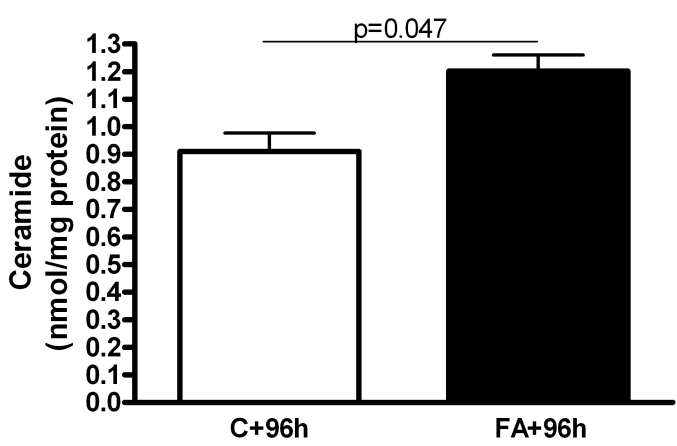

C

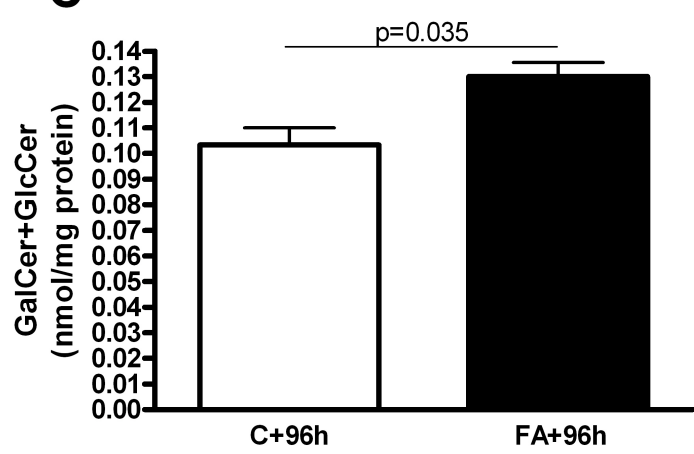

E

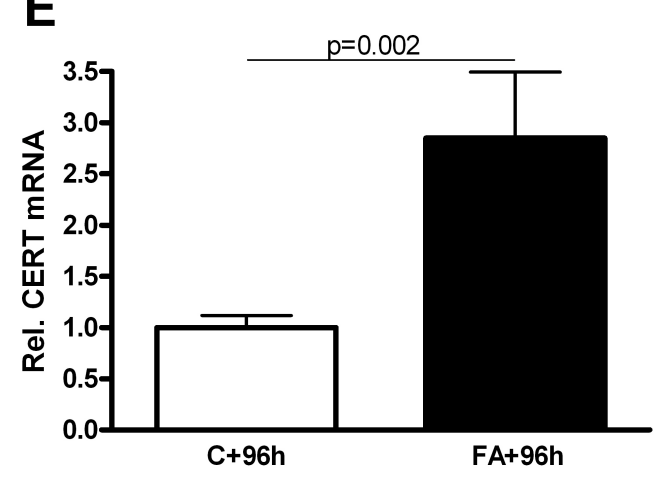

B

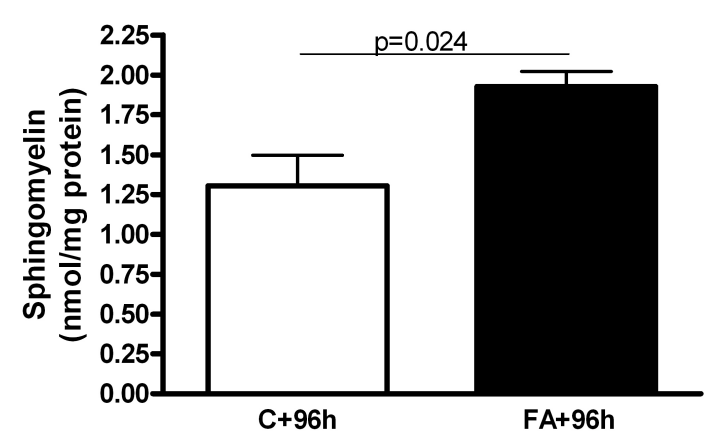

D

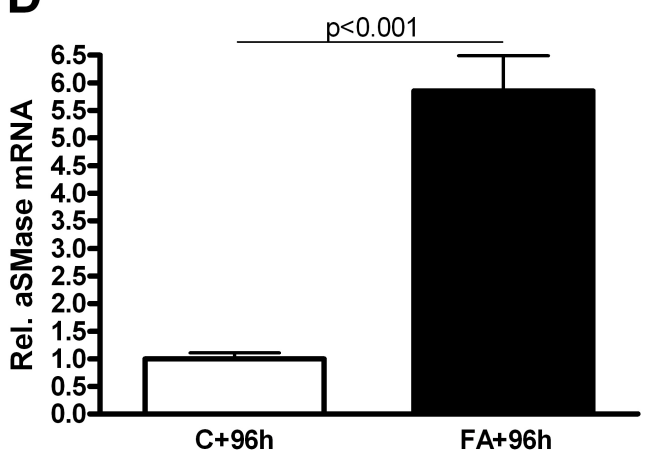

$\mathbf{F}$

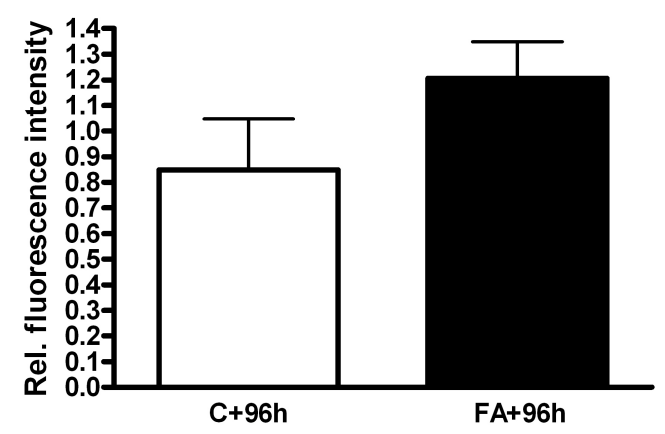

Figure 4: Sphingolipid levels are increased $96 \mathrm{~h}$ after FA. Ceramide (A), sphingomyelin (B) and GalCer+GlcCer (C) concentrations expressed in $\mathrm{nmol} / \mathrm{mg}$ protein in control and FA animals. Relative mRNA levels of aSMase (D) and CERT (E) in control and FA animals. mRNA levels are relative to the geomean of measured housekeeping genes and normalized to control levels. Relative protein levels of CERT/GPBP normalized to GAPDH as loading control $96 \mathrm{~h}$ after FA with corresponding control levels $(\mathrm{F})$. Data shown as mean + SEM. 


\section{Increased sphingolipid levels are preceded by induction of apoptosis and inflammation}

Growing evidence indicates a signaling role for ceramide in asphyxia-induced apoptosis $[55,137]$. To determine the effects of the sphingolipid changes on apoptotic levels, we quantified the amount of live, apoptotic and necrotic cells in total brain of control versus FA animals by FACS analysis. Figure 5A shows that although almost $35 \%$ of the cells undergo developmental apoptosis, the amount of live cells decreased after FA due to increased apoptosis but not necrosis and that neurons contribute most to this increase in apoptosis (Figure 5B). Moreover, the asphyctic brain seemed to generate an inflammatory response by increasing the amount of astrocytes and microglia (Figure 5B). Data obtained by RT-PCR were in line with the above results. We primarily focused on the Bcl-2 family of pro- and antiapoptotic genes since these represent effectors of the ceramide-mediated apoptotic pathway [137]. mRNA levels of all tested genes were upregulated $96 \mathrm{~h}$ after FA compared with control levels (Figure 5C).

In conclusion, FA induces increased sphingolipid levels which are correlated with higher levels of apoptotic neurons and activated astrocytes and microglia.

\section{Increased sphingolipid levels are sustained till seven days after birth and correlated with increased GPBP/CERT protein levels}

To investigate whether the changes in ceramide metabolism after sublethal FA would persist after birth, we assessed ceramide synthesis $2 \mathrm{~h}, 6 \mathrm{~h}$ and $7 \mathrm{~d}$ after birth (Figure 2B). At all postnatal time points, ceramide levels remained increased (Figure 6A). This upregulation in ceramide levels was correlated with a persistent increase in mRNA levels of aSMase (Figure 6C). $6 \mathrm{~h}$ and $7 \mathrm{~d}$ after birth, also increased levels of sphingomyelin were seen (Figure 6B). We could detect a significant upregulation of GPBP/CERT protein levels at 7 days after birth (Figure 6D). Although we could not find differences in mRNA levels for the measured apoptotic genes (data not shown), we previously showed that apoptotic levels are highest 7 days after birth in PA animals (pilot data, [28]). However, when animals experienced both fetal and perinatal asphyxia, apoptosis levels tended to be normal [28]. 
A

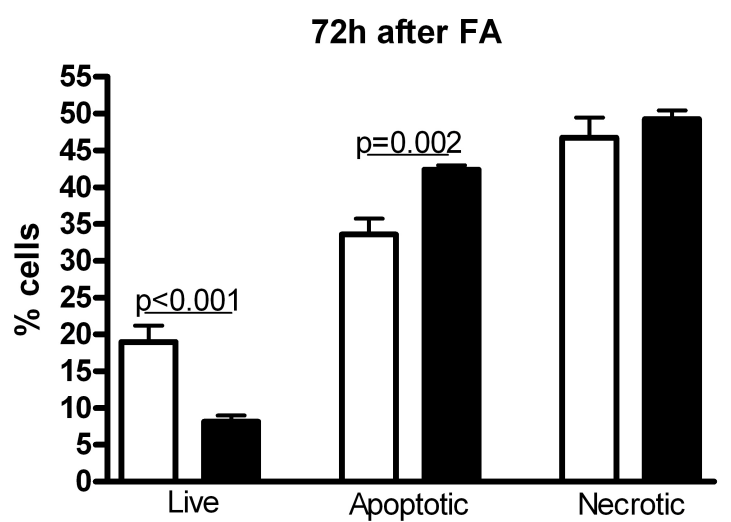

B

72h after FA
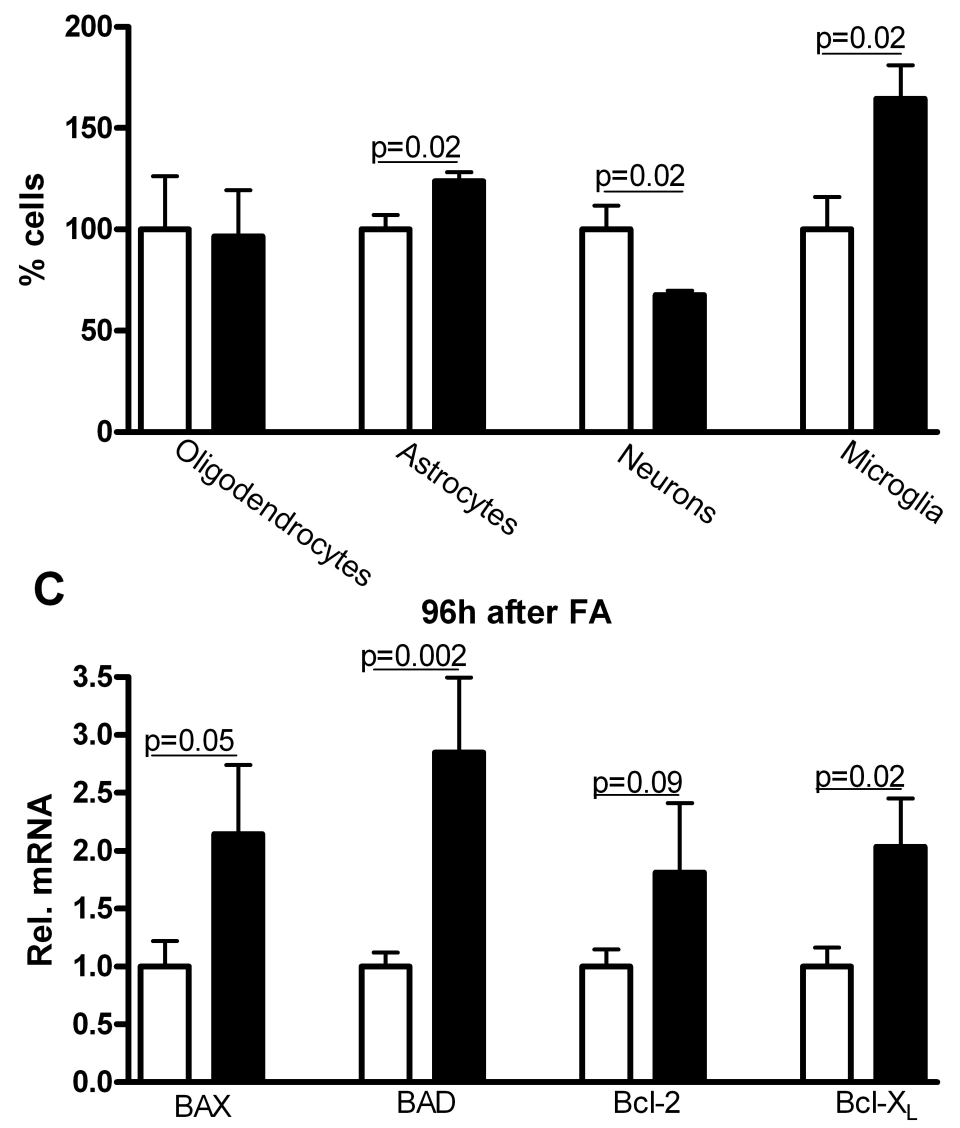

Figure 5: Increased sphingolipid levels are preceded by induction of apoptosis and inflammation. Amount of live, apoptotic and necrotic cells (A) and amount of specific cell types (B) $72 \mathrm{~h}$ after FA compared with respective control levels. Relative mRNA levels of BAX, BAD, Bcl-2 and Bcl-XL $96 \mathrm{~h}$ after FA (C). mRNA levels are relative to the geomean of measured housekeeping genes and normalized to control levels. Data shown as mean + SEM. 
These results suggest that FA induces important changes in ceramide and sphingomyelin levels that are sustained even 7 days after birth providing neuroprotection against PA-induced apoptosis. In addition, an important role of the enzyme aSMase and the GPBP/CERT mediated transport should be considered when investigating the preconditioning effect of FA.

A

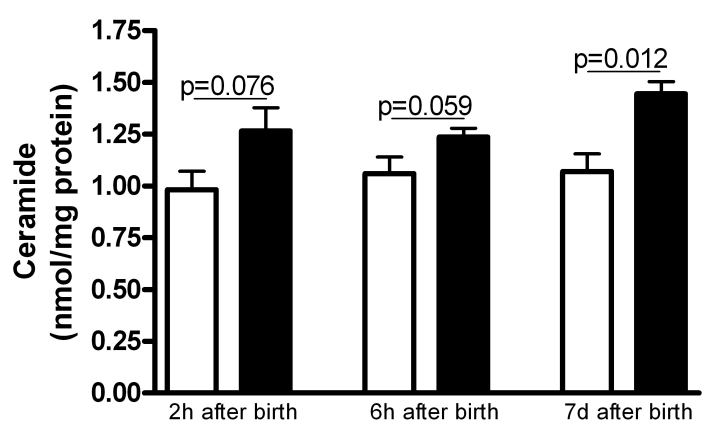

C

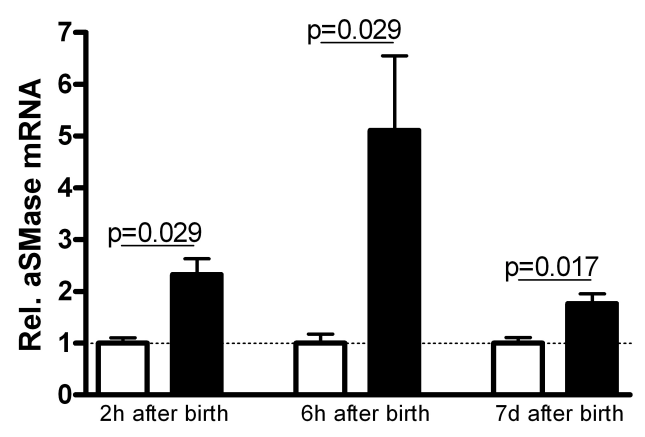

B

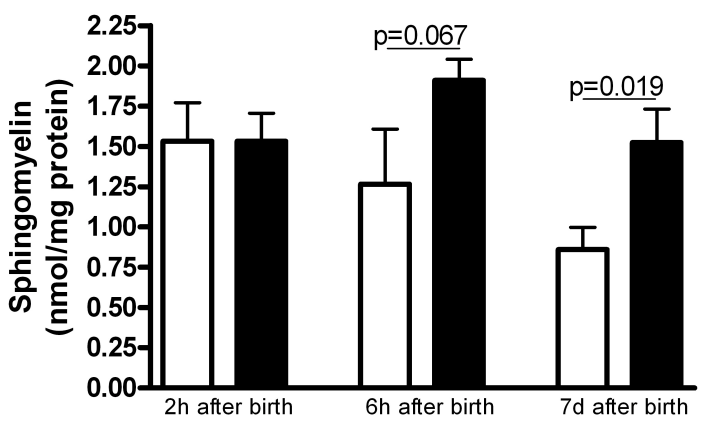

D

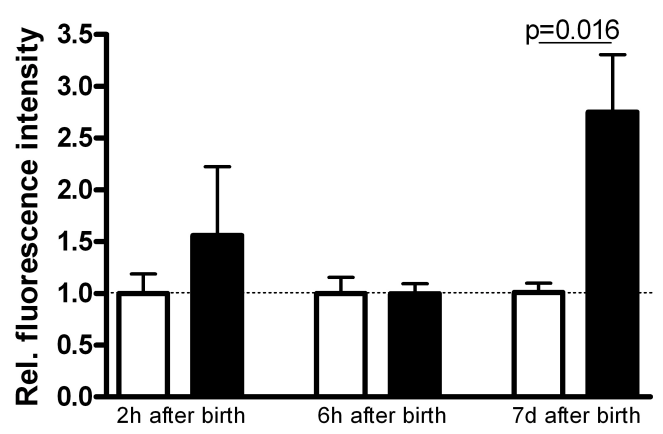

Figure 6: Increased sphingolipid levels are sustained till seven days after birth and correlated with increased GPBP/CERT protein levels. Ceramide (A) and sphingomyelin (B) concentrations expressed in $\mathrm{nmol} / \mathrm{mg}$ protein in control (white bars) and FA (black bars) animals. mRNA levels of aSMase (C) are presented relative to the geomean of measured housekeeping genes. Relative protein levels of CERT/GPBP normalized to GAPDH as loading control in control (white bars) and FA (black bars) animals (D). Data shown as mean + SEM. 


\section{DISCUSSION}

Our results show a biphasic response to FA starting with an acute increase in mRNA levels of the enzymes Lass1 and SMS1. Later, aSMase, ceramide, sphingomyelin, galactosyl- and glycosylsylceramide levels were increased together with levels of apoptosis and inflammatory cells. Even after birth, ceramide and sphingomyelin levels remained upregulated in FA subjected animals. Together, these data demonstrate that FA induces both acute and long lasting changes in ceramide metabolism. Our data also indicate that ceramide metabolism is an important factor which may function in concert with inflammatory and apoptotic factors in our global asphyxia model of the fetal/neonatal rat.

Many studies focus on the induction of tolerance to hypoxic-ischemic insults. Nevertheless, the mechanism by which a period of cerebral asphyxia, insufficient to produce brain damage, can provide neuroprotection against a subsequent more severe insult is not completely understood. Injury after an asphyctic insult has been shown to primarily occur by apoptosis in the immature brain [137]. In our rat model of global asphyxia, we have shown that apoptotic cell numbers are higher in prefrontal cortex and striatum of asphyctic brains [28]. When animals experienced fetal asphyxia four days before the severe asphyctic insult at birth, apoptotic cell numbers were equal to control animals [28]. In the present study, we investigated the role of ceramide signaling in FA-induced neuroprotection since biological effects of ceramide include the regulation of inflammation and, more importantly, apoptosis [138]. Previous studies have shown that ceramide levels in the brain increase both after acute and chronic ischemia [55,132]. We found two separate phases in the ceramide response to FA; an early response with increased mRNA levels of the enzymes Lass1 and SMS1 and a late response with increased levels of aSMase, CERT/GPBP, ceramide and sphingomyelin. The acute effect of FA is characterized by increased levels of the key enzymes of ceramide metabolism. Lass proteins regulate de novo synthesis of ceramide and their expression is induced after brief exposure to hypoxia and oxygen/glucose deprivation [47]. Within a few hours of induced Lass 1 expression, increased levels of ceramide, sphingomyelin and gly- 
cosphingolipid could be detected [136]. Increased levels of acid (but not neutral) SMase were observed $12 \mathrm{~h}$ after FA. The enzyme aSMase has been shown to be activated by many forms of stress and to participate in receptor signaling within minutes after stimulation [48]. Moreover, aSMase activation appears to mediate signaling pathways that induce programmed cell death [48]. Studies have shown that ischemia-induced ceramide levels require the activity of this enzyme [54,132]. Accordingly, the higher sphingolipid levels observed at $96 \mathrm{~h}$ after FA are due to the activation of two pathways, the de novo pathway (activated at $6 \mathrm{~h}$ after FA) and the catabolic pathway mediated by increased levels of aSMase.

The two phases in ceramide response were also seen in the expression pattern of the ceramide transporter CERT/GPBP. Stimulation of the de novo pathway is accompanied by a sharp decrease in CERT/GPBP protein levels. This is possibly as a mechanism to reduce the amount of ceramide that is converted to SM, thereby blocking the production of proapoptotic ceramides at the plasma membrane by aSMase. Interestingly, at $96 \mathrm{~h}$ after FA, CERT levels are increased together with an increase in ceramide and sphingomyelin. We speculate that this is a result of the induced inflammatory response $72 \mathrm{~h}$ post FA as inflammation and in particular cytokines are associated with increased CERT/GPBP-mediated transport [48]. Together with this increase in CERT/GPBP levels, inflammation and sphingolipid levels, we found higher levels of apoptotic neurons. Apoptosis is a common response to neuronal injury, especially during the neonatal period [137]. The Bcl-2 familiy proteins have been of focus in our research since they have been shown to act as downstream mediators of ceramide [139]. These proteins have opposing effects on the regulation of apoptosis with Bcl-2 and Bcl-XL functioning as apoptosis inhibitors and BAX and BAD promoting apoptosis [140]. In the current study, it seems that ceramide increases the expression of Bcl-2, Bcl-XL, BAX and BAD. Chen et al. demonstrated that upregulation of Bcl-2 and Bcl-XL are responsible for ceramide-induced neuroprotection [137]. This raises an important issue, indicating a dual role of ceramide ranging from protective to apoptotic. Studies have indicated a detrimental role for ceramide and shown that inhibiting ceramide might protect against hypoxic-ischemic neuronal injury [54, 132]. However, it has also been shown that ceramide can protect hippocampal and cortical neurons against in- 
jury, apoptosis and free radical forms $[55,137]$ and can mediate hypoxia tolerance induced by hypoxic preconditioning [58]. Specifically, it was shown that hypoxic preconditioning of neuronal cells produced a peak in intracellular ceramide levels $72 \mathrm{~h}$ post FA and provided resistance to subsequent severe hypoxia [58]. This protective effect is only observed when the timing of the second hypoxia stimulus coincided with increased ceramide release [141]. Contrary to the findings of Ginis et al. [141], we observed increased levels of apoptosis and induction of apoptotic genes in response to high levels of ceramide. This may be dependent on the age of the animals which differed between the studies. We did not investigate how two subsequent asphyctic insults affect the ceramide levels. However, we have shown that the apoptotic cell numbers are not altered when animals experience FA before to severe asphyctic insult [28]. Interestingly, at seven days after FA, the increased ceramide and sphingomyelin levels were correlated with increased levels of the ceramide transporter as was also the case at $96 \mathrm{~h}$ after FA. This raises the possibility that CERT/GPBP is able to modulate the immune response to an asphyctic stimulus. However, more research is needed to fully characterize the precise role of this protein in asphyctic brain damage.

In summary, at $96 \mathrm{~h}$ post FA, we found induction of aSMase leading to the upregulation of ceramide. Although ceramide was converted to sphingomyelin and GalCer+GlcCer, apoptosis and inflammation were increased. Even after birth, ceramide levels were increased in animals that previously experienced FA. However, these postnatal changes were not associated with significant changes in apoptosis or inflammation. Follow-up studies should focus on the combination of FA with a subsequent severe asphyctic insult to highlight more insights into the ceramide changes involved in FA preconditioning. 



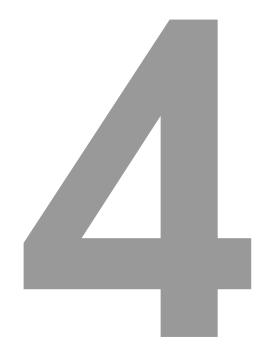

\section{The effects of fetal and perinatal asphyxia on neuronal cytokine levels and ceramide metabolism in adulthood}

Evi VLASSAKS, Antonio W.D. GAVILANES, Johan S. H. VLES, Sarah DEVILLE, Boris W. KRAMER, Eveline STRACKX and Pilar MARTINEZ-MARTINEZ

In press (Journal of Neuroimmunology) 


\section{ABSTRACT}

In a rat model of global fetal and perinatal asphyxia, we investigated if asphyxia and long-lasting brain tolerance to asphyxia (preconditioning) are mediated by modifications in inflammatory cytokines and ceramide metabolism genes in prefrontal cortex, hippocampus and caudate-putamen at the age of 8 months. Most significant changes were found in prefrontal cortex, with reduced LAG1 homolog ceramide synthase 1 expression after both types of asphyxia. Additionally, sphingosine kinase 1 was upregulated in those animals that experienced the combination of fetal and perinatal asphyxia (preconditioning), suggesting increased cell proliferation.

While cytokine levels are normal, levels of ceramide genes were modulated both after fetal and perinatal asphyxia in the adult prefrontal cortex. 


\section{INTRODUCTION}

Perinatal asphyxia (PA) is one of the most common causes of neonatal morbidity and mortality [97, 142]. Because of the lack of effective therapies, besides hypothermia [19], there is a high need for new approaches to treat infants suffering from asphyxia. Previously, we showed in a rat model of global asphyxia that the combination of a mild fetal asphyctic (FA) insult and a subsequent severe PA insult (=FA-PA; preconditioning) provided long-lasting protection against neuronal deficits normally observed after PA alone [27]. Gaining more insights into these mechanisms leading to brain tolerance will help the development of new specific therapeutics.

Ceramides are bioactive lipids which have emerged as important regulators of cell activation with a wide spectrum of activities ranging from the control of cell growth to cell death [48] and have been shown to be upregulated in hypoxicischemic brain damage $[47,132]$. Besides the changes in lipid metabolism, asphyxia triggers an inflammatory response $[33,34,37,99]$. The cytokines produced in this inflammatory response are suggested to affect the lipid metabolism in the brain [45] and in particular tumor necrosis factor (TNF)- $\alpha$ is recognized as an important stimulus for ceramide generation [143]. TNF- $\alpha$ also activates the promoter region of ceramide transporter (CERT) and Goodpasture antigen-binding protein (GPBP) [48], suggesting the involvement of these ceramide transporters in asphyctic brain damage as well.

We hypothesized that long-lasting brain tolerance to asphyxia is partly mediated by modifications of genes involved in inflammation and ceramide metabolism. To study this, a validated rat model was used [27, 28]. The prefrontal cortex, hippocampus and caudate-putamen (cpu) were examined for long-term changes in cytokine levels and enzymes and transporters involved in ceramide metabolism at the middle age of 8 months. Most significant changes were observed in prefrontal cortex, suggesting long-lasting neuroprotection in FA-PA animals by promoting cell proliferation in the prefrontal cortex. 


\section{MATERIAL AND METHODS}

\section{Animals and tissue processing}

All experiments were approved by the Animal Ethics Board of Maastricht University on animal welfare according to Dutch governmental regulations. SpragueDawley rats (Charles River, Germany) were kept under standard laboratory conditions [144]. Exclusively male offspring were used within this study to exclude hormonal influences $[145,146]$.

FA and PA were induced as previously described [27]. In this study 4 experimental groups are included: Control animals, born by caesarean section (C-section) $(\mathrm{n}=7)$; FA animals, only subjected to FA at embryonic day 17 (E17) and born by C-section $(n=10)$; PA animals, born by $C$-section and subjected to PA $(n=9)$; FAPA animals, subjected to both asphyctic insults at E17 and at birth respectively $(n=9)$. At the middle age of 8 months, the animals were sacrificed and the brain regions prefrontal cortex, hippocampus and cpu were isolated. Regions of one hemisphere were snap-frozen in liquid nitrogen, regions of the other hemisphere were fixated with $4 \%$ paraformaldehyde, embedded in Tissue-Tek OCT Compound (Sakura, Alphen aan den Rijn, the Netherlands) and stored at $-80^{\circ} \mathrm{C}$ prior to further analysis.

\section{RNA isolation and cDNA synthesis}

Snap-frozen tissue samples were homogenized in Trizol Reagent (Invitrogen, Breda, the Netherlands), following manufacturer's protocol. cDNA was synthesized using RevertAid First Strand cDNA synthesis kit (Fermentas, St Leon Rot, Germany), according to manufacturer's guidelines. The samples were diluted 1 to 20 with diethyl pyrocarbonate (DEPC)-treated sterilized water and stored at $-80^{\circ} \mathrm{C}$ prior to analysis.

\section{Quantitative PCR}

Quantitative PCR (qPCR) was performed for each gene in duplicate using the LightCycler 480 SYBR Green I Master kit (Roche, Almere, the Netherlands), ac- 
cording to manufacturer's protocol. For each gene, mRNA from every single animal was run in duplicate. Primers (Table 1) were designed by Primer3plus software. Samples negative for RevertAid Reverse Transcriptase were used as controls to check for genomic contamination. The qPCR data was standardized to the geomean of three reference genes, $\beta$-actin, GAPDH and HPRT (Table 1), and finally normalized to control levels. Quantification cycle values were extracted with the Lightcycler 480 software (Conversion LC 480 and LingRegPCR version 9.19beta) and calculated based on the cycle threshold $(\mathrm{Ct})$ values.

Table 4.1: Primer sequences for the selected genes of interest for qPCR analysis

\begin{tabular}{lll}
\hline Gene & Forward sequence $\left(5^{\prime}-3^{\prime}\right)$ & Reverse sequence $\left(5^{\prime}-3^{\prime}\right)$ \\
\hline$\beta$-actin & TTGCTGACAGGATGCAGAAG & TGATCCACATCTGCTGGAAG \\
GAPDH & CTCCCATTCTTCACCTTTG & ATGTAGGCCATGAGGTCCAC \\
HPRT & TTGCTGGTGAAAAGGACCTC & TCCACTTTCGCTGATGACAC \\
IL-10 & CCTGCTCTTACTGGCTGGAG & TGTCCAGCTGGTCCTTCTT \\
IL-6 & AAAGCCAGAGTCATTCAGAGC & GAGCATTGGAAGTTGGGGTA \\
IL-1 $\beta$ & TACCTATGTCTTGCCCGTGGAG & ATCATCCCACGAGTCACAGAGG \\
TNF- $\alpha$ & TGCCTCAGCCTCTTCTCATT & GGGCTTGTCACTCGAGTTTT \\
CERT & ATAGAGGAACAGTCACAGAGTG & CTGTACCATCTCTTCAACCTTTTG \\
GPBP & ATGTCCACAGATTCAGCTCCC & CTTCTTCTACAACCAGCTGCC \\
Lass1 & CTTCTTCAACACTCTGCTGCTG & TCTTCCAGTTCACGCATCTCG \\
nSMase & TCAGGAAGACCCTTGCTCTG & GACAGCCCCAAAATCATCAC \\
SMS & GGTATCACACGATGGCCAAT & GATCGAGGTACAATCCCTTGG \\
SphK1 & AAACCCCTATGTAGCCTCCC & TAGTGACCTGCTCGTGCC \\
Sph1PP & CACGTTACCCTTAGCTATCCC & CAACATCCCTATGACGACCC \\
\hline
\end{tabular}

\section{Protein extraction}

Proteins were extracted from tissue embedded in Tissue-Tek OCT Compound using QProteome FFPE Tissue Kit (Qiagen, Venlo, the Netherlands), according to the manufacturer's protocol. The protein concentration was determined using the Bicinchoninic acid (BCA) method (Thermo Scientific, Landsmeer, the Netherlands) also according to manufacturer's guidelines. 


\section{Western blotting}

Equal amounts of total protein $(30 \mu \mathrm{g})$ were separated using 12\% SDS-PAGE gels and transferred onto a nitrocellulose membrane (Millipore, Amsterdam, the Netherlands). Blocking was performed with 5\% Bovine Serum Albumin (BSA) and membranes were incubated overnight at $4{ }^{\circ} \mathrm{C}$ with mouse anti-TNF- $\alpha$ (1:500 dilution, Santa Cruz Biotechnology, Santa Cruz, USA) and rabbit anti-CERT (1:2500 dilution; epitope 300-350 of human GPBP, Bethyl Laboratories, Montgomery, TX, USA). After washing, membranes were incubated for $1 \mathrm{~h}$ at room temperature with donkey anti-mouse and goat anti-rabbit (1:10,000 dilution, Rockland Immunochemicals, Gilbertsville, USA). GAPDH was detected with mouse anti-rabbit GAPDH (1:2,000,000 dilution, Fitzgerald Industries, Concord, USA) as loading control. Finally, membranes were scanned using the Odyssey infrared imaging system (Li-Cor Bioscience, Lincoln, USA).

\section{Statistical analysis}

All results are expressed as mean + standard error of the means (SEM). Statistical analyses were conducted using standard statistical software (SPSS 18.03 software). Normality was tested using the Kolmogorov-Smirnov test. All results were normally distributed. Comparisons were tested with one-way analysis of variance (ANOVA). Significant effects were further analyzed by post-hoc least significant difference (LSD) test corrections. $\mathrm{p}<0.05$ was considered statistically significant.

\section{RESULTS}

To determine whether FA and/or PA induced chronic changes in genes involved in inflammation and the ceramide pathway, qPCR and Western blot techniques were performed on the prefrontal cortex, hippocampus and cpu (Table 2). A regionspecific response after asphyxia was observed with no changes in cpu, minor hippocampal differences in TNF- $\alpha$ and CERT mRNA (data not shown) and most significant changes in the prefrontal cortex. 
Prefrontal cortical mRNA (Figure 1A-C) and protein analysis (Figure 2A-B) showed no significant effects between groups for TNF- $\alpha$ and the ceramide transporters CERT and GPBP. However, mRNA levels of enzymes involved in ceramide metabolism revealed chronic changes. LAG1 homolog ceramide synthase 1 (Lass1) expression was significantly reduced in the FA $(\mathrm{p}=0.047)$ and PA $(\mathrm{p}=0.05)$ group compared to controls. When animals were subjected to both asphyctic insults (FAPA group), Lass1 levels were comparable with controls and significantly upregulated compared to the FA or PA animals ( $\mathrm{p}=0.018$ and $\mathrm{p}=0.045$ respectively) (Figure 1D). Sphingosine Kinase (SphK) 1 was upregulated in the FA-PA condition compared to controls and FA animals ( $\mathrm{p}=0.039$ and $\mathrm{p}=0.019$ respectively) and there was a clear elevated trend compared to the PA group ( $\mathrm{p}=0.071$ ) (Figure 1G). Neutral sphingomyelinase (nSMase), sphingomyelin synthase (SMS) and sphingosine1-phosphate phosphatase (Sph1PP) mRNA levels were not affected by any of the asphyctic insults (Figure 1E, F, H).

Table 4.2: Overview of analyzed parameters with respective techniques in prefrontal cortex, hippocampus and caudate-putamen (cpu).

\begin{tabular}{llll}
\hline Parameter & Prefrontal cortex & Hippocampus & CPU \\
\hline IL-10 & Q-PCR & Q-PCR & Q-PCR \\
IL-6 & Q-PCR & Q-PCR & Q-PCR \\
IL-1 $\beta$ & Q-PCR & Q-PCR & Q-PCR \\
TNF- $\alpha$ & Q-PCR, WB & Q-PCR, WB & Q-PCR \\
CERT & Q-PCR, WB & Q-PCR, WB & Q-PCR \\
GPBP & Q-PCR, WB & Q-PCR, WB & Q-PCR \\
Lass1 & Q-PCR & Q-PCR & Q-PCR \\
nSMase & Q-PCR & Q-PCR & Q-PCR \\
SMS & Q-PCR & Q-PCR & Q-PCR \\
SphK1 & Q-PCR & Q-PCR & Q-PCR \\
Sph1PP & Q-PCR & Q-PCR & Q-PCR \\
\hline
\end{tabular}


A

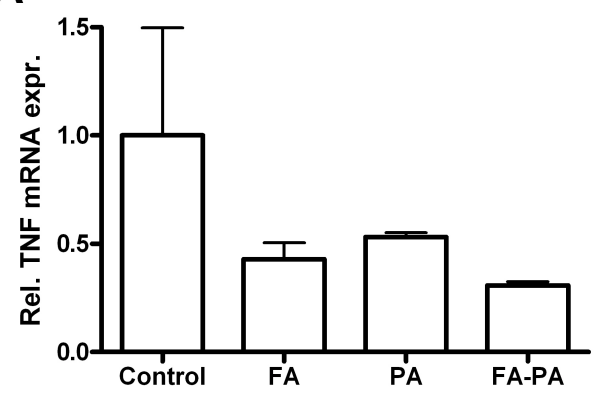

C

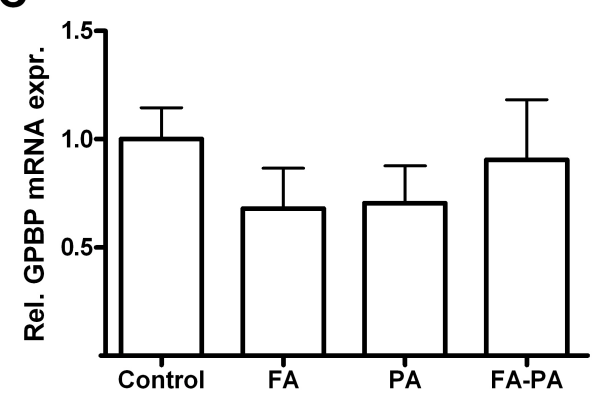

$\mathbf{E}$
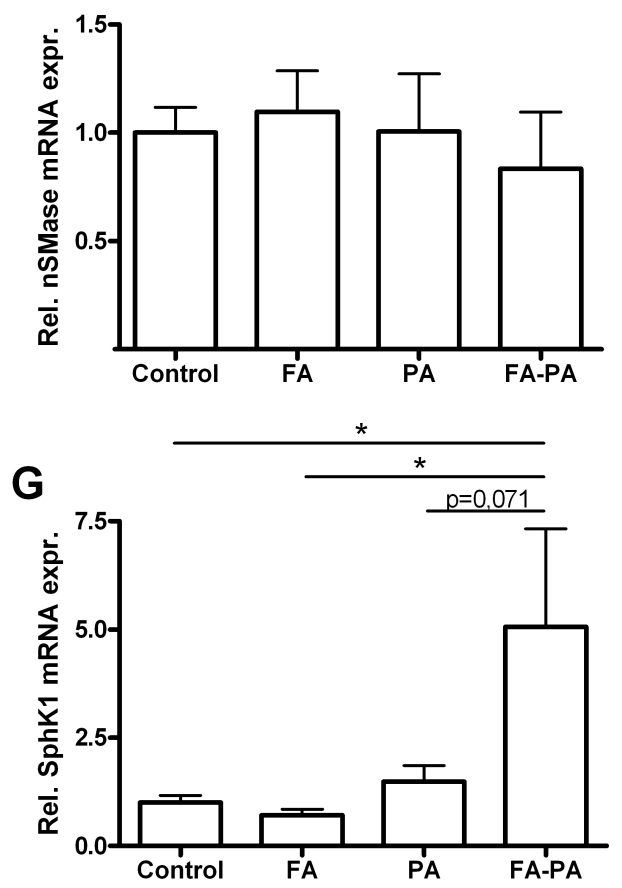

B

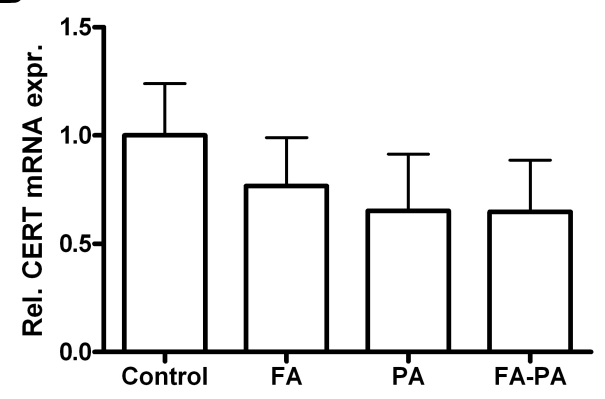

D

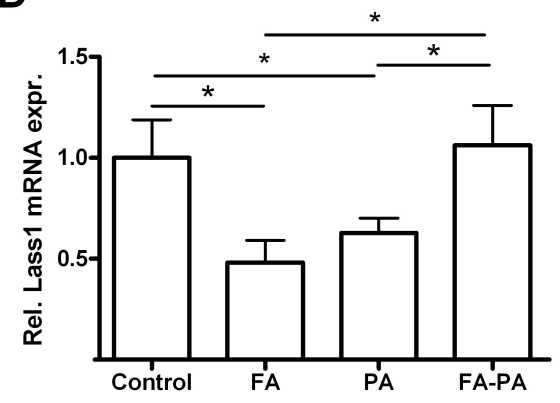

$\mathbf{F}$

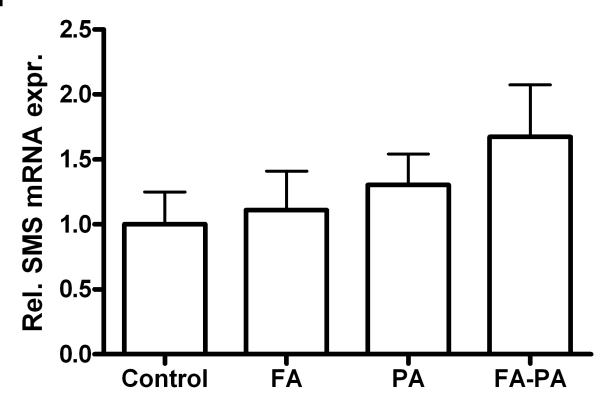

H

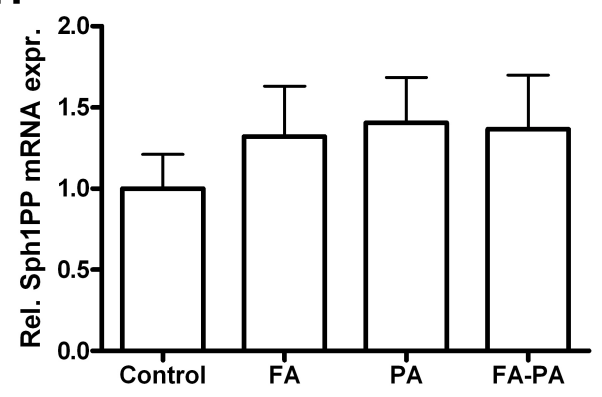

Figure 1: Relative prefrontal cortical mRNA levels. Bar graphs depict relative mRNA levels in prefrontal cortex of tumor necrosis factor (TNF)$\alpha(\mathrm{A})$, ceramide transporter protein (CERT) (B), goodpasture-antigenbinding protein (GPBP) (C), LAG1 homolog ceramide synthase 1 (Lass1) (D), neutral sphingomyelinase (nSMase) (E), sphingomyelin synthase 1 (SMS) (F), sphingosine kinase 1 (SphK1) (G) and sphingosine-1phosphate phosphatase (Sph1PP) (H). ${ }^{*} \mathrm{p}<0.05$. 
A

TNF- $\alpha$

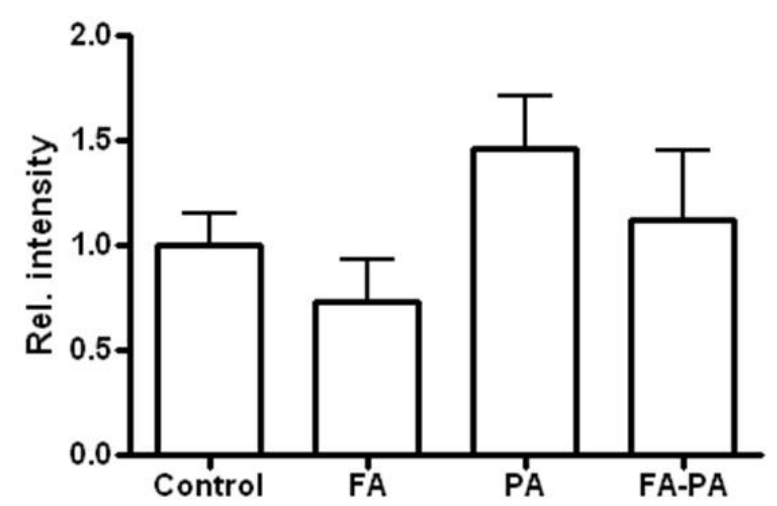

B

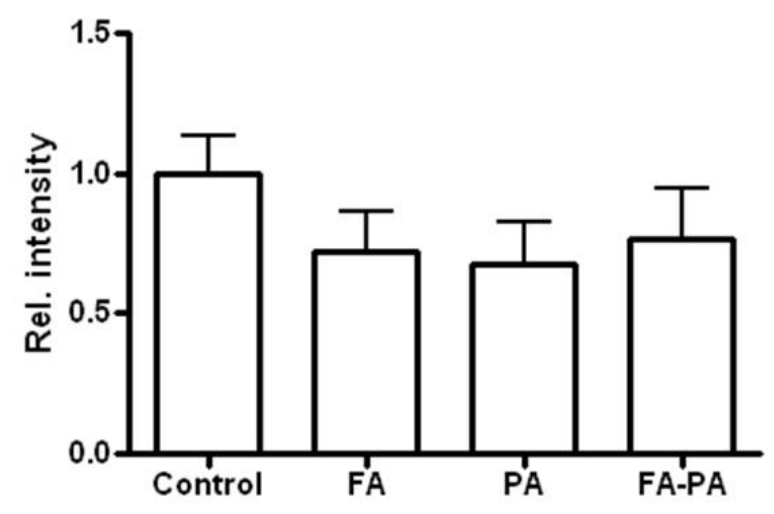

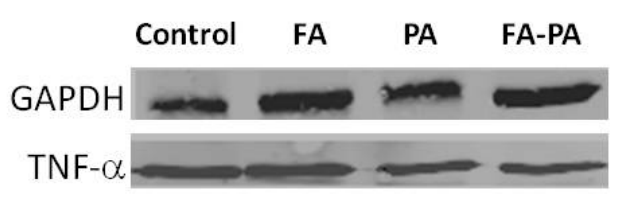

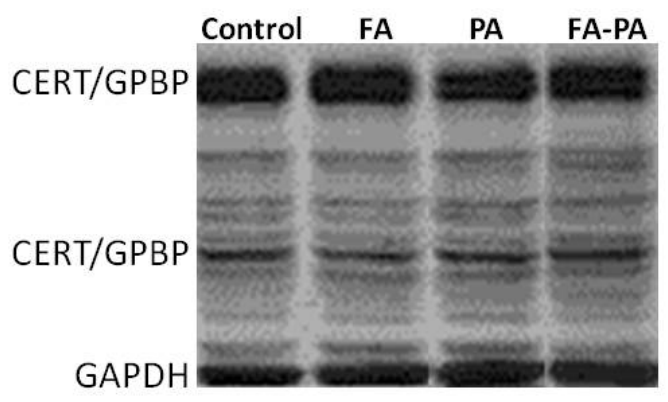

Figure 2: Relative protein levels of TNF- $\alpha$ and CERT/GPBP in prefrontal cortex. Bar graphs represent relative band intensity of TNF- $\alpha$ (A) and CERT/GPBP (B) with GAPDH as loading control (left). Representative Western Blots are depicted at the right side, showing TNF- $\alpha$ (A) and CERT/GPBP (B) in Control, FA, PA and FA-PA animals.

In summary, these results indicate that Lass 1 levels decreased after asphyxia irrespective the time point and severity of the insult. However, when both asphyctic insults are combined (FA-PA), Lass1 levels were comparable to normal. Additionally, in these FA-PA animals SphK1 mRNA was highly expressed. 


\section{DISCUSSION}

We aimed to determine the long-term effects of FA and PA on genes involved in inflammation and ceramide metabolism. Since previous studies have shown that mild FA provokes long-term functional protection against PA-induced behavioral consequences [27], we also investigated if this long-lasting brain tolerance to asphyxia is mediated by changes in these genes. To our knowledge, the present study is the first to demonstrate that both FA and PA influence the prefrontal cortical ceramide pathway chronically.

Although FA and PA induce acute cytokines changes (Vlassaks et al., submitted), we could not detect any chronic changes in inflammatory genes. Genes involved in ceramide metabolism, however, revealed significant changes even 8 months after an asphyctic insult, suggesting that ceramide metabolism is very sensitive to alterations during early brain development. Although FA is considered milder than PA, with no fetal and neonatal mortality and normal cognitive behavior at adult age [27], both insults impact the chronic expression of Lass1 in the prefrontal cortex. Lass 1 is responsible for the conversion of sphinganine towards ceramide. Therefore, decreased Lass1 levels may lead to less ceramide synthesis resulting in less apoptosis $[147,148]$. In the FA-PA group, Lass1 mRNA levels were normal as compared with controls. This demonstrates that Lass1 levels are only changed after a single asphyctic insult, regardless its severity, maintaining normal apoptosis when two asphyctic insults are combined. Sphk1 mRNA was upregulated in the FA-PA group compared to the other conditions. SphK1 decreases ceramide and sphingosine levels and increases sphingosine-1-phosphate [149]. Accordingly, SphK1 upregulation causes more cell survival and proliferation $[49,150,151]$, which might be beneficial for memory processing [152].

The changes in ceramide metabolism genes in the prefrontal cortex, identified in this study, could have major complications later on since chronic changes in the ceramide pathway can accelerate aging and lead to neurodegenerative disorders [153]. Important to note is that 8 months of age may be too early to detect major changes in all brain regions. No behavioral changes were observed yet at 3 months of age (un- 
published results). At the age of 6 months, however, we detected lower locomotor activity and object recognition in PA animals [27]. Although several studies indicate anxiety- and emotion- related changes after PA [154,155], these changes were only observed at later time points than 8 months. Overall, these studies indicate that asphyxia induces long-term behavioral consequences but that these mechanisms need time to develop. Although the prefrontal cortex, hippocampus and cpu are all vulnerable to aging [156,157], these regions show selective vulnerability to several insults. Reason for this may relay in the density of N-methyl-D-aspartate (NMDA) and metabotropic glutamate (mGlu) receptors [158]. A higher NMDA binding site density is present in the hippocampus, while the cpu contains more mGluRs [158]. Consequently these regions may better cope with an asphyctic insult, while the prefrontal cortex may be more sensitive to asphyxia-induced excitotoxicity. Last, it is also important to note that we looked at animals that survived the asphyctic insults, so neuroprotective mechanisms may be activated in these animals as well.

In summary, FA and PA have region-specific, persisting effects on mRNA levels of ceramide genes. The data suggest that the combination of two subsequent asphyctic insults provides long-lasting neuroprotection in the prefrontal cortex probably by maintaining normal apoptosis and promoting cell proliferation. Deeper knowledge of the effects of asphyxia on genes involved in ceramide metabolism will help to understand the changes leading to brain tolerance and will open opportunities for the development of new therapies in the newborn. 



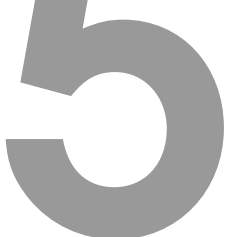

Inflammatory changes and ceramide profiles in rat liver after fetal asphyctic preconditioning and perinatal asphyxia

Evi VLASSAKS, Antonio W.D. GAVILANES, Maria NIKIFOROU, Eveline STRACKX, Otto BEKERS, Diego GAZZOLO, Giovanni LI VOLTI, Pilar MARTINEZ-MARTINEZ and Boris W. KRAMER

Submitted to PlosOne 


\section{ABSTRACT}

Objective To investigate acute and chronic hepatic inflammation, ceramide signaling and hepatocellular damage after both fetal (FA) and perinatal asphyxia (PA).

Study Design A clinical relevant rat model was used, inducing global asphyctic insults to reflect the human pathophysiology. At embryonic day 17, FA was induced by clamping the uterine vasculature for 30 minutes. PA was induced at day of birth by placing the uterine horns in a water bath for 19 minutes. Livers of newborn and adult rats were analyzed at several short- and long-term time points after both insults.

Results FA induced an acute inflammatory response that was associated with increased mRNA levels of ceramide transporters CERT and GPBP. At $96 \mathrm{~h}$ after FA, BAD mRNA levels and oxidative DNA damage decreased. Also after PA an inflammatory response was observed, with increased IL-6 and IL-10 mRNA levels $2 \mathrm{~h}$ after birth. The combination of FA and PA (preconditioning) attenuated the inflammatory response, reflecting comparable IL-6 and IL-10 mRNA levels as control animals. Moreover, $6 \mathrm{~h}$ after PA anti-apoptotic genes were downregulated associated with less lipid peroxidation, while preconditioned animals were comparable with controls. No clear differences in liver structure or hepatocellular damage could be observed after FA and/or PA for all investigated time points.

Conclusion Hepatic cytokine and ceramide levels are acutely changed after fetal and perinatal asphyxia. Asphyctic preconditioning seems to have an acute protective effect on the liver by modulating the inflammatory, apoptotic and anti-oxidative response. 


\section{INTRODUCTION}

Fetal (FA) and perinatal asphyxia (PA) are major causes of neurological disability and account for $23 \%$ of all neonatal deaths worldwide [142]. Due to impaired gas exchange between mother and fetus, fetal metabolic demands and cellular energy supplies are disturbed [159]. Consequently, severe asphyxia can result in hypoxicischemic encephalopathy (HIE) and multi-organ dysfunction like hepatic, heart and renal failure $[160,161]$.

Only few studies have investigated hepatic injury after asphyxia, although the liver is usually involved [162]. Human data revealed increased hepatic enzymes in serum of asphyxiated newborns $[162,163]$. Additionally, the enzyme concentrations positively correlated with the severity of HIE and higher mortality rates $[162,163]$. Furthermore, the concentrations of interleukin (IL)-6, IL-8 and IL-10 have been shown to be upregulated in the sera of asphyxiated neonates compared with those in healthy newborns [34]. In a rat model of global PA, Ashdown and coworkers reported increased IL-6 and IL- $1 \beta$ in the liver [35]. Overall, these studies indicate that PA leads to acute hepatocellular damage and systemic inflammation. These are important observations as the liver is a multifunctional organ with central roles in metabolism, biosynthesis, secretion and detoxification [164]. Moreover, early liver complications might lead to metabolic diseases in later life [165]. In this study, we aimed to investigate in more detail the acute and chronic hepatic inflammatory response after both FA and PA. In previous research, using a rat model of global asphyxia, we have already demonstrated cytokine changes after FA and PA in the brain (unpublished data). In adult rat models for hepatic ischemia/reperfusion (I/R) injury, cytokines have been demonstrated to be involved in necrotic and apoptotic death of hepatocytes $[164,166]$. Also ceramides have emerged as important lipid intermediates playing a role in the stress response and apoptotic cell death $[164,167]$. Moreover, knocking down ceramide generation attenuates hepatocellular damage [168] and hepatic inflammation [164]. These studies illustrate an important role of cytokines and ceramides in hepatic I/R damage, but no studies have been performed to elucidate their role in neonatal asphyctic liver injury. Moreover, to our knowledge, no studies addressed the consequences of asphyctic hepatic damage in adult life. 
Accordingly, we investigated hepatic inflammation, ceramide signaling and hepatocellular damage after FA and PA during neonatal and adult life in a well-established rat model for global asphyxia. Additionally, we assessed whether fetal asphyctic preconditioning (PC) would have any beneficial effect on the liver as it has already been shown to be neuroprotective $[27,28]$.

\section{MATERIALS AND METHODS}

\section{Animals and experimental procedures}

All experiments were approved and performed according to the Animal Ethics Board of Maastricht University on animal welfare according to Dutch governmental regulations. Timed-pregnant Sprague-Dawley rats (Charles River, France) were housed individually under standard laboratory conditions and food and water given ad libitum. Pregnant rats were randomly assigned to an experimental group. Unsexed fetuses, male neonates and male adults were used within this study.

FA was induced at embryonic day 17 (E17) by clamping both the uterine and ovarian arteries for 30 minutes with removable clamps as was previously explained [27]. At E21/22, PA was induced by submersing the uterine horns (containing the pups) in a saline bath $\left(37^{\circ} \mathrm{C}\right)$ for 19 minutes [27].

For the collection of tissue samples, pups and adult rats were sacrificed by decapitation at several time points after FA and at different short- and long-term time points after birth $(n=5 / 6)$ (Figure 1). Liver samples were collected from all offspring and plasma was taken from adult rats, snap-frozen in liquid nitrogen and preserved at $-80^{\circ} \mathrm{C}$ for further analysis.

\section{RNA extraction and RT-PCR}

Total RNA was prepared from liver tissue using Trizol Reagent (Invitrogen, Breda, the Netherlands), according to manufacturer's guidelines. Reverse transcription was carried out using $1 \mu \mathrm{g}$ of template RNA and the RevertAid First Strand cDNA Synthesis Kit (Fermentas, St. Leon Rot, Germany), according to manufacturer's instructions. 
$5 \mu \mathrm{l}$ of diluted cDNA (dilution 1:20) was amplified with LightCycler 480 SYBR Green I Master (Roche Applied Science, Almere, the Netherlands) in a final volume of $20 \mu \mathrm{l}$. Real-time PCR was performed using the Light Cycler 480 (Roche). Each PCR was carried out in duplicate and an RT-negative control was included for every sample. Values are reported relative to the geomean mRNA expression of three housekeeping genes (HPRT, $\beta$-actin and GAPHD). Primers were designed using Primer3plus (http://www.bioinformatics.nl/cgi-bin/primer3plus/primer3plus.cgi, Boston, USA) for interleukin (IL-) $1 \beta$, tumor necrosis factor (TNF)- $\alpha$, IL-10, IL-6, ceramide transport protein (CERT), goodpasture-antigen-binding protein (GPBP), LAG1 homolog ceramide synthase 1 (Lass1), neutral sphingomyelinase (nSMase), acid sphingomyelinase (aSMase), and sphingomyelin synthase 1 (SMS1); BAX, BAD, Bcl-2 and Bcl-XL. Quantification cycle values were extracted with the Lightcycler 480 software (Conversion LC and Linge PCR) and calculated based on the cycle threshold $(\mathrm{Ct})$ values.

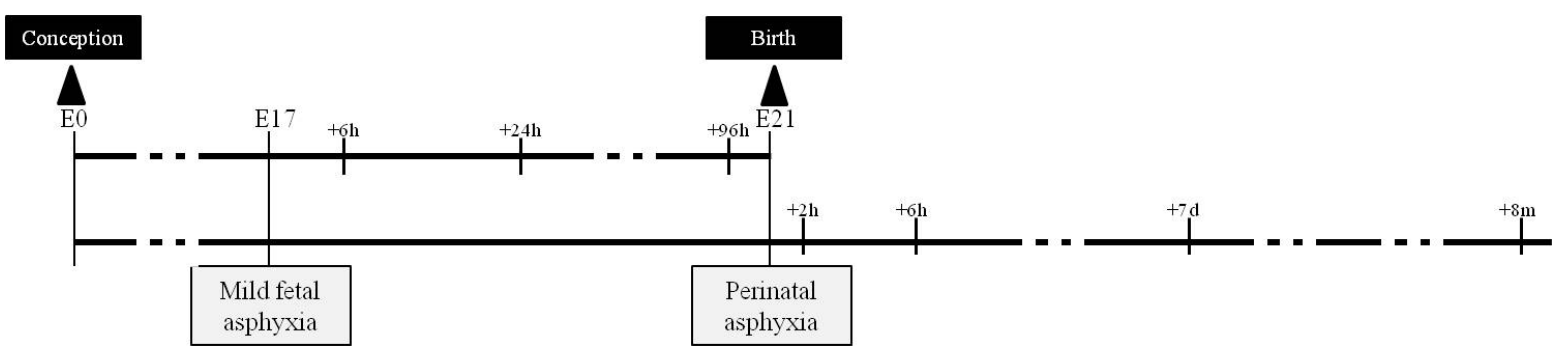

Figure 1: Experimental design. Fetal asphyxia was induced at E17 by clamping the uterine vasculature during 30 minutes. At term birth, global perinatal asphyxia was induced by placing the uterine horns containing the pups in a saline bath for 19 minutes. All animals were delivered by Caesarean section. Pups were euthanized at $6 \mathrm{~h}, 24 \mathrm{~h}$ and $96 \mathrm{~h}$ after FA ( $\mathrm{n}=5 / 6$ per group per time point) and $2 \mathrm{~h}, 6 \mathrm{~h}, 7$ days and 8 months after birth ( $\mathrm{n}=5 / 6$ per group per time point). $\mathrm{E}=$ embryonic day.

\section{Western Blot}

$10 \%$ SDS-PAGE gels were loaded with $40 \mu \mathrm{g}$ of total protein and transferred to a nitrocellulose membrane (Millipore, Amsterdam, the Netherlands). The membrane was blocked for $1 \mathrm{~h}$ with 5\% BSA in TBS-T and incubated with mouse mono- 
clonal anti-TNF $\alpha$ (diluted 1:500; sc-80383; Santa Cruz Biotechnology, Santa Cruz, USA) or rabbit polyclonal anti-CERT (diluted 1:2500; epitope 300-350 of human GPBP; Bethyl Laboratories, Montgomery, TX, USA) overnight at $4{ }^{\circ} \mathrm{C}$. After PBS washes, the membrane was incubated with donkey anti-mouse (diluted 1:10 000; Rockland Immunochemicals, Gilbertsville, USA) and goat anti-rabbit (diluted 1:10 000, Rockland Immunochemicals, Gilbertsville, USA) for $1 \mathrm{~h}$ at room temperature. Finally, the membrane was washed with PBS, dried and scanned using the LICOR Odyyssey infrared imaging system.

\section{Immunohistochemistry}

Snap-frozen livers were cut $\left(7 \mu \mathrm{m}\right.$ at $\left.-13^{\circ} \mathrm{C}\right)$ and mounted on gelatin coated slices (VWR International). General Hematoxylin and Eosin staining was performed to visualize general liver structure. Whole sections were analyzed for inflammation and scored in a blinded manner by a specialized animal pathologist. The sections from each animal were scored as zero if they had no inflammatory cells present in tissue, one for a few inflammatory cells (1-20 cells), two for moderate cell infiltration (21-40 cells), three for a large number of inflammatory cells (41-60 cells) and four if inflammation was spread all over in the tissue ( $>61$ cells) [80]. To investigate fat deposition in the adult liver, frozen $7 \mu \mathrm{m}$-thick sections were stained with oil red-O [169].

\section{Plasma parameters}

In order to assess hepatic function at the age of 8 months, aspartate aminotransferase (AST), alanine aminotransferase (ALT), alkaline phosphatase (ALP) and total bilirubine were determined on a Beckman Coulter Synchron LX20 PRO Clinical Chemistry analyzer (Beckman Coulter, Fullerton, USA). The activity of AST, ALT and ALP were measured by an enzymatic rate method whereas bilirubine was measured using the timed-endpoint Diazo method. 


\section{Total Thiol Groups (GSH) and 8-OH-dG determination}

Tissue levels of total thiol groups (GSH) were measured, in $200 \mu \mathrm{l}$ of tissue homogenate, using a spectrophotometric assay based on the reaction of thiol groups with 2,2-dithio-bis-nitrobenzoic acid (DTNB) at $\lambda=412 \mathrm{~nm}\left(\varepsilon \mathrm{M}=13600 \mathrm{M}^{-1} \mathrm{x}\right.$ $\mathrm{cm}^{-1}$, where $\varepsilon \mathrm{M}$ is a wavelength-dependent molar absorbtivity coefficient). The limit of detection for this assay is approximately $15 \mathrm{nM}$. The intra-assay coefficient of variation is $4 \%$ whereas the inter-assay coefficient of variation is $5.6 \%$.

8-OHdG, a surrogate marker of DNA damage, was measured by a commercially available kit (Cayman Chemicals, Ann Harbor, MI) according to the manufacturer's instructions.

\section{Statistical analysis}

Statistical analysis was performed with GraphPad Prism software (version 4.0; GraphPad Software Inc.) and Statistical Package for the Social Sciences (SPSS version 17.0 for Windows). Data are represented as mean + standard error of the means (SEM). For each parameter, normality was tested using a KolmogorovSmirnov test. Prenatal data were analyzed using Student's t-test. All postnatal data were analyzed using one-way analysis of variance (ANOVA) test, followed by posthoc comparisons using LSD correction. Statistical significance was established at $\mathrm{p}<0.05$ and indicated by *.

\section{RESULTS}

\section{PRENATAL EFFECTS AFTER FA}

To have a longitudinal overview of the hepatic inflammatory response after FA, we analyzed prenatal livers at 6 h, $24 \mathrm{~h}$ and $96 \mathrm{~h}$ after $30 \mathrm{~min}$ of global FA (Figure 1). The body and liver weights of FA animals were not different from controls at all evaluated time points (data not shown). 


\section{FA induces an acute inflammatory response and changes in ceramide genes}

An acute inflammatory response was observed, with increased IL-1 $\beta$ mRNA levels $6 \mathrm{~h}$ after FA ( $\mathrm{p}=0.042$; Figure 2A) and elevated IL-6 mRNA levels $24 \mathrm{~h}$ after FA compared with control levels ( $\mathrm{p}=0.05$; Figure $2 \mathrm{~B}$ ). No significant changes between control and FA animals were observed in TNF- $\alpha$ and IL-10 mRNA levels (data not shown).

The increased IL-6 mRNA levels at $24 \mathrm{~h}$ after FA were associated with increased mRNA levels of both ceramide transporters CERT ( $\mathrm{p}=0.027$; Figure 2C) and GPBP $(p=0.014$; Figure 2D), indicating more ceramide synthesis. This was supported by Lass1 mRNA levels that tended to increase $24 \mathrm{~h}$ after FA ( $\mathrm{p}=0.094$; Figure 2E). Later in time at $96 \mathrm{~h}$ after FA, a trend towards increased aSMase $(\mathrm{p}=0.064$; Figure $2 \mathrm{~F})$ and higher SMS1 levels $(\mathrm{p}=0.003$; Figure $2 \mathrm{G})$ were observed, pointing to changes in sphingomyelin metabolism.

\section{Decreased oxidative DNA damage $96 \mathrm{~h}$ after FA}

To investigate whether the changes in inflammatory response and ceramide metabolism were associated with hepatocellular damage, we assessed mRNA levels of the apoptotic genes BAX, BAD, Bcl-2 and Bcl-XL. In addition, we looked at glutathione $(\mathrm{GSH})$ as measure for lipid peroxidation $[170,171]$ and 8-hydroxy-2-deoxy Guanosine $(8-\mathrm{OH}-\mathrm{dG})$ as marker of oxidative DNA damage $[172,173]$ as these have been shown to be important in the development of post-ischemic liver damage [174]. Most important changes were observed at $96 \mathrm{~h}$ after FA with significant decreased BAD levels after FA compared with control levels $(p=0.034$; Figure 3B). This decrease in BAD mRNA levels was associated with decreased levels of 8-OH-dG $(\mathrm{p}=0.036$; Figure 3C), indicating less oxidative DNA damage $96 \mathrm{~h}$ after FA. Surprisingly, no changes in GSH oxidation could be observed for all investigated time points (Figure 3D). $96 \mathrm{~h}$ after the FA insult, we also assessed general liver morphology by $\mathrm{H} / \mathrm{E}$-staining. No differences in inflammatory cell infiltration or general liver structure could be observed comparing livers of control and FA pups (data not shown). 
A

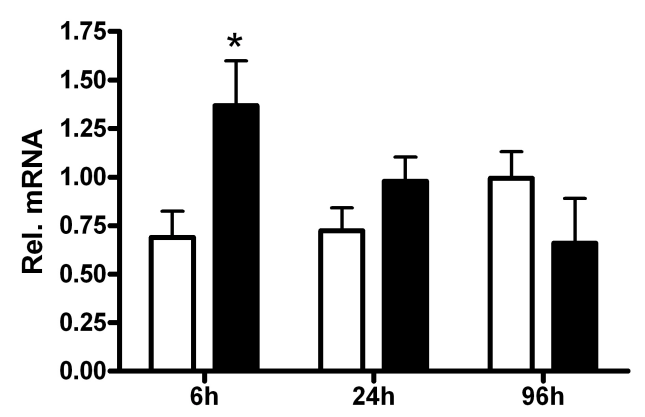

C

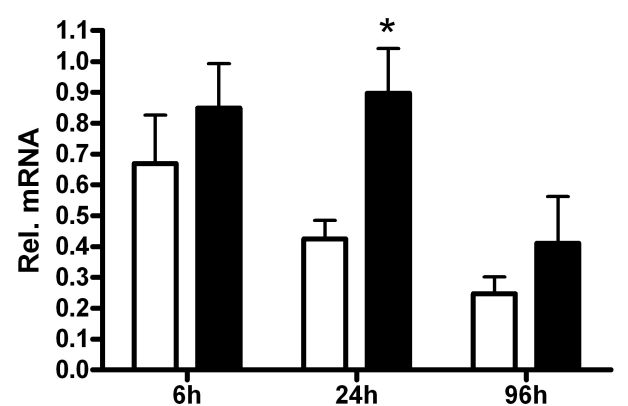

$\mathbf{E}$

Lass1

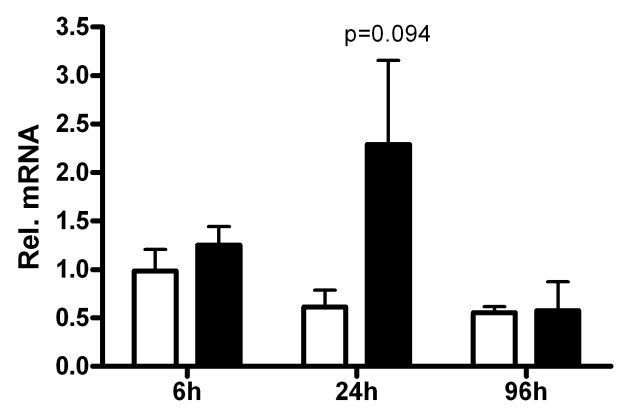

G

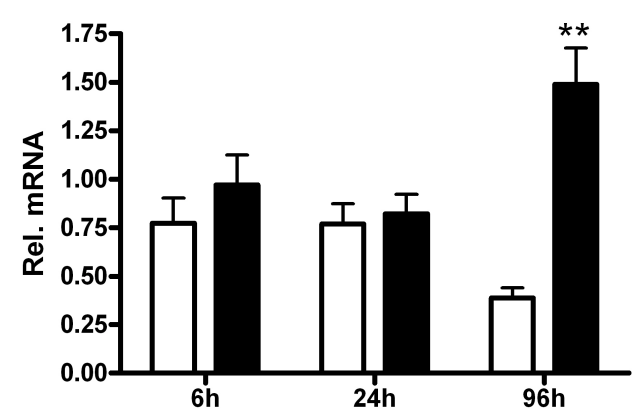

B

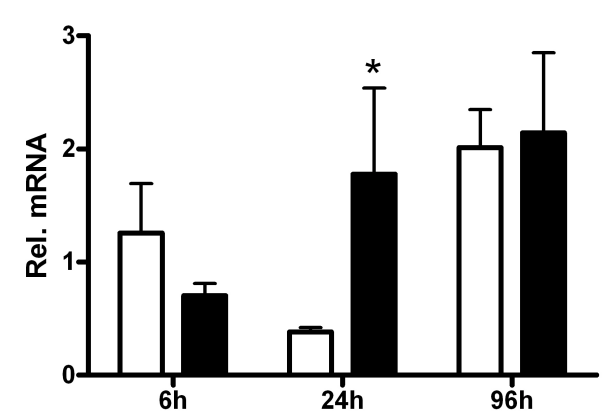

D GPBP

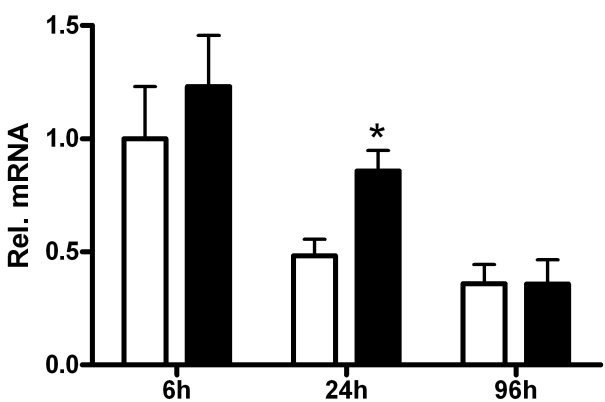

$\mathbf{F}$

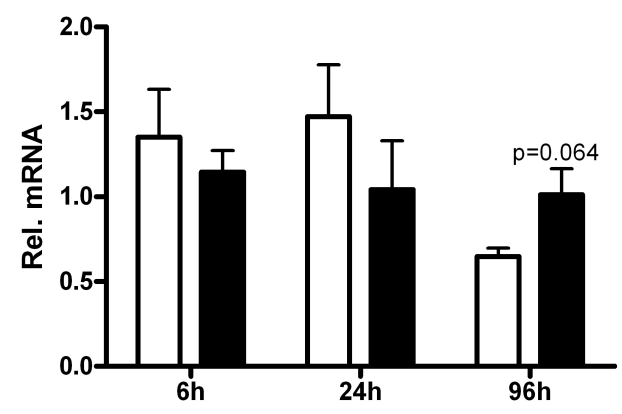

Figure 2: FA induced prenatal changes in mRNA levels of inflammatory cytokines and ceramide genes. Prenatal mRNA levels of IL-1 $\beta$ (A), IL-6 (B), CERT (C), GPBP (D), Lass1 (E), aSMase (F) and SMS1 (G) in control and FA animals. mRNA levels are relative to the geomean of $\beta$-actin, HPRT and GAPDH. $* \mathrm{p}<0.05$ and $* * \mathrm{p}<0.01$ significantly different from respective control group. Data shown as mean + SEM. 

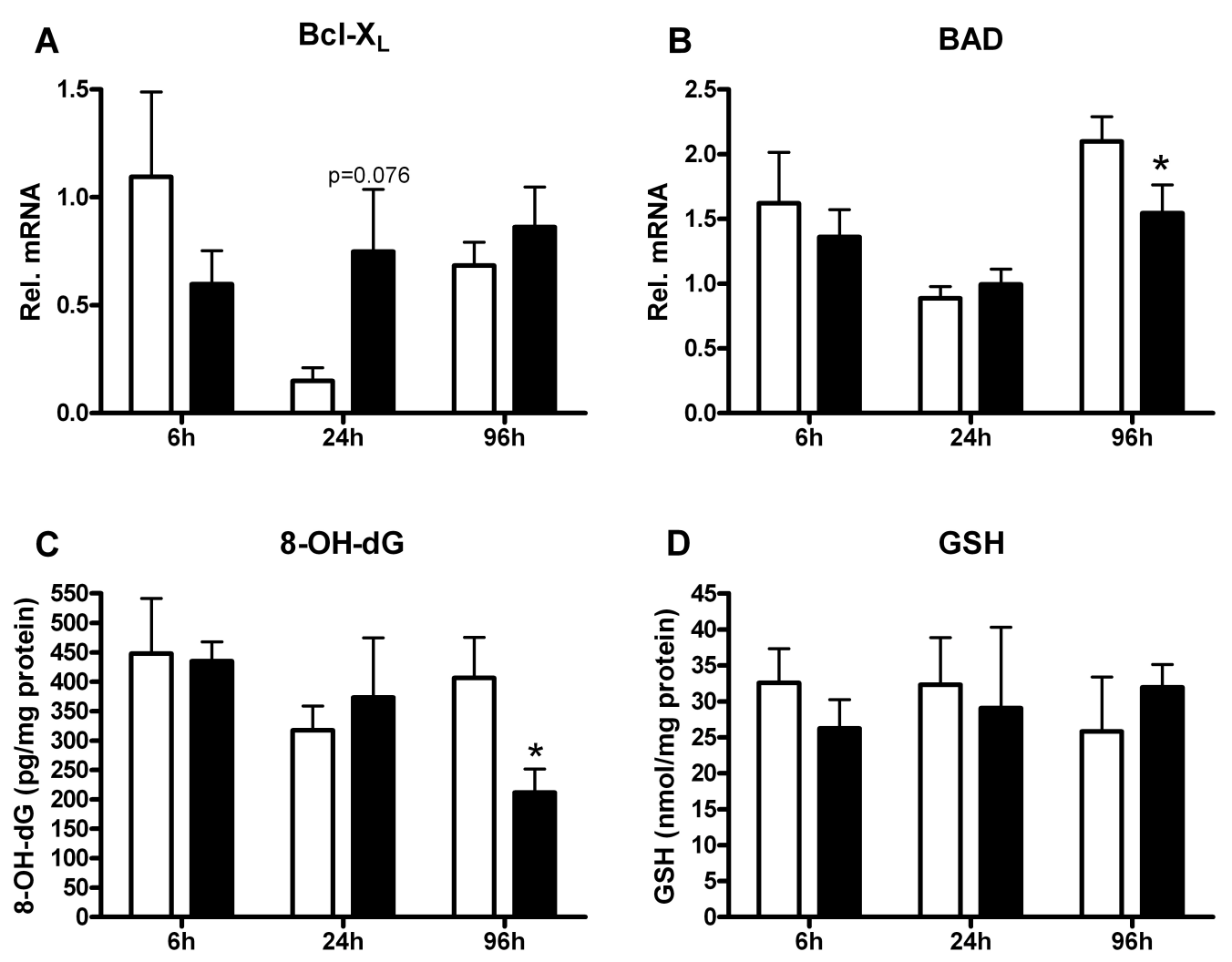

Figure 3: Hepatic apoptosis and oxidative DNA damage seem to decrease $96 \mathrm{~h}$ after FA. Prenatal mRNA levels of Bcl-XL (A) and BAD (B) in control and FA animals. mRNA levels are relative to the geomean of $\beta$-actin, HPRT and GAPDH. Oxidative DNA damage measured by $8-\mathrm{OH}-\mathrm{dG}$ levels (C) and total thiol groups (GSH) (D) corrected for their protein content. ${ }^{*} \mathrm{p}<0.05$ significantly different from respective control group. Data shown as mean + SEM.

\section{ACUTE POSTNATAL EFFECTS AFTER PA AND PC}

At $2 \mathrm{~h}, 6 \mathrm{~h}$ and 7 days after birth, we assessed hepatic inflammation, ceramide signaling and hepatocellular damage after PA and PC (Figure 1). At $6 \mathrm{~h}$ after birth, pups that experienced PA (with or without the combination of FA) weighted significantly less than control pups (Table 1). However, at postnatal day 7 this effect was normalized again. Liver weights did not differ among groups at all measured time points (Table 1). 


\section{Increased IL-6 and IL-10 levels $2 \mathrm{~h}$ after PA and changes in ceramide metabolism $6 \mathrm{~h}$ after PA}

$2 \mathrm{~h}$ after birth, low levels of pro-inflammatory cytokines IL- $1 \beta$ and TNF- $\alpha$ were observed compared with the other time points. When animals were exposed to both FA and PA (PC group) levels of these cytokines were even lower ( $\mathrm{p}=0.059$ and $\mathrm{p}=0.05$ respectively; Figure $4 \mathrm{~A}+\mathrm{B})$. IL- 6 mRNA levels were significantly increased $2 \mathrm{~h}$ after PA relative to controls ( $\mathrm{p}<0.001$; Figure 4C). This increased IL-6 expression was attenuated when animals were previously exposed to FA (PC group; $\mathrm{p}<0.001$ for PA vs. PC; Figure 4C). Comparable results were found for IL-10, with increased mRNA levels $2 \mathrm{~h}$ after PA but baseline levels in PC animals ( $\mathrm{p}=0.036$ for C vs. PA and $\mathrm{p}=0.048$ for PA vs. PC; Figure 4D).

$6 \mathrm{~h}$ after birth, changes in cytokine mRNA levels were observed in the PC animals with a trend to decreased IL- $1 \beta$ levels ( $\mathrm{p}=0.076$; Figure $4 \mathrm{~A})$ and increased IL-10 levels ( $\mathrm{p}<0.001$; figure 4D) compared with control and PA animals. Also aSMase levels tended to decrease in PC animals compared with control levels ( $p=0.053$; Figure 4E, right panel). For SMS1, decreased mRNA levels were seen $6 \mathrm{~h}$ after PA ( $p=0.055$; Figure $4 F$, right panel), while PC animals showed baseline levels.

\section{Decreased lipid peroxidation $6 \mathrm{~h}$ after PA}

$6 \mathrm{~h}$ after PA, BAD and Bcl- 2 mRNA levels decreased ( $\mathrm{p}=0.056$ and $\mathrm{p}=0.05$ respectively; Figure 5A), while PC animals showed baseline levels. Bcl-XL mRNA levels were higher in the PC group compared with both control $(\mathrm{p}=0.015)$ and PA animals $(\mathrm{p}=0.004$; Figure 5A). These changes in apoptotic mRNA levels were associated with increased levels of GSH ( $\mathrm{p}=0.01)$ and $8-\mathrm{OH}-\mathrm{dG}$ (not significant) in PA animals. PC animals showed comparable levels as controls (Figure 5B+C). Based on $\mathrm{H} / \mathrm{E}$-staining we could not observe changes in hepatocellular damage between all groups at the $6 \mathrm{~h}$ time point (data not shown). 

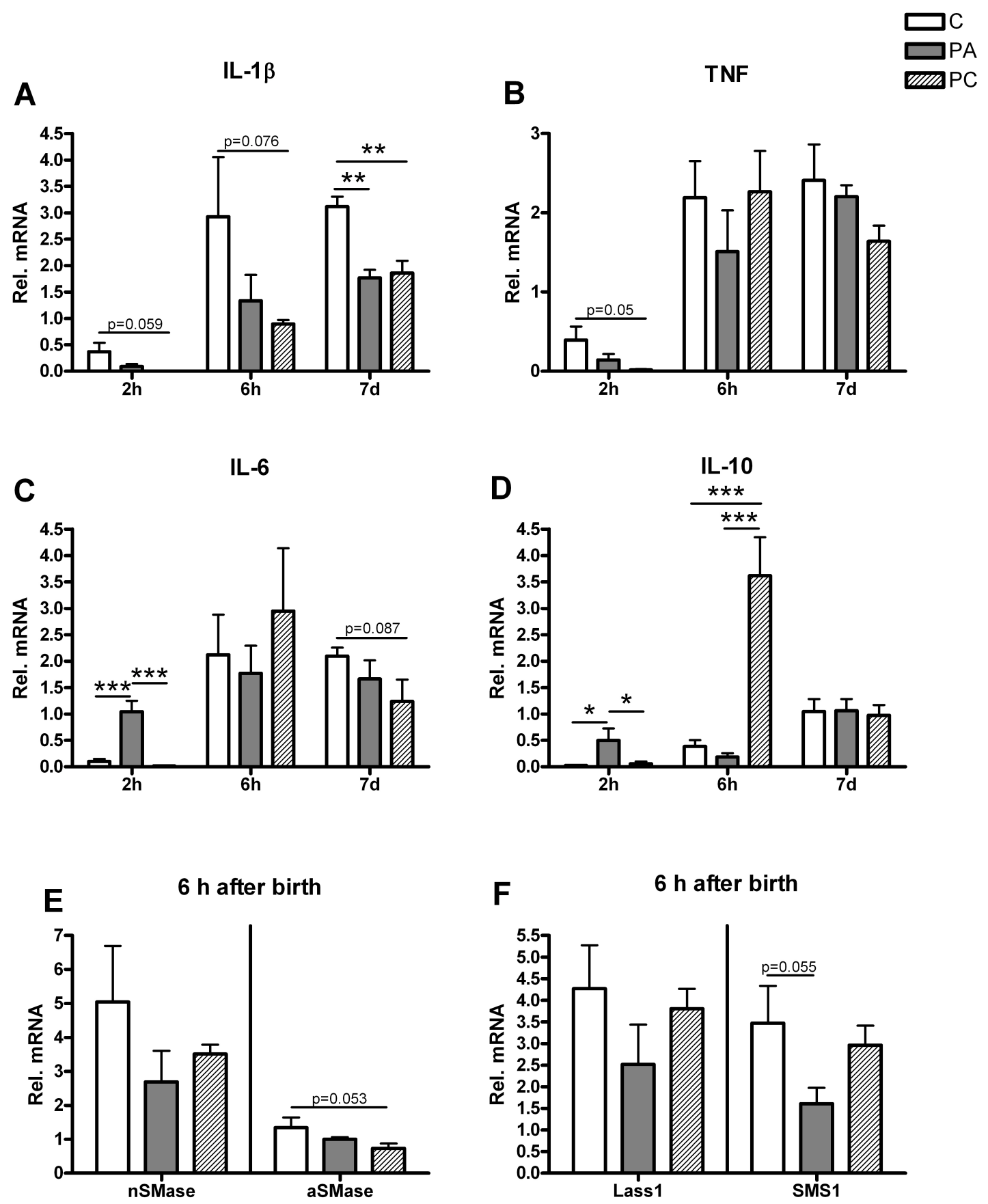

Figure 4: Increased IL-6 and IL-10 mRNA levels in PA animals, but baseline IL-6 and IL-10 mRNA levels in preconditioned animals and changes in ceramide metabolism genes $6 \mathrm{~h}$ after birth. Acute postnatal mRNA levels of IL-1 $\beta$ (A), TNF- $\alpha$ (B), IL-6 (C), IL-10 (D), nSMase and aSMase (E), Lass1 and SMS1 (F), in control, PA and PC animals. mRNA levels are relative to the geomean of $\beta$-actin, HPRT and GAPDH. ${ }^{*} \mathrm{p}<0.05$; $* *$ $\mathrm{p}<0.01$ and $* * * \mathrm{p}<0.001$ indicate significant differences between groups. Data shown as mean + SEM. 

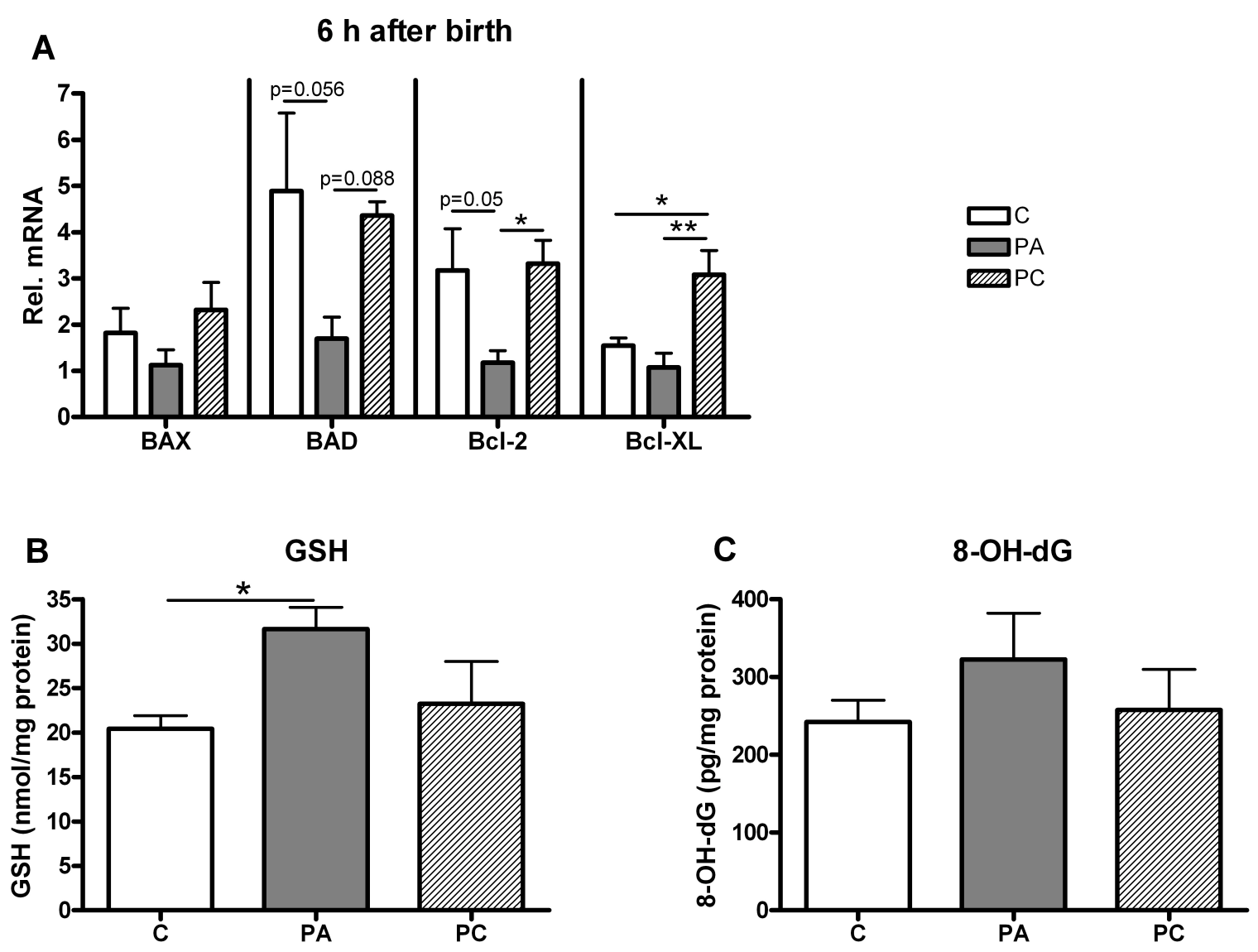

Figure 5: Decreased expression of anti-apoptotic genes and lipid peroxidation $6 \mathrm{~h}$ after PA. Postnatal mRNA levels of BAX, BAD, Bcl-2 and Bcl-XL are presented relative to the geomean of $\beta$-actin, HPRT and GAPDH (A). Lipid peroxidation (GSH) (B) and oxidative DNA damage measured by 8-OH-dG levels $(\mathrm{C})$ corrected for their protein content at $6 \mathrm{~h}$ after birth. $* \mathrm{p}<0.05$ and $* * \mathrm{p}<0.01$ indicate significant differences between groups. Data shown as mean + SEM.

\section{CHRONIC EFFECTS}

To assess whether FA and PA also have hepatic consequences in adulthood, we analyzed livers of 8 month old animals. PA animals weighted significantly less than control and PC animals (Table 1). Liver weights, however, did not differ between groups (Table 1). 
Table 5.1: Liver and body weights after birth of control, PA and PC animals. Weights are presented as average \pm SEM. * Significantly different from respective control group.

\begin{tabular}{|c|c|c|c|c|c|c|}
\hline & \multicolumn{3}{|c|}{ 2 hours } & \multicolumn{3}{c|}{ 6 hours } \\
\cline { 2 - 8 } & Control & PA & PC & Control & PA & PC \\
\hline Liver weight (g) & $0.12 \pm 0.01$ & $0.07 \pm 0.01$ & $0.17 \pm 0.03$ & $0.09 \pm 0.01$ & $0.08 \pm 0.01$ & $0.13 \pm 0.01$ \\
\hline Body weight (g) & $5.69 \pm 1.02$ & $4.37 \pm 1.01$ & $5.62 \pm 0.12$ & $7.10 \pm 0.20$ & $5.50 \pm 0.34 *$ & $5.52 \pm 0.08 *$ \\
\hline Ratio Liver/Body weight (\%) & 2.11 & 1.60 & 3.02 & 1.27 & 1.45 & 2.36 \\
\hline \multirow{2}{*}{} & \multicolumn{7}{|c|}{$\mathbf{7}$ days } & & & $\mathbf{8}$ months & \\
\cline { 2 - 8 } & Control & PA & PC & Control & PA & PC \\
\hline Liver weight (g) & $0.21 \pm 0.02$ & $0.29 \pm 0.03$ & $0.25 \pm 0.01$ & $19.77 \pm 1.08$ & $17.86 \pm 0.30$ & $19.20 \pm 1.29$ \\
\hline Body weight (g) & $15.60 \pm 4.86$ & $18.61 \pm 2.73$ & $14.36 \pm 2.52$ & $604.33 \pm 18.63$ & $560.7 \pm 10.17 *$ & $602.63 \pm 14.64$ \\
\hline Ratio Liver/Body weight $(\%)$ & 1.35 & 1.56 & 1.74 & 3.27 & 3.19 & 3.19 \\
\hline
\end{tabular}

\section{No changes in inflammatory response and ceramide metabolism at 8 months of age}

No significant differences between groups were observed in hepatic mRNA levels for all cytokines and ceramide enzymes (data not shown). In the PC group, the expression of CERT was significantly decreased compared with PA animals $(\mathrm{p}=0.049)$ and showed a declining trend compared with controls $(\mathrm{p}=0.07$; Figure 6A). GPBP was higher expressed in both PA $(\mathrm{p}=0.036)$ and $\mathrm{PC}$ animals $(\mathrm{p}=0.082)$ compared with controls (Figure 6B). Western Blot analysis of CERT and GPBP did not reveal any significant changes (data not shown).

\section{Increased plasma levels of ALP in PC animals at 8 months of age}

At 8 months, measurements of genes of the BAX family and lipid peroxidation revealed no significant changes between groups (data not shown). Yet, 8-OH-dG levels seemed to be higher in animals that experienced PA during birth (both PA and PC animals) although levels did not reach significance (Figure 6C). Signifi- 
cance was reached for plasma levels of ALP, showing higher levels in PC animals $(\mathrm{p}=0.03$ ) compared with controls (Figure 6D). Nevertheless, no significant changes were observed in plasma levels of AST and ALT (Figure 6E $+6 \mathrm{~F}$ ).

The general liver structure based on H/E-staining was not different between groups. Overall, we observed a normal liver structure with no enlargement of the hepatocytes or compression of the sinusoids, and no hepatic inflammatory cell infiltration in all experimental groups. Also no changes in intracellular lipid accumulation were observed, based on Oil Red O staining (data not shown).

\section{COMMENT}

This is the first study demonstrating that FA and PA induce an acute hepatic inflammatory response. The inflammatory response after FA is mainly associated with changes in ceramide metabolism, while after birth ceramide metabolism did not seem to be affected. Interestingly, acutely after birth it seems that FA preconditioning may induce hepatic protection by modulating the inflammatory, apoptotic and anti-oxidative response.

Perinatal asphyxia can cause hepatic hypoxic injury in newborn infants [162,175]. However, the exact mechanisms underlying this hepatic damage are not defined. Studies suggest that hepatic changes only occur in most severe cases of asphyxia $[12,162,176]$. Conversely, animal models show that even short periods of hypoxia can induce leakage of cytosolic liver enzymes [177]. More insights into the underlying mechanisms and consequences of hypoxic hepatic damage are needed since the liver has important roles in metabolism, biosynthesis, secretion and detoxification [164]. Moreover, disturbed hepatic function might lead to metabolic diseases in later life [165].

PA is associated with a systemic inflammatory response as is indicated by increased serum concentrations of IL-6, IL-8 and IL-10 in asphyxiated neonates [34] and increased IL-6 and IL-1 $\beta$ in the asphyctic rat liver [35]. Furthermore, ceramides are important in hepatocellular damage [167] and are upregulated by inflammatory mediators like cytokines $[45,174]$. Hence, in this study we aimed to 

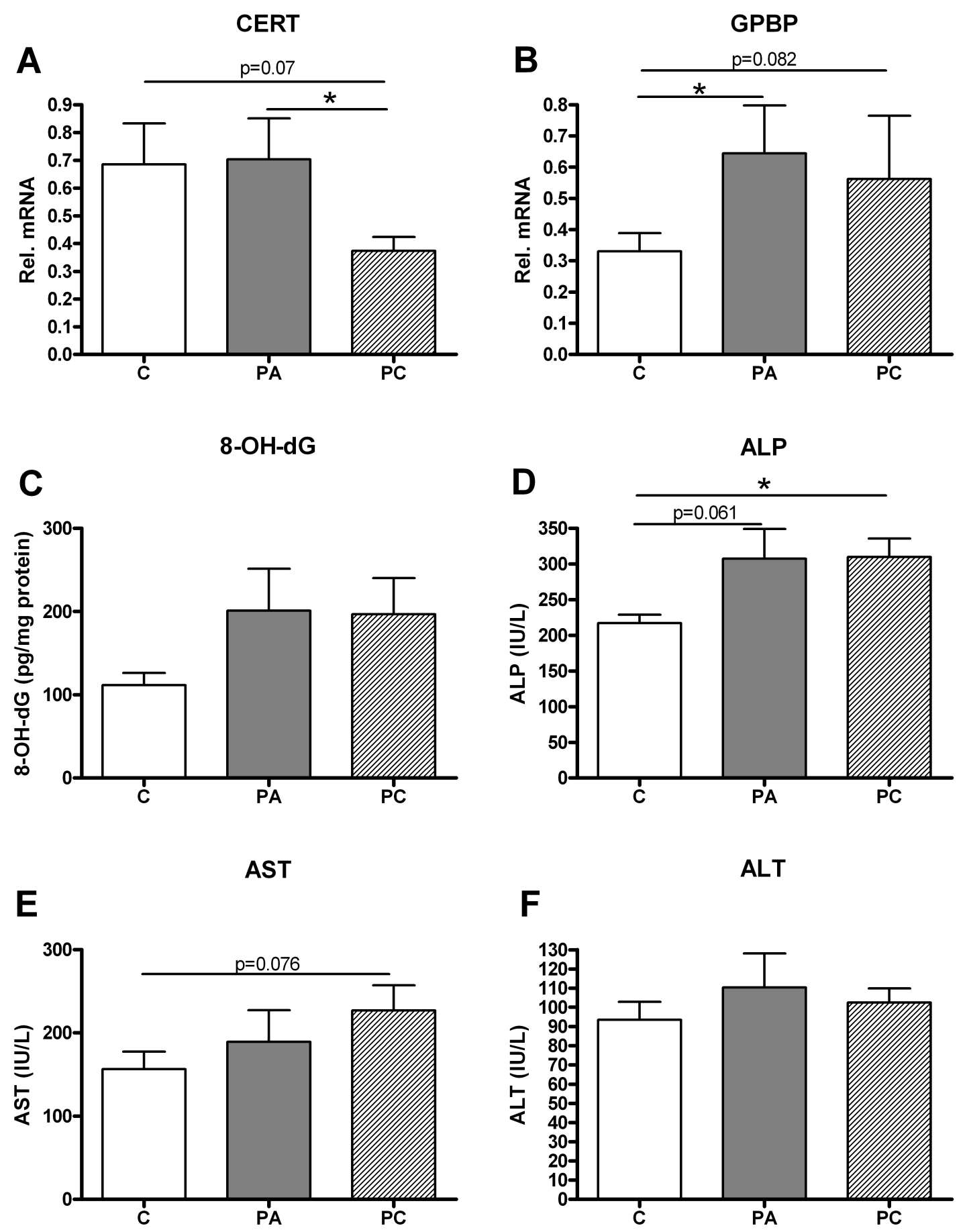

Figure 6: ALP levels are increased in PC animals at 8 months of age. mRNA levels of CERT (A) and GPBP (B) at 8 months of age in control, PA and PC animals. Levels are presented relative to the geomean of $\beta$-actin, HPRT and GAPDH. 8-OH-dG levels corrected for their protein content (C), plasma ALP (D), AST (E) and ALT (F) levels in U/L of control, PA and PC animals at 8 months of age. $* \mathrm{p}<0.05$ and $* * \mathrm{p}<0.01$ indicate significant differences between groups. Data shown as mean + SEM. 
investigate in more detail the hepatic inflammatory response after both fetal and perinatal asphyxia. We examined if this inflammatory response was associated with changes in ceramide metabolism and hepatocellular damage. We also considered if FA and PA have any long-term hepatic impact. Finally, we assessed whether asphyctic preconditioning would have any beneficial effect on the liver.

An acute inflammatory response was observed after FA, initiated by increased mRNA levels of IL-1 $\beta$ and followed by increased expression of IL-6. In previous research, we showed that FA downregulates the acute inflammatory response in the brain (unpublished results). Later on, at $96 \mathrm{~h}$ after the asphyctic insult, brain IL-6 mRNA was also increased. The different expression in brain and liver in response to the same asphyctic insult shows that different inflammatory responses are generated in various tissues. The acute and increased inflammatory response in the liver is not surprising since the liver possesses the largest population of resident macrophages in the body and the liver is known to produce inflammatory cytokines in response to ischemia and hypoxia [35]. Similar results were reported by Ashdown and coworkers, who found in a perinatal setting increased IL- 6 and IL- $1 \beta$ in the rat liver $2 \mathrm{~h}$ after global PA [35]. Although TNF- $\alpha$ is a major mediator in the adult hepatic response to I/R [174], we could not find any significant changes in this cytokine in our model for perinatal global asphyxia. However, we did find changes in IL- $1 \beta$, a cytokine with the same physiological effects as TNF- $\alpha$ [39]. IL- $1 \beta$ induces IL-6 production [32,42] and is associated with ceramide metabolism [45]. At $24 \mathrm{~h}$ after FA we found increased levels of Lass1 at the same time point as the increased IL-6 levels, assuming increased ceramide levels. In addition, the mRNA levels of the transporters CERT and GPBP were upregulated. These transporters are responsible for transporting ceramide from the endoplasmatic reticulum (ER) to the Golgi. In the Golgi, ceramide can be metabolized to sphingomyelin by the enzyme SMS [48]. In our study, SMS1 was upregulated $96 \mathrm{~h}$ after FA. Also aSMase, an enzyme that is responsible for sphingomyelin breakdown, was higher expressed at this time point. aSMase-induced ceramide generation is attributed to be important in I/R liver injury [168]. It has been shown that when aSMase activity is increased after I/R this leads to hepatic lipid peroxidation and DNA degradation [174]. The 
higher expression of aSMase in our study was not associated with lipid peroxidation and DNA damage. Instead, we found lower levels of apoptotic mRNA expression and downregulated levels of 8-OH-dG. Important to mention is that we induced a global sublethal asphyctic insult, while studies reporting I/R hepatic damage perform specific ischemia on the liver inducing more severe damage in this organ. Also worthwhile to mention is that when we observe the decreased levels in BAD and $8-\mathrm{OH}-\mathrm{dG}$, the fetuses are primed to be born (E21 time point). Therefore, we assume that this downregulation in apoptosis and DNA damage might be a hepatic protective mechanism in the fetus.

This protective effect was indeed seen at two hours after birth in preconditioned animals concerning the inflammatory response. Hepatic inflammation was induced $2 \mathrm{~h}$ after PA compared with controls with increased mRNA levels of IL-6 and IL-10. As was also observed in the brain (unpublished results), the increased inflammatory response was absent in the preconditioned FA-PA animals, showing comparable mRNA levels as control animals. The cytokines IL-6 and IL-10 are known to be secreted together $[33,42]$ and are higher expressed in cerebrospinal fluid (CSF) and serum from newborn infants with asphyctic encephalopathy $[32,34]$. The precise role in asphyctic injury is not clear. Both cytokines are considered to have anti-inflammatory properties [113-116]. On the other hand, higher IL-6 levels in response to asphyxia are correlated with higher mortality rates [33]. These results indicate an important role of IL- 6 and IL-10 in the inflammatory response after birth asphyxia. More research is needed to elucidate the specific role of these cytokines in global asphyxia.

Interestingly, also at six hours after birth a possible protective effect of FA preconditioning on the liver was observed. Preconditioned animals showed baseline levels of Bcl-2, while expression of this anti-apoptotic gene was downregulated in PA animals. In a study of Maulik et al., it has been shown that Bcl-2 regulates the inhibition of apoptosis after ischemic preconditioning [178]. Also Bcl-XL has been attributed to play an important role in preconditioning induced protection since overexpression of Bcl-XL prevented apoptosis in cortical and hippocampal neurons in a neonatal and adult mouse model of hypoxia/ischemia $[179,180]$. This observation might by applicable in our model as well since preconditioned ani- 
mals showed high upregulation of this gene. Additionally, at $6 \mathrm{~h}$ after birth, PA animals showed lower expression of SMS1, the enzyme that converts ceramide to sphingomyelin [48]. Although this SMS1 downregulation was not associated with increased levels of aSMase, the data suggest that less ceramide is converted to sphingomyelin. Consequently, ceramide levels can accumulate and induce lipid peroxidation [174]. Although I/R injury in the liver is known to coincide with increased lipid peroxidation [174], we could not validate this in our perinatal model of global asphyxia since increased GSH levels were observed. We assume that the upregulation of anti-oxidant products is an endogeneous protective mechanism of the neonatal livers to counterbalance the asphyctic damage.

At 8 months of age the liver still seemed to be affected by FA and PA. Nevertheless, only minor changes were observed with no significant consequences for normal liver pathology.

In summary, we showed that PA induces hepatic inflammation with higher cytokine levels of IL-6 and IL-10. Asphyctic preconditioning may modulate the inflammatory, apoptotic and anti-oxidative response thereby protecting the neonate from PA damage. More studies are needed to investigate the long-term consequences of global perinatal asphyxia on the liver. 



\section{6}

\section{Chorioamnionitis induced hepatic inflammation and disturbed lipid metabolism in fetal sheep}

Veerle BIEGHS*, Evi VLASSAKS*, Anne CUSTERS*, Patrick J. VAN GORP, Marion J. GIJBELS, Aalt BAST, Otto BEKERS, Luc J. I. ZIMMERMANN, Dieter LÜTJOHANN, J.-Willem VONCKEN, A.W. Danilo GAVILANES, Boris W. KRAMER and Ronit SHIRI-SVERDLOV

* These authors contributed equally to this paper.

Pediatr Res. 2010 Dec; 68(6):466-472 


\section{ABSTRACT}

Chorioamnionitis frequently induces a fetal inflammatory response syndrome (FIRS), characterized by an elevation of pro-inflammatory mediators and systemic inflammation. Although there is increasing evidence that inflammation and lipid metabolism influence each other, the effects of chorioamnionitis-induced FIRS on fetal lipid homeostasis are currently not known. Accordingly, we hypothesize that chorioamnionitis induces an inflammatory response in the fetal liver, consequently leading to metabolic disturbances. Chorioamnionitis was induced by intra-amniotic injection of $10 \mathrm{mg}$ endotoxin two days or two weeks before delivery. Saline injections were given to controls. The effect of chorioamnionitis on hepatic inflammation and metabolic parameters was analyzed in ovine fetuses at the gestational age (GA) of 125 days (normal $\mathrm{GA}=150$ days). We found that two days after the endotoxin injections, inflammatory markers were significantly higher compared to controls. Additionally, lipid and glucose metabolism were disturbed in response to endotoxin. Moreover, the anti-oxidant state capacity was reduced and hepatic damage was apparent. Two weeks after the endotoxin injections the fetal livers were still inflamed and had higher glucose concentrations in the blood. In addition, the levels of markers for hepatic damage (ALT, AST) were elevated. In conclusion, chorioamnionitis induces liver inflammation, leading to metabolic disturbances in the fetus. 


\section{INTRODUCTION}

Preterm delivery is a major problem in reproductive medicine today. Currently, approximately 10 percent of all deliveries are preterm [66]. Irrespective of the progress in neonatal care, preterm delivery is still the most important cause of neonatal death and long-term neurological morbidity. In addition, most interventions to prevent preterm birth are unsuccessful $[59,181]$.

Chorioamnionitis, defined as inflammation of the amniotic fluid and fetal membranes caused by bacterial endotoxin, is a common pregnancy complication and is associated with preterm birth [60]. At least 60 percent of all preterm births below 25 weeks of gestation have mothers that suffer from chorioamnionitis [59, 65]. Chorioamnionitis frequently causes a fetal inflammatory response syndrome (FIRS), which is characterized by an elevation of pro-inflammatory mediators and systemic inflammation [61]. So far, most studies in the field of chorioamnionitis and FIRS have focused on the effects on lungs and brain [61]. However, the effects of chorioamnionitis-induced FIRS on the liver have thus far not been investigated. The liver is a central organ in an organism's metabolism and damage to the liver may play an important role in the development of metabolic diseases $[88,89]$. Accordingly, we hypothesized that chorioamnionitis induced an inflammatory response in the fetal liver, which would lead to fetal metabolic disturbances. This hypothesis is in line with several observations indicating that inflammation and lipid metabolism influence each other [182]. Besides the finding that lipids can induce an inflammatory state, inflammation itself has been described to induce changes in lipid metabolism [182].

To test our hypothesis, we examined whether intra-amniotic endotoxin injection in sheep, an established model of chorioamnionitis [67, 74, 80-82, 95, 96] induced inflammation and lipid changes in the fetal liver. Both short term (two days) and long term (two weeks) effects of endotoxin-induced chorioamnionitis were studied. The livers of ovine fetuses were analyzed at birth at a GA of 125 days (comparable with a GA of 28 weeks in humans; normal ovine $\mathrm{GA}=150$ days) for haematopoietic activity, anti-oxidative capacity, glycogen storage, inflammation and cell damage. In addition, we studied the relationship between hepatic inflammation and lipid levels in the liver and glucose concentrations in plasma. 


\section{MATERIALS AND METHODS}

\section{Animals, intra-amniotic injections and tissue processing at delivery}

The present study protocol was approved by and was performed according to the guidelines of the Animal Care Committee of the University of Maastricht, The Netherlands. Time-mated Texel ewes, bearing both singletons and twins, were randomly assigned to receive a single dose of $10 \mathrm{mg}$ endotoxin (Escherichia coli 055:B5; Sigma Chemical, St. Louis, MO) or the equivalent volume of saline for controls by ultrasound guided intra-amniotic injections [80]. The influence of singleton/twin on the analyzed variable was ruled out by using multivariate analysis of variance.

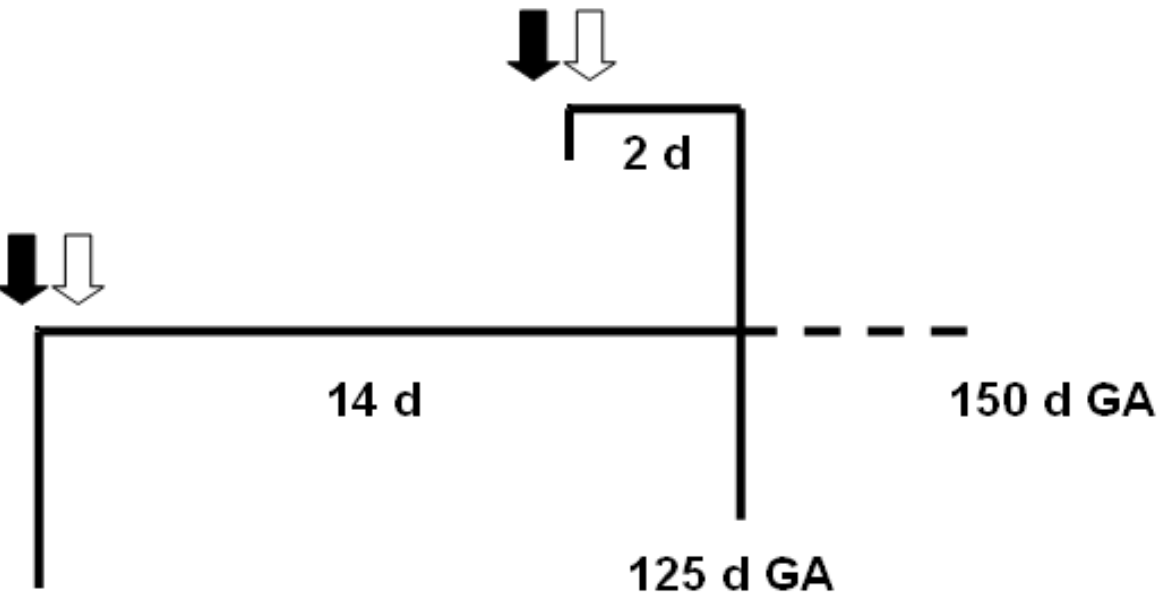

\section{Intra-amniotic endotoxin \\ \Intra-amniotic saline (control)}

Figure 1: Schematic overview of the experimental design. Chorioamnionitis was induced by intra-amniotic injections of endotoxin two days $(\mathrm{GA}=123$ days, $\mathrm{n}=5)$ and two weeks $(\mathrm{GA}=111$ days, $\mathrm{n}=7)$ before delivery at GA 125 days (normal $\mathrm{GA}=150$ days). Saline injections were given two days $(n=5)$ or 2 weeks before delivery $(n=7)$. Endotoxin injections are indicated as black arrows, saline injections are indicated as white arrows. 
Both short and long term effects of chorioamnionitis induced by intra-amniotic endotoxin injections were studied two days $(n=5)$ and two weeks $(n=7)$ before the Caesarean section delivery at the GA of 125 days (term being 150 days; Figure 1). At birth, a cord blood arterial sample was collected followed by a lethal injection of pentobarbital sodium via the umbilical vein. Samples of the left liver were either fixated in $4 \%$ formaldehyde or snap-frozen in liquid nitrogen for further analysis.

\section{Immunohistochemistry and histological analysis}

Liver tissue was fixed in formaldehyde, embedded in paraffin and cut in $3 \mu \mathrm{m}$ sections. Hepatic inflammation and haematopoiesis were evaluated after haematoxylin and eosin (H/E) staining in response to endotoxin-induced chorioamnionitis. Whole sections were analyzed for inflammation and scored in a blinded manner by a specialized animal pathologist. The sections from each animal were scored as zero if they had no inflammatory cells present in tissue, one for a few inflammatory cells (1-20 cells), two for moderate cell infiltration (21-40 cells), three for a large number of inflammatory cells (41-60 cells) and four if inflammation was spread all over in the tissue ( $>61$ cells) [80]. The sections were also scored for haematopoiesis, using the following semiquantitative scale: grade 0 (none), grade 1 (mild), grade 2 (moderate) and grade 3 (severe) [183]. The specificity of the inflammatory cells in the fetal liver tissue was determined by immunohistochemical staining for T-lymphocytes (CD3) and for myeloperoxidase (MPO) [184]. Endogenous peroxidase activity was blocked by incubation in hydrogen peroxide. Nonspecific binding sites were blocked with serum. After antigen retrieval, CD3-positive T-lymphocytes were stained with a polyclonal rabbit anti-human CD3 antibody (DAKO A0452, dilution 1:200). Polyclonal rabbit antihuman MPO (DAKO A0398, dilution 1:500) was used as primary antibody to identify MPO-positive cells. Biotinylated polyclonal swine anti-rabbit IgG (DAKO E0353, dilution 1:200/1:500) was applied as secondary antibody. After adding StrepABComplex/HRP (DAKO KO377), staining was performed with 3-amino-9ethylcarbazole and sections were counterstained with haematoxylin. Sections were photographed at 200x magnification using a Nikon digital camera DMX1200 and ACT-1 v2.63 software from Nikon Corporation. For the CD3 and MPO immuno- 
histochemical staining, cell numbers were counted in 6 randomly selected microscopical views and were noted as cells $/ \mathrm{mm}^{2}$. Glycogen content was detected in the fetal livers by a Periodic Acid Schiff (PAS)-staining. Paraffin-sections were incubated in $1 \%$ periodic acid. Counterstaining was performed with haematoxylin. The relative glycogen content of individual livers was estimated from the PAS staining intensity, that was rated as negative (score 0), minimal (score 1), moderate (score 2) or severe (score 3). To investigate fat deposition in the liver, frozen $7 \mu \mathrm{m}$ thick sections were stained with oil red-O. Briefly, liver cryosections were fixed for 60 minutes in $3.7 \%$ formaldehyde solution and stained with $0.2 \%$ oil red-O in $60 \%$ triethyl-phosphate for 30 minutes. Sections were counterstained with Mayer's haematoxylin, washed with running tab water and covered with coverslip using $10 \%$ glycerol in PBS.

\section{RNA extraction and RT-PCR}

Total RNA was isolated from frozen liver samples by using Tri-reagent of Sigma, as previously described [89]. All applications were done according to manufacturer's protocols. Reverse transcription was performed using the iScript cDNA synthesis kit (Bio-Rad, Hercules, USA) according to manufacturer's instructions. Real-time PCR was performed on a Bio-Rad MyIQ with the IQ5 v2 software using the IQ SYBR Green Supermix with fluorescein (Bio-Rad, Hercules, USA) and $10 \mathrm{ng}$ of cDNA. Primers for target genes interleukin (IL)-1 $\beta$, IL-8, tumor necrosis factor (TNF) and glutathione S-transferase (GST), were developed using Primer Express version 2.0 (Applied Biosystems, Foster City, CA, USA) using default settings. To standardize for the amount of cDNA, cyclophylin A (peptidylprolyl isomerase A, ppia) was used as housekeeping gene. Primer sequences are given in table 1 . The liver real-time PCR results were obtained using the relative standard curve method.

\section{Anti-oxidant capacity}

The trolox equivalent anti-oxidant capacity (TEAC) indicates the total anti-oxidant status. The assay was performed as previously described with minor modifications [185]. In short, a 2,2'-azino-bis-3-ethylbenzthiazoline-6-sulphonic acid (ABTS)solution was prepared freshly. This solution was heated at $70^{\circ} \mathrm{C}$ for approximately 
$10 \mathrm{~min}$. The absorbance at $734 \mathrm{~nm}$ was determined every 2-3 min to evaluate the ABTS-formation. At an absorbance between 0.6 and 0.7 the reaction was stopped by cooling the solution on ice.

\section{Liver lipid analysis}

Approximately $50 \mathrm{mg}$ of frozen liver tissue was homogenized [89]. Protein content was measured using the bicinchoninic acid method (Pierce, Rockford, USA). Triglycerides and total cholesterol content were measured according to manufacturer's instructions on a Benchmark 550 Micro-plate reader (Bio-Rad, Hercules, USA).

\section{Plasma parameters}

Triglycerides, total cholesterol and HDL-cholesterol have been determined on a Beckman Coulter Synchron LX20 PRO Clinical Chemistry analyzer (Beckman Coulter, Fullerton, USA). The measurements have been validated in adult and fetal sheep. LDL-cholesterol has been calculated by the Friedewald equation [186, 187]. This equation estimates LDL-cholesterol from the measurements of total cholesterol, triglycerides and HDL-cholesterol; LDL-cholesterol = total cholesterol (triglycerides/2.2 + HDL-cholesterol) [188]. The glucose concentration was determined by an oxygen rate method employing the oxidation of glucose to gluconolactone catalyzed by glucose oxidase at an enzyme electrode.

GGT, AST and ALT have been determined on a Beckman Coulter Synchron LX20 PRO Clinical Chemistry analyzer (Beckman Coulter, Fullerton, USA), where the activity was measured by an enzymatic rate method. The rate of change in absorbance, which is caused by the enzymatic reaction, is directly proportional to the activity of GGT, ALT and AST in the sample and is used by the Synchron system to calculate and express the respective activity.

\section{Data analysis}

Data were statistically analyzed using Statistical Package for Social Sciences (SPSS 15.0 Software). Comparisons between controls and endotoxin-injected groups were performed by student t-test and in addition multivariate one-way analysis of vari- 
ance (ANOVA) with gender and singleton/twin as covariates. Significant effects were analyzed by post-hoc Bonferroni corrections. Scoring of hematopoietic, inflammatory cells and glycogen was calculated by Pearson's $\chi^{2}$-test. Results are presented as means $+/-$ SD and a p-value $<0.05$ was considered as statistically significant. $* / 11$ indicate significant differences between saline and endotoxin-injected groups with $*=\mathrm{p}<0.05 ; * *=\mathrm{p}<0.01 ; \|=\mathrm{p}<0.001$.

\section{RESULTS}

\section{Hepatic inflammation and haematopoiesis}

$\mathrm{H} / \mathrm{E}$ stained liver sections from control animals and animals that received endotoxin two days before delivery showed no differences in inflammatory cell infiltration (Figure 2). However, an increased level of infiltrated inflammatory cells was detected two weeks after endotoxin exposure (Figure 2A). There were no significant differences in haematopoiesis between the four groups (Table 2).

Consistent with the results of the H/E staining, CD3-positive T-lymphocytes were significantly increased in the livers of animals that received endotoxin two weeks before delivery (Figure 2B). Remarkably, most CD3-positive T-lymphocytes were positioned in the hematopoietic clusters (Figure 2 D-G). An interesting observation was the increase in the number MPO-positive cells both two days and two weeks after the endotoxin injections compared to controls (Figure 2C). Unlike the CD3-positive T-lymphocytes, the MPO-positive cells were not located in between the hematopoietic clusters (Figure $2 \mathrm{H}-\mathrm{K}$ ).

\section{Expression profiling of genes involved in inflammation}

To investigate the hepatic response to endotoxin induced chorioamnionitis in more detail, mRNA levels of TNF, IL- $1 \beta$ and IL- 8 were determined. TNF and IL- $1 \beta$ mRNA levels increased rapidly in the fetal liver of animals that were sacrificed two days after the endotoxin injection. Two weeks after the endotoxin exposure, cytokine mRNA levels went back to basal levels (Figure 3). mRNA levels for the neutrophil chemoattractant IL-8 did not change in the fetal liver after fetal exposure to endotoxin (Table 2). 
A

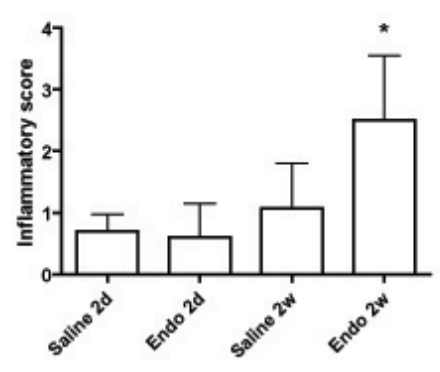

$E$

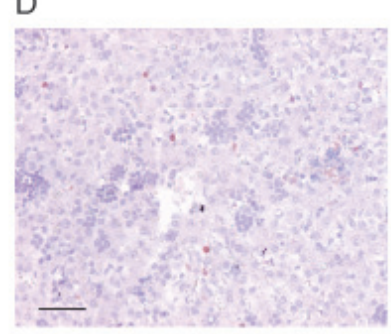

$\mathrm{H}$

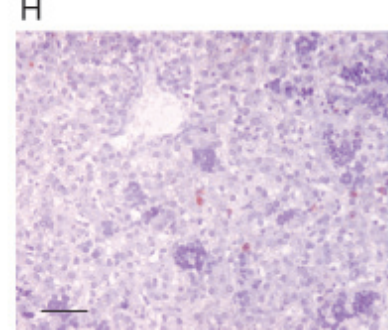

B

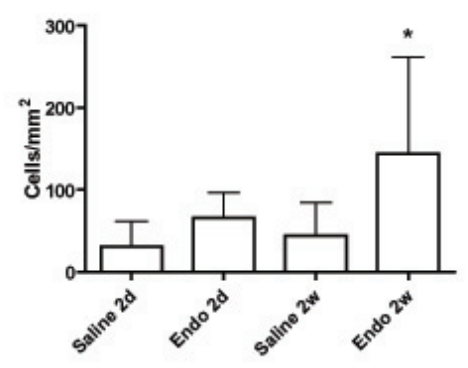

$\mathrm{F}$

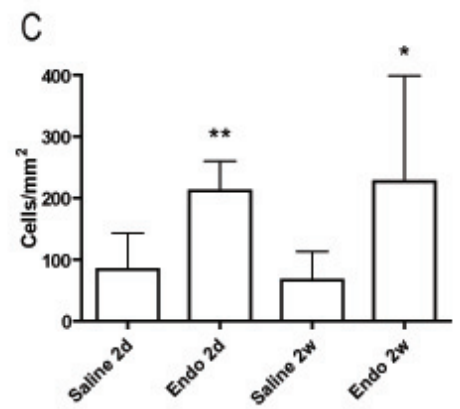

G

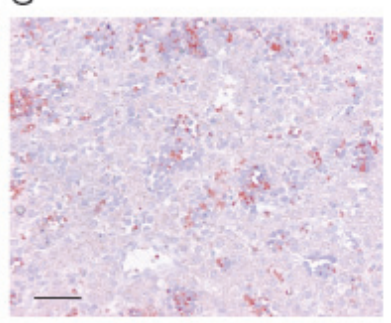

$\mathrm{K}$

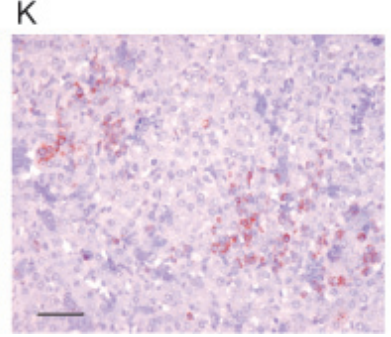

$\mathrm{J}$

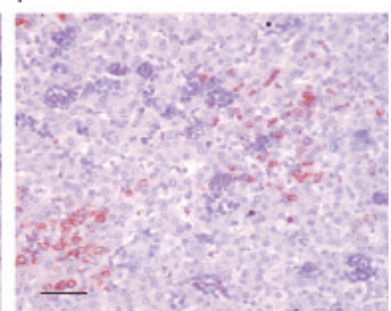

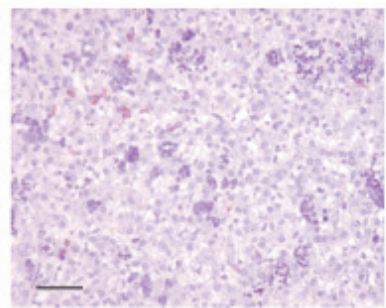

Figure 2: Hepatic inflammation. Inflammatory score of $\mathrm{H} / \mathrm{E}$ stained liver sections from saline and endotoxin (endo) exposed animals (A). T-lymphocyte staining (B) and MPO staining (C). Liver paraffin sections were stained against T-lymphocytes (CD3) and MPO respectively. Results are expressed as number of cells per $\mathrm{mm}^{2}$. Representative pictures (200x magnification; scale bar $100 \mu \mathrm{m}$ ) of the CD3 staining for the saline two days (2d) (D), endotoxin two days (E), saline two weeks $(2 \mathrm{w})(\mathrm{F})$ and endotoxin two weeks exposed animals respectively $(\mathrm{G})$. Representative pictures (200x magnification; scale bar $100 \mu \mathrm{m}$ ) of the MPO staining for the saline two days $(\mathrm{H})$, endotoxin two days (I), saline two weeks (J) and endotoxin two weeks $(\mathrm{K})$ exposed animals respectively. $*=\mathrm{p}<0.05$; $* *$ $=\mathrm{p}<0.01$ Significant different from saline injected group. 

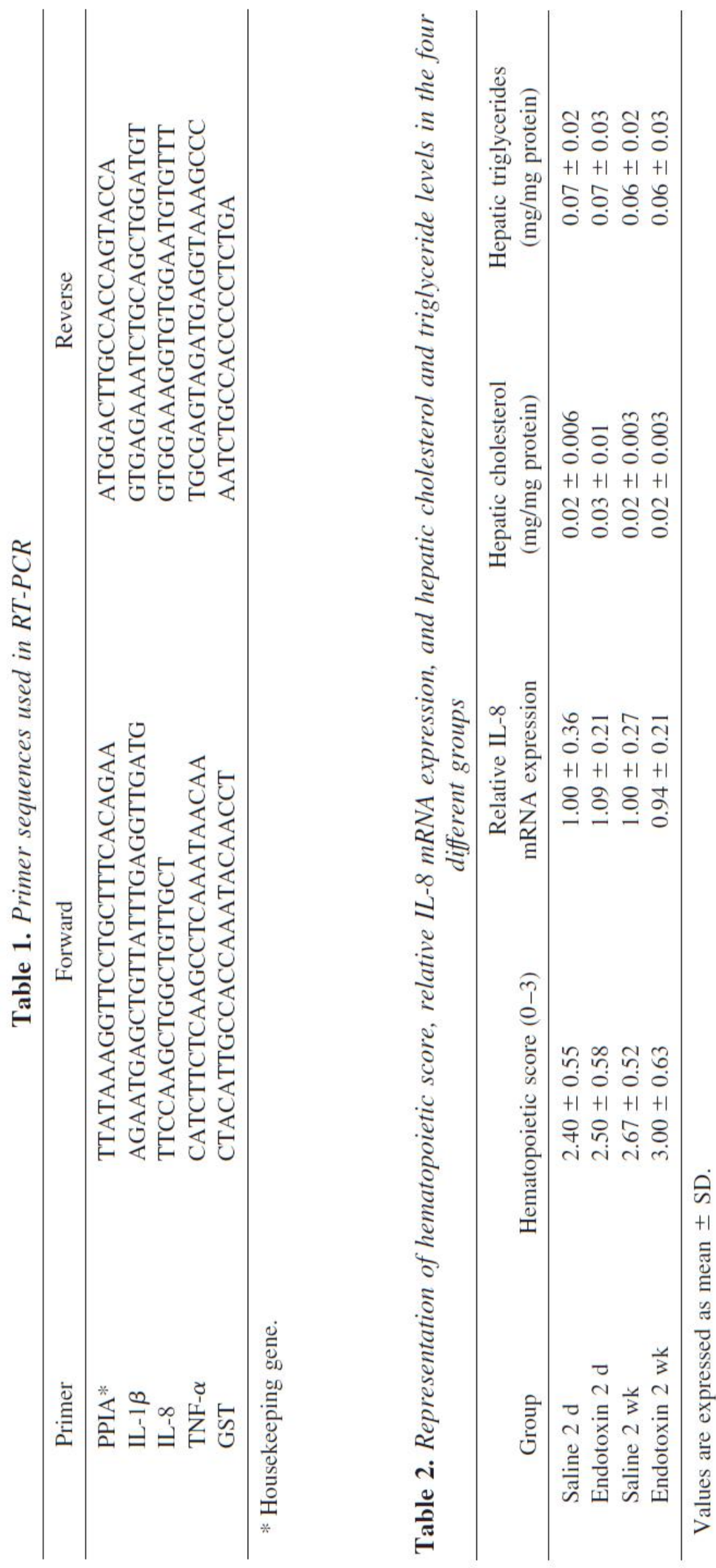
A

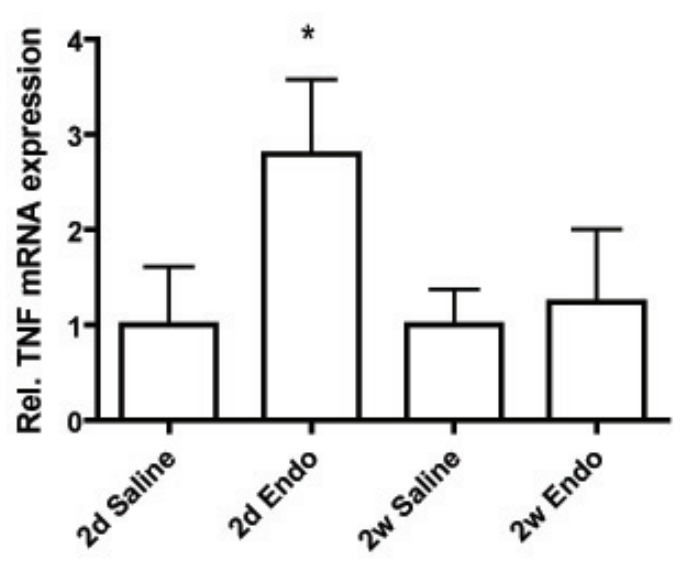

B

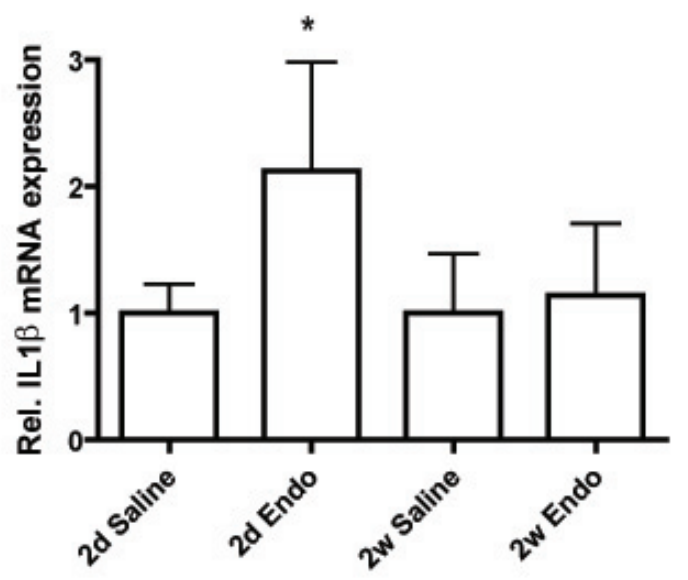

Figure 3: Hepatic gene expression analysis with quantitative reverse-transcription PCR. Cytokine mRNA levels were normalized to cyclophylin A (ppia). Relative gene expression of TNF (A) and IL-1 $\beta$ (B) in liver two days ( $2 \mathrm{~d}$ ) and two weeks $(2 \mathrm{w})$ after endotoxin (endo) compared to proper saline control. $*=p<0.05$ Significant different from saline injected group.

\section{Metabolic changes after endotoxin-induced chorioamnionitis}

Since the liver has indispensable functions in lipid homeostasis [189] and a correlation exists between lipid levels and inflammation [88, 190], we assessed the potential effect of hepatic inflammation on both hepatic and plasma lipid levels. Both total cholesterol and triglyceride plasma levels were increased two days after endotoxin-induced chorioamnionitis (Figure 4A+B). The increase in cholesterol corresponds with increased HDL and LDL levels, although only HDL levels are significantly increased two days after endotoxin-induced chorioamnionitis (Figure 4A). The increases in cholesterol and triglycerides were limited to the plasma. Two days after endotoxin exposure, preterm delivered lambs showed hepatic cholesterol levels that tended to increase compared to control levels $(\mathrm{p}=0.06)$. Two weeks after endotoxin injections, hepatic cholesterol went back to basal levels (Table 2). Hepatic triglycerides levels were not affected by exposure to endotoxin; this was independently confirmed by oil red-O staining (data not shown). 
A significant increase was observed in plasma glucose levels both two days and two weeks after the endotoxin injections (Figure 4D). In addition, abundant glycogen storage was observed within the livers of both control groups. However, the PAS staining in hepatocytes gradually disappeared after endotoxin injections (Figure $4 \mathrm{C}$ ), indicating that the amount of hepatic glycogen decreases in response to endotoxin-induced chorioamnionitis (Figure 4E-H).

\section{Liver damage after endotoxin-induced chorioamnionitis}

All groups exhibited equal anti-oxidant activities based on the TEAC assay, except for the group that was injected two days before delivery where we detected a decrease in the concentration of the anti-oxidant capacity (Figure 5A). Similarly, the measurement of mRNA expression of GST was also decreased two days after fetal exposure to endotoxin. Two weeks after the endotoxin injection, the mRNA expression returned to basal levels (Figure 5B).

To assess whether the hepatic inflammatory response and lipid changes were associated with increased liver damage, the level of plasma gamma-glutamyltranferase (GGT), Alanine aminotransferase (ALT) and aspartate aminotransferase (AST) were measured. Plasma GGT levels were significantly increased both two days and two weeks after endotoxin injections (Figure 5C). Both plasma ALT and AST measurements showed a significant increase two weeks after endotoxin-induced chorioamnionitis (Figure 5D+E). 

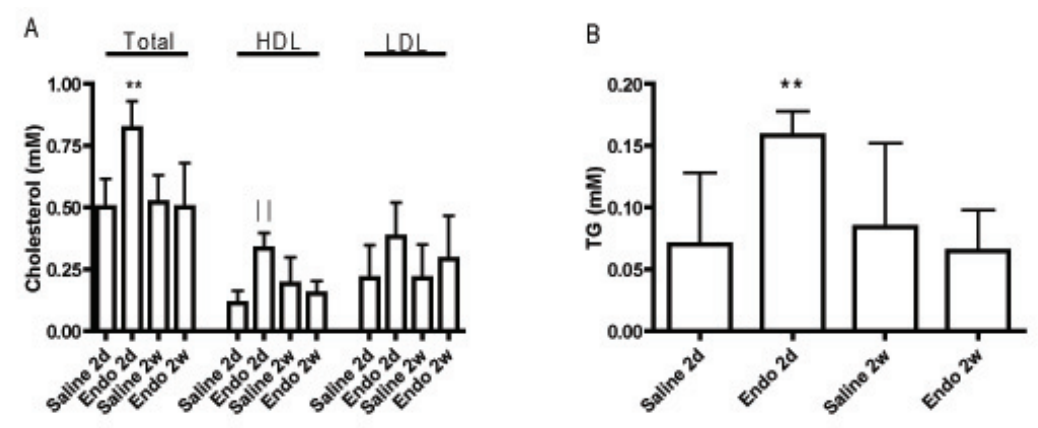

C

D
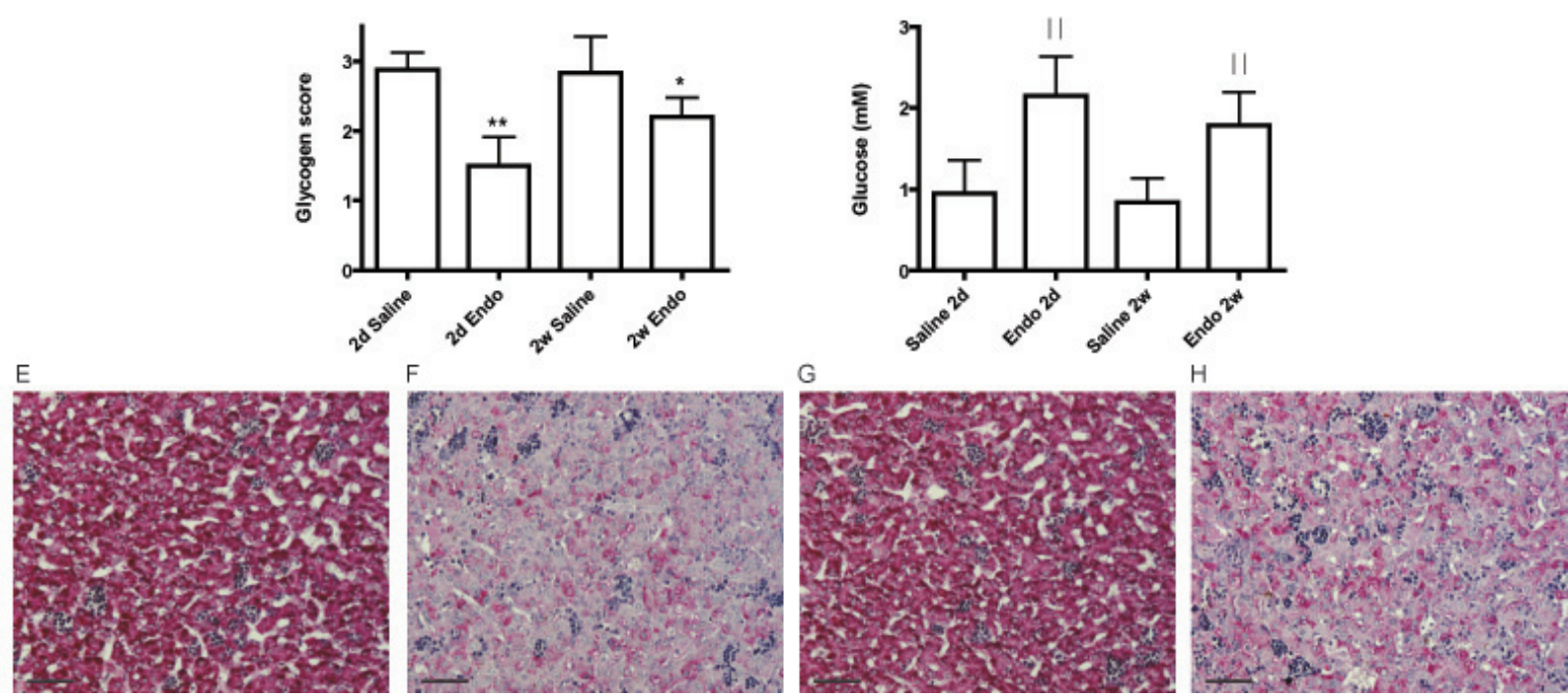

Figure 4: Metabolic parameters of saline and endotoxin (endo) exposed animals. Plasma total cholesterol, HDL and LDL levels (A). Plasma triglycerides levels (B). Representative score of hepatic glycogen content from control and endotoxin (endo) exposed animals based on PAS-stained liver sections (C). Plasma glucose levels (D). Representative pictures (200x magnification; scale bar corresponds to $100 \mu \mathrm{m}$ ) of the PAS staining for the saline two days $(\mathrm{E})$, endotoxin two days $(\mathrm{F})$, saline two weeks $(\mathrm{G})$ and endotoxin two weeks $(\mathrm{H})$ exposed animals respectively. $*=\mathrm{p}<0.05$; ** $=\mathrm{p}<0.01 ; \|=\mathrm{p}<0.001$ Significant different from saline injected group. 

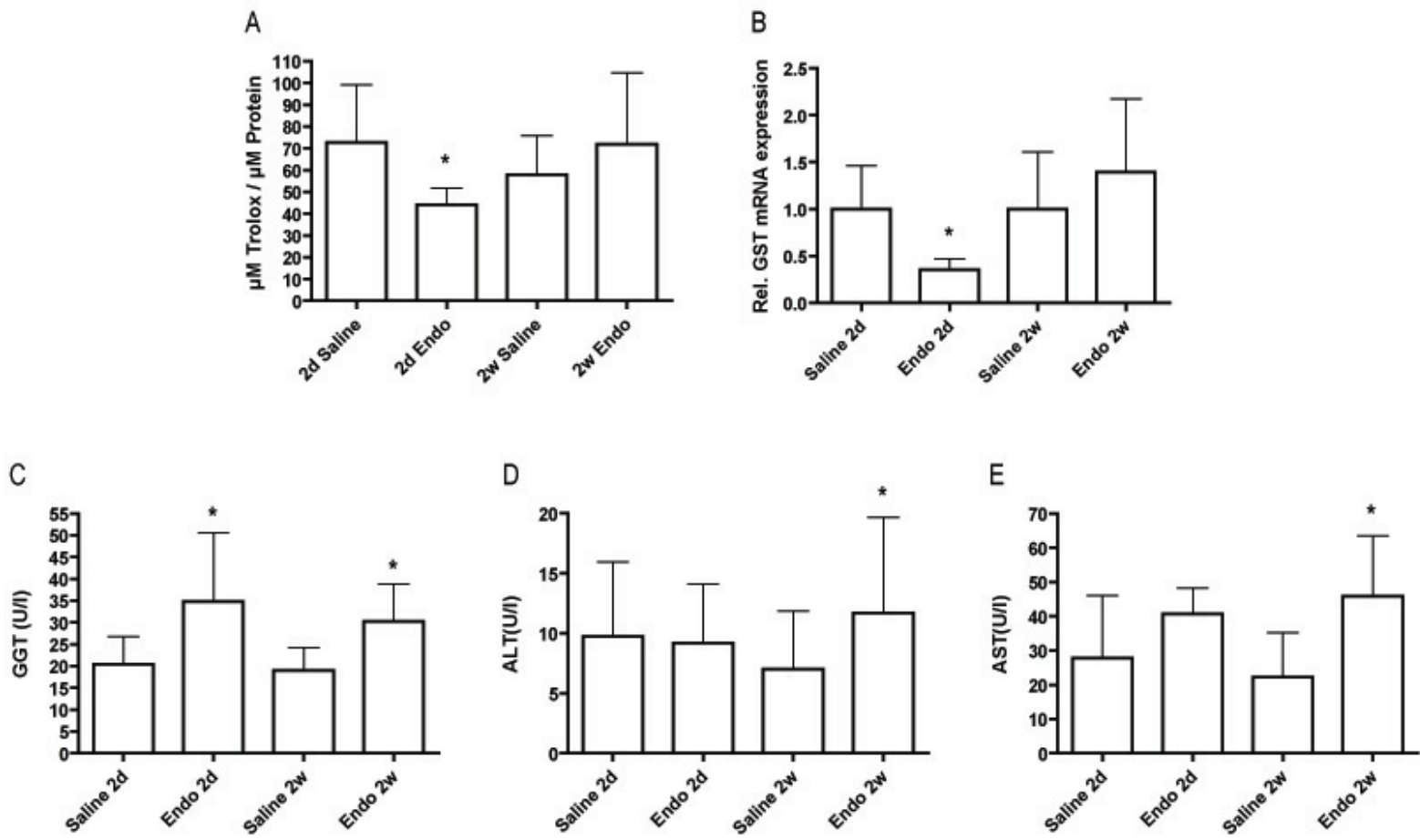

Figure 5: Quantification of oxidative stress and liver damage. TEAC assay. Overall anti-oxidant capacity expressed as $\mu \mathrm{M}$ Trolox $/ \mu \mathrm{g}$ protein (A). Relative mRNA expression of GST two days (2d) and two weeks (2w) after endotoxin (endo) compared to control animals (B). Plasma levels of GGT (C), ALT (D) and AST (E) respectively. * $=p<0.05$ Significant different from saline injected group.

\section{DISCUSSION}

The current study demonstrates for the first time that fetuses exposed to endotoxininduced chorioamnionitis develop hepatic inflammation and disturbed lipid metabolism in utero. Accordingly, our data stress the importance to monitor liver function and metabolism in infants born in the setting of chorioamnionitis-induced FIRS.

Although the liver is one of the most important organs both in fetal and in adult life, studies concerning chorioamnionitis-induced FIRS are limited to lungs, brain and gut $[84,191,192]$. In previous studies, we already investigated the inflammatory response to chorioamnionitis in lungs, brain and gut using the same fetal sheep model $[80,83]$. We have shown that endotoxin evoked a pulmonary inflammatory 
response two days after injections [80], which is not mediated by cortisol [193] and induced oxidative stress in the fetal lung $[192,194]$. The chorioamnionitis-induced inflammatory response in the brain was dose-dependent and the most prominent in the animals that received endotoxin two weeks before the delivery [83]. Comparable effects were found in the gut, where endotoxin-induced chorioamnionitis did not induce an early inflammatory response. However, two weeks after endotoxin administration preterm animals showed increased numbers of infiltrating inflammatory cells [84]. In the current study, we demonstrated for the first time that chorioamnionitis led to hepatic inflammation and disturbed fetal lipid metabolism. Although the increased inflammatory response was not associated with changes in hepatic lipid levels, plasma lipids were significantly elevated.

In liver disease, activated immune cells release inflammatory mediators such as cytokines in the micro-environment of the liver [182]. In good agreement, we observed a rapid and robust elevation of both IL- $1 \beta$ and TNF mRNA expression in the liver in response to neutrophil infiltration and CD3-positive T-lymphocyte expansion. IL-1 $\beta$ and TNF are well-known pro-inflammatory cytokines, involved in the early inflammatory response and in the evolution of the inflammatory events [195]. TNF is also one of the major activators of nuclear factor kappa $\mathrm{B}(\mathrm{NF} \kappa \mathrm{B})$, a transcription factor that plays a crucial role in inflammation and regulates the production of TNF and multiple other cytokines [196]. By promoting migration and adhesion, TNF contributes to increased infiltration of inflammatory cells, leading to a sustained inflammatory response. The observed inflammatory cell influx on H/E-, MPO- and CD3-T-lymphocyte staining, in combination with elevated cytokine expression clearly indicate that the fetal liver is inflamed in response to the endotoxininduced chorioamnionitis.

Several studies proposed that pro-inflammatory cytokines may induce meta-bolic changes [197, 198]. TNF- $\alpha$ and IL-6 could inhibit lipoprotein lipase and TNF$\alpha$ stimulates hormone-sensitive lipase and induces uncoupling protein expression. TNF- $\alpha$ can also downregulate insulin-stimulated glucose uptake and therefore evoke insulin resistance and inefficient carbohydrate conversion. Increased plasma triglyceride levels represent an early and consistent metabolic alteration during inflamma- 
tion. In addition, increased plasma total cholesterol levels and hepatic cholesterol synthesis have been reported [182]. In our study, we indeed observed a similar peak in cholesterol and triglyceride levels in combination with the hepatic inflammatory response two days after exposure to endotoxin. These changes in lipid metabolism serve as first line defense against the induced inflammation, as the effects of cytokines on lipid metabolism are direct. In cases of chronic inflammation, however, the cytokine-induced lipid changes could be deleterious and may contribute to the development of metabolic diseases [182]. Surprisingly, the early (i.e. 2 days post injection) rise in lipid levels did not persist. This unexpected observation could be attributed to the apparent ability of the fetus to modulate and downregulate lipid levels [74,199]. Although the changes in lipid levels seem to be resolved two weeks after the endotoxin injections, this issue needs further investigation, as the inflammation persisted. Indeed, as inflammation is thought to induce marked changes in lipid metabolism [182], a potential long-term effect may be the development of metabolic disease. The observed increases in plasma glucose levels after $2 \mathrm{~d}$ and $2 \mathrm{w}$ endotoxin treatment are in line with the decreases in glycogen content observed by PAS-staining. Similarly, it was shown that endotoxin treatment in mice can diminish liver glycogen content as early as $1 \mathrm{~h}$ after treatment, probably due to impairment of glycogenesis and gluconeogenesis [199].

In addition to the lipid changes and hepatic inflammation, endotoxin-induced chorioamnionitis also led to oxidative damage. Oxidative damage to tissues has been implicated in the pathogenesis of liver disease [184]; we here show evidence of immediate and sustained oxidative damage by the immunohistochemical staining against MPO. MPO is an important enzyme that is stored in azurophilic granules of polymorphonuclear (PMN) neutrophils and is therefore used as a neutrophil marker [200]. Additionally, hepatic infiltration of MPO-positive PMN neutrophils is associated with the presence of oxidized phospholipids [201]. Therefore, the presence of MPO-mediated lipid oxidation is indicative of altered lipid metabolism leading to further hepatic damage. Additional evidence for an altered redox/oxidative status in the livers of these fetuses was the decreased expression of GST two days after the endotoxin injections and the reduced capacity of the trolox assays. GST is an enzymatic anti-oxidant that is released by neutrophils and 
macrophages at sites of inflammation and its activity protects cells against oxidative stress [202,203]. The level of anti-oxidant capacity may provide a clear indication on the extent of cytotoxic damage that occurs in the liver [204]. Oxidative stress can overwhelm the anti-oxidant defense mechanisms and perturb the structure and functions of the fetal liver [202,203]. The early downregulation of GST expression we observe suggests that the fetus may have difficulties in responding to oxidative stress. Indeed, the decrease in anti-oxidant capacity in the liver of these fetuses was associated with liver damage indicated by the increase in plasma GGT levels. In addition, both plasma ALT and AST levels were increased two weeks after fetal exposure to endotoxin, indicating the presence of persisting liver damage, varying cell viability and cell membrane permeability in these fetal sheep [204].

In summary, the present study demonstrates for the first time that chorioamnionitis-induced FIRS can sensitize the fetus to develop hepatic inflammation, consequently leading to metabolic disturbances. Moreover, these metabolic changes could lead to further development of hepatic inflammation and even insulin resistance in later life [205, 206]. The underlying mechanisms of these findings and the long term effects of chorioamnionitis require further studies. However, our results already suggest an important clinical indication to monitor liver function and metabolism in chorioamnionitis-affected babies. 



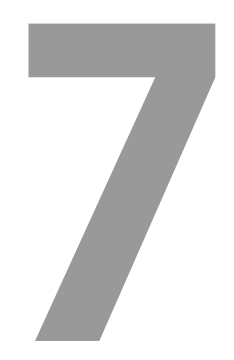

\section{Antenatal exposure to chorioamnionitis affects lipid metabolism in 7-week-old} sheep

Evi VLASSAKS, Antonio W. D. GAVILANES, Veerle BIEGHS, Andrea REINARTZ, Nikolaus GASSLER, Patrick J. VAN GORP, Marion J.J. GIJBELS, Otto BEKERS, Luc J. I. ZIMMERMANN, Jane J. PILLOW, Graeme R. POLGLASE, Ilias NITSOS, John P. NEWNHAM, Suhas G. KALLAPUR, Alan H. JOBE, Ronit SHIRI-SVERDLOV* and Boris W. KRAMER*

* These authors contributed equally to this paper.

JDOHaD 2012; 3(2):103-110 


\section{ABSTRACT}

Antenatal exposure of the fetus to inflammation may alter postnatal organ development. In previous work we demonstrated that the fetal liver is involved in the systemic inflammation associated with chorioamnionitis, leading to metabolic changes. Based on these findings, we hypothesized that chorioamnionitis can lead to postnatal inflammation-related liver injury and disturbed lipid metabolism. Chorioamnionitis was induced in sheep by intra-amniotic injection of lipopolysaccharide (LPS) or saline at 90, 100 and 110 days of gestation. Liver homeostasis and lipid metabolism were analyzed at term and at seven weeks of age. At term, hepatic T-lymphocytes and apoptotic hepatocytes were increased. Additionally, hepatic cholesterol and triglyceride levels were decreased in LPS-exposed animals compared to controls. At seven weeks of age, no hepatic inflammation could be detected. However, liver triglycerides and plasma cholesterol were increased in LPS-exposed animals relative to controls. The changes in lipid levels at seven weeks were associated with increased leptin receptor mRNA levels, increased lipid peroxidation, increased expression of cytochrome c oxidase subunit 4 (COX-4) as a marker for mitochondrial function and increased circulating ceramide levels. These findings demonstrate that chorioamnionitis-mediated antenatal inflammation-related liver disturbances have long-lasting postnatal effects on lipid metabolism. 


\section{INTRODUCTION}

Chorioamnionitis or inflammation of the amniotic fluid and fetal membranes, caused by multiple bacterial species, is the leading cause of preterm delivery and neonatal complications $[59,65]$. Even in the absence of a proven infection, fetuses and neonates may have systemic inflammation referred as the fetal inflammatory response syndrome (FIRS) [61]. Antenatal exposure to inflammation can result in adverse pulmonary, neurological and other organ development [62]. Most studies in the field of chorioamnionitis and FIRS have focused on the lungs and brain $[70,207]$. Information regarding the effects of chorioamnionitis on the liver is limited. Nevertheless, effects on the liver may play an important role in the development of metabolic diseases [88].

Previously, we demonstrated that chorioamnionitis-induced FIRS sensitizes the fetus to develop hepatic inflammation, leading to hepatocellular damage in preterm fetal sheep [169]. The livers also had disturbed glucose and lipid metabolism [169]. Numerous studies have shown that there is an association between fetal nutritional status and the risk of developing disease during later life [208]. Hence, antenatal hepatic inflammation and disturbed metabolism might trigger long-term hepatic abnormalities. We therefore hypothesized that chorioamnionitis would cause postnatal inflammation-related liver injury and disturbed lipid metabolism.

To test our hypothesis, we examined whether LPS-induced chorioamnionitis $[67,74,80-82,95,96]$ causes postnatal hepatic inflammation and lipid changes at term and at seven weeks of age. We used a model of chronic chorioamnionitis with intra-amniotic LPS (4 mg) injections at 90, 100, and 110 days gestation to mimic the physiological conditions of babies born at term after chronic chorioamnionitis exposure. We evaluated hepatic cell infiltrations, hepatocellular damage and lipid levels in liver and plasma. Further, we measured lipid storage by the measurement of mRNA expression of several genes involved in lipid metabolism, levels of sphingolipids in liver and plasma, and hepatic cytochrome c oxidase subunit 4 (COX-4) as a marker for mitochondrial function. 


\section{MATERIALS AND METHODS}

\section{Animals, intra-amniotic injections and tissue processing at delivery}

The study protocol was approved by and was performed according to the Animal Ethics Committees of the University of Western Australia (Perth, Australia) and Cincinnati Children's Hospital (Ohio, USA). The pulmonary outcome of these animals was reported previously [209]. Time-mated Merino ewes randomly received $4 \mathrm{mg}$ lipopolysaccharide (LPS - Escherichia coli 055:B5; Sigma Chemical) or the equivalent volume of saline by ultrasound guided intra-amniotic injections at 90 , 100 and 110 days gestational age (GA) [80]. All animals were kept on pasture land with the same access to food and the same environment. At 147 days GA (term gestation is 150 days), lambs were delivered surgically and humanely killed (7 LPS-exposed and 3 saline controls). A second group of lambs were allowed to deliver spontaneously at term and were humanely killed (pentobarbital $100 \mathrm{mg} / \mathrm{kg}$ ) at seven weeks postnatal age (5 LPS-exposed and 5 saline controls) (Figure 1). Blood plasma and liver were sampled and snap-frozen in liquid nitrogen for further analysis.

\section{Immunohistochemistry and histological analysis}

Inflammatory cell infiltration was identified in $7 \mu \mathrm{m}$ sections by immunohistochemical staining for T-lymphocytes (CD3) and for myeloperoxidase (MPO), predominantly synthesized by neutrophils. Endogenous peroxidase activity was blocked with $0.3 \%$ hydrogen peroxide and nonspecific binding sites were blocked with serum. After antigen retrieval (DAKO S2031, dilution 1:10), sections were incubated overnight with primary antibody (polyclonal rabbit anti-human CD3, DAKO A0452, dilution 1:200 and polyclonal rabbit anti-human MPO, DAKO A0398, dilution 1:500). Biotinylated polyclonal swine anti-rabbit IgG (DAKO E0353, dilution 1:200/1:500 respectively) was the secondary antibody. Hepatic apoptosis was determined by evaluating terminal transferase dUTP nick end labeled (TUNEL) cells using a commercially available kit (In Situ Cell Death Detection Kit, Roche Applied Science). Sections were photographed at 200x magnification using a Nikon digital camera DMX1200 and ACT-1 v2.63 software (Nikon Corporation). For the 
CD3 and MPO immunohistochemical staining, cell numbers were counted as cells per square millimetre in six randomly selected fields. TUNEL-stained sections were scored in a blinded manner by an animal pathologist (MJG). The sections from each animal were scored semi-quantitatively as follows: 0 if there were no apoptotic hepatocytes present, 1 for a few apoptotic hepatocytes ( $<3$ cell clusters), 2 for moderate cell apoptosis (3-7 cell clusters), 3 for a large number of apoptotic hepatocytes (7-9 cell clusters) and 4 if apoptosis was widespread ( $>9$ cell clusters).

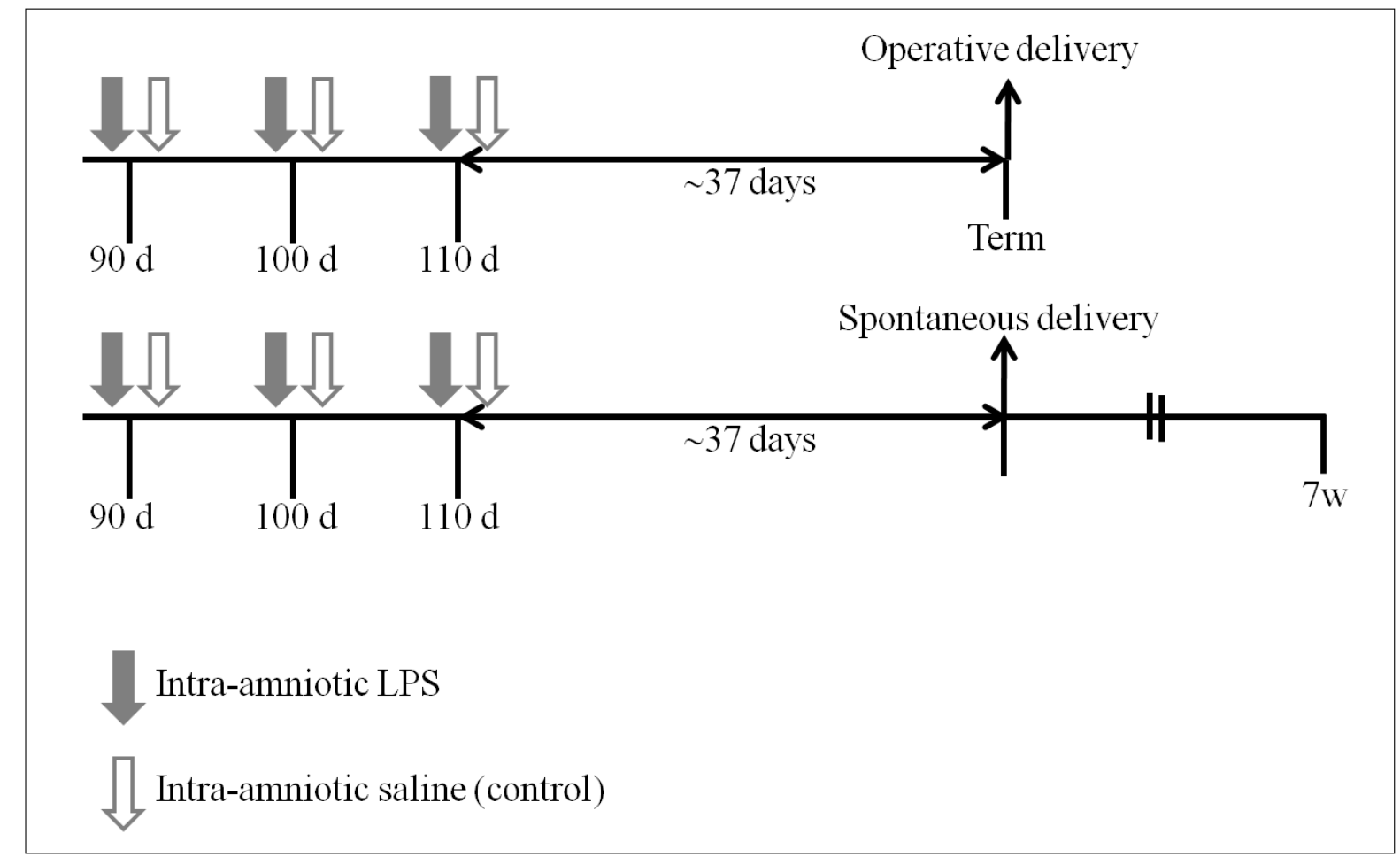

Figure 1: Experimental design. Chorioamnionitis was induced by intra-amniotic injections of LPS (saline as control) at 90, 100 and 110 days GA. Operative delivery of term fetuses was at 147 days GA (term gestation; $\mathrm{n}=3$ (control)/ $\mathrm{n}=7$ (LPS)). The remaining lambs were delivered spontaneously and were studied at seven weeks of age $(n=5 / 5)$. LPS injections are indicated as black arrows, saline injections as white arrows. 


\section{RNA extraction and RT-PCR}

Total RNA was isolated from frozen liver samples with Tri-reagent of Sigma, as described previously [89]. Reverse transcription was performed using the iScript cDNA synthesis kit (Bio-Rad) according to manufacturer's instructions. Real-time PCR was performed on a Bio-Rad MyIQ using the IQ SYBR Green Supermix with fluorescein (Bio-Rad) and $10 \mathrm{ng}$ of cDNA. Primers for target genes interleukin (IL)$1 \beta$, IL-8, tumor necrosis factor alpha (TNF $\alpha$ ), glutathione S-transferase (GST), leptin receptor, Retinoid X Receptor (RXR), and proliferator-activated receptor- $\gamma$ (PPAR $\gamma$ ) were developed using Primer Express version 2.0 (Applied Biosystems) using default settings. To standardize for the amount of cDNA, cyclophylin A (peptidylprolyl isomerase A, ppia) was used as housekeeping gene. Primer sequences are given in table 1 . The liver real-time PCR results were obtained using the relative standard curve method.

Table 1. Ovine primer sequences used in RT-PCR

\begin{tabular}{lll}
\hline Primer & Forward & Reverse \\
\hline ppia $^{\mathrm{a}}$ & TTATAAAGGTTCCTGCTTTCACAGAA & ATGGACTTGCCACCAGTACCA \\
IL-1 $\beta$ & AGAATGAGCTGTTATTTGAGGTTGATG & GTGAGAAATCTGCAGCTGGATGT \\
IL-8 & TTCCAAGCTGGCTGTTGCT & GTGGAAAGGTGTGGAATGTGTTT \\
TNF- $\alpha$ & CATCTTCTCAAGCCTCAAATAACAA & TGCGAGTAGATGAGGTAAAGCCC \\
GST & CTACATTGCCACCAAATACAACCT & AATCTGCCACCCCCTCTGA \\
RXR & CATTTTCGACAGGGTGCTG & CTTGGCGAACCTTCCTGG \\
PPAR $\gamma$ & AAAGGCGAGGGCGATCTT & CTCCCATCATTAAGGAGTTCATGTC \\
Leptin R & GATCTGTATGTAATCGTGCCAATAAT & TCCTTGTGCCCAGGAACAGT
\end{tabular}

RT-PCR, reverse transcription-PCR; ppia, peptidylprolyl isomerase A; IL, interleukin; TNF, tumor necrosis factor alpha; GST, glutathione S-transferase; RXR, retinoid X receptor; PPAR $\gamma$, proliferator-activated receptor- $\gamma$.

${ }^{\mathrm{a}}$ Housekeeping gene.

\section{Analysis of hepatic lipids}

Approximately $50 \mathrm{mg}$ of frozen liver tissue was homogenized in SET buffer (Sucrose $250 \mathrm{mM}$, EDTA $2 \mathrm{mM}$ and Tris $10 \mathrm{mM}$ ). Protein content was measured using the bicinchoninic acid method (Pierce). Triglycerides and total cholesterol were measured according to manufacturer's instructions on a Benchmark 550 Microplate reader (Bio-Rad). 


\section{Plasma parameters}

Triglycerides and total cholesterol were measured with a Beckman Coulter Synchron LX20 PRO Clinical Chemistry analyzer (Beckman Coulter). The measurements were validated in adult and fetal sheep. Alanine aminotransferase (ALT) and aspartate aminotransferase (AST) activities were determined by an enzymatic rate method on the Beckman Coulter Synchron LX20 PRO Clinical Chemistry analyzer (Beckman Coulter).

\section{Western blots}

Liver sections were homogenized in ice cold lysis buffer containing a cocktail of proteinase inhibitors and phosphatase inhibitors. 12\% SDS-PAGE gels were loaded with $25 \mu \mathrm{g}$ of total protein and transferred to a $0.45 \mu \mathrm{m}$ nitrocellulose membrane. Membranes were incubated overnight at $4{ }^{\circ} \mathrm{C}$ with monoclonal antibody to cytochrome-c oxidase subunit 4 (COX4) (Cell Signaling, dilution 1:1000). For loading control, the membranes were probed with $\beta$-actin antibody (Sigma Chemicals, dilution 1:1000).

\section{Sphingolipid analysis in liver and plasma}

Liver tissue samples $(20 \mathrm{mg}$ ) were homogenized in Aqua bidest using the UltraTurrax (ICA Labortechnik). Sphingolipids were extracted from liver homogenates or $20 \mu \mathrm{l}$ plasma (diluted 1:10 in Aqua bidest), respectively, and prepared for LC ESI-MS/MS as described previously [210], except a larger volume $(0.4 \mathrm{~mL})$ of LC mobile phase solvent was used to redissolve the final dried residue. Complex sphingolipids (Ceramide, Monohexosylceramide, Sphingomyelin, and Dihydroceramide) were separated by normal phase LC using a Supelco 2.1 (i.d.) x 50 mm LC-NH2 and a binary solvent system. Sphingoid bases and sphingoid base 1phosphates were separated by reverse phase LC using a Supelco 2.1 (i.d.) x $50 \mathrm{~mm}$ Discovery C18 column (Sigma Chemical) and a binary solvent system at a flow of $1.0 \mathrm{~mL} / \mathrm{min}$. The sphingolipids were analyzed by LC ESI-MS/MS in positive ionization mode using an ABI 3000 triple quadrupole (QQQ) tandem mass spectrometer for complex sphingolipids (Ceramide, Monohexosylceramide, Sphingomyelin, 
Dihydroceramide), and the sphingoid bases and sphingoid base 1-phosphates were analyzed using an ABI 4000 QTrap as previously described [210]. Quantitation was performed by MRM (multiple reaction monitoring) and comparison with the spiked internal standard (Sphingolipid Mix II, Avanti Polar Lipids) as well as correction for differences in ion yield due to chain length. Sphingolipid quantities are expressed as $\mathrm{pmol} / \mathrm{mg}$ protein.

\section{Data analysis}

Data were statistically analyzed using Statistical Package for Social Sciences (SPSS 17.0 Software). Comparisons between controls and LPS-injected groups were performed using two-way ANOVA controlling for age. Significant effects were analyzed using post-hoc Bonferroni corrections. Scoring of apoptotic cells was calculated by Pearson's $\chi^{2}$-test. Results are presented as mean \pm SEM and values were considered statistically significant if $\mathrm{p}<0.05$.

\section{RESULTS}

Hepatic homeostasis and lipid metabolism after LPS-induced chorioamnionitis were evaluated at term gestation and at seven weeks of age (Figure 1). The body weights of LPS-exposed lambs were not different from controls at term $(5.0 \mathrm{~kg} \pm 1.0 \mathrm{vs}$. $5.5 \mathrm{~kg} \pm 1.0)$ and at seven weeks (16.2 $\mathrm{kg} \pm 1.3$ vs. $15.5 \mathrm{~kg} \pm 1.5)$.

\section{No liver inflammation in seven week old animals}

After LPS exposure, CD3-positive T-lymphocytes increased in the livers of term animals relative to controls. The number of CD3-positive cells was increased at seven weeks relative to term animals but there was no difference between seven week old groups (Figure 2A). The numbers of MPO-positive neutrophils were not changed by LPS exposure at either age (Figure 2B). There also were no differences in the mRNA levels of IL-1 $\beta$, IL-8 and TNF between groups (table 2). 
(a)

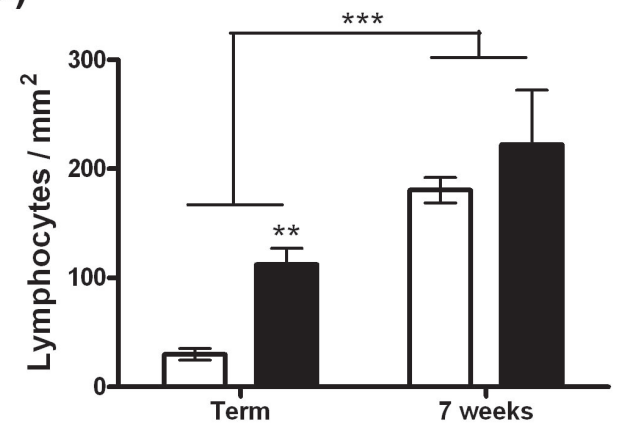

(b)

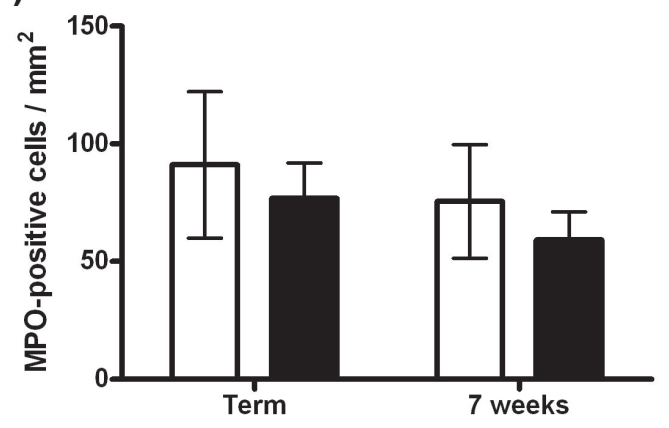

Figure 2: Hepatic inflammation based on immunohistochemistry. T-lymphocyte (CD3) staining (a) and Neutrophil (MPO) staining (b) of frozen liver sections. Results are expressed as cells $/ \mathrm{mm}^{2}$. **p $<0.01$ significantly different from saline injected group.

Table 2. Representation of IL-1 $\beta, I L-8$ and TNF $m R N A$ expression relative to cyclophylin $A$ in the four different groups

\begin{tabular}{|c|c|c|c|c|}
\hline Relative mRNA & Saline, term & LPS, term & Saline, 7 weeks & LPS, 7 weeks \\
\hline IL-1 $\beta$ & $1.00 \pm 0.27$ & $1.40 \pm 0.37$ & $1.00 \pm 0.11$ & $1.00 \pm 0.16$ \\
\hline TNF & $1.00 \pm 0.31$ & $1.40 \pm 0.44$ & $1.00 \pm 0.41$ & $0.99 \pm 0.78$ \\
\hline IL-8 & $1.00 \pm 0.26$ & $0.89 \pm 0.30$ & $1.00 \pm 0.31$ & $0.99 \pm 0.24$ \\
\hline
\end{tabular}

IL, interleukin; TNF, tumor necrosis factor alpha; LPS, lipopolysaccharide.

Values are normalized to the levels of control animals and expressed as mean \pm S.E.M.

\section{No hepatocellular damage in seven week old animals}

At term birth, TUNEL-positive hepatocytes were elevated after LPS-induced chorioamnionitis (Figure 3A). However, no changes were observed in the plasma markers for liver damage ALT and AST (data not shown). Also no indications for oxidative stress could be observed based on GST mRNA expression (Figure 3B). In the seven week old animals, no indication for liver damage was found (Figure 3A-B). 
(a)

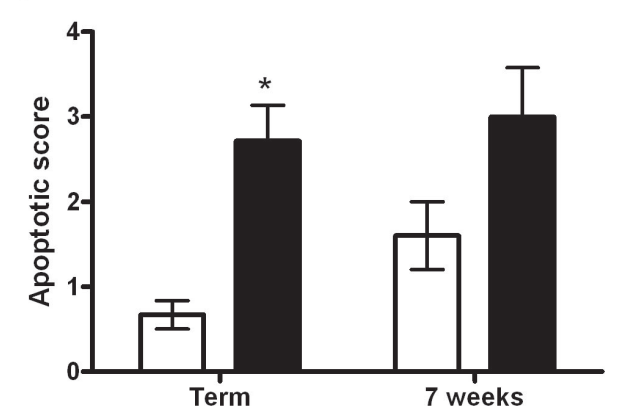

(b)

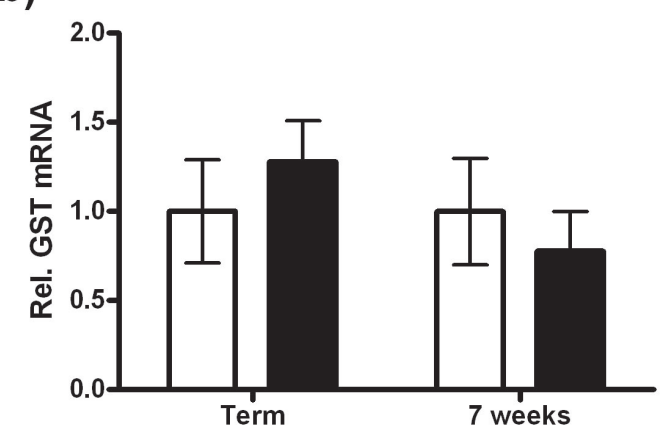

Figure 3: Quantification of hepatocellular damage. Apoptotic score of TUNELpositive cells from saline and LPS exposed animals (a). Relative mRNA expression of GST normalized to cyclophylin A (b). ${ }^{*} \mathrm{p}<0.05$ significantly different from saline injected group.

\section{Increased hepatic lipid levels in seven week old animals}

After LPS exposure, term lambs had decreased hepatic cholesterol and triglyceride levels compared to controls (Figure 4A-B). Seven weeks after birth, hepatic cholesterol levels were comparable between control and LPS-exposed animals (Figure 4A). Hepatic triglyceride levels, on the other hand, were significantly increased compared to controls in the seven week old sheep (Figure 4B). These changes in hepatic lipid levels were age-related (Figure 4A+B). In addition, both in term and in seven week old animals, plasma cholesterol levels were higher after LPSinduced chorioamnionitis (Figure 4C). Plasma triglyceride levels were not different between groups (Figure 4D). Plasma triglycerides increased in 7-week-old animals compared to term animals (Figure 4D).

\section{Increased leptin receptor mRNA levels, cytochrome c oxidase subunit}

\section{4 levels and plasma ceramides in seven week old animals}

Despite the decreased triglyceride levels, intra-amniotic LPS did not change the mRNA levels of leptin receptor, RXR and PPAR $\gamma$ in term animals (Figure 5A-C). Seven weeks after birth, however, the relative expression of leptin receptor mRNA, 
but not RXR and PPAR $\gamma$ mRNA, was higher in the LPS exposed animals compared with controls (Figure 5A-C).

(a)

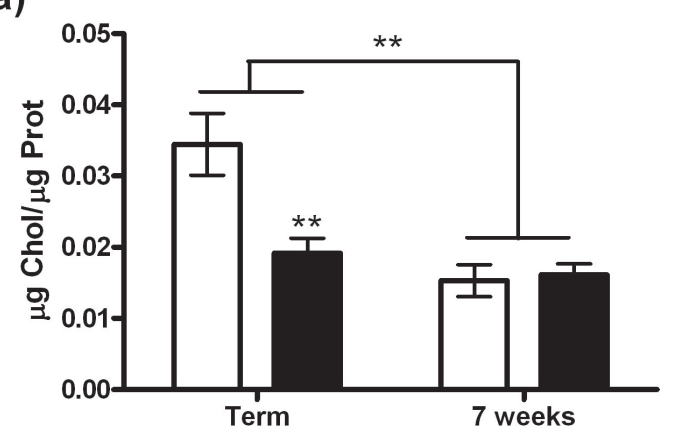

(c)

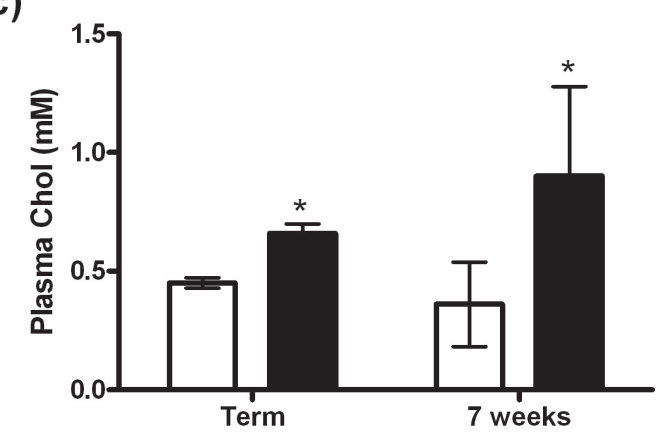

(b)

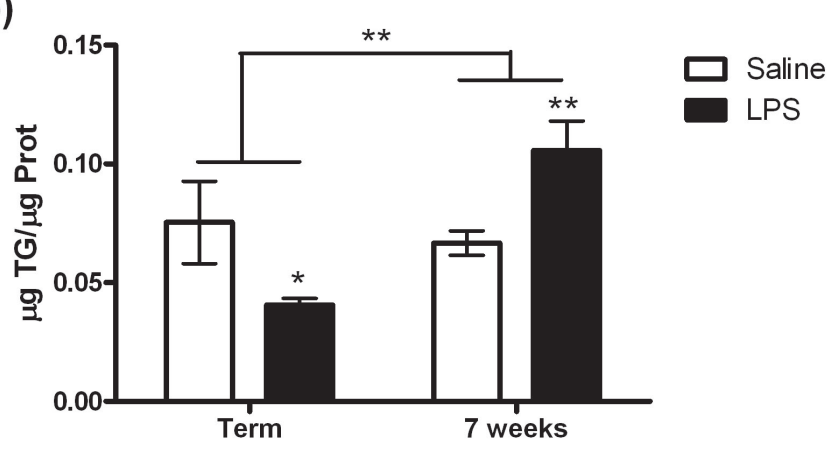

(d)

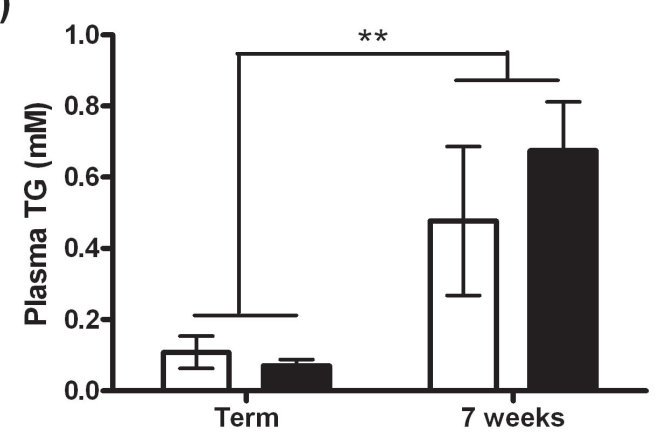

Figure 4: Liver and plasma lipid levels of saline and LPS exposed animals. Hepatic cholesterol (Chol) levels (a) and hepatic triglyceride (TG) levels (b) corrected for their protein (Prot) content. Plasma total Cholesterol (c) and plasma triglyceride (TG) levels (d). ${ }^{*} \mathrm{p}<0.05$ and $* * \mathrm{p}<0.01$ significantly different from saline injected group.

As increasing evidence demonstrates that elevated ceramide biosynthesis contributes to augmented lipid storage [211,212], we measured sphingolipids in liver and plasma. Nevertheless, there were no significant changes in hepatic ceramide metabolites for the term animals or for the seven week old animals (table 3). In plasma, however, levels of ceramide and dihydroceramide decreased after LPS exposure at birth but increased at seven weeks (table 3). Plasma sphingosine levels increased in LPS exposed sheep both at term as at seven weeks (table 3).

To investigate whether the changes in lipid levels could be due to changes in hepatic 
energy metabolism [211], we measured the expression of COX-4, a mitochondrial membrane enzyme fundamentally involved in the respiratory electron transport system and lipid oxidation [213,214]. Although we did not observe any significant changes in the term animals, Western blot analysis showed that 7-week-old LPSexposed animals had higher COX-4 protein content in liver compared to control animals (Figure 5D).

(a)

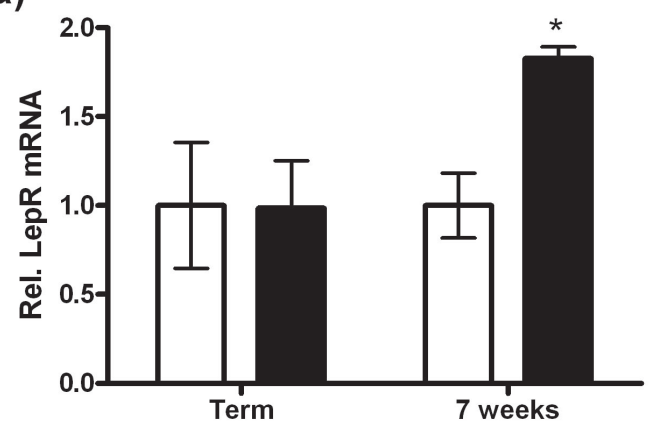

(c)

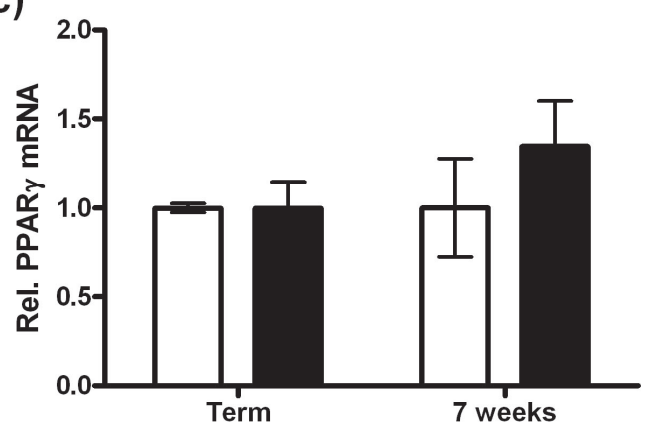

(b)

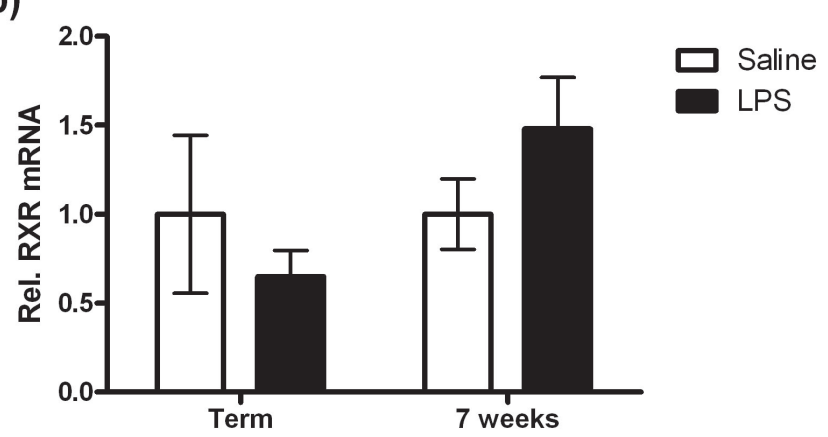

(d)

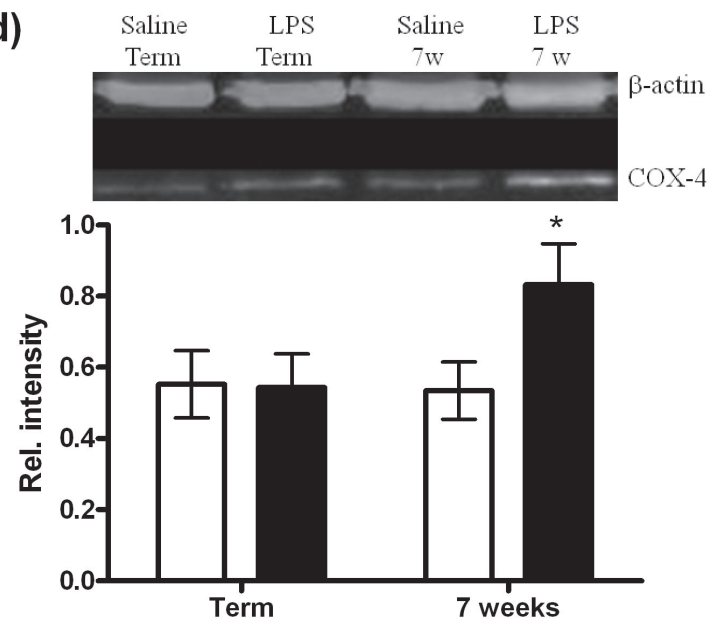

Figure 5: Relative mRNA expression level of Leptin receptor (a), RXR (b) and PPAR $\gamma(\mathrm{c})$ normalized to cyclophylin A of saline and LPS exposed animals. Representative Western Blots showing COX-4 in saline and LPS exposed animals (top) and bars representing relative band intensity of COX-4 with $\beta$-actin as loading control (bottom) (d). $* \mathrm{p}<0.05$ significantly different from saline injected group. 


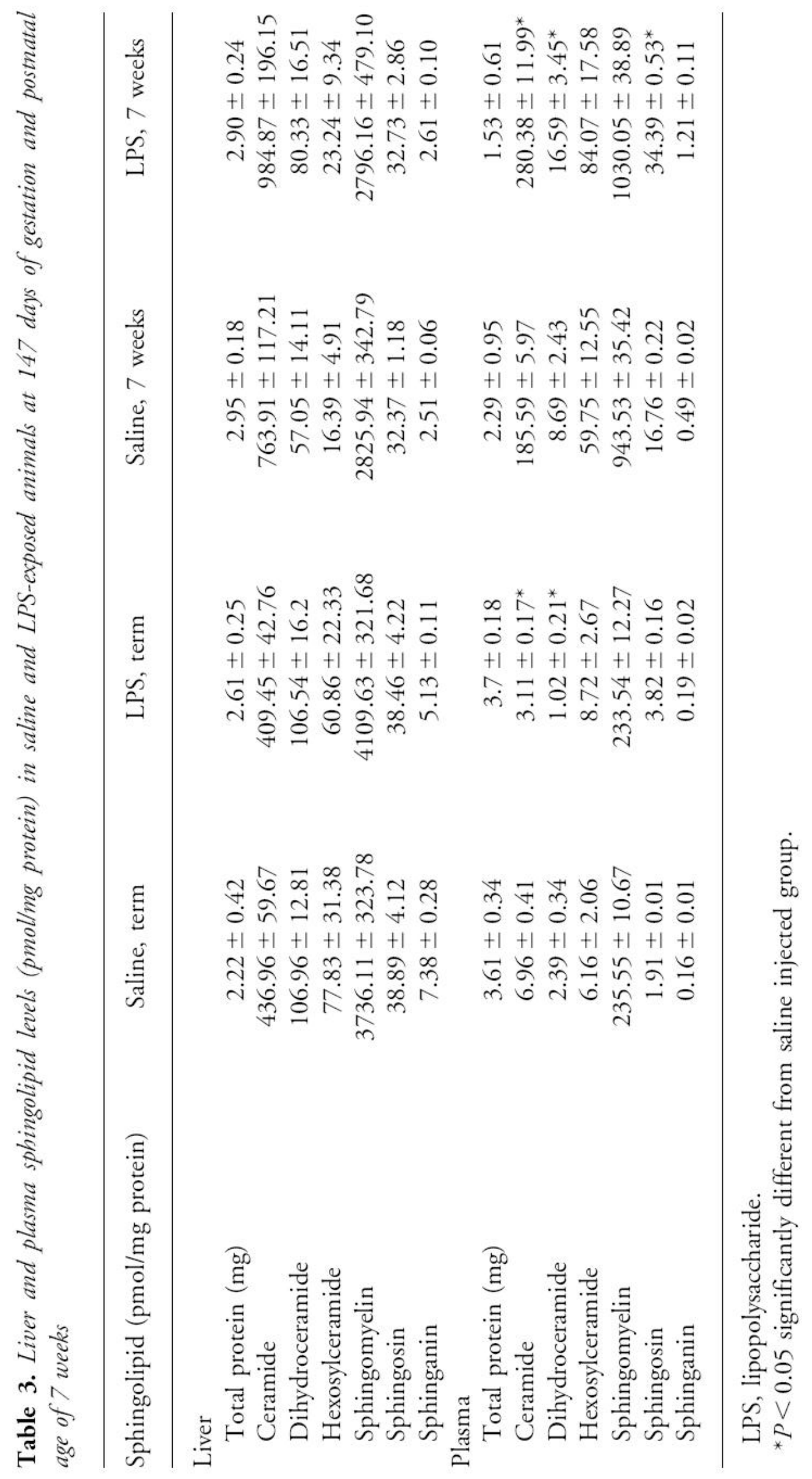




\section{DISCUSSION}

In the current study, we asked if the antenatal inflammation-related liver injury caused by LPS-induced chorioamnionitis could provoke hepatic disturbances at term and at seven weeks of age. Interestingly, the acute antenatal disturbances in lipid metabolism that were found in previous work [169], persisted for up to seven weeks. These are remarkable findings as the last antenatal LPS exposure was approximately 37 days before birth and the effect was still observed seven weeks after birth. Moreover, we found that these postnatal changes in lipid levels might be correlated with hepatic increases in lipid biosynthesis, mitochondrial biogenesis (COX-4) and circulating ceramide levels.

Fetuses exposed to chronic chorioamnionitis are at higher risk for postnatal morbidity and mortality, with the most important target organs being the brain and lungs [215]. Various animal models using intra-amniotic LPS or Ureaplasma suggest that antenatal inflammation primes the fetal lung leading to increased postnatal lung inflammation [216,217]. In addition, it has been suggested that exposure to intra-uterine inflammation might be associated with cognitive impairments [216]. However, to the best of our knowledge, the effects of chorioamnionitis on postnatal liver function have not been studied. In previous work, we demonstrated that chorioamnionitis-induced FIRS can cause hepatic inflammation, consequently leading to disturbances in glucose and lipid metabolism [169]. Therefore, fetuses exposed to chorioamnionitis may be more susceptible to develop metabolic diseases in adulthood. This might be a new twist to the Developmental Origins of Health and Disease (DOHaD) paradigm where intra-uterine metabolic conditions are correlated with outcomes in later life [218].

Although the fetal liver was inflamed shortly after fetal exposure to LPS-induced chorioamnionitis [169], the only inflammatory effect at term was increased hepatic lymphocyte infiltration. As these animals received three LPS injections that were completed 37 days before delivery, this might be an indication of chronic inflammation. However, other markers for inflammation such as the mRNA expression of pro-inflammatory cytokines were not increased at term. This is in line with the 
observation that IL-1 $\beta$ and TNF mRNA levels were not increased two weeks after LPS-induced chorioamnionitis in fetal sheep [169]. A plausible explanation could be that these cytokines are not important in chronic inflammation since they mediate particularly the early inflammatory response [195]. Further, no effect on inflammation could be observed with respect to the MPO-staining. It has been suggested that MPO-expressing cells are involved in acute as well as in chronic inflammation [200]. This chronic role of MPO was seen in the gut, where a massive influx of MPO-positive cells was observed 30 days after intra-amniotic LPS injection [84]. In addition, antenatal exposure to LPS also caused increased postnatal lung MPO levels [80,216]. Since MPO plays a postnatal role in other tissues after LPS-induced chorioamnionitis, we speculate that the postnatal liver can mitigate the inflammatory response.

The mechanisms involved in this postnatal inflammatory process may include alterations of the innate immune system [216]. The inflammatory response fully develops during the first weeks of postnatal life [219,220]. Therefore, we speculate that in the postnatal liver the immune system is maturing so that the liver can clear the inflammation.

Our current study reveals a novel finding: hepatic cholesterol and triglycerides in chorioamnionitis-exposed animals decreased at term but hepatic triglycerides and plasma cholesterol increased at the age of seven weeks. To our knowledge, nothing is known about the effects of fetal chorioamnionitis on hepatic lipid metabolism after birth. Emerging evidence indicates that inflammation and oxidative stress may be fundamental to lipid homeostasis $[197,198]$. Therefore, it is likely that the observed changes in lipid levels are triggered by the chorioamnionitis-induced FIRS. Remarkably, lipid levels did not increase but decreased after LPS exposure in term animals. This effect is also seen in growth restricted fetal sheep, due to placental insufficiency [221]. However, when these animals are then exposed to a nutrient-rich environment after birth, lipid levels increase considerably [221]. We did not manipulate or control the feeding during pregnancy. Yet, the fetal adaptation to inflammation in utero may have comparable effects on the metabolism and the subsequent adaptation postnatally. 
To gain more insights into the mechanism of increased lipid levels, we assessed whether chorioamnionitis changes the expression of genes that regulate adipogenesis (PPAR $\gamma$ and RXR) and adipokine signalling (leptin receptor). Although RXR and PPAR $\gamma$ were not significantly increased, they might have played a role as well, because the heterodimerization of these receptors induces the transcription of the leptin receptor [221]. Changes in leptin receptor were expected as this gene regulates accumulation of lipids [221] and indeed mRNA levels of leptin receptor were increased in seven week old animals exposed to prenatal chorioamnionitis.

Hepatic lipid metabolism can also be affected by impaired mitochondrial fatty acid oxidation [214]. Hence, we measured the expression of COX-4 as a mitochondrial loading control [213]. Surprisingly, COX-4 levels did not decrease after LPS exposure but instead increased in the seven week old animals. As mitochondrial lipid oxidation can reduce gene transcription and peptide synthesis [222], we speculate that the increased COX-4 levels might be a mechanism to compensate for the increased lipid accumulation due to increased leptin receptor mRNA levels.

Finally, we looked at sphingolipid levels as more and more evidence implicates ceramides to be responsible for the cytokine-induced metabolic changes [211,212, 223]. Although it is known that LPS can induce hepatic sphingolipid synthesis [224], we did not observe significant changes in hepatic ceramide metabolites in term or seven week old animals. Nevertheless, ceramides synthesized in the liver could be released into the plasma because plasma sphingolipid levels were higher after LPS exposure. Interestingly, the changes in ceramide and dihydroceramide levels correlated with the hepatic triglyceride levels supporting the idea that LPS can both induce triglyceride and cholesterol synthesis and changes in lipoproteins [223].

In summary, antenatal chorioamnionitis has consequences for postnatal hepatic metabolism. Although hepatic lipid peroxidation increased in response to the initial prenatal inflammatory provocation, hepatic triglycerides and plasma cholesterol were increased at seven weeks after birth. As these lipid changes were correlated with changes in sphingolipid levels, these animals might be more susceptible to develop further hepatic inflammation and even insulin resistance or metabolic dis- 
orders in later life $[205,206]$. However, the long term consequences in adult life of the antenatal changes seen in hepatic inflammation and metabolism remain to be established explicitly. 

Summary and General Discussion 
Inflammation is known to be a major pathological component in perinatal asphyxia and chorioamnionitis, two important birth complications possibly leading to death and life-long disabilities. Nevertheless, the underlying molecular mechanisms of these inflammatory processes and the associated pathology are not completely clear. In the present thesis, I aimed to elucidate some inflammatory mechanisms behind the pathophysiology of these neonatal complications. The major findings of the studies presented in this thesis will be described with some suggestions for further research.

\section{IMPORTANT ROLE OF INFLAMMATION DURING FETAL AND NEONATAL DEVELOPMENT}

Inflammation is a regulated process that gives a protective response to a range of harmful stimuli and is mediated by both innate and adaptive immunity. Although inflammation is necessary to limit the damage and induced regeneration of affected tissues, inappropriate or excessive regulation of the inflammatory response can lead to several human diseases [5,225].

The fetus and neonate have different capabilities to mount an inflammatory response. The gestational age is an important factor in determining the magnitude of the response and the degree of associated tissue damage [4]. The fetal immune system is known to be poorly developed in the early-/mid-gestation period and becomes functionally mature around 16-22 weeks of gestation $[4,226]$. Also the way of exposure to an inflammatory stimulus appears to impact the fetal and neonatal response [4].

The initial response at the local site of injury or bacterial invasion is associated with recruitment and activation of inflammatory cells, like endothelial cells and macrophages, which in turn produce chemokines and cytokines [5]. Cytokines are cell-signaling proteins that are secreted in response to an inflammatory or antigenic stimulus that mediate both innate and acquired immunity. The initial effects are local; however, they have type-specific, high-affinity receptors in various target organs, resulting in a variety of systemic effects. These actions include initiation of the inflammatory cascade, regulation of lymphocyte activation and chemotaxis, regulation of natural immunity, stimulation of the growth and differentation of vari- 
ous bone marrow progenitor cell lines, and regulation of immune-mediated inflammation [227]. Any dysregulation in normal expression of cytokines in the fetal circulation may affect developmental outcome [226]. To maintain homeostasis, both pro- and anti-inflammatory mediators are released [227]. If local defences are insufficient to clear the damage, inflammatory mediators are released into the systemic circulation [228]. Multiple system organ dysfunction can occur when inflammation is sustained. Finally, death results by excessive and unbalanced pro- or anti-inflammatory responses [227].

Growing body of evidence suggests that in utero exposure to infection or inflammation plays an important role in the genesis of fetal or neonatal injury leading to multi-organ morbidity $[14,229]$. Both asphyxia-induced inflammation and choriamnionitis-induced FIRS have been proposed as primary causes of tissue damage, since both induce an inflammatory reaction in the developing infant [4].

\section{INFLAMMATORY MECHANISMS AFTER FETAL AND PERINATAL ASPHYXIA}

Both human and experimental studies demonstrate an important role of cytokines in the brain response to asphyxia $[29,32,33,35,37,41,42]$. Human data revealed elevated IL-6 and TNF- $\alpha$ levels in CSF and upregulated IL-6, IL-8 and IL-10 concentrations in the sera from newborn infants with HIE compared with normal neonates $[32,34]$. Also in experimental stroke models, increased levels and activity of IL- $1 \beta$, IL- 6 and TNF- $\alpha$ several hours to days after the insult are reported $[37,38]$. In contrast, Ashdown et al. reported lower IL- $1 \beta$ and TNF- $\alpha$ protein levels and lower IL-6 mRNA levels in neonatal rat brains $2 \mathrm{~h}$ after global birth asphyxia [35]. Although these experiments indicate that cytokines may modulate asphyctic damage in brain tissue, the exact role and the time pattern of expression have not elucidated yet. Additionally, the inflammatory mechanisms behind fetal asphyctic preconditioning are not clear. It has been suggested that preconditioning ameliorates asphyxia-induced brain damage by suppressing the inflammatory response $[25,31,100]$ but no experimental studies have examined the role of cytokines after global FA preconditioning in a perinatal setting.

Therefore, in chapter 2, we aimed to elucidate the longitudinal inflammatory 
cytokine profile in response to global PA. Moreover, we investigated whether proand anti-inflammatory cytokines played a role in the induction of neuroprotection against PA injury. FA preconditioning was induced at embryonic day 17 by clamping the uterine vasculature for $30 \mathrm{~min}$. At term birth, PA was induced by placing the uterine horns, containing the pups, in a saline bath for $19 \mathrm{~min}$. At different time points after FA and PA, we assessed mRNA levels of several cytokines and related receptors in total hemispheres of fetal and neonatal brains. Consistent with our hypothesis, we demonstrated that FA preconditioning induces time-dependent cytokine changes with acute downregulation of IL- $1 \beta$, TNF- $\alpha$ and IL-10; followed by increased IL-6 mRNA levels later on. Importantly, these prenatal changes downregulate the cytokine response when combined with a global asphyctic insult at birth. PA rats show higher IL-10 and IL-6 mRNA levels than control rats. However, in FA preconditioned $(\mathrm{FA}+\mathrm{PA})$ rats this effect was attenuated, showing an inflammatory phenotype similar to control animals.

In chapter 3, we further investigated the inflammatory mechanisms behind FAinduced neuroprotection by considering the crosstalk between ceramide metabolism and the neuronal inflammatory response. Ceramides represent a major class of lipids, are essential components of eukaryotic membranes, and are involved in the regulation of cell growth and apoptosis [46]. Inflammatory cytokines can trigger changes in ceramide levels which has been reported in various models of hypoxia/ischemia $[58,141]$. We found a biphasic response to fetal asphyxia starting with an acute increase in mRNA levels of the enzymes LAG1 homolog ceramide synthase 1 (Lass1) and sphingomyelin synthase 1 (SMS1) that were correlated with decreased cytokine levels. Later in time, levels of aSMase, ceramide, sphingomyelin, galactosyl- and glycosylsylceramide were increased leading to increased levels of apoptosis and inflammatory cytokines. Even after birth, ceramide and sphingomyelin levels stayed upregulated in animals previously exposed to FA. These data indicate that FA influences the brain ceramide signaling network both acute and chronic. Moreover, a link between ceramide levels and both apoptosis and inflammation has been observed after FA. Therefore, investigation of the ceramide signaling pathway might provide important insights into the mechanisms of global asphyctic neuroprotection. 
In chapter 4 we were interested in the long-term inflammatory changes after FA and PA. Although we could not detect chronic changes in inflammatory cytokines, the data showed that FA and PA have region-specific, persisting effects on mRNA levels of ceramide genes. The data also suggest that the combination of two subsequent asphyctic insults provides long-lasting neuroprotection in the prefrontal cortex probably by maintaining normal apoptosis and promoting cell proliferation.

Finally, as part of the systemic impact of asphyxia, we investigated in chapter 5 the acute and chronic hepatic inflammatory response after both FA and PA. FA induced an acute increase in IL- $1 \beta$ followed by increased IL- 6 mRNA levels. This inflammatory response was associated with increased mRNA levels of Lass 1 and ceramide transporters CERT and GPBP. At $96 \mathrm{~h}$ after FA, aSMase and SMS1 mRNA levels increased together with less induction of BAD and oxidative DNA damage. Also after PA an inflammatory response was observed, with increased IL-6 and IL-10 mRNA levels $2 \mathrm{~h}$ after birth. The combination of FA and PA (preconditioning) attenuated the inflammatory response, reflecting comparable IL-6 and IL-10 mRNA levels as control animals. Interestingly, the protective effect of preconditioning was also observed when considering apoptotic genes. Although no clear differences in liver structure or hepatocellular damage could be observed after FA or PA, animals that experienced both FA and PA during birth showed increased plasma levels of alkaline phosphatase at eight months of age. Therefore, more studies are needed to investigate the long-term hepatic changes after perinatal asphyxia.

In summary, our findings provide new insights into the neuroprotective mechanism of fetal asphyxia. We suggest that inflammatory cytokines may be the underlying cause of PA-induced brain damage. Downregulating this cytokine synthesis might be neuroprotective since FA-preconditioned animals show cytokine levels comparable to control levels. Interestingly, cytokine levels seem to modulate the expression pattern of sphingolipids, important components of the cell membrane that regulate cell differentiation and apoptosis [48]. This raises the possibility that ceramides, in response to cytokine production, can modulate the inflammatory response to an asphyctic insult. More research is needed to fully characterize the action mechanism 
of cytokines and ceramides in asphyxia-mediated injury. Moreover, it is not clear which mechanisms initiate inflammatory injury to the asphyctic brain.

It is now recognized that mitochondrial dysfunction during asphyxia and the generation of mitochondrial reactive oxygen species (ROS) during reperfusion play a major role in the cellular damage [230]. In mature animal models of hypoxiaischemia, the mitochondrial respiratory chain has been shown to be an important source for the reperfusion-driven generation of ROS responsible for oxidative injury [230]. Its exact role and its spatial-temporal relationship with inflammation are elusive but provide a nice target for further research.

\section{HEPATIC INFLAMMATORY MECHANISMS AFTER CHORIOAMNIONITIS}

Leviton and Adinolfi first proposed that a maternal infection, such as chorioamnionitis, can lead to elevated fetal inflammation, which might result in fetal disease and preterm birth [231,232]. Gomez et al. have broaden this concept and shown that the fetal response is the most important predictor of the infants' outcome; these researchers coined the fetal inflammatory response syndrome (FIRS) concept [233]. Nowadays, the mechanisms whereby chorioamnionitis induces FIRS are still poorly understood. Much research in the field of FIRS has focussed on the lungs and brain [61]. Various animal models using intra-amniotic endotoxin or Ureaplasma injection suggest that antenatal inflammation primes the fetal lung leading to exacerbated postnatal lung inflammation [216,217]. In addition, it has been suggested that exposure to intrauterine infection might be associated with cognitive limitations [216]. However, the effects of chorioamnionitis on the liver have never been reported.

Accordingly, in chapter 6, we hypothesized that chorioamnionitis induces an inflammatory response in the fetal liver, which would lead to fetal metabolic disturbances. Using a sheep model for chorioamnionitis, we found that two days after the endotoxin injections, inflammatory markers were significantly higher compared to controls. Additionally, lipid and glucose metabolism were disturbed in response to endotoxin. Moreover, the anti-oxidant state capacity was reduced and hepatic damage was apparent. Two weeks after the endotoxin injections the fetal livers 
were still inflamed and had higher glucose concentrations in the blood. In addition, the levels of markers for hepatic damage (ALT, AST) were elevated. These results indicated that chorioamnionitis induces liver inflammation, leading to metabolic disturbances in the fetus.

In chapter 7, we investigated if chorioamnionitis can also lead to postnatal inflammation-related liver injury and disturbed lipid metabolism. At term, hepatic T-lymphocytes and apoptotic hepatocytes were increased. Additionally, hepatic cholesterol and triglyceride levels were decreased in LPS-exposed animals compared to controls. At seven weeks of age, no hepatic inflammation could be detected. However, liver triglycerides and plasma cholesterol were increased in LPSexposed animals relative to controls. The changes in lipid levels at seven weeks were associated with increased leptin receptor mRNA levels, increased lipid peroxidation, increased expression of cytochrome c oxidase subunit 4 (COX-4) as a marker for mitochondrial function and increased circulating ceramide levels. These findings demonstrate that chorioamnionitis-mediated antenatal inflammation-related liver disturbances have long-lasting postnatal effects on lipid metabolism.

Altogether, these results indicate that antenatal inflammation induced by chorioamnionitis affects the liver both pre- and postnatally. Moreover, glucose and lipid metabolism are influenced possibly priming the fetus/newborn to develop metabolic diseases in later life. These are the first studies indicating an important role of chorioamnionitis in the development of hepatic disease and give a new twist to the Developmental Origin of Health and Disease paradigm. Despite increasing knowledge in the inflammatory mechanisms involved in chorioamnionitis, the central question remains how the naive innate immune system of the fetus actively controls the inflammation and injury [62]. Since the liver has indispensable functions in modulating inflammation, more research in this direction is needed to identify therapeutic targets in suitable animal models [234]. 



\section{Bibliography}

[1] E. V. Wachtel and K. D. Hendricks-Munoz, "Current management of the infant who presents with neonatal encephalopathy," Curr Probl Pediatr Adolesc Health Care, vol. 41, pp. 132-153, 2011.

[2] R. Bracci, S. Perrone, and G. Buonocore, "The timing of neonatal brain damage," Biol. Neonate, vol. 90, pp. 145-155, 2006.

[3] S. Girard, H. Kadhim, M. Roy, K. Lavoie, M. E. Brochu, A. Larouche, and G. Sebire, "Role of perinatal inflammation in cerebral palsy," Pediatr. Neurol., vol. 40, pp. 168-174, Mar 2009.

[4] P. Rezaie and A. Dean, "Periventricular leukomalacia, inflammation and white matter lesions within the developing nervous system," Neuropathology, vol. 22, pp. 106-132, Sep 2002.

[5] M. W. Kemp, M. Saito, J. P. Newnham, I. Nitsos, K. Okamura, and S. G. Kallapur, "Preterm birth, infection, and inflammation advances from the study of animal models," Reprod Sci, vol. 17, pp. 619-628, Jul 2010.

[6] J. J. Volpe, "Perinatal brain injury: from pathogenesis to neuroprotection," Ment Retard Dev Disabil Res Rev, vol. 7, pp. 56-64, 2001.

[7] R. E. Black, S. Cousens, H. L. Johnson, J. E. Lawn, I. Rudan, D. G. Bassani, P. Jha, H. Campbell, C. F. Walker, R. Cibulskis, T. Eisele, L. Liu, C. Mathers, R. E. Black, J. Bryce, L. Caulfield, C. F. Walker, H. Johnson, H. Kalter, J. Katz, L. Liu, N. Walker, D. Bassani, P. Jha, Z. Bhutta, T. Eisele, H. Campbell, I. Rudan, E. Theodoratou, S. Cousens, V. Filippi, M. Ezzati, C. Lanata, J. Lawn, H. Peterson, R. Steketee, R. Bahl, T. Boerma, T. Cherian, O. Fontaine, F. Gore, R. Hutubessy, J. Martines, L. Muhe, S. Qazi, D. Brown, M. Young, M. Chopra, M. Oestergaard, M. Inoue, D. M. Fat, V. Joseph, C. B. Pinto, N. Binkin, C. Weidemann, K. Chan, Y. Guo, R. Kumar, S. Awasthi, A. Adler, S. Oza, M. Aryee, and L. Lamberti, "Global, regional, and national causes of child mortality in 2008: a systematic analysis," Lancet, vol. 375, pp. 1969-1987, Jun 2010. 
[8] J. A. Low, "Determining the contribution of asphyxia to brain damage in the neonate," J. Obstet. Gynaecol. Res., vol. 30, pp. 276-286, Aug 2004.

[9] R. H. Pfister and R. F. Soll, "Hypothermia for the treatment of infants with hypoxic-ischemic encephalopathy," J Perinatol, vol. 30 Suppl, pp. S82-87, Oct 2010.

[10] J. A. Westgate, A. J. Gunn, and T. R. Gunn, "Antecedents of neonatal encephalopathy with fetal acidaemia at term," Br J Obstet Gynaecol, vol. 106, pp. 774-782, Aug 1999.

[11] S. Rees, R. Harding, and D. Walker, "The biological basis of injury and neuroprotection in the fetal and neonatal brain," Int. J. Dev. Neurosci., vol. 29, pp. 551-563, Oct 2011.

[12] A. Tarcan, F. Tiker, H. Guvenir, and B. Gurakan, "Hepatic involvement in perinatal asphyxia," J. Matern. Fetal. Neonatal. Med., vol. 20, pp. 407-410, May 2007.

[13] M. de Haan, J. S. Wyatt, S. Roth, F. Vargha-Khadem, D. Gadian, and M. Mishkin, "Brain and cognitive-behavioural development after asphyxia at term birth," Dev Sci, vol. 9, pp. 350-358, Jul 2006.

[14] A. Jensen, Y. Garnier, J. Middelanis, and R. Berger, "Perinatal brain damage-from pathophysiology to prevention," Eur. J. Obstet. Gynecol. Reprod. Biol., vol. 110 Suppl 1, pp. S70-79, Sep 2003.

[15] J. Armstrong-Wells, T. J. Bernard, R. Boada, and M. Manco-Johnson, "Neurocognitive outcomes following neonatal encephalopathy," NeuroRehabilitation, vol. 26, pp. 27-33, 2010.

[16] L. S. de Vries and F. M. Cowan, "Evolving understanding of hypoxicischemic encephalopathy in the term infant," Semin Pediatr Neurol, vol. 16, pp. 216-225, Dec 2009.

[17] H. H. de Haan and T. H. Hasaart, "Neuronal death after perinatal asphyxia," Eur. J. Obstet. Gynecol. Reprod. Biol., vol. 61, pp. 123-127, Aug 1995. 
[18] P. P. Drury, L. Bennet, and A. J. Gunn, "Mechanisms of hypothermic neuroprotection," Semin Fetal Neonatal Med, vol. 15, pp. 287-292, Oct 2010.

[19] J. M. Perlman, "Intervention strategies for neonatal hypoxic-ischemic cerebral injury," Clin Ther, vol. 28, pp. 1353-1365, Sep 2006.

[20] H. Hagberg, O. Dammann, C. Mallard, and A. Leviton, "Preconditioning and the developing brain," Semin. Perinatol., vol. 28, pp. 389-395, Dec 2004.

[21] J. M. Gidday, "Cerebral preconditioning and ischaemic tolerance," Nat. Rev. Neurosci., vol. 7, pp. 437-448, Jun 2006.

[22] U. Dirnagl, R. P. Simon, and J. M. Hallenbeck, "Ischemic tolerance and endogenous neuroprotection," Trends Neurosci., vol. 26, pp. 248-254, May 2003.

[23] C. Mallard and H. Hagberg, "Inflammation-induced preconditioning in the immature brain," Semin Fetal Neonatal Med, vol. 12, pp. 280-286, Aug 2007.

[24] N. M. Jones, L. Kardashyan, J. K. Callaway, E. M. Lee, and P. M. Beart, "Long-term functional and protective actions of preconditioning with hypoxia, cobalt chloride, and desferrioxamine against hypoxic-ischemic injury in neonatal rats," Pediatr. Res., vol. 63, pp. 620-624, Jun 2008.

[25] J. A. Shin, E. M. Park, J. S. Choi, S. M. Seo, J. L. Kang, K. E. Lee, and S. Cho, "Ischemic preconditioning-induced neuroprotection is associated with differential expression of IL-1beta and IL-1 receptor antagonist in the ischemic cortex,” J. Neuroimmunol., vol. 217, pp. 14-19, Dec 2009.

[26] R. C. Vannucci, J. Towfighi, and S. J. Vannucci, "Hypoxic preconditioning and hypoxic-ischemic brain damage in the immature rat: pathologic and metabolic correlates," J. Neurochem., vol. 71, pp. 1215-1220, Sep 1998. 
[27] E. Strackx, D. L. Van den Hove, J. Prickaerts, L. Zimmermann, H. W. Steinbusch, C. E. Blanco, A. W. Gavilanes, and J. S. Vles, "Fetal asphyctic preconditioning protects against perinatal asphyxia-induced behavioral consequences in adulthood," Behav. Brain Res., vol. 208, pp. 343-351, Apr 2010.

[28] E. Strackx, B. Zoer, D. Van den Hove, H. Steinbusch, H. Steinbusch, C. Blanco, J. S. Vles, E. Villamor, and A. W. Gavilanes, "Brain apoptosis and carotid artery reactivity in fetal asphyctic preconditioning," Front Biosci (Schol Ed), vol. 2, pp. 781-790, 2010.

[29] F. S. Silverstein, J. D. Barks, P. Hagan, X. H. Liu, J. Ivacko, and J. Szaflarski, "Cytokines and perinatal brain injury," Neurochem. Int., vol. 30, pp. 375383, 1997.

[30] A. Denes, P. Thornton, N. J. Rothwell, and S. M. Allan, "Inflammation and brain injury: acute cerebral ischaemia, peripheral and central inflammation," Brain Behav. Immun., vol. 24, pp. 708-723, Jul 2010.

[31] W. Yin, A. P. Signore, M. Iwai, G. Cao, Y. Gao, M. J. Johnnides, R. W. Hickey, and J. Chen, "Preconditioning suppresses inflammation in neonatal hypoxic ischemia via Akt activation," Stroke, vol. 38, pp. 1017-1024, Mar 2007.

[32] R. C. Silveira and R. S. Procianoy, "Interleukin-6 and tumor necrosis factoralpha levels in plasma and cerebrospinal fluid of term newborn infants with hypoxic-ischemic encephalopathy," J. Pediatr., vol. 143, pp. 625-629, Nov 2003.

[33] H. Aly, M. T. Khashaba, M. El-Ayouty, O. El-Sayed, and B. M. Hasanein, "IL-1beta, IL-6 and TNF-alpha and outcomes of neonatal hypoxic ischemic encephalopathy," Brain Dev., vol. 28, pp. 178-182, Apr 2006.

[34] K. Okazaki, A. Nishida, M. Kato, K. Kozawa, N. Uga, and H. Kimura, "Elevation of cytokine concentrations in asphyxiated neonates," Biol. Neonate, vol. 89, no. 3, pp. 183-189, 2006. 
[35] H. Ashdown, S. Joita, G. N. Luheshi, and P. Boksa, "Acute brain cytokine responses after global birth hypoxia in the rat," J. Neurosci. Res., vol. 86, pp. 3401-3409, Nov 2008.

[36] R. C. Vannucci and S. J. Vannucci, "A model of perinatal hypoxic-ischemic brain damage," Ann. N. Y. Acad. Sci., vol. 835, pp. 234-249, Dec 1997.

[37] H. Hagberg, E. Gilland, E. Bona, L. A. Hanson, M. Hahin-Zoric, M. Blennow, M. Holst, A. McRae, and O. Soder, "Enhanced expression of interleukin (IL)-1 and IL-6 messenger RNA and bioactive protein after hypoxia-ischemia in neonatal rats," Pediatr. Res., vol. 40, pp. 603-609, Oct 1996.

[38] J. Szaflarski, D. Burtrum, and F. S. Silverstein, "Cerebral hypoxia-ischemia stimulates cytokine gene expression in perinatal rats," Stroke, vol. 26, pp. 1093-1100, Jun 1995.

[39] C. C. Chao, S. Hu, L. Ehrlich, and P. K. Peterson, "Interleukin-1 and tumor necrosis factor-alpha synergistically mediate neurotoxicity: involvement of nitric oxide and of N-methyl-D-aspartate receptors," Brain Behav. Immun., vol. 9, pp. 355-365, Dec 1995.

[40] A. S. Haqqani, M. Nesic, E. Preston, E. Baumann, J. Kelly, and D. Stanimirovic, "Characterization of vascular protein expression patterns in cerebral ischemia/reperfusion using laser capture microdissection and ICATnanoLC-MS/MS," FASEB J., vol. 19, pp. 1809-1821, Nov 2005.

[41] E. Bona, A. L. Andersson, K. Blomgren, E. Gilland, M. Puka-Sundvall, K. Gustafson, and H. Hagberg, "Chemokine and inflammatory cell response to hypoxia-ischemia in immature rats," Pediatr. Res., vol. 45, pp. 500-509, Apr 1999.

[42] A. Martin-Ancel, A. Garcia-Alix, D. Pascual-Salcedo, F. Cabanas, M. Valcarce, and J. Quero, "Interleukin-6 in the cerebrospinal fluid after perinatal asphyxia is related to early and late neurological manifestations," Pediatrics, vol. 100, pp. 789-794, Nov 1997. 
[43] A. J. Bruce, W. Boling, M. S. Kindy, J. Peschon, P. J. Kraemer, M. K. Carpenter, F. W. Holtsberg, and M. P. Mattson, "Altered neuronal and microglial responses to excitotoxic and ischemic brain injury in mice lacking TNF receptors," Nat. Med., vol. 2, pp. 788-794, Jul 1996.

[44] S. A. Loddick, A. V. Turnbull, and N. J. Rothwell, "Cerebral interleukin-6 is neuroprotective during permanent focal cerebral ischemia in the rat," $J$. Cereb. Blood Flow Metab., vol. 18, pp. 176-179, Feb 1998.

[45] R. M. Adibhatla, R. Dempsy, and J. F. Hatcher, "Integration of cytokine biology and lipid metabolism in stroke," Front. Biosci., vol. 13, pp. 12501270, 2008.

[46] A. Gomez-Munoz, "Ceramide 1-phosphate/ceramide, a switch between life and death," Biochim. Biophys. Acta, vol. 1758, pp. 2049-2056, Dec 2006.

[47] S. A. Novgorodov and T. I. Gudz, "Ceramide and mitochondria in ischemia/reperfusion," J. Cardiovasc. Pharmacol., vol. 53, pp. 198-208, Mar 2009.

[48] C. Mencarelli, M. Losen, C. Hammels, J. De Vry, M. K. Hesselink, H. W. Steinbusch, M. H. De Baets, and P. Martinez-Martinez, "The ceramide transporter and the Goodpasture antigen binding protein: one protein-one function?," J. Neurochem., vol. 113, pp. 1369-1386, Jun 2010.

[49] T. A. Taha, T. D. Mullen, and L. M. Obeid, "A house divided: ceramide, sphingosine, and sphingosine-1-phosphate in programmed cell death," Biochim. Biophys. Acta, vol. 1758, pp. 2027-2036, Dec 2006.

[50] R. G. Cutler and M. P. Mattson, "Sphingomyelin and ceramide as regulators of development and lifespan," Mech. Ageing Dev., vol. 122, pp. 895-908, Jul 2001.

[51] C. Mao and L. M. Obeid, "Ceramidases: regulators of cellular responses mediated by ceramide, sphingosine, and sphingosine-1-phosphate," Biochim. Biophys. Acta, vol. 1781, pp. 424-434, Sep 2008. 
[52] S. J. Gilbert, E. J. Blain, P. Jones, V. C. Duance, and D. J. Mason, "Exogenous sphingomyelinase increases collagen and sulphated glycosaminoglycan production by primary articular chondrocytes: an in vitro study," Arthritis Res. Ther., vol. 8, no. 4, p. R89, 2006.

[53] X. Liu, Y. H. Zeidan, S. Elojeimy, D. H. Holman, A. M. El-Zawahry, G. W. Guo, A. Bielawska, J. Bielawski, Z. Szulc, S. Rubinchik, J. Y. Dong, T. E. Keane, M. Tavassoli, Y. A. Hannun, and J. S. Norris, "Involvement of sphingolipids in apoptin-induced cell killing," Mol. Ther., vol. 14, pp. 627-636, Nov 2006.

[54] Z. F. Yu, M. Nikolova-Karakashian, D. Zhou, G. Cheng, E. H. Schuchman, and M. P. Mattson, "Pivotal role for acidic sphingomyelinase in cerebral ischemia-induced ceramide and cytokine production, and neuronal apoptosis," J. Mol. Neurosci., vol. 15, pp. 85-97, Oct 2000.

[55] Y. Feng and M. H. LeBlanc, "N-tosyl-L-phenylalanyl-chloromethyl ketone reduces ceramide during hypoxic-ischemic brain injury in newborn rat," Eur. J. Pharmacol., vol. 551, pp. 34-40, Dec 2006.

[56] J. Cui, R. M. Engelman, N. Maulik, and D. K. Das, "Role of ceramide in ischemic preconditioning," J. Am. Coll. Surg., vol. 198, pp. 770-777, May 2004.

[57] M. I. Bhuiyan, M. N. Islam, S. Y. Jung, H. H. Yoo, Y. S. Lee, and C. Jin, "Involvement of ceramide in ischemic tolerance induced by preconditioning with sublethal oxygen-glucose deprivation in primary cultured cortical neurons of rats," Biol. Pharm. Bull., vol. 33, pp. 11-17, Jan 2010.

[58] J. Liu, I. Ginis, M. Spatz, and J. M. Hallenbeck, "Hypoxic preconditioning protects cultured neurons against hypoxic stress via TNF-alpha and ceramide," Am. J. Physiol., Cell Physiol., vol. 278, pp. C144-153, Jan 2000.

[59] R. L. Goldenberg, J. C. Hauth, and W. W. Andrews, "Intrauterine infection and preterm delivery," N. Engl. J. Med., vol. 342, pp. 1500-1507, May 2000. 
[60] J. H. Steel, S. Malatos, N. Kennea, A. D. Edwards, L. Miles, P. Duggan, P. R. Reynolds, R. G. Feldman, and M. H. Sullivan, "Bacteria and inflammatory cells in fetal membranes do not always cause preterm labor," Pediatr. Res., vol. 57, pp. 404-411, Mar 2005.

[61] F. Gotsch, R. Romero, J. P. Kusanovic, S. Mazaki-Tovi, B. L. Pineles, O. Erez, J. Espinoza, and S. S. Hassan, "The fetal inflammatory response syndrome," Clin Obstet Gynecol, vol. 50, pp. 652-683, Sep 2007.

[62] M. Gantert, J. V. Been, A. W. Gavilanes, Y. Garnier, L. J. Zimmermann, and B. W. Kramer, "Chorioamnionitis: a multiorgan disease of the fetus?," $J$ Perinatol, vol. 30 Suppl, pp. 21-30, Oct 2010.

[63] H. L. Halliday, "Evidence-based neonatal care," Best Pract Res Clin Obstet Gynaecol, vol. 19, pp. 155-166, Feb 2005.

[64] M. Hallman, "Cytokines, pulmonary surfactant and consequences of intrauterine infection," Biol. Neonate, vol. 76 Suppl 1, pp. 2-9, Jun 1999.

[65] R. Romero, J. Espinoza, T. Chaiworapongsa, and K. Kalache, "Infection and prematurity and the role of preventive strategies," Semin Neonatol, vol. 7, pp. 259-274, Aug 2002.

[66] R. Behrman and A. Butler, Preterm birth: causes, consequences, and prevention. The National Academies Press, Washington, D.C., 2007.

[67] B. W. Kramer, U. Kaemmerer, M. Kapp, D. Herbst, A. Marx, D. Berg, P. A. Groneck, and C. P. Speer, "Decreased expression of angiogenic factors in placentas with chorioamnionitis after preterm birth," Pediatr. Res., vol. 58, pp. 607-612, Sep 2005.

[68] O. M. Faye-Petersen, "The placenta in preterm birth," J. Clin. Pathol., vol. 61, pp. 1261-1275, Dec 2008.

[69] A. B. Onderdonk, J. L. Hecht, T. F. McElrath, M. L. Delaney, E. N. Allred, A. Leviton, B. Shah, S. Pflueger, G. Markenson, C. R. Martin, J. L. Hecht, 
B. Cohen, L. J. Van Marter, T. McElrath, A. Leviton, R. Insoft, D. Roberts, L. Riley, C. Cole, J. Fiascone, I. Bhan, S. Craigo, T. Marino, F. Bednarek, G. Ayata, E. Delpapa, R. Ehrenkranz, M. Reyes-Mugica, E. Zambrano, K. P. Williams, T. M. O'Shea, D. W. Ross, M. Harper, S. Engelke, J. Christie, H. Hadi, C. Bose, C. Livasy, K. Boggess, M. Portenga, B. Doss, C. Cook, P. Karna, G. Chamyan, S. Roth, M. D. Schreiber, A. Husain, M. Ismail, D. Batton, C. H. Chang, R. Lorenz, H. Kliman, and P. Senagore, "Colonization of second-trimester placenta parenchyma," Am. J. Obstet. Gynecol., vol. 199, pp. 1-52, Jul 2008.

[70] R. Romero, J. Espinoza, L. F. Goncalves, J. P. Kusanovic, L. Friel, and S. Hassan, "The role of inflammation and infection in preterm birth," Semin. Reprod. Med., vol. 25, pp. 21-39, Jan 2007.

[71] M. A. Elovitz and C. Mrinalini, "Animal models of preterm birth," Trends Endocrinol. Metab., vol. 15, pp. 479-487, Dec 2004.

[72] J. O. Fahey, "Clinical management of intra-amniotic infection and chorioamnionitis: a review of the literature," J Midwifery Womens Health, vol. 53, pp. 227-235, 2008.

[73] J. R. Duncan, M. L. Cock, J. P. Scheerlinck, K. T. Westcott, C. McLean, R. Harding, and S. M. Rees, "White matter injury after repeated endotoxin exposure in the preterm ovine fetus," Pediatr. Res., vol. 52, pp. 941-949, Dec 2002.

[74] B. W. Kramer and A. H. Jobe, "The clever fetus: responding to inflammation to minimize lung injury," Biol. Neonate, vol. 88, pp. 202-207, 2005.

[75] M. J. Czikk, F. P. McCarthy, and K. E. Murphy, "Chorioamnionitis: from pathogenesis to treatment," Clin. Microbiol. Infect., vol. 17, pp. 1304-1311, Sep 2011.

[76] S. G. Fishman and S. E. Gelber, "Evidence for the clinical management of chorioamnionitis," Semin Fetal Neonatal Med, vol. 17, pp. 46-50, Feb 2012. 
[77] J. V. Been, P. L. Degraeuwe, B. W. Kramer, and L. J. Zimmermann, "Antenatal steroids and neonatal outcome after chorioamnionitis: a meta-analysis," BJOG, vol. 118, pp. 113-122, Jan 2011.

[78] R. W. Redline, "Inflammatory response in acute chorioamnionitis," Semin Fetal Neonatal Med, vol. 17, pp. 20-25, Feb 2012.

[79] K. E. Willet, B. W. Kramer, S. G. Kallapur, M. Ikegami, J. P. Newnham, T. J. Moss, P. D. Sly, and A. H. Jobe, "Intra-amniotic injection of IL-1 induces inflammation and maturation in fetal sheep lung," Am. J. Physiol. Lung Cell Mol. Physiol., vol. 282, pp. L411-420, Mar 2002.

[80] B. W. Kramer, T. J. Moss, K. E. Willet, J. P. Newnham, P. D. Sly, S. G. Kallapur, M. Ikegami, and A. H. Jobe, "Dose and time response after intraamniotic endotoxin in preterm lambs," Am. J. Respir. Crit. Care Med., vol. 164, pp. 982-988, Sep 2001.

[81] S. G. Kallapur, K. E. Willet, A. H. Jobe, M. Ikegami, and C. J. Bachurski, "Intra-amniotic endotoxin: chorioamnionitis precedes lung maturation in preterm lambs," Am. J. Physiol. Lung Cell Mol. Physiol., vol. 280, pp. L527536, Mar 2001.

[82] B. W. Kramer, S. Kramer, M. Ikegami, and A. H. Jobe, "Injury, inflammation, and remodeling in fetal sheep lung after intra-amniotic endotoxin," Am. J. Physiol. Lung Cell Mol. Physiol., vol. 283, pp. L452-459, Aug 2002.

[83] A. W. Gavilanes, E. Strackx, B. W. Kramer, M. Gantert, D. Van den Hove, H. Steinbusch, Y. Garnier, E. Cornips, H. Steinbusch, L. Zimmermann, and J. Vles, "Chorioamnionitis induced by intraamniotic lipopolysaccharide resulted in an interval-dependent increase in central nervous system injury in the fetal sheep," Am. J. Obstet. Gynecol., vol. 200, pp. 1-8, Apr 2009.

[84] T. G. Wolfs, W. A. Buurman, B. Zoer, R. M. Moonen, J. P. Derikx, G. Thuijls, E. Villamor, M. Gantert, Y. Garnier, L. J. Zimmermann, and B. W. Kramer, "Endotoxin induced chorioamnionitis prevents intestinal development during gestation in fetal sheep," PLoS ONE, vol. 4, p. e5837, 2009. 
[85] O. Dammann, M. J. Brinkhaus, D. B. Bartels, M. Dordelmann, F. Dressler, J. Kerk, T. Dork, and C. E. Dammann, "Immaturity, perinatal inflammation, and retinopathy of prematurity: a multi-hit hypothesis," Early Hum. Dev., vol. 85, pp. 325-329, May 2009.

[86] J. V. Been, R. F. Kornelisse, I. G. Rours, V. Lima Passos, R. R. De Krijger, and L. J. Zimmermann, "Early postnatal blood pressure in preterm infants: effects of chorioamnionitis and timing of antenatal steroids," Pediatr. Res., vol. 66, pp. 571-576, Nov 2009.

[87] A. B. Coumans, Y. Garnier, S. Supcun, A. Jensen, R. Berger, and T. H. Hasaart, "The effects of low-dose endotoxin on the umbilicoplacental circulation in preterm sheep," J. Soc. Gynecol. Investig., vol. 11, pp. 289-293, Jul 2004.

[88] K. Wouters, P. J. van Gorp, V. Bieghs, M. J. Gijbels, H. Duimel, D. Lutjohann, A. Kerksiek, R. van Kruchten, N. Maeda, B. Staels, M. van Bilsen, R. Shiri-Sverdlov, and M. H. Hofker, "Dietary cholesterol, rather than liver steatosis, leads to hepatic inflammation in hyperlipidemic mouse models of nonalcoholic steatohepatitis," Hepatology, vol. 48, pp. 474-486, Aug 2008.

[89] R. Shiri-Sverdlov, K. Wouters, P. J. van Gorp, M. J. Gijbels, B. Noel, L. Buffat, B. Staels, N. Maeda, M. van Bilsen, and M. H. Hofker, "Early dietinduced non-alcoholic steatohepatitis in APOE2 knock-in mice and its prevention by fibrates," J. Hepatol., vol. 44, pp. 732-741, Apr 2006.

[90] T. Roohey, T. N. Raju, and A. N. Moustogiannis, "Animal models for the study of perinatal hypoxic-ischemic encephalopathy: a critical analysis," Early Hum. Dev., vol. 47, pp. 115-146, Jan 1997.

[91] J. E. Rice, R. C. Vannucci, and J. B. Brierley, "The influence of immaturity on hypoxic-ischemic brain damage in the rat," Ann. Neurol., vol. 9, pp. 131141, Feb 1981.

[92] B. Bjelke, K. Andersson, S. O. Ogren, and P. Bolme, "Asphyctic lesion: proliferation of tyrosine hydroxylase-immunoreactive nerve cell bodies in the 
rat substantia nigra and functional changes in dopamine neurotransmission," Brain Res., vol. 543, pp. 1-9, Mar 1991.

[93] E. Dell'Anna, Y. Chen, E. Engidawork, K. Andersson, G. Lubec, J. Luthman, and M. Herrera-Marschitz, "Delayed neuronal death following perinatal asphyxia in rat," Exp Brain Res, vol. 115, pp. 105-115, Jun 1997.

[94] Z. Cai, F. Xiao, B. Lee, I. A. Paul, and P. G. Rhodes, "Prenatal hypoxiaischemia alters expression and activity of nitric oxide synthase in the young rat brain and causes learning deficits," Brain Res. Bull., vol. 49, pp. 359-365, Jul 1999.

[95] B. W. Kramer, M. Ikegami, and A. H. Jobe, "Intratracheal endotoxin causes systemic inflammation in ventilated preterm lambs," Am. J. Respir. Crit. Care Med., vol. 165, pp. 463-469, Feb 2002.

[96] J. P. Newnham, S. G. Kallapur, B. W. Kramer, T. J. Moss, I. Nitsos, M. Ikegami, and A. H. Jobe, "Betamethasone effects on chorioamnionitis induced by intra-amniotic endotoxin in sheep," Am. J. Obstet. Gynecol., vol. 189, pp. 1458-1466, Nov 2003.

[97] R. Berger and Y. Garnier, "Pathophysiology of perinatal brain damage," Brain Res. Brain Res. Rev., vol. 30, pp. 107-134, Aug 1999.

[98] M. Thoresen, "Hypothermia after perinatal asphyxia: selection for treatment and cooling protocol," J. Pediatr., vol. 158, pp. e45-49, Feb 2011.

[99] D. Maslinska, M. Laure-Kamionowska, A. Kaliszek, and D. Makarewicz, "Proinflammatory cytokines in injured rat brain following perinatal asphyxia," Folia Neuropathol, vol. 40, no. 4, pp. 177-182, 2002.

[100] J. M. Pradillo, D. Fernandez-Lopez, I. Garcia-Yebenes, M. Sobrado, O. Hurtado, M. A. Moro, and I. Lizasoain, "Toll-like receptor 4 is involved in neuroprotection afforded by ischemic preconditioning," J. Neurochem., vol. 109, pp. 287-294, Apr 2009. 
[101] Z. Feng, D. P. Davis, R. Sasik, H. H. Patel, J. C. Drummond, and P. M. Patel, "Pathway and gene ontology based analysis of gene expression in a rat model of cerebral ischemic tolerance," Brain Res., vol. 1177, pp. 103123, Oct 2007.

[102] R. Tyzio, R. Cossart, I. Khalilov, M. Minlebaev, C. A. Hubner, A. Represa, Y. Ben-Ari, and R. Khazipov, "Maternal oxytocin triggers a transient inhibitory switch in GABA signaling in the fetal brain during delivery," Science, vol. 314, pp. 1788-1792, Dec 2006.

[103] O. Touzani, H. Boutin, J. Chuquet, and N. Rothwell, "Potential mechanisms of interleukin-1 involvement in cerebral ischaemia," J. Neuroimmunol., vol. 100, pp. 203-215, Dec 1999.

[104] L. A. O'Neill and C. Greene, "Signal transduction pathways activated by the IL-1 receptor family: ancient signaling machinery in mammals, insects, and plants," J. Leukoc. Biol., vol. 63, pp. 650-657, Jun 1998.

[105] E. Shohami, I. Ginis, and J. M. Hallenbeck, "Dual role of tumor necrosis factor alpha in brain injury," Cytokine Growth Factor Rev., vol. 10, pp. 119130, Jun 1999.

[106] M. Roy, S. Girard, A. Larouche, H. Kadhim, and G. Sebire, "TNF-alpha system response in a rat model of very preterm brain injuries induced by lipopolysaccharide and/or hypoxia-ischemia," Am. J. Obstet. Gynecol., vol. 201, pp. 1-10, Nov 2009.

[107] H. Nawashiro, K. Tasaki, C. A. Ruetzler, and J. M. Hallenbeck, "TNF-alpha pretreatment induces protective effects against focal cerebral ischemia in mice," J. Cereb. Blood Flow Metab., vol. 17, pp. 483-490, May 1997.

[108] S. D. Lavine, F. M. Hofman, and B. V. Zlokovic, "Circulating antibody against tumor necrosis factor-alpha protects rat brain from reperfusion injury," J. Cereb. Blood Flow Metab., vol. 18, pp. 52-58, Jan 1998. 
[109] J. M. Pradillo, C. Romera, O. Hurtado, A. Cardenas, M. A. Moro, J. C. Leza, A. Davalos, J. Castillo, P. Lorenzo, and I. Lizasoain, "TNFR1 upregulation mediates tolerance after brain ischemic preconditioning," J. Cereb. Blood Flow Metab., vol. 25, pp. 193-203, Feb 2005.

[110] C. Veroni, L. Gabriele, I. Canini, L. Castiello, E. Coccia, M. E. Remoli, S. Columba-Cabezas, E. Arico, F. Aloisi, and C. Agresti, "Activation of TNF receptor 2 in microglia promotes induction of anti-inflammatory pathways," Mol. Cell. Neurosci., vol. 45, pp. 234-244, Nov 2010.

[111] M. E. Brochu, S. Girard, K. Lavoie, and G. Sebire, "Developmental regulation of the neuroinflammatory responses to LPS and/or hypoxia-ischemia between preterm and term neonates: An experimental study," J Neuroinflammation, vol. 8, p. 55, 2011.

[112] S. G. Kremlev, R. L. Roberts, and C. Palmer, "Minocycline modulates chemokine receptors but not interleukin-10 mRNA expression in hypoxicischemic neonatal rat brain," J. Neurosci. Res., vol. 85, pp. 2450-2459, Aug 2007.

[113] K. W. Park, H. G. Lee, B. K. Jin, and Y. B. Lee, "Interleukin-10 endogenously expressed in microglia prevents lipopolysaccharide-induced neurodegeneration in the rat cerebral cortex in vivo," Exp. Mol. Med., vol. 39, pp. 812-819, Dec 2007.

[114] S. Sharma, B. Yang, X. Xi, J. C. Grotta, J. Aronowski, and S. I. Savitz, "IL10 directly protects cortical neurons by activating PI-3 kinase and STAT-3 pathways," Brain Res., vol. 1373, pp. 189-194, Feb 2011.

[115] R. A. Gadient and U. H. Otten, "Interleukin-6 (IL-6)-a molecule with both beneficial and destructive potentials," Prog. Neurobiol., vol. 52, pp. 379390, Aug 1997.

[116] S. Suzuki, K. Tanaka, and N. Suzuki, "Ambivalent aspects of interleukin-6 in cerebral ischemia: inflammatory versus neurotrophic aspects," J. Cereb. Blood Flow Metab., vol. 29, pp. 464-479, Mar 2009. 
[117] S. A. Jones, S. Horiuchi, N. Topley, N. Yamamoto, and G. M. Fuller, "The soluble interleukin 6 receptor: mechanisms of production and implications in disease," FASEB J., vol. 15, pp. 43-58, Jan 2001.

[118] T. Yamashita, K. Sawamoto, S. Suzuki, N. Suzuki, K. Adachi, T. Kawase, M. Mihara, Y. Ohsugi, K. Abe, and H. Okano, "Blockade of interleukin-6 signaling aggravates ischemic cerebral damage in mice: possible involvement of Stat3 activation in the protection of neurons," J. Neurochem., vol. 94, pp. 459-468, Jul 2005.

[119] E. Yektaei-Karin, A. Moshfegh, J. Lundahl, V. Berggren, L. O. Hansson, and G. Marchini, "The stress of birth enhances in vitro spontaneous and IL8-induced neutrophil chemotaxis in the human newborn," Pediatr Allergy Immunol, vol. 18, pp. 643-651, Dec 2007.

[120] A. Munck, P. M. Guyre, and N. J. Holbrook, "Physiological functions of glucocorticoids in stress and their relation to pharmacological actions," Endocr. Rev., vol. 5, no. 1, pp. 25-44, 1984.

[121] L. Wang, W. Zhang, and Y. Zhao, "The study of maternal and fetal plasma catecholamines levels during pregnancy and delivery," $J$ Perinat Med, vol. 27, no. 3, pp. 195-198, 1999.

[122] A. M. Gulmezoglu, K. Mahomed, G. J. Hofmeyr, V. C. Nikodem, and T. Kramer, "Fetal and maternal catecholamine levels at delivery," J Perinat Med, vol. 24, no. 6, pp. 687-691, 1996.

[123] D. Martin, N. Chinookoswong, and G. Miller, "The interleukin-1 receptor antagonist (rhIL-1ra) protects against cerebral infarction in a rat model of hypoxia-ischemia," Exp. Neurol., vol. 130, pp. 362-367, Dec 1994.

[124] Y. Sun, J. W. Calvert, and J. H. Zhang, "Neonatal hypoxia/ischemia is associated with decreased inflammatory mediators after erythropoietin administration," Stroke, vol. 36, pp. 1672-1678, Aug 2005. 
[125] C. H. Nijboer, C. J. Heijnen, F. Groenendaal, M. J. May, F. van Bel, and A. Kavelaars, "Strong neuroprotection by inhibition of NF-kappaB after neonatal hypoxia-ischemia involves apoptotic mechanisms but is independent of cytokines," Stroke, vol. 39, pp. 2129-2137, Jul 2008.

[126] J. R. Caso, J. M. Pradillo, O. Hurtado, P. Lorenzo, M. A. Moro, and I. Lizasoain, "Toll-like receptor 4 is involved in brain damage and inflammation after experimental stroke," Circulation, vol. 115, pp. 1599-1608, Mar 2007.

[127] J. E. Lawn, K. Kerber, C. Enweronu-Laryea, and S. Cousens, "3.6 million neonatal deaths-what is progressing and what is not?," Semin. Perinatol., vol. 34, pp. 371-386, Dec 2010.

[128] U. Hogberg, "The World Health Report 2005: "make every mother and child count" - including Africans," Scand J Public Health, vol. 33, no. 6, pp. 409$411,2005$.

[129] M. Matuszczak-Wleklak and M. Szymankiewicz, "[Perinatal asphyxia, known topic, new diagnostic prospects-advisability of troponin $\mathrm{T}$ in perinatal medicine]," Ginekol. Pol., vol. 74, pp. 487-493, Jun 2003.

[130] N. Ueda, G. P. Kaushal, X. Hong, and S. V. Shah, "Role of enhanced ceramide generation in DNA damage and cell death in chemical hypoxic injury to LLC-PK1 cells," Kidney Int., vol. 54, pp. 399-406, Aug 1998.

[131] F. F. Bitar, S. Mroueh, M. El Khatib, H. Bitar, M. Tarrabain, M. El Sabban, M. Obeid, M. Nasser, and G. S. Dbaibo, "Tissue-specific ceramide response in the chronically hypoxic rat model mimicking cyanotic heart disease," Prostaglandins Other Lipid Mediat., vol. 72, pp. 155-163, Nov 2003.

[132] R. Ohtani, H. Tomimoto, T. Kondo, H. Wakita, I. Akiguchi, H. Shibasaki, and T. Okazaki, "Upregulation of ceramide and its regulating mechanism in a rat model of chronic cerebral ischemia," Brain Res., vol. 1023, pp. 31-40, Oct 2004. 
[133] K. Hanada, K. Kumagai, S. Yasuda, Y. Miura, M. Kawano, M. Fukasawa, and M. Nishijima, "Molecular machinery for non-vesicular trafficking of ceramide," Nature, vol. 426, pp. 803-809, Dec 2003.

[134] S. S. Xu, C. L. Yan, L. M. Liu, and Q. L. Zeng, "[Effects of different cell lysis buffers on protein quantification]," Zhejiang Da Хие Хие Вао Yi Хие Ban, vol. 37, pp. 45-50, Jan 2008.

[135] J. E. Groener, B. J. Poorthuis, S. Kuiper, M. T. Helmond, C. E. Hollak, and J. M. Aerts, "HPLC for simultaneous quantification of total ceramide, glucosylceramide, and ceramide trihexoside concentrations in plasma," Clin. Chem., vol. 53, pp. 742-747, Apr 2007.

[136] R. J. Perry and N. D. Ridgway, "Molecular mechanisms and regulation of ceramide transport," Biochim. Biophys. Acta, vol. 1734, pp. 220-234, Jun 2005.

[137] Y. Chen, I. Ginis, and J. M. Hallenbeck, "The protective effect of ceramide in immature rat brain hypoxia-ischemia involves up-regulation of bcl-2 and reduction of TUNEL-positive cells," J. Cereb. Blood Flow Metab., vol. 21, pp. 34-40, Jan 2001.

[138] Y. A. Hannun, L. M. Obeid, and R. A. Wolff, "The novel second messenger ceramide: identification, mechanism of action, and cellular activity," $A d v$. Lipid Res., vol. 25, pp. 43-64, 1993.

[139] J. Zhang, N. Alter, J. C. Reed, C. Borner, L. M. Obeid, and Y. A. Hannun, "Bcl-2 interrupts the ceramide-mediated pathway of cell death," Proc. Natl. Acad. Sci. U.S.A., vol. 93, pp. 5325-5328, May 1996.

[140] D. M. Hockenbery, Z. N. Oltvai, X. M. Yin, C. L. Milliman, and S. J. Korsmeyer, "Bcl-2 functions in an antioxidant pathway to prevent apoptosis," Cell, vol. 75, pp. 241-251, Oct 1993.

[141] I. Ginis, U. Schweizer, M. Brenner, J. Liu, N. Azzam, M. Spatz, and J. M. Hallenbeck, "TNF-alpha pretreatment prevents subsequent activation of cul- 
tured brain cells with TNF-alpha and hypoxia via ceramide," Am. J. Physiol., vol. 276, pp. C1171-1183, May 1999.

[142] J. E. Lawn, S. Cousens, Z. A. Bhutta, G. L. Darmstadt, J. Martines, V. Paul, R. Knippenberg, H. Fogstadt, P. Shetty, and R. Horton, "Why are 4 million newborn babies dying each year?," Lancet, vol. 364, no. 9432, pp. 399-401, 2004.

[143] G. S. Dbaibo, L. M. Obeid, and Y. A. Hannun, "Tumor necrosis factor-alpha (TNF-alpha) signal transduction through ceramide. Dissociation of growth inhibitory effects of TNF-alpha from activation of nuclear factor-kappa B," J. Biol. Chem., vol. 268, pp. 17762-17766, Aug 1993.

[144] E. Strackx, D. L. Van den Hove, H. P. Steinbusch, H. W. Steinbusch, J. S. Vles, C. E. Blanco, and A. W. Gavilanes, "A combined behavioral and morphological study on the effects of fetal asphyxia on the nigrostriatal dopaminergic system in adult rats," Exp. Neurol., vol. 211, pp. 413-422, Jun 2008.

[145] B. F. El-Khodor and P. Boksa, "Differential vulnerability of male versus female rats to long-term effects of birth insult on brain catecholamine levels," Exp. Neurol., vol. 182, pp. 208-219, Jul 2003.

[146] Y. Q. Zhang, J. Shi, G. Rajakumar, A. L. Day, and J. W. Simpkins, "Effects of gender and estradiol treatment on focal brain ischemia," Brain Res., vol. 784, pp. 321-324, Feb 1998.

[147] J. Stiban, R. Tidhar, and A. H. Futerman, "Ceramide synthases: roles in cell physiology and signaling," Adv. Exp. Med. Biol., vol. 688, pp. 60-71, 2010.

[148] J. Jin, Q. Hou, T. D. Mullen, Y. H. Zeidan, J. Bielawski, J. M. Kraveka, A. Bielawska, L. M. Obeid, Y. A. Hannun, and Y. T. Hsu, "Ceramide generated by sphingomyelin hydrolysis and the salvage pathway is involved in hypoxia/reoxygenation-induced Bax redistribution to mitochondria in NT-2 cells," J. Biol. Chem., vol. 283, pp. 26509-26517, Sep 2008. 
[149] S. Pyne, S. C. Lee, J. Long, and N. J. Pyne, "Role of sphingosine kinases and lipid phosphate phosphatases in regulating spatial sphingosine 1-phosphate signalling in health and disease," Cell. Signal., vol. 21, pp. 14-21, Jan 2009.

[150] T. Hla and V. Brinkmann, "Sphingosine 1-phosphate (S1P): Physiology and the effects of S1P receptor modulation," Neurology, vol. 76, pp. 3-8, Feb 2011.

[151] G. M. Strub, M. Maceyka, N. C. Hait, S. Milstien, and S. Spiegel, "Extracellular and intracellular actions of sphingosine-1-phosphate," Adv. Exp. Med. Biol., vol. 688, pp. 141-155, 2010.

[152] M. Rapanelli, L. R. Frick, and B. S. Zanutto, "Learning an operant conditioning task differentially induces gliogenesis in the medial prefrontal cortex and neurogenesis in the hippocampus," PLoS ONE, vol. 6, no. 2, p. e14713, 2011.

[153] N. J. Haughey, V. V. Bandaru, M. Bae, and M. P. Mattson, "Roles for dysfunctional sphingolipid metabolism in Alzheimer's disease neuropathogenesis," Biochim. Biophys. Acta, vol. 1801, pp. 878-886, Aug 2010.

[154] A. Venerosi, D. Cutuli, F. Chiarotti, and G. Calamandrei, "C-section birth per se or followed by acute global asphyxia altered emotional behaviour in neonate and adult rats," Behav. Brain Res., vol. 168, pp. 56-63, Mar 2006.

[155] R. Weitzdoerfer, N. Gerstl, D. Pollak, H. Hoeger, W. Dreher, and G. Lubec, "Long-term influence of perinatal asphyxia on the social behavior in aging rats," Gerontology, vol. 50, no. 4, pp. 200-205, 2004.

[156] S. K. Shankar, "Biology of aging brain," Indian J Pathol Microbiol, vol. 53, no. 4, pp. 595-604, 2010.

[157] D. E. Smith, P. R. Rapp, H. M. McKay, J. A. Roberts, and M. H. Tuszynski, "Memory impairment in aged primates is associated with focal death of cortical neurons and atrophy of subcortical neurons," J. Neurosci., vol. 24, pp. 4373-4381, May 2004. 
[158] F. Bernal, J. Saura, J. Ojuel, and N. Mahy, "Differential vulnerability of hippocampus, basal ganglia, and prefrontal cortex to long-term NMDA excitotoxicity," Exp. Neurol., vol. 161, pp. 686-695, Feb 2000.

[159] M. Alonso-Spilsbury, D. Mota-Rojas, D. Villanueva-Garcia, J. MartinezBurnes, H. Orozco, R. Ramirez-Necoechea, A. L. Mayagoitia, and M. E. Trujillo, "Perinatal asphyxia pathophysiology in pig and human: a review," Anim. Reprod. Sci., vol. 90, pp. 1-30, Nov 2005.

[160] G. D. Hankins, S. Koen, A. F. Gei, S. M. Lopez, J. W. Van Hook, and G. D. Anderson, "Neonatal organ system injury in acute birth asphyxia sufficient to result in neonatal encephalopathy," Obstet Gynecol, vol. 99, pp. 688-691, May 2002.

[161] P. Shah, S. Riphagen, J. Beyene, and M. Perlman, "Multiorgan dysfunction in infants with post-asphyxial hypoxic-ischaemic encephalopathy," Arch. Dis. Child. Fetal Neonatal Ed., vol. 89, pp. F152-155, Mar 2004.

[162] M. Karlsson, M. Blennow, A. Nemeth, and B. Winbladh, "Dynamics of hepatic enzyme activity following birth asphyxia," Acta Paediatr., vol. 95, pp. 1405-1411, Nov 2006.

[163] M. T. Islam, M. N. Islam, A. H. Mollah, M. A. Hoque, M. A. Hossain, F. Nazir, and M. M. Ahsan, "Status of liver enzymes in babies with perinatal asphyxia," Mymensingh Med J, vol. 20, pp. 446-449, Jul 2011.

[164] M. Mari and J. C. Fernandez-Checa, "Sphingolipid signalling and liver diseases," Liver Int., vol. 27, pp. 440-450, May 2007.

[165] C. F. Rueda-Clausen, V. W. Dolinsky, J. S. Morton, S. D. Proctor, J. R. Dyck, and S. T. Davidge, "Hypoxia-induced intrauterine growth restriction increases the susceptibility of rats to high-fat diet-induced metabolic syndrome," Diabetes, vol. 60, pp. 507-516, Feb 2011. 
[166] W. X. Ding and X. M. Yin, "Dissection of the multiple mechanisms of TNFalpha-induced apoptosis in liver injury," J. Cell. Mol. Med., vol. 8, no. 4, pp. 445-454, 2004.

[167] S. T. Zhai, G. Y. Liu, F. Xue, G. P. Sun, L. Liang, W. Chen, G. D. Xu, J. J. Li, J. Yang, and T. B. Liang, "Changes of sphingolipids profiles after ischemiareperfusion injury in the rat liver," Chin. Med. J., vol. 122, pp. 3025-3031, Dec 2009.

[168] L. Llacuna, M. Mari, C. Garcia-Ruiz, J. C. Fernandez-Checa, and A. Morales, "Critical role of acidic sphingomyelinase in murine hepatic ischemia-reperfusion injury," Hepatology, vol. 44, pp. 561-572, Sep 2006.

[169] V. Bieghs, E. Vlassaks, A. Custers, P. J. van Gorp, M. J. Gijbels, A. Bast, O. Bekers, L. J. Zimmermann, D. Lutjohann, J. W. Voncken, A. W. Gavilanes, B. W. Kramer, and R. Shiri-Sverdlov, "Chorioamnionitis induced hepatic inflammation and disturbed lipid metabolism in fetal sheep," Pediatr. Res., vol. 68, pp. 466-472, Dec 2010.

[170] G. Li Volti, T. Musumeci, R. Pignatello, P. Murabito, I. Barbagallo, C. Carbone, A. Gullo, and G. Puglisi, "Antioxidant potential of different melatoninloaded nanomedicines in an experimental model of sepsis," Exp. Biol. Med. (Maywood), vol. 237, pp. 670-677, Jun 2012.

[171] V. T. Maddaiah, "Glutathione correlates with lipid peroxidation in liver mitochondria of triiodothyronine-injected hypophysectomized rats," FASEB J., vol. 4, pp. 1513-1518, Mar 1990.

[172] H. A. Al-Aubaidy and H. F. Jelinek, "8-Hydroxy-2-deoxy-guanosine identifies oxidative DNA damage in a rural prediabetes cohort," Redox Rep., vol. 15 , no. 4, pp. 155-160, 2010.

[173] G. Marrazzo, P. Bosco, F. La Delia, G. Scapagnini, C. Di Giacomo, M. Malaguarnera, F. Galvano, A. Nicolosi, and G. Li Volti, "Neuroprotective effect of silibinin in diabetic mice," Neurosci. Lett., vol. 504, pp. 252-256, Oct 2011. 
[174] A. V. Alessenko, E. I. Galperin, L. B. Dudnik, V. G. Korobko, E. S. Mochalova, L. V. Platonova, L. N. Shingarova, N. I. Shono, and M. A. Shupik, "Role of tumor necrosis factor alpha and sphingomyelin cycle activation in the induction of apoptosis by ischemia/reperfusion of the liver," Biochemistry Mosc., vol. 67, pp. 1347-1355, Dec 2002.

[175] L. A. Bemmel, W. W. Hack, C. A. Seldenrijk, and C. M. Kneepkens, "Extensive hepatic necrosis in a premature infant," J. Pediatr. Gastroenterol. Nutr., vol. 14, pp. 228-231, Feb 1992.

[176] O. Kilavuz and K. Vetter, "Is the liver of the fetus the 4th preferential organ for arterial blood supply besides brain, heart, and adrenal glands?," J Perinat Med, vol. 27, no. 2, pp. 103-106, 1999.

[177] J. J. Lemasters, C. J. Stemkowski, S. Ji, and R. G. Thurman, "Cell surface changes and enzyme release during hypoxia and reoxygenation in the isolated, perfused rat liver," J. Cell Biol., vol. 97, pp. 778-786, Sep 1983.

[178] N. Maulik, R. M. Engelman, J. A. Rousou, J. E. Flack, D. Deaton, and D. K. Das, "Ischemic preconditioning reduces apoptosis by upregulating anti-death gene Bcl-2," Circulation, vol. 100, pp. I369-375, Nov 1999.

[179] A. S. Parsadanian, Y. Cheng, C. R. Keller-Peck, D. M. Holtzman, and W. D. Snider, "Bcl-xL is an antiapoptotic regulator for postnatal CNS neurons," $J$. Neurosci., vol. 18, pp. 1009-1019, Feb 1998.

[180] C. Wiessner, P. R. Allegrini, K. Rupalla, D. Sauer, T. Oltersdorf, A. L. McGregor, S. Bischoff, B. W. Bottiger, and H. van der Putten, "Neuron-specific transgene expression of Bcl-XL but not Bcl-2 genes reduced lesion size after permanent middle cerebral artery occlusion in mice," Neurosci. Lett., vol. 268, pp. 119-122, Jun 1999.

[181] E. Bibby and A. Stewart, "The epidemiology of preterm birth," Neuro Endocrinol. Lett., vol. 25 Suppl 1, pp. 43-47, Dec 2004. 
[182] W. Khovidhunkit, M. S. Kim, R. A. Memon, J. K. Shigenaga, A. H. Moser, K. R. Feingold, and C. Grunfeld, "Effects of infection and inflammation on lipid and lipoprotein metabolism: mechanisms and consequences to the host," J. Lipid Res., vol. 45, pp. 1169-1196, Jul 2004.

[183] M. Baron, "Induction of embryonic hematopoietic and endothelial stem/progenitor cells by hedgehog-mediated signals," Differentiation, vol. 68, pp. 175-185, Oct 2001.

[184] S. J. Klebanoff, "Myeloperoxidase: friend and foe," J. Leukoc. Biol., vol. 77, pp. 598-625, May 2005.

[185] M. A. Fischer, T. J. Gransier, L. M. Beckers, O. Bekers, A. Bast, and G. R. Haenen, "Determination of the antioxidant capacity in blood," Clin. Chem. Lab. Med., vol. 43, pp. 735-740, 2005.

[186] K. Tsunoda, S. Hamato, S. Kurosawa, A. Shirato, Y. Kurosawa, K. Fujimaki, M. Muto, and K. Sato, "Apolipoprotein C polymorphism in sheep: allele frequencies and association with plasma lipid and lipoprotein levels," Biochem. Genet., vol. 40, pp. 27-39, Feb 2002.

[187] F. Uyanik, "The effects of dietary chromium supplementation on some blood parameters in sheep," Biol Trace Elem Res, vol. 84, pp. 93-101, 2001.

[188] W. T. Friedewald, R. I. Levy, and D. S. Fredrickson, "Estimation of the concentration of low-density lipoprotein cholesterol in plasma, without use of the preparative ultracentrifuge," Clin. Chem., vol. 18, pp. 499-502, Jun 1972.

[189] M. C. Neville, "Adaptation of maternal lipid flux to pregnancy: research needs," Eur J Clin Nutr, vol. 53 Suppl 1, pp. S120-123, Apr 1999.

[190] H. Tilg and G. S. Hotamisligil, "Nonalcoholic fatty liver disease: Cytokineadipokine interplay and regulation of insulin resistance," Gastroenterology, vol. 131, pp. 934-945, Sep 2006. 
[191] A. W. Gavilanes, M. Gantert, E. Strackx, L. J. Zimmermann, S. Seeldrayers, J. S. Vles, and B. W. Kramer, "Increased EEG delta frequency corresponds to chorioamnionitis-related brain injury," Front Biosci (Schol Ed), vol. 2, pp. 432-438, 2010.

[192] I. R. Sosenko and A. H. Jobe, "Intraamniotic endotoxin increases lung antioxidant enzyme activity in preterm lambs," Pediatr. Res., vol. 53, pp. 679683, Apr 2003.

[193] A. H. Jobe, J. P. Newnham, K. E. Willet, T. J. Moss, M. Gore Ervin, J. F. Padbury, P. Sly, and M. Ikegami, "Endotoxin-induced lung maturation in preterm lambs is not mediated by cortisol," Am. J. Respir. Crit. Care Med., vol. 162, pp. 1656-1661, Nov 2000.

[194] F. C. Cheah, A. H. Jobe, T. J. Moss, J. P. Newnham, and S. G. Kallapur, "Oxidative stress in fetal lambs exposed to intra-amniotic endotoxin in a chorioamnionitis model," Pediatr. Res., vol. 63, pp. 274-279, Mar 2008.

[195] V. Caille, P. Bossi, D. Grimaldi, and A. Vieillard-Baro, "[physiopathology of severe sepsis]," Presse Med, vol. 33, pp. 256-261, Feb 2004.

[196] F. Tacke, T. Luedde, and C. Trautwein, "Inflammatory pathways in liver homeostasis and liver injury," Clin Rev Allergy Immunol, vol. 36, pp. 4-12, Feb 2009.

[197] B. Mundt, T. Wirth, L. Zender, M. Waltemathe, C. Trautwein, M. P. Manns, F. Kuhnel, and S. Kubicka, "Tumour necrosis factor related apoptosis inducing ligand (TRAIL) induces hepatic steatosis in viral hepatitis and after alcohol intake," Gut, vol. 54, pp. 1590-1596, Nov 2005.

[198] S. Chikano, K. Sawada, T. Shimoyama, S. I. Kashiwamura, A. Sugihara, K. Sekikawa, N. Terada, K. Nakanishi, and H. Okamura, "IL-18 and IL12 induce intestinal inflammation and fatty liver in mice in an IFN-gamma dependent manner," Gut, vol. 47, pp. 779-786, Dec 2000. 
[199] C. Villar-Palasi and J. Larner, "Glycogen metabolism and glycolytic enzymes," Annu. Rev. Biochem., vol. 39, pp. 639-672, 1970.

[200] V. Loria, I. Dato, F. Graziani, and L. M. Biasucci, "Myeloperoxidase: a new biomarker of inflammation in ischemic heart disease and acute coronary syndromes," Mediators Inflamm., vol. 2008, p. 135625, 2008.

[201] S. S. Rensen, Y. Slaats, J. Nijhuis, A. Jans, V. Bieghs, A. Driessen, E. Malle, J. W. Greve, and W. A. Buurman, "Increased hepatic myeloperoxidase activity in obese subjects with nonalcoholic steatohepatitis," Am. J. Pathol., vol. 175, pp. 1473-1482, Oct 2009.

[202] O. D. Saugstad, "Oxidative stress in the newborn-a 30-year perspective," Biol. Neonate, vol. 88, pp. 228-236, 2005.

[203] E. F. Sato, E. Nakagawa, K. Hiramoto, S. Yamamasu, I. MoriyamaShimamoto, and M. Inoue, "Oxidative stress promotes the regression of fetal liver hemopoiesis,” Biochemistry Mosc., vol. 69, pp. 18-22, Jan 2004.

[204] L. Pari and A. Prasath, "Efficacy of caffeic acid in preventing nickel induced oxidative damage in liver of rats," Chem. Biol. Interact., vol. 173, pp. 77-83, May 2008.

[205] P. P. Ruvolo, "Intracellular signal transduction pathways activated by ceramide and its metabolites," Pharmacol. Res., vol. 47, pp. 383-392, May 2003.

[206] J. D. Browning and J. D. Horton, "Molecular mediators of hepatic steatosis and liver injury," J. Clin. Invest., vol. 114, pp. 147-152, Jul 2004.

[207] J. V. Been and L. J. Zimmermann, "Histological chorioamnionitis and respiratory outcome in preterm infants," Arch. Dis. Child. Fetal Neonatal Ed., vol. 94, pp. F218-225, May 2009.

[208] T. J. Roseboom, J. H. van der Meulen, A. C. Ravelli, C. Osmond, D. J. Barker, and O. P. Bleker, "Effects of prenatal exposure to the Dutch famine 
on adult disease in later life: an overview," Mol. Cell. Endocrinol., vol. 185, pp. 93-98, Dec 2001.

[209] A. J. Lee, V. A. Lambermont, J. J. Pillow, G. R. Polglase, I. Nitsos, J. P. Newnham, M. W. Beilharz, S. G. Kallapur, A. H. Jobe, and B. W. Kramer, "Fetal responses to lipopolysaccharide-induced chorioamnionitis alter immune and airway responses in 7-week-old sheep," Am. J. Obstet. Gynecol., vol. 204, pp. 17-24, Apr 2011.

[210] R. L. Shaner, J. C. Allegood, H. Park, E. Wang, S. Kelly, C. A. Haynes, M. C. Sullards, and A. H. Merrill, "Quantitative analysis of sphingolipids for lipidomics using triple quadrupole and quadrupole linear ion trap mass spectrometers," J. Lipid Res., vol. 50, pp. 1692-1707, Aug 2009.

[211] N. Alkhouri, L. J. Dixon, and A. E. Feldstein, "Lipotoxicity in nonalcoholic fatty liver disease: not all lipids are created equal," Expert Rev Gastroenterol Hepatol, vol. 3, pp. 445-451, Aug 2009.

[212] G. Yang, L. Badeanlou, J. Bielawski, A. J. Roberts, Y. A. Hannun, and F. Samad, "Central role of ceramide biosynthesis in body weight regulation, energy metabolism, and the metabolic syndrome," Am. J. Physiol. Endocrinol. Metab., vol. 297, pp. E211-224, Jul 2009.

[213] M. Pejznochova, M. Tesarova, H. Hansikova, M. Magner, T. Honzik, K. Vinsova, Z. Hajkova, V. Havlickova, and J. Zeman, "Mitochondrial DNA content and expression of genes involved in mtDNA transcription, regulation and maintenance during human fetal development," Mitochondrion, vol. 10, pp. 321-329, Jun 2010.

[214] Y. Wei, S. E. Clark, J. P. Thyfault, G. M. Uptergrove, W. Li, A. T. WhaleyConnell, C. M. Ferrario, J. R. Sowers, and J. A. Ibdah, "Oxidative stressmediated mitochondrial dysfunction contributes to angiotensin II-induced nonalcoholic fatty liver disease in transgenic Ren2 rats," Am. J. Pathol., vol. 174, pp. 1329-1337, Apr 2009. 
[215] A. H. Jobe and M. Ikegami, "Antenatal infection/inflammation and postnatal lung maturation and injury," Respir. Res., vol. 2, no. 1, pp. 27-32, 2001.

[216] E. Normann, T. Lacaze-Masmonteil, F. Eaton, L. Schwendimann, P. Gressens, and B. Thebaud, "A novel mouse model of Ureaplasma-induced perinatal inflammation: effects on lung and brain injury," Pediatr. Res., vol. 65, pp. 430-436, Apr 2009.

[217] B. A. Yoder, J. J. Coalson, V. T. Winter, T. Siler-Khodr, L. B. Duffy, and G. H. Cassell, "Effects of antenatal colonization with ureaplasma urealyticum on pulmonary disease in the immature baboon," Pediatr. Res., vol. 54, pp. 797-807, Dec 2003.

[218] K. M. Godfrey, P. D. Gluckman, and M. A. Hanson, "Developmental origins of metabolic disease: life course and intergenerational perspectives," Trends Endocrinol. Metab., vol. 21, pp. 199-205, Apr 2010.

[219] S. J. Schwarzenberg, C. J. Potter, and S. A. Berry, "Developmental regulation of the hepatic acute phase response," Am. J. Physiol., vol. 261, pp. C461-466, Sep 1991.

[220] M. Patricolo, A. Zangari, N. Paolocci, F. Magni, M. P. Viola-Magni, L. A. Hernandez-Mena, L. Capuano, and M. Rivosecchi, "In utero partial liver resection in the rabbit model: a study on fetal tissue regeneration," Fetal. Diagn. Ther., vol. 12, no. 4, pp. 232-235, 1997.

[221] J. A. Duffield, T. Vuocolo, R. Tellam, B. S. Yuen, B. S. Muhlhausler, and I. C. McMillen, "Placental restriction of fetal growth decreases IGF1 and leptin mRNA expression in the perirenal adipose tissue of late gestation fetal sheep," Am. J. Physiol. Regul. Integr. Comp. Physiol., vol. 294, pp. R14131419, May 2008.

[222] C. Demeilliers, C. Maisonneuve, A. Grodet, A. Mansouri, R. Nguyen, M. Tinel, P. Letteron, C. Degott, G. Feldmann, D. Pessayre, and B. Fromenty, "Impaired adaptive resynthesis and prolonged depletion of hepatic 
mitochondrial DNA after repeated alcohol binges in mice," Gastroenterology, vol. 123, pp. 1278-1290, Oct 2002.

[223] R. A. Memon, W. M. Holleran, A. H. Moser, T. Seki, Y. Uchida, J. Fuller, J. K. Shigenaga, C. Grunfeld, and K. R. Feingold, "Endotoxin and cytokines increase hepatic sphingolipid biosynthesis and produce lipoproteins enriched in ceramides and sphingomyelin," Arterioscler. Thromb. Vasc. Biol., vol. 18, pp. 1257-1265, Aug 1998.

[224] C. Zimmermann, I. Ginis, K. Furuya, D. Klimanis, C. Ruetzler, M. Spatz, and J. M. Hallenbeck, "Lipopolysaccharide-induced ischemic tolerance is associated with increased levels of ceramide in brain and in plasma," Brain Res., vol. 895, pp. 59-65, Mar 2001.

[225] R. Medzhitov, "Origin and physiological roles of inflammation," Nature, vol. 454, pp. 428-435, Jul 2008.

[226] I. Burd, A. Brown, J. M. Gonzalez, J. Chai, and M. A. Elovitz, "A mouse model of term chorioamnionitis: unraveling causes of adverse neurological outcomes," Reprod Sci, vol. 18, pp. 900-907, Sep 2011.

[227] B. J. Stoll, N. I. Hansen, I. Adams-Chapman, A. A. Fanaroff, S. R. Hintz, B. Vohr, and R. D. Higgins, "Neurodevelopmental and growth impairment among extremely low-birth-weight infants with neonatal infection," JAMA, vol. 292, pp. 2357-2365, Nov 2004.

[228] R. C. Bone, "Immunologic dissonance: a continuing evolution in our understanding of the systemic inflammatory response syndrome (SIRS) and the multiple organ dysfunction syndrome (MODS)," Ann. Intern. Med., vol. 125, pp. 680-687, Oct 1996.

[229] L. Cornette, "Fetal and neonatal inflammatory response and adverse outcome," Semin Fetal Neonatal Med, vol. 9, pp. 459-470, Dec 2004.

[230] V. S. Ten and A. Starkov, "Hypoxic-ischemic injury in the developing brain: 
the role of reactive oxygen species originating in mitochondria," Neurol Res Int, vol. 2012, p. 542976, 2012.

[231] M. Adlinolfi, "Infectious diseases in pregnancy, cytokines and neurological impairment: an hypothesis," Dev Med Child Neurol, vol. 35, pp. 549-553, Jun 1993.

[232] A. Leviton, "Preterm birth and cerebral palsy: is tumor necrosis factor the missing link?," Dev Med Child Neurol, vol. 35, pp. 553-558, Jun 1993.

[233] R. Gomez, R. Romero, F. Ghezzi, B. H. Yoon, M. Mazor, and S. M. Berry, "The fetal inflammatory response syndrome," Am. J. Obstet. Gynecol., vol. 179, pp. 194-202, Jul 1998.

[234] B. W. Kramer, "Chorioamnionitis - new ideas from experimental models," Neonatology, vol. 99, no. 4, pp. 320-325, 2011. 

Inflammatie is een belangrijke component in normale ontwikkeling. Echter, infectie en inflammatie kunnen ook leiden tot schade in verschillende organen. Perinatale asfyxie en chorioamnionitis zijn twee belangrijke neonatale complicaties die wereldwijd leiden tot ernstige orgaan afwijkingen en zelfs neonatale sterfte. Inflammatie zou in beide complicaties een belangrijke factor zijn. Bijgevolg hebben we in dit proefschrift gekeken naar onderliggende mechanismen van inflammatie van deze twee geboorte complicaties om zo tot nieuwe inzichten te komen en targets voor therapieën te achterhalen.

In hoofdstuk 2 hadden we de hypothese dat de inflammatoire respons, die veroorzaakt wordt door perinatale asfyxie, kan verminderd worden door foetale asfyctische preconditionering toe te passen. We hebben aangetoond dat na foetale asfyxie minder IL- $1 \beta$, TNF- $\alpha$ en IL-10 worden geproduceerd. Wanneer dit foetaal insult vervolgens gecombineerd wordt met een perinataal asfyctisch insult, zijn de cytokine levels vergelijkbaar met de controle dieren. Deze bevindingen geven aan dat inflammatie zeker een belangrijke component is in de schade die veroorzaakt wordt door perinatale asfyxie. Echter, het verminderen van de inflammatoire cytokine respons door bijvoorbeeld foetale asfyctische preconditionering toe te passen heeft een beschermend effect op de pasgeborene.

In hoofdstuk 3 hebben we in meer detail gekeken naar het effect van de inflammatoire respons door te kijken naar ceramide levels. Het is namelijk geweten dat ceramides een link kunnen voorzien tussen de productie van inflammatoire cytokines en de uiteindelijke celdood. Een verband tussen de productie van cytokines, ceramides en het ontstaan van celdood (apoptose) werd inderdaad geobserveerd na foetale asfyxie. In hoeverre deze veranderingen uiteindelijk zorgen voor de geobserveerde neuroprotectie is nog niet duidelijk en moet verder onderzocht worden.

In hoofdstuk 4 werd de hypothese gesteld dat de inflammatoire effecten ook nog zichtbaar zijn acht maanden na geboorte angezien perinatale asfyxie langdurige problemen veroorzaakt in spontane motoractiviteit, leren en geheugen. Alhoewel 
geen verschillen geobserveerd konden worden in cytokine levels, waren er nog steeds verschillen zichtbaar in genen belangrijk in het ceramide metabolisme. Ook konden we nog steeds een neuroprotectief effect van foetale asfyctische preconditionering aantonen in de prefrontale cortex.

In hoofdstuk 5 werd de impact van foetale en perinatale asfyxie op de lever bestudeerd. Net zoals in het brein vonden we een associatie tussen de productie van cytokines en ceramides. Een interessante observatie was dat asfyctische preconditionering ook een beschermend effect op de lever lijkt te hebben door het beïnvloeden van de inflammatoire, apoptotische en oxidatieve respons. Ondanks de acute veranderingen in inflammatie en apoptose, waren er geen significante indicaties voor leverschade. Op lange termijn leek perinatale asfyxie geen dramatische impact te hebben op de normale lever pathologie.

In hoofdstuk 6 hebben we de impact van chorioamnionitis op de lever bestudeerd. De hypothese was dat chorioamnionitis kan zorgen voor een ontstekingsreactie in de foetale lever, leidend tot verstoringen in het lipide metabolisme. Met behulp van een schapen model, hebben we aangetoond dat chorioamnionitis zorgt voor een verhoogde ontstekingsreactie met leverschade als gevolg. Bovendien waren lipide en glucose metabolisme verstoord in deze dieren. Daarom kunnen we besluiten dat in de pathogenese van chorioamnionitis de lever een belangrijke rol speelt.

In hoofdstuk 7 zijn we vervolgens gaan kijken of chorioamnionitis ook gedurende het postnatale leven nog geassocieerd is met ontsteking in de lever en verstoord lipide metabolisme. Op een leeftijd van zeven weken hebben we aangetoond dat triglyceriden in de lever en cholesterol in het plasma verhoogd waren in die dieren die blootgesteld waren aan chorioamnionitis. Deze veranderingen in lipide levels waren geassocieerd met sphingolipide veranderingen. Bijgevolg kunnen we besluiten dat dieren die blootgesteld zijn aan chorioamnionitis meer vatbaar zijn voor het ontwikkelen van leveronsteking en metabole ziekten tijdens het volwassen leven. 


\section{List of abbreviations}

8-OH-dG 8-hydroxy-2-deoxy Guanosine

ALT Alanine transaminase

ALP Alkaline phosphatase

AST Aspartate aminotranferase

aSMase Acid sphingomyelinase

ATP Adenosine triphosphate

BSA Bovine serum albumin

C Control

C-section Caesarean section

CAO Carotid artery occlusion

CERT Ceramide transporter

CNS Central nervous system

CSF Cerebrospinal fluid

CPU Caudate-putamen

DAPI 4', 6'-diamidino-2'-phenylindole

E Embryonic day

EDTA Ethylendiamine-tetra-acetic acid

EEG Electroencephalogram

ER Endoplasmatic reticulum

FA Fetal ashyxia

FCS Fetal calf serum

FFA Free fatty acid

FIRS Fetal inflammatory response syndrome

GA Gestational age

GalCer Galactosylceramide

GlcCer Glycosylceramide

GAPDH Glyceraldehyde-3-phosphate dehydrogenase

GFAP Glial fibrillary acidic protein

GGT Gamma glutamyl transferase

GPBP Goodpasture antigen-binding protein 


$\begin{array}{ll}\text { GSH } & \text { Glutathione } \\ \text { GST } & \text { Glutathione S-transferase } \\ \text { HDL } & \text { High density lipoprotein } \\ \text { H/E } & \text { Haematoxylin/Eosin } \\ \text { HIE } & \text { Hypoxic-ischemic encephalopathy } \\ \text { HPRT } & \text { Hypoxanthin-guanin-phosphoribosyl-transferase } \\ \text { IL } & \text { Interleukin } \\ \text { IFN } & \text { Interferon } \\ \text { Ig } & \text { Immunoglobulin } \\ \text { I/R } & \text { Ischemia/reperfusion } \\ \text { Lass } & \text { Dehydroceramide synthase } \\ \text { LDH } & \text { Lactate dehydrogenase } \\ \text { LDL } & \text { Low density lipoprotein } \\ \text { LPS } & \text { Lipopolysaccharide } \\ \text { MPO } & \text { Myeloperoxidase } \\ \text { NF } \text { B } & \text { Nuclear factor kappa B } \\ \text { NMDA } & \text { N-Methyl-D-aspartate } \\ \text { NO } & \text { Nitric oxide } \\ \text { NOS } & \text { Nitric oxide synthase } \\ \text { nSMase } & \text { Neutral sphingomyelinase } \\ \text { ORO } & \text { Oil Red O } \\ \text { P } & \text { Postnatal day } \\ \text { PA } & \text { Perinatal asphyxia } \\ \text { PAGE } & \text { Polyacrylamide gel electrophoresis } \\ \text { PAS } & \text { Periodic acid Schiff } \\ \text { PBS } & \text { Phosphate bufferd saline } \\ \text { PC } & \text { Preconditioning } \\ \text { PF } & \text { Paraformaldehyde } \\ \text { PPAR } & \text { Peroxisome proliferator activated receptor } \\ \text { ppia } & \text { Peptidylprolyl isomerase A } \\ \text { qPCR } & \text { Quantitative polymerase chain reaction } \\ & \\ \text { Hel }\end{array}$


RT Reverse transcription

ROS Reactive oxygen species

RXR Retinoid X receptor

SEM Standard error of the mean

SDS Sodium-dodecylsulfate

SMS Sphingomyelin synthase

STAT Signal transducers and activators of transcription

TBS Tris-buffered saline

TBS-T Tris-buffered saline-Triton

TEMED N,N,N',N'-tetramethylethylendiamine

TNF Tumor necrosis factor

TLR Toll-like receptor

TG Triglyceride

TGF Transforming growth factor

VEGF Vascular endothelial growth factor

WB Western Blot 



\section{Acknowledgements/Dankwoord}

Dit proefschrift zou nooit tot stand gekomen zijn zonder de gezamenlijke inspanning, hulp en steun van collega's, vrienden en familie. Met behulp van dit dankwoord wil ik mij bijgevolg richten tot diegenen die mij tijdens mijn promotie-traject begeleid en ondersteund hebben.

Allereerst wil ik beginnen met het bedanken van mijn promotieteam.

Prof. Dr. Vles, mijn promotor; Ik wil u hartelijk bedanken voor de steun en begeleiding tijdens mijn promotieonderzoek. U heeft mij de mogelijkheid gegeven om dit onderzoek te starten waardoor ik mezelf verder kon ontwikkelen, niet alleen op wetenschappelijk maar ook op persoonlijk vlak. We hebben veel gesprekken gehad over het onderzoek maar ook over toekomstplannen; gesprekken waaruit ik veel geleerd heb. Het is ook mede dankzij u dat mijn proefschrift en curriculum vitae zijn zoals opgeschreven in dit boekje. Bedankt voor de fijne samenwerking, uw volhardend vertrouwen, bedankt voor alles!

Prof. Dr. De Baets, Ik wil u bedanken om mij de gelegenheid te geven om dit promotieonderzoek uit te voeren. $U$ had steeds veel vertrouwen in het goede verloop van mijn traject. Bedankt!

Prof. Dr. Kramer, Boris; U bent pas in het laatste stadium van mijn promotie deel geworden van het promotieteam. Toch heeft u gedurende het volledige traject een belangrijke rol gespeeld. Ik ben reeds in mijn Master bij u terecht gekomen om onderzoek te doen naar Chorioamnionitis. Het is tijdens deze periode dat mijn passie voor onderzoek is open gebloeid. $U$ geloofde vanaf het begin dat ik een goede onderzoekster was. U heeft mij voorgesteld aan Danilo en het is dus mede dankzij $\mathrm{u}$ dat ik dit promotieonderzoek heb kunnen doen. Bedankt voor het vertrouwen en alle discussies, bedankt voor alles!

Dr. Gavilanes, Danilo, copromotor; U ligt aan de basis van dit promotieonderzoek. Vanaf dag één van mijn promotie had u reeds zeer veel vertrouwen in mijn kunnen en weten. Steeds weer was u zeer enthousiast over mijn onderzoek. Bedankt voor alle nuttige discussies over data en artikelen. U zorgde ervoor dat ik ook met een klinische blik kon kijken naar mijn "basale" wetenschapsdata. Bedankt om mij de mogelijkheid te geven om dit promotieonderzoek uit te voeren en steeds in mij te blijven geloven, bedankt voor alles! 
To all the co-authors of this thesis: Thank you for your comments and for improving this project.

I would like to thank the members of the assessment committee and corona for their time and critical analysis of my work. I especially want to thank Prof. Dr. Pakkenberg for giving me the opportunity to work in her lab. I sincerely hope that we can continue working together in the future.

Vervolgens wil ik iedereen van Universiteit Maastricht bedanken die mij geholpen heeft tijdens de vijf jaar dat ik in Maastricht was.

Als groentje ben ik begonnen bij de afdeling Moleculaire Genetica. Bedankt allemaal voor jullie hulp en steun tijdens mijn Masterstage en ook nog terwijl ik reeds bezig was aan mijn promotieonderzoek. Ondanks dat ik niet meer bij jullie werkte, kon ik toch nog steeds beroep doen op jullie advies (richt ik me vooral tot Marjon) of gebruik maken van lab-spullen. Veerle, bedankt om mij te begeleiden gedurende mijn Masterstage. Ik heb steeds naar jouw inzet en enthousiasme opgekeken. Veel succes met je verdere onderzoekscarrière! Ronit, Dr. Shiri-Sverdlov, jij leerde me alles wat met onderzoek te maken heeft. Hartelijk bedankt voor alle hulp, advies en inzet die je getoond hebt tijdens mijn Master. Ik waardeer dit enorm en ben bijzonder dankbaar dat ik onder uw begeleiding onderzoek heb kunnen doen.

Verder ook een dankuwel aan iedereen van Kindergeneeskunde. Ook hier kon ik steeds terecht wanneer ik iets nodig had uit het lab. Jennifer en Reint, bedankt voor de gesprekken over van alles en nog wat en veel succes met jullie verdere carrière!

Bedankt aan iedereen van het CPV en de DEC. Tot op het einde van mijn promotieonderzoek stelden jullie steeds alles in het werk om mijn dierexperimenten zo goed en snel mogelijk te laten verlopen. Heel erg bedankt!

Natuurlijk ook hartelijk dank aan iedereen van de afdeling Neuroscience. Ondanks de frustraties die bij een promotieonderzoek komen kijken, heb ik een zeer leuke periode gehad waar jullie een belangrijke bijdrage aan hebben geleverd. De "asfyxie-groep", bedankt voor alle discussies over alles wat je je kan voorstellen. Eveline, bedankt om reeds een goede voorzet te geven in het onderzoek en steeds paraat te staan indien ik ergens hulp bij nodig had. Michelle, zo leuk dat je altijd 
zorgde dat er wat lekkers was, zowel in de kamer maar ook tijdens onze nachtelijke avontuurtjes bij het CPV. Het was altijd gezellig met jou in de beurt. Het analisten team, bedankt dat ik steeds bij jullie terecht kon met al mijn vragen. MarieThérèse en Lisa, steeds even hulpvaardig wanneer ik weer jullie kantoor kwam binnen gewandeld. Rianne en Caroline, het was super met jullie ;-). Tim en Jochen, mijn psychologische begeleiding, bedankt dat ik steeds kon aankloppen wanneer ik het niet meer zag zitten. Chiara, I don't know how I can thank you for all your help and advice! Many, many thanks for everything and all the best and good luck in London! Bedankt aan alle collega's: Alejandro, Jo, Gerard, Kathleen, Sven, Eva B, Fabien, Ali, Ehsan, Marlien, Nienke, Mark, Youssef, Bart, Rinske, Frank, Dagmar, Sarah, Sven, Nick (Groetjes!), Shahed, Kathleen, Annerieke, zonder jullie waren de afgelopen drie jaar niet hetzelfde geweest. Aan alle studenten die hebben bijgedragen aan de publicaties in dit proefschrift; Michelle, Jana, Sara, Annelies and Maria, thank you for your motivation and interest in my research!

To my paranymphs, Maria and Pablo; many thanks that you are willing to stand next to me on my "big day". Maria, I am really proud that I can say that you have been one of my students. You were always available for helping me and even now that you are working on your own PhD-project, you are always dedicated to my project. It was a real pleasure to work with you. Many thanks for all your help and friendship! Pablo, my roommie! I am really grateful that we could share the room together. I think we were the perfect match ;-). I will always remember the nice and 'quiet' moments that we have had! I was really honoured that I could be your paranymph and hope that we will have again a nice time together on my defense. I hope that the three of us can still have many nice moments together and that we can visit each other from time to time!

Ook buiten het werk heb ik steun gehad van zeer veel mensen! De meiden; Eline, Karen en Lize, doordat jullie ook zelf in het onderzoek staan was het altijd fijn om over vanalles van gedachten uit te wisselen. Bedankt om er steeds voor mij te zijn! Iedereen van op ballet en hedendaagse, bij jullie kon ik steeds mijn gedachten verzetten. Hanne, mijn carpool-partner, het zal nu wat moeilijker worden om samen 
te rijden ;-). Bedankt voor alle leuke momenten samen.

En dan is er ook nog een familie en "schoon"familie om trots op te zijn. Robert, Marlies en Maarten; jullie hebben mijn onderzoek steeds met belangstelling opgevolgd. Monique, ondanks je er niet zal kunnen bij zijn, weet ik gewoon dat je vanop een afstandje meekijkt. Bedankt om voor mij een tweede mama te zijn en steeds in mij en mijn onderzoek te geloven.

Mamsie en Papsie, bedankt voor alle steun gedurende mijn hele opleiding. Al vanaf mijn eerste jaar op de universiteit geloofden jullie er in dat ik goed zou worden in wat ik ook maar wilde doen in de toekomst. Jullie zeiden me nooit op te geven en het is dus ook dankzij jullie dat ik mijn promotie tot een goed einde heb kunnen brengen. Jullie toonden steeds interesse in mijn onderzoek en waren soms enthousiaster over mijn artikels en promotie dan ikzelf :-). Ik ben ontzettend blij dat jullie er altijd voor mij zijn. Bedankt voor alles!

Ine, super bedankt voor het ontwerpen van mijn cover! Dit geeft een extra dimensie en emotionele waarde aan mijn boekje. Ik ben er heel erg trots op dat je dit voor mij hebt kunnen doen. Jelle, kleine broertje, nu blijf je nog alleen achter bij mama en papa. Altijd lachen met $\mathrm{u}$ in de buurt! Ben blij dat je er steeds was!

Steven, mijn beertje, jij bent er altijd voor mij. Ik weet dat het niet altijd even gemakkelijk voor je is geweest om al mijn verhalen over het onderzoek te aanhoren. Zelfs nu je in Kopenhagen zit, kan ik nog steeds bij jou terecht met al mijn vragen i.v.m. de lay-out van mijn thesis, het voorbereiden van de promotie, enz... . Bedankt voor al die jaren die we reeds samen hebben beleefd. Hopelijk mogen er nog veel mooie jaren volgen met heel veel gelukkige momenten. Ik heb altijd veel bewondering gehad voor hoe jij tegen het leven aankijkt en ik kijk al uit naar jouw verdediging :-). Bedankt voor alles!!! 


\section{Curriculum vitae}

Evi Vlassaks was born on October 15th 1986 in Genk (Belgium). After graduation in 2004 at the O.L.Vrouw - Lyceum in Genk, she started her Bachelor in Biomedical Sciences at Hasselt University. In 2007, she did the Master Clinical Molecular Life Sciences where she worked on three projects during her Senior internship at the Department Clinical Genetics: Chorioamnionitis induces liver inflammation in fetal sheep; The Alzheimer's disease mouse model (APPSLXPSI) develops hepatic inflammation and steatosis; Deletion of $I \kappa B \alpha$ in bone marrow cells decreases atherosclerotic lesion size, but has no effect on hepatic inflammation. In 2009, she graduated as a Master in Clinical Molecular Life Sciences and started her PhDproject at the School of Mental Health and Neuroscience (MHeNS) at Maastricht University in collaboration with the Department of Pediatrics under supervision of Prof. Dr. JSH Vles, Prof. Dr. MH de Baets, Prof. Dr. BW Kramer and Dr. AWD Gavilanes. During this 3-year PhD-period she investigated the underlying mechanisms of inflammation caused by perinatal asphyxia and chorioamnionitis, as presented in this thesis. During this time, she also mentored several bachelor and master students during their internship, was tutor in some courses and practicals and she attended several national and international conferences where she was able to present her work both orally and by poster presentation. The three last months of her PhD period, she spent abroad at the Research Laboratory for Stereology and Neuroschience at Bispebjerg Hospital in Copenhagen (Denmark) under supervision of Prof. Dr. B Pakkenberg and Dr. T Brudek. Moreover, she recieved the Boehringer Ingelheim Travel grant to cover her research stay.

Evi Vlassaks werd geboren op 15 oktober 1986 in Genk (België). Na het voltooien van haar Middelbare opleiding in 2004 aan het O.L.Vrouw - Lyceum in Genk begon ze haar Bachelor in Biomedische Wetenschappen aan Universiteit Hasselt. In 2007, volgde ze de Master in Klinische Moleculaire Le-venswetenschappen waar ze aan drie projecten werkte tijdens haar Masterstage bij de afdeling Klinische Genetica: Chorioamnionitis induceert lever inflammatie in foetale schapen; Het Alzheimer muizenmodel (APPSLXPSI) ontwikkelt lever inflammatie en steatose; Deletie van $I \kappa B \alpha$ in beenmerg cellen vermindert de omvang van atherosclerotische laesies, 
maar heeft geen effect op lever inflammatie. In 2009 behaalde ze de Master in Klinische Mole-culaire Levenswetenschappen en begon ze als promovenda aan de School of Mental Health and Neuroscience (MHeNS) van Universiteit Maastricht in samenwerking met het departement Kindergeneeskunde onder begeleiding van Prof. Dr. JSH Vles, Prof. Dr. MH de Baets, Prof. Dr. BW Kramer en Dr. AWD Gavilanes. Tijdens dit 3-jarige PhD-traject onderzocht ze de onderliggende mechanismen van inflammatie veroorzaakt door perinatale asfyxie en chorioamnionitis, zoals beschreven in dit proefschrift. Tijdens deze periode was ze ook betrokken in de supervisie van verschillende bachelor en master studenten, was ze tutor in verschillende cursussen en practica en nam ze deel aan verscheidene nationale en internationale congressen waar ze haar werk zowel oraal als via poster presenteerde. De drie laatste maanden van haar $\mathrm{PhD}$ periode verbleef ze in het buitenland aan het Research Instituut voor Stereologie en Neuroscience, Bispebjerg Hospital in Kopenhagen (Denemarken) onder begeleiding van Prof. Dr. B Pakkenberg and Dr. T Brudek. Om haar verblijf te overbruggen, ontving ze de Boehringer Ingelheim Travel grant. 


\section{Peer-reviewed publications}

Vlassaks E, Gavilanes AWD, Bieghs V, Reinartz A, Gassler N, Van Gorp PJ, et al. Antenatal exposure to chorioamnionitis affects lipid metabolism in 7-week-old sheep. Journal of Developmental Origin of Health and Disease, 2012; Volume 3(2), pp 103-110.

Vlassaks E, Bieghs V, Custers A, Van Gorp PJ, Gijbels MJ, Bast A, et al. Chorioamnionitis induced hepatic inflammation and disturbed lipid metabolism in fetal sheep. Pediatric Research, 2010; Volume 68, pp 466-472.

Vlassaks E, Gavilanes AWD, Vles JSH, Deville S, Kramer BW, Strackx E, MartinezMartinez $P$. The effects of fetal and perinatal asphyxia on neuronal cytokine levels and ceramide metabolism in adulthood. In press (Journal of Neuroimmunology).

Vlassaks E, Strackx E, Vles JSH, Martinez-Martinez P, Kramer BW, Gavilanes AWD. Fetal asphyctic preconditioning modulates the acute cytokine response thereby protecting against perinatal asphyxia in neonatal rats. In revision (Journal of Neuroinflammation).

Vlassaks E, Gavilanes AWD, Nikiforou M, Strackx E, Bekers O, Gazzolo D, et al. Acute and chronic immunomodulatory changes in rat liver after fetal asphyctic preconditioning and perinatal asphyxia. Submitted to PlosOne.

Vlassaks E, Mencarelli C, Nikiforou M, Strackx E, Ferraz MJ, Aerts JM et al. Fetal asphyxia induces acute and persisting changes in the ceramide metabolism in the brain. Submitted to Journal of Lipid Research.

Strackx E, Sparnaaij MAR, Vlassaks E, Jellema R, Kuypers E, Vles JSH, et al. Lipopolysaccharide-induced chorioamnionitis causes acute inflammatory changes in the ovine central nervous system. Submitted to Journal of Central Nervous System and Neurological Disorders.

Sparnaaij M, Chedraui P, Strackx E, Vlassaks E, Liem KH, Escobar GS, et al. Fetal asphyctic preconditioning results in preserved placental protein expression of IL-10 at birth. Submitted to Placenta. 


\section{Oral Presentations}

September 2011: EURON and THEME joint PhD meeting (University of Bonn, Germany)

Title: Modulation of fetal asphyctic preconditioning by ceramide signaling

November 2010: GROW Research Day (Maastricht University, the Netherlands)

Title: Effects of asphyctic preconditioning and global birth asphyxia on the neuronal inflammatory response

\section{Poster Presentations}

October 2012: European Academy of Pediatric Societies (Istanbul, Turkey)

Title: Acute and chronic immunomodulatory changes in rat liver after fetal asphyctic preconditioning and perinatal asphyxia

November 2011: GROW Research Day (Maastricht University, the Netherlands)

Title: Mild fetal asphyxia during brain development induces changes in the ceramide pathway

May 2011: Pediatric Academic Societies Annual Meeting (Denver, CO, United States)

Title: Modulation of the acute brain cytokine response contributes to neuroprotection in fetal asphyctic preconditioning

February 2011: MHeNS Research Day (Maastricht University, the Netherlands)

Title: Modulation of the acute brain cytokine response contributes to neuroprotection in fetal asphyctic preconditioning

November 2010: GROW Research Day (Maastricht University, the Netherlands)

Title: Ceramide signaling regulates the effects of fetal asphyxia 
March 2010: MHeNS Research Day (Maastricht University, the Netherlands)

Title: The chronic effects of foetal asphyxia and preconditioning on the neuronal and peripheral immune response

April 2009: Maastricht Medical Students Research Conference (Maastricht University, the Netherlands)

Title: The effects of chorioamnionitis on liver inflammation in prenatal and postnatal sheep

\section{Awards}

Boehringer Ingelheim Fonds Travel Grant (2012) 